\title{
CAEP/ACMU 2014 Scientific Abstracts, May 31 to June 4, 2014, Ottawa, Ontario
}

Research in emergency medicine addresses issues that affect all aspects of patient care, including clinical decision rules, wait times, overcrowding, improved practice guidelines based on best evidence, and new applications for diagnostic tools. Fostering a rich research environment requires education, funding, and a rigorous peer-review process. The Research Committee is pleased to have continued its support of these efforts.

Research-based abstracts are the core of the annual research competition, and this year, there was a record number of submissions for the CAEP 2014 Conference in Ottawa. We received abstracts from emergency medicine researchers from across Canada and internationally. The top ranking abstracts submitted by CAEP members were afforded the opportunity to present at the plenary session, and the best resident, pediatric, and medical student abstracts were awarded financially to subsidize conference travel expenses. The promotion and dissemination of high-quality research, like that submitted to the CAEP 2014 Conference, is integral to the enhancement of Canadian emergency medicine research.

Accompanying these customary research-based education abstracts, the Research Committee accepted education innovation abstracts. These abstracts highlight the novel ideas, methods, and materials developed by emergency medicine educators and focus on curricula, teaching methods, and assessment. The Research Committee has incorporated these innovation abstracts throughout the sessions, as lightning orals, moderated posters, and posters.

As part of the Research Committee commitment to support emergency medicine researchers, five CAEP research grants were awarded for the best proposals submitted by medical students, residents, and junior investigators. These small grants are known to have successfully launched new projects and the careers of new emergency medicine researchers by providing funds at a pivotal point. The funding of these grants requires the support of generous members of our community, so remember to support the Research Committee.

Although not sufficiently recognized, the work of the volunteer reviewers is critically important to the success of the Research Committee activities during the abstract and grant competitions. Each submission is thoroughly read, reviewed, and scored by at least three experienced reviewers. Those submitting grant proposals are supplied with detailed reviewer comments, providing valuable feedback for these junior investigators. The Research Committee would like to thank the reviewers for their hours of work and recognize their commitment to support emergency medicine research.

Finally, CAEP is dedicated to supporting Canadian emergency medicine research and is looking forward to continuing these efforts to encourage new and established researchers.

Disclaimer: The large number of submitted abstracts and the deadlines associated with publication do not permit the author communication, abstract revisions, or CJEM editorial review. The abstracts are presented, as they were submitted to the Research Committee. Only the author affiliation supplied by the presenting author is specified.

Note: The CAEP 2014 Final Program contains the scheduled times for the abstract presentations.

Dr. Rob Green, Chair, CAEP/AMCU Research Committee

\section{CAEP/ACMU Research Awards 2014}

First place, Plenary Presentation, Grant Innes Research Paper and Presentation Award

Paul Atkinson

PL01 Does use of a point of care ultrasound protocol improve outcome in emergency department patients with undifferentiated hypotension? The first Sonography in Hypotension and Cardiac Arrest in the Emergency Department (SHOC-ED 1) Study: an interim analysis.

Second place, Plenary Presentation

Jeffrey Perry

PL02 Prospective validation of the Ottawa Subarachnoid Hemorrhage Rule in headache patients

Third place, Plenary Presentation

Natalie Le Sage
PL03 Criteria for transfer to tertiary trauma centers: validation phase based on the severity of cases treated in tertiary centers

Fourth place, Plenary Presentation, Top Resident Abstract Award Andrei Karpov

PL04 Validation of trigger tools and the National Electronic Injury Surveillance System-Cooperative Adverse Drug Event Surveillance Project (NEISS-CADES) algorithms for identification of adverse drug events within emergency department health records

Top New Investigator Award

Steve Lin

LO26 Targeted temperature management: exploring the association between processes of care and outcomes after out-of-hospital cardiac arrest

Top Medical Student Project Award

Hannah Buhariwalla

LO27 Innovative use of AED by RNs and RTs during in-hospital cardiac arrest 
Top Pediatric Abstract Award

Alexander Sasha Dubrovsky

OP07 Point-of-care ultrasound accurately guides reductions of distal forearm fractures in children

\section{CAEP Resident Research Abstract Awards}

Robert Ohle

LO32 Ultrasonography of the optic nerve sheath diameter for detection of raised intracranial pressure: a systematic review and meta-analysis

Keerat Grewal

LO04 Peripheral vertigo discharged from the emergency department: examining outcomes in the province of Ontario

Justin Yan

LO35 Ketamine-propofol vs propofol alone for procedural sedation in the emergency department: a systematic review and meta-analysis

Joanna Moore

LO28 Was the return of spontaneous circulation protocol effective? Survival to discharge and adherence to the protocol through a health record review

Huma Ali

LO36 Pediatric pain management: the emergency medicine residents' perspectives

Wailliam Lee

LO12 Effect of triage nurse initiated radiography using the Ottawa Ankle Rules on emergency department length of stay at a tertiary care center
Emily Sullivan

OP01 Emergency physicians as human billboards for injury prevention messaging: a randomized controlled trial

\section{CAEP Research Grant Recipients}

Rose Mengual

What is the value of initial blood lactate level in predicting morbidity and mortality in adult patients presenting in the pre-hospital or emergency department setting with presumed infection, sepsis, severe sepsis or septic shock?

Lianne McLean

Point-of-care ultrasound-guided axillary nerve block versus procedural sedation for the reduction of forearm fractures in the pediatric emergency department.

Kaitlin Hayman

Does utilization of an intubation safety checklist improve patient safety during simulated resuscitation scenarios?

Steve Lin

Evaluating cardiopulmonary resuscitation quality measures for the complete duration of resuscitation in out-of-hospital cardiac arrests

Geneviève Tourigny-Ruel

Randomised controlled trial of intravenous dexamethasone to prevent relapse in the treatment of migraine in a pediatric emergency department

Abbreviations: $\mathrm{PL}=$ Plenary; $\mathrm{OP}=$ Oral presentation; $\mathrm{LO}=$ Lightning oral; $\mathrm{MP}=$ Moderated poster; $\mathrm{P}=$ Poster

\section{Oral Presentations}

PL01

Does use of a point of care ultrasound protocol improve outcome in emergency department patients with undifferentiated hypotension? The first Sonography in Hypotension and Cardiac Arrest in the Emergency Department (SHOC-ED 1) Study: an interim analysis

P.R. Atkinson, MD, J. Milne, D. Lewis, MBBS, L. Diegelmann, MD, J. Fraser, BScN, P. Olszynski, MD, M. Stander, MB BCh MMed EM, H. Lamprecht, MBChB, Dalhousie University, St. John, New Brunswick

Introduction: Point of Care Ultrasound (PoCUS) is widely used in emergency department patients to answer specific diagnostic questions. While PoCUS protocols have been shown to improve early diagnosis, there is little published evidence of any mortality benefit in patients with undifferentiated non-traumatic hypotension. We initiated a multicenter randomized controlled trial to assess the impact of the Abdominal and Cardiothoracic Evaluation by Sonography in Shock (ACES) protocol on survival and key clinical outcomes in this group. We report an interim analysis. Methods: Screening at triage identified patients ( $\mathrm{SBP}<100$ or shock index $>1$ ) who were randomized to ultrasound or control group (standard care). An initial and secondary diagnosis was recorded at 0 and 60 minutes. A POCUS-accredited physician performed an ACES scan in the ultrasound group prior to the secondary assessment. Primary outcome measures were 7 and 30-day mortality. Secondary outcome measures included time to normalization of vital signs and acid-base status, and correction of initial diagnoses. Categorical data was analyzed using Fishers two-tailed test, and continuous data by Student $T$ test (GraphPad). Results: 142 patients have been enrolled with follow-up completed. There was no difference in 7-day

mortality; PoCUS 24.6\% (95\% CI 14.1-35.0\%) vs Control 19.6\% (9.6$29.6 \%$; $\mathrm{p}=0.5$ ) or 30 -day mortality; PoCUS $37 \%$ (24.1-49.9\%) vs Control $37.7 \%(24.6-50.8 \% ; \mathrm{p}=1.0)$. PoCUS resulted in a change in initial patient volume status impression in $38.4 \%(27.2-49.5 \%)$ of cases vs only $14.5 \%(5.7-23.2 \%)$ using repeated clinical assessment in the control group $(\mathrm{p}=0.001)$. There were no other significant differences in secondary outcome measures. Diagnostically, PoCUS use was associated with a greater rate of change in working diagnosis than control $(39.2 \%(25.2-46.5 \%)$ vs $16.2 \%(9.1-23.2 \%) ; p=0.0192)$, but without a significant improvement in diagnostic accuracy (PoCUS 68.5\% (56.4$80.6 \%$ ) accurate vs Control 58.0\% (44.3-71.7\%); $\mathrm{p}=0.295)$. Conclusion: An interim analysis of the data in this RCT did not show any mortality or clinical outcome benefit with the use of a PoCUS protocol in undifferentiated hypotensive emergency department patients. There may be some early diagnostic benefits. It is possible that the rate of finding reversible life threatening conditions with PoCUS is too low to impact mortality. We plan to continue to expand recruitment to determine if any true benefit may exist.

Keywords: point-of-care ultrasound, shock, hypotension

PL02

Prospective validation of the Ottawa Subarachnoid Hemorrhage Rule in headache patients

J.J. Perry, MD, MSc, M. Sivilotti, MSc, MD, J. Sutherland, MEd, C.M. Hohl, MDCM, MHSc, M. Emond, MD, MSc, G. Wells, PhD, H. Lesiuk, MD, I.G. Stiell, MD, MSc, Ottawa Hospital Research Institute, Ottawa, ON

Introduction: The decision to investigate acute headache patients for subarachnoid hemorrhage (SAH) is a common dilemma in emergency medicine. A previous study found the Ottawa SAH Rule had retrospective sensitivity of $100 \%$ (95\% CI: 97.2 to $100 \%$ ) with specificity of 
$13.9 \%$ (95\% CI: 12.5 to $15.5 \%$ ). We sought to prospectively validate the Ottawa SAH Rule in a new cohort of patients with acute headache. Methods: We conducted a prospective cohort study in 6 universityaffiliated Canadian emergency departments from 2011 to 2013. We enrolled neurologically intact adults presenting with a new, acute nontraumatic headache peaking within one hour. Physicians explicitly scored the previously derived Ottawa SAH Rule on a standardized study form prior to testing. We conducted telephone and medical record follow-up at 1 and 6 months. SAH was defined as: 1) SAH on $\mathrm{CT}$; 2) visual xanthochromia in the cerebrospinal fluid (CSF); or 3) red blood cells in the final tube of CSF with positive cerebral angiography. We calculated sensitivity and specificity with corresponding $95 \%$ confidence intervals. Results: We enrolled 1,140 (73.2\% of eligible patients), including $64(5.6 \%)$ with SAH. Subjects were of mean age 43.9 years (SD 16.5), female $59.6 \%$, median headache severity of 10/10 (IQR 8-10), and 87.1\% underwent CT. The Ottawa SAH Rule had a sensitivity of $100 \%$ (95\%CI: 94.3 to $100 \%)$ and specificity 14.3\% (95\%CI: 11.7 to $15.6 \%$ ) for SAH. Conclusion: The Ottawa SAH Rule is a valid, highly sensitive instrument for identifying SAH among neurologically intact patients presenting to the emergency department with acute headache. The Ottawa SAH Rule will help standardize which patients require investigations.

Keywords: subarachnoid hemorrhage, clinical decision rule

\section{PL03}

Criteria for transfer to tertiary trauma centers: validation phase based on the severity of cases treated in tertiary centers

N. Le Sage, MD, MSc, A. Lavoie, PhD, L. Moore, PhD, R. Verreault, $\mathrm{PhD}, \mathrm{M}$. Emond, MD, MSc, Université Laval, Québec, QC

Introduction: Recently, a consensus on the indications for transfer to a tertiary trauma center was obtained from 83 experts representing four disciplines (emergency medicine, surgery, neurosurgery and intensive care) who are involved within the integrated trauma system. This study aims to statistically validate these indications for patient transfer. Methods: Each criteria was converted into a consensus indicator in order to enable a statistical validation using a trauma registry. The study population consisted of all patients in the registry who were directly transported to a tertiary care center between 1998 and 2008. We compared the rate of intrahospital death, admission to intensive care unit and intrahospital complications in patients responding to either of the criteria over those not meeting the criteria. Results: Data from 27480 major trauma patients were analyzed. The risk of death, ICU admission and severe complications [CI 95\%] of patients responding to either of the criteria of consensus is higher than that of patients with no criteria (respectively RR $=6.1$ [5.5-6.7], 3.9 [3.8-4.1] and 2.8 [2.6-2.9]). Each individual criteria significantly increases the risk of death $(\mathrm{p}<0.001)$ with the exception of spinal injury, an entity that is most often a single trauma not putting the patient's life in danger. Conclusion: These results suggest that the criteria are valid injury severity indicators and that these patients require a higher level of care. However, they also need to be validated on the ability of transfer to improve the prognosis of these patients.

Keywords: transfer, trauma care organization, major trauma

\section{PL04}

Validation of trigger tools and the National Electronic Injury Surveillance System-Cooperative Adverse Drug Event Surveillance Project (NEISS-CADES) algorithms for identification of adverse drug events within emergency department health records

A. Karpov, MD, C. Mok, MSc, C. Parcero, C. Panditha, BTech, L. Dempster, BScN, E. Yu, MSc., C.M. Hohl, MDCM, MHSc, Department of Emergency Medicine, University of British Columbia
Introduction: Adverse drug events (ADEs), harmful and unintended events related to medication use, are a common cause of emergency department (ED) visits. Tracking ADEs within ED data is important for drug safety and effectiveness research, post marketing surveillance, and quality improvement efforts to reduce ADEs. Trigger tools (TT) and the National Electronic Injury Surveillance System-Cooperative Adverse Drug Event Surveillance Project (NEISS-CADES) algorithms are explicit chart review methods recommended by patient safety organizations to identify and track ADEs within medical records. Our objective was to evaluate their sensitivity and specificity in identifying ADEs in ED records. We hypothesized that their sensitivity in identifying ADEs would be low. Methods: This was a planned sub-study of a prospective study conducted in one Canadian tertiary care ED with a census of 83,000 patients. We enrolled patients between July 2008 and January 2009. Clinical pharmacists approached incoming patients using a systematic selection algorithm and screened them for eligibility. Pharmacists prospectively evaluated enrolled patients for ADEs using a standardized process. We then interviewed treating physicians who were blinded to the pharmacists' diagnosis to identify alternate diagnoses. For cases in which the pharmacists' and physicians' diagnoses were concordant (ADE/ADE; no ADE/no ADE), this was considered the criterion standard. An independent committee adjudicated all other cases. Three research assistants, blinded to all research records, reviewed the medical charts of enrolled patients and applied the TT and NEISS-CADES algorithms to identify ADEs. We compared ADE cases identified using the TT and NEISS-CADES methodologies with those identified prospectively, and calculated the sensitivity and specificity with $95 \%$ confidence intervals (CIs) of these detection methods. The sample size was a function of the primary study. Results: We enrolled 1,151 patients in the study. Among included patients, the average age was 51.6 and $53 \%$ were female. We identified 164 ADEs among 152 patients. The sensitivity of seven TTs was $2.6 \%(95 \%$ CI $0.7-6.6 \%)$ to $12.5 \%(95 \%$ CI $7.9-19.1 \%)$ for patients with $>1$ ADE. All tools had a specificity of at least $99.3 \%(95 \% \mathrm{CI}$ 98.6-99.7). The NEISS-CADES algorithm was $15.8 \%$ (95\%CI 10.6$22.8 \%$ ) sensitive, and $99.5 \%$ (95\%CI 98.8-99.8\%) specific. Conclusion: The sensitivity of the TT and NEISS-CADES methods for ADEs is low. More robust methods need to be developed to identify ADEs within ED records.

Keywords: ADE, trigger tool, NEISS-CADES

OP01

Emergency physicians as human billboards for injury prevention messaging: a randomized controlled trial

E. Sullivan, MD, Q.S. Paterson, R. Woods, MD, MMEd, D. Fuller, $\mathrm{PhD}$, University of Saskatchewan, Saskatoon, SK

Introduction: Preventable injuries are a common cause of morbidity and mortality encountered in emergency departments (EDs). Emergency physicians (EPs) focus their efforts on diagnosis and treatment, while counseling on health promotion and injury prevention does not often occur. This randomized controlled trial (RCT) examined the impact of a novel health promotion tactic involving EPs wearing scrub tops with a bicycle helmet-promoting message. Methods: This was an RCT done in two tertiary EDs and a pediatric ED in Saskatoon, Canada. Fourteen EPs were observed for two shifts each between June and August of 2013. Each pair of shifts was randomized to either an injury prevention shift, during which the EP would wear the customized scrub top, or a control shift. The primary outcomes were the number of seconds spent on injury prevention counseling and a dichotomous indicator of any counseling. The secondary outcome was a change in selfreported helmet use at one-month follow-up. Results: There were a total of 244 patient encounters (134 control, 110 intervention). Of the patient encounters, 33 (control) and 18 (intervention) received injury 
prevention counseling. The average time spent on injury prevention discussion was 3.36 seconds. There was no difference in the likelihood of injury prevention discussion between the treatment $(\mathrm{OR}=0.59,95 \% \mathrm{CI}$ : 0.30 to 1.16 ) and control group. 133 individuals cycled and 20 reported always wearing a helmet prior to this visit. $37 \%$ of patients who always wore a helmet reported that the visit had reinforced their decision to wear a helmet and $25 \%$ of patients who often, seldom, or never wore helmets reported that the visit had changed their decision to wear a helmet. However, at one month follow-up there was no significant difference in reinforcement or change in helmet-wearing between the control and treatment groups. Conclusion: Injury prevention messaging on scrub tops worn by EPs did not increase the amount of physicianpatient discussion on injury prevention. Given that our sample size was relatively small, this novel health promotion study should be replicated with more providers involved (allied health, residents, nurses). This would allow for a more complete examination of this simple intervention's potential for health behavior change in patients.

Keywords: injury prevention, randomized controlled trial, emergency medicine

\section{OP02}

Portrait of rural emergency departments in Québec and utilisation of the Québec Emergency Department Management Guide: Phase 1

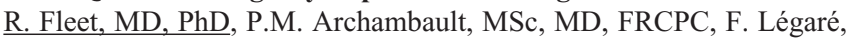
, MD, PhD, CCFP, J. Chauny, MD, MSc, L. Jean-Frédéric, MD, M. Ouimet, PhD, G. Dupuis, PhD, J. Haggerty, PhD, A.B. Tanguay, MD, MSc, G. Simard-Racine, MD, J. Gauthier, MSc, J. Poitras, MD, Université Laval, CHAU Hôtel Lieu de Lévis, QC

Introduction: Rural emergency departments (EDs) are important safety nets for the $20 \%$ of Canadians who reside there. Surprisingly, information on these EDs is scarce. Pilot data suggests important interprovincial differences in access to services in rural EDs. Québec appears to provide more comprehensive access that may be attributed to policy/guidelines (Québec ED management guide (QEDMG)). This hypothesis requires further study. Methods: We selected EDs offering 24/7 medical coverage, having hospitalization beds and located in "rural or small towns". We collected data using phone, paper and online surveys with rural ED/hospital staff. Data was also collected from Québec Ministry of health databases and statistics Canada. We computed descriptive statistics. Results: Among Québec's 26 EDs, 23 consented to participate $(88.5 \%)$. These EDs are located in communities with a median population of 5940 (IQR: 2401 - 7914). The median annual ED census was 19594 (Interquartile range (IQR): 14971 21433). The proportion of patient- visits according to triage level was $0.5 \%, 2.2 \%, 19.6 \%, 35.6 \%$ and $42.1 \%$ for level 1 to 5 respectively. Most commonly, ED physicians were recent graduates with less than 5 years of experience $(31 \%)$. Seven percent had residency training/certification in emergency medicine Approximately, $40 \%$ of shifts were covered by "full time" ED physicians, 14\% by locums. Typically, shifts are covered by solo physicians teamed with 2 to 3 nurses. Access to the following 24/7 services was: X Ray/lab 100\%, CT scanner $74 \%$; ICU 78\%; psychiatrist 48\%; Obstetrician/gynecologist 35\%; Surgeon $78 \%$; pediatrician $13 \%$; orthopedist $17 \%$; and anesthetist $65 \%$. Forty two percent of EDs are more than $300 \mathrm{~km}$ away from a level 1 trauma center and 58\% from a level 2 trauma center, and only $33 \%$ of these EDs had air transport access. Roughly, 290 (SD 88) inter-facility transfers were required per year per ED for higher level of care. Approximately $40 \%$ of participants reported having no or little knowledge of the QEDMG and 77\% reported never or only sometimes using the QEDMG. Conclusion: Québec rural EDs are staffed by relatively new graduates working as solo physicians in well-resourced, moderately busy EDs (by rural standards). These EDs are distant from trauma cen- ters and have limited access to air transport. QEDMG may have contributed to this model of service attribution favoring more local services versus inter-facility transport but, for the most part, staff report limited knowledge or use of Québec Guidelines.

Keywords: emergency department, rural, access

OP03

Relationship between mental health apprehensions by police and monthly income assistance (welfare) payments

T.A. Pickett, MD, R. Stenstrom, MD, PhD, R.B. Abu-Laban, MD, MHSc, St. Paul's Hospital, Vancouver, BC

Introduction: Social misconduct, increased police activity, and increased Emergency Department (ED) utilization have all been associated with monthly lump-sum income assistance (welfare) payments in both Canada and the United States, however the relationship between drug induced or organic psychiatric illness and the release of income assistance payments is poorly understood. The purpose of this study was to determine if a relationship exists between the number of Mental Health Apprehensions (MHAs) by police and monthly welfare cheque distribution. We hypothesized that there would be more MHAs in the days on or immediately following cheque distribution. Methods: Data from the administrative database of the Vancouver Police was explored over an 81week period (8 June 2011 to 25 Dec. 2012). Comparisons were made between the daily numbers of mental health apprehensions by police during the week following income assessment payments to those during non-payment weeks. The comparison groups were established a priori, and their cutoffs were explicitly defined. Based on approximately a 3:1 ratio of non-welfare payment weeks to welfare-payment weeks, we determined 510 days ( 74 weeks) would have $90 \%$ power to detect a 1 apprehension per day mean difference, an amount we deemed to be clinically significant. MHAs were analyzed continuously, and compared using the two-tailed t-test for independent means. Research ethics board approval was obtained. Results: A total of 4009 MHAs occurred during the 567 day study period. The mean number of MHAs per day during welfare disbursement weeks was 7.81 (95\%CI 7.34-8.29), compared to 6.85 (95\%CI 6.61-7.11) during non-payment weeks $(\mathrm{p}=0.003)$. Conclusion: There is a statistically and clinically significant increase in the number of MHAs by police during the week following income assistance payments. This predictable pattern in MHAs should be considered in Emergency Department Psychiatry service coverage, police distribution and staffing models, and community resource planning. Moreover, changing the current British Columbia model of income assistance payment release (for example to a weekly system) could have a smoothing or reducing effect on MHAs over the course of each month, and this possibility merits policy consideration and future investigation.

Keywords: mental health apprehension, police, welfare

\section{OP04}

Little time spent giving discharge instructions in Canadian emergency departments

Q.S. Paterson, E. Sullivan, MD, R. Woods, MD MMEd, D. Fuller, $\mathrm{PhD}$, University of Saskatchewan, Saskatoon, SK

Introduction: Discharge instructions (DI) are crucial for improving patient self-management and safety. Patients typically have poor understanding of their DI, which may lead to poor health outcomes, high return rates, and poor compliance. Best practice guidelines for emergency department DI were recently published, yet no recommendations were made regarding how much time should be spent giving DI. To our knowledge, only three studies have measured time spent on DI. The length of DI varied from 76 seconds to 6 minutes. This study con- 
tributes to the literature by examining the time spent giving DI in two Canadian emergency departments. Methods: This direct observational analytic study took place in two Canadian emergency departments, including one adult and one combined adult/pediatric department. Fourteen volunteer physicians were directly observed by a medical student for two shifts between June and August of 2013. All physicians were blinded to the outcome being measured, the length of time spent giving DI. The student used a stopwatch to record the time the physicians spent giving DI to each patient. Patients $(n=244)$ provided informed verbal consent to the medical student after discharge, provided they met the inclusion criteria (stable vitals, no apparent distress, competent mental status). Descriptive analyses were conducted in Stata (version 12) to examine both the mean time spent giving DI to patients and physician variation in DI time. Results: The mean time spent giving DI by physicians was $\mathrm{M}=71$ seconds $(\mathrm{SD}=77 \mathrm{~s})$. The difference in $\mathrm{DI}$ time for adult patients ( $n=184 ; M=69 \mathrm{~s} ; \mathrm{SD}=74 \mathrm{~s})$ and pediatric patients $(\mathrm{n}=60 ; \mathrm{M}=78$ $\mathrm{s} ; \mathrm{SD}=83 \mathrm{~s}$ ) was not statistically significant. The means between the 14 physicians ranged from 28 to 124 seconds. Conclusion: Consistent with past research, our results show that emergency department physicians spend little time giving DI to patients. Our data shows that Canadian emergency physicians may spend less time on DI compared to American and Swiss physicians; however, with the paucity of research in this area it is difficult to reach a conclusion. Though different methods have been studied in hopes of improving patient comprehension of DI, future research should focus on the implications of spending more time giving DI, regardless of the method.

Keywords: discharge instructions, emergency medicine, physicianpatient interaction

\section{OP05}

An electronic system reduces time to disposition in emergency department patients with chest pain

M. Feldman, MD PhD, B. Demendonca, D. Hefferon, J. Wang, MASc, J. Tyberg, MD, University of Toronto, Toronto, ON

Introduction: Reducing waiting times in the emergency department (ED) is a major thrust of hospital quality improvement initiatives and considerable resources are being devoted to improving this nearly-universal problem. One component of waiting time is the elapsed time between reporting of clinical laboratory investigations and providing an appropriate disposition for the patient, such as referral, admission, or discharge. We recently implemented an electronic 'Push-Alert' notification system that delivers laboratory investigations determined by our hospital lab to be critically abnormal and delivers all cardiac troponin assay results directly to a secure handheld device carried by the emergency physician responsible for that patient. Our objective was to determine the impact of the Push-Alert notification system on the time interval from completion of laboratory study to disposition for ED patients presenting with chest pain or an acute coronary syndrome (ACS). Methods: We conducted a before-after observational study of a convenience sample of all patients presenting to our ED between July 2009 to June 2010 and July 2011 to June 2012 with chest pain or ACS who had two troponins measured. Elapsed time from the second troponin result being made available on the electronic lab reporting system to patient disposition was compared between patients arriving before and after implementation of the Push-Alert system. We excluded records where the test result was an error (such as insufficient quantity or hemolyzed samples). Results: During the study period, a total of 103,043 patients were seen at our ED, of which 47,394 were seen before and 55,649 after the implementation of the Push-Alert system. A total of 22,023 patients presented with chest pain syndrome or ACS, of which 10,944 were seen before implementation and 11,079 after implementation. After implementation, 3,257 patients had alerts sent to their physicians via the Push-Alert system. A total of 792 patients met the inclusion criteria, of which 529 were seen before and 263 after implementation. Median time from completion of the second troponin to disposition was significantly reduced by 46 minutes, from 2.55 hours before implementation to 1.78 hours after, $\mathrm{p}<0.01$. Conclusion: An electronic Push-Alert notification system which automatically delivers troponin values to the patients' emergency department physician significantly reduces the time to disposition of chest pain patients in the ED.

Keywords: medical informatics, acute coronary syndrome

OP06

A determination of pre-triage wait times at two busy acute care EDs and a comparison to CTAS recommendations

S. Trivedi, BSc, M. Betz, MD, J. Stempien, BSc, MD, University of Saskatchewan, Saskatoon, SK

Introduction: Measuring the lengths of stay of patients in an emergency department (ED) is usually documented from the time of registration and triage. The time that patients wait in line prior to registration and triage has not been well documented. We sought to characterize pre-triage wait times and compare them to CTAS time-tophysician recommendations. Methods: This observational study documented the time that consenting patients entered the ED and the time they were formally registered and triaged. Participants' CTAS score were collected from the electronic record. Patients arriving to the ED by ambulance were excluded. Results: A total of 536 participants were timed. Of these, eleven $(2 \%)$ participants left without being triaged. Participants who scored either CTAS 1 or $2(n=53)$ waited a median time of 3.1 (range: 0.05-44.2) minutes. Patients triaged as CTAS 3 $(\mathrm{n}=187)$ waited a median of 11.4 (range: 0.05-91.1) minutes, CTAS 4 $(\mathrm{n}=139)$ a median of 16.6 (range: 0.06-98.6) minutes and CTAS 5 $(\mathrm{n}=146)$ a median of 17.5 (range: 0.05-90.4) minutes. Across all groups, 11 CTAS 2, 34 CTAS 3 and 10 CTAS 4 patients $(\mathrm{n}=55$, $10.5 \%$ ) had exceeded their CTAS time recommendations before being triaged. Conclusion: All urban EDs closely follow patients' wait times, often stratified according to triage category, and are assumed to begin upon patients' arrival in the ED. The study's findings suggest that these times, if included, may take up a portion of or exceed the CTAS time-to-physician recommendations. For some patients this could be clinically significant and as a result EDs should consider documenting time-to-physician times from the moment of patient arrival. Keywords: emergency overcrowding, triage, quality improvement

OP07

Point-of-care ultrasound accurately guides reductions of distal forearm fractures in children

A. Dubrovsky, MDCM, MSc, A. Kempinska, MD, I. Bank, MDCM

Montréal Children's Hospital, Montréal, QC

Introduction: Reduction of forearm fractures in children is frequently performed with the aid of fluoroscopy. The goal of this study is to determine how point-of-care ultrasound compares to fluoroscopy in assessing the adequacy of reduction of single-bone distal forearm fractures in pediatrics. Methods: We completed a prospective observational study in a tertiary-care pediatric emergency department. Eligible patients were $<18$ years of age with acute $(<72$ hours) single-bone fractures of the mid or distal forearm that required reduction. Exclusion criteria included fractures with displacement of the radius and ulna, intra-articular fractures, open fractures or any neurovascular compromise. A one-hour simulation-based session was given to novice-ultrasound emergency physicians who then performed the reduction under ultrasound guidance (high frequency linear probe) until they felt they had the best possible reduction and saved images in at least twoplanes. Based on the saved images, the physician recorded whether the reduction was adequate or if further reduction was necessary. Fluo- 
roscopy was then performed and the physician assessed the fluoroscopic images to decide whether the fracture had been adequately reduced; management continued based on the fluoroscopic images. Results were defined dichotomously as "adequately" or "inadequately" reduced for all images. Results: To date, 97 patients were enrolled. The mean age was $10.8 \pm 3.3$ years and $74.2 \%$ were male. The radius was fractured in $99 \%$ of cases: distal radius $88.7 \%$ of which $26.8 \%$ involved the growth plate. Physicians performed a mean number of 6 (range 1-29) reductions with the aid of ultrasound and ranked its ease of use at a mean of $1.9 \pm 0.9$ on a 5 -point liker scale (1 extremely easy, 5 extremely difficult); $77.3 \%$ reported that ultrasound helped with the reductions. Ultrasound revealed 83 adequate, 11 inadequate, 2 false negative (i.e. ultrasound inadequate but fluoroscopy adequate) and no false positives yielding a sensitivity and specificity $(95 \% \mathrm{CI})$ of $97.7 \%$ $(94.5,100.9)$ and $100 \%$ for adequacy of reduction, respectively. The overall diagnostic accuracy was of $97.9 \%$; only $1 / 16$ physician had discordant fluoroscopy and ultrasound interpretations that occurred during their first cases. Conclusion: Point-of-care ultrasound can be used reliably by most physicians to guide and determine adequacy of fracture reductions of distal radius fractures in children. Optimal training strategies need to be determined in order to ensure accurate interpretations. Keywords: ultrasound, fracture, pediatric

\section{OP08}

Emergency department analgesia in acute appendicitis: a comparison of adult and paediatric patients

C. Krause, MD, E. Karreman, PhD, S.M. Smith, MD, Regina Qu'Appelle Health Region, Regina, SK

Introduction: The under-treatment of pain, known as "oligoanalgesia", is a well-recognized phenomenon in the Emergency Department (ED), and it has been suggested that children are especially at risk. However, there is a paucity of research in this area, especially from general (i.e., mixed adult and paediatric) emergency departments. This study was designed to compare the pain medication received by adult and paediatric patients with appendicitis presenting to a general ED. Methods: A retrospective chart review of 165 consecutive patients, 92 adult (mean age: $35.7 \pm 15.7$ years) and 73 paediatric (mean age: 11.0 \pm 3.0 years) who presented via the ED with a discharge diagnosis of "appendicitis" were included in this study. Demographic information as well as data regarding type, timing, and amount of pain medication given were collected using a standardized data abstraction table. Adult and paediatric data were compared using t-test or chi-square analysis. Effect sizes were also calculated. Results: Mean pain scores recorded at presentation were significantly lower for children vs. adults (6.49 vs 7.19 out of $10, p=0.015, r=.20$ ). Paediatric patients were significantly more likely than adult patients not to receive any analgesia during their ED stay $(58.9 \%$ vs $20.7 \%, \mathrm{p}>0.001$, Cramer's V $=.39)$. They were also significantly less likely to receive opioid analgesics, compared to adults $(27.4 \%$ vs. $71.7 \%, \mathrm{p}>0.001$, Cramer's V $=.44)$. Conclusion: In this sample, paediatric patients with appendicitis presenting to a general emergency department received less opioid pain medication, and less pain medication in general, than their adult counterparts. This could suggest children are receiving inadequate pain control in the ED. Further research is needed to assess whether children's analgesic needs are actually being met and to identify barriers to providing appropriate pain medication in this population.

Keywords: paediatrics, appendicitis, analgesia

\section{OP09}

A randomized controlled trial comparing ultrasound, VeinViewer and standard approach to peripheral intravenous catheter placement in the pediatric emergency department
S.J. Curtis, MD, MSc, W. Craig, MD, MSc, E. Logue, B. Vandermeer, MSc, A. Hanson, MD, T. Klassen, MD, MSc, Women and Children's Health Research Institute, Edmonton, AB

Introduction: Peripheral IV (PIV) line placement is painful, stressful and is the most common procedure performed in the pediatric emergency department (PED). First attempt cannulation success rates are variable and practitioner dependent. Methods: Prospective, randomized controlled trial, stratified by age, comparing three PIV placement approaches: (1) ultrasound (2) VeinViewer (3) standard traditional approach. All children aged 0-16, requiring PIV for routine care in the PED, were approached for enrollment. The primary objective of this study was to investigate whether the use of either ultrasound or VeinViewer could improve the rate of success of the initial attempt at PIV placement in comparison to the current standard approach. Secondary objectives were to investigate, when compared to standard approach, whether the use of ultrasound or VeinViewer (1) reduced the number of IV punctures required to achieve successful PIV placement, (2) reduced the time taken to achieve successful PIV placement, (3) improved nursing satisfaction with PIV placement in children. Results: Four hundred patients were enrolled. Overall success of IV placement on first attempt was similar across study arms: Standard [109/146 (74.7\%)]; Ultrasound [97/137 (70.8\%)]; VeinViewer [89/135 $(65.9 \%)]$; p 0.28 . When stratified, differences were also non-significant. For age 3 and under: Standard [31/50 (62.0\%)]; Ultrasound [22/42 (52.4\%)]; Vein Viewer: [18/43 (41.9\%)]; p 0.15. For age 4 and above: Standard [78/96 (81.3\%)]; Ultrasound [75/95 (79.0\%)]; Vein Viewer [71/92 (77.2\%)], p 0.77. Overall mean number of attempts did not differ significantly between groups: Standard [1.43]; Ultrasound [1.40]; VeinViewer [1.58]. Overall time variables across groups were not significantly different. Mean times (minutes) from verbalization of study arm to successful PIV were: Standard [14.8]; Ultrasound [17.6], VeinViewer [16.1], p 0.44. Mean times (minutes) from tourniquet to time of successful PIV were: Standard [6.5]; Ultrasound [8.3]; VeinViewer [7.8], p 0.50. Nurse satisfaction was not improved by use of either technology. Conclusion: We found no evidence that either Ultrasound or VeinViewer improves the first-attempt success rate, number of cannulation attempts, speed of cannulation for children or nursing satisfaction in the pediatric emergency department.

Keywords: pediatric, randomized controlled trial, technology

\section{OP10}

ASSIST pilot study: accuracy of Inovise STEMI interpretation software

S. Henley, BSc(Hons), D. Munkley, MD, D. Garniss, MD, I. Laidley, MD, M. Welsford, MD, University of Aberdeen, Aberdeen, Scotland

Introduction: Paramedics commonly acquire 12-lead electrocardiograms (ECGs) during first medical contact in patients presenting with symptoms of cardiac ischemia in order to recognize ST-segment elevation myocardial infarction (STEMI). STEMI recognition can be accomplished by a physician via ECG transmission, paramedics directly, or using computerized interpretation. The accuracy of one such new computerized algorithm, Inovise, has neither been evaluated in peer review trials, nor been evaluated in the prehospital environment. The objective of this study is to assess the accuracy of the Inovise 12L Interpretative Algorithm software in the prehospital environment. Methods: This pilot study focuses on ECGs acquired by 2 paramedic services in Ontario, Canada, using the Zoll X portable ECG monitors with the Inovise software. Two hundred ECGs were interpreted by 2 Emergency Medicine physicians using the Third Universal Definition of Myocardial Infarction and compared with the Inovise software. The physicians were blinded to the interpretations and to the 
patients. A third physician was included in cases of disagreement. Anonymised patient information was abstracted from the ambulance call reports using careful retrospective medical review methodology into a database for analysis. Accuracy, sensitivity (Sn), specificity $(\mathrm{Sp})$, positive predictive value (PPV) and negative predictive values (NPV) were calculated. Cohen's kappa was used to assess inter-rater agreement. Results: Prevalence of STEMI in our pilot study was $6.5 \%$. Cohen's kappa for the physician interpretation was 0.55 , which represents moderate agreement. Accuracy of the Inovise software was calculated at $96 \%$. Sp was $99 \%$ (CI 98-100\%), with a low false positive rate for inappropriate STEMI alert activation. However, sensitivity was $45 \%$ (CI 37- 53\%) missing over half of the STEMIs identified by the Emergency Physicians. The PPV was $83 \%$ (CI 77- 89) and the NPV was $96 \%$ (CI 93-99\%). There is significant difference $(\mathrm{p}<0.001)$ between the results of this pilot study in actual prehospital use and those of the manufacturer's white paper (Sn 77\%, Sp 99\%, PPV 97\%). Conclusion: This pilot study is underpowered and thus will require a larger study to confirm. However, if these results were subsequently confirmed in the larger trial, computerised ECG interpretation software with such a low sensitivity and PPV should not be used as the sole method for STEMI screening by paramedics.

Keywords: emergency medical services, STEMI, ECG interpretation

OP11

Description of a successful longitudinal pre-hospital care emergency medicine curriculum

T.M. Chan, MD, A. Pardhan, MD, M. Welsford, BSc, MD, McMaster University, Hamilton, ON

Innovation concept: Education in pre-hospital care (PHC) and Emergency Medical Services (EMS) is an important part of all Canadian Emergency Medicine residencies. Understanding how the complex EMS system works is important to ensuring seamless and efficient care within the greater healthcare system and sets the groundwork for residents interested in pursuing fellowship opportunities in PHC. Traditional one-month blocks, however, have provided inadequate exposure to the full breadth and complexity of the EMS system, providing only a superficial understanding of PHC and medical direction. Methods: In the fall of 2009, we launched a longitudinal PHC core curriculum for PGY2s in the Royal College Emergency Medicine (EM) Residency Program at McMaster University, which was based on a blended competency and experiential framework. Over the course of 9 months during their EM rotations, our PGY2 residents are given a framework to complete specific objectives and goals that includes didactic education, self-study, completing experiential tasks (e.g. ride outs, participating in paramedic teaching sessions), as well as completion of quality assurance, quality improvement (QI), and optional research projects. The competency-based assessment for this program consists of a portfolio, requiring a mix of assessments including: 1) Reflection and proof of experience completion (e.g. managing EMS patch calls, ambulance ride-outs); 2) Submission of the quality assurance project; 3) Submission and presentation of the QI or research project; 4) Oral evaluation (cognitive simulation around prehospital decision making); 5) Written evaluation (format similar to final Royal College written evaluations); 6) Exit interview to ensure all objectives and goals are met.

Curriculum, tool, or material: We have seen extensively increased scholarly work in the area of prehospital care (PHC) (e.g. presentations at Research Day included 0 or 1 projects annually in PHC in 20062009 , to 5 to 11 projects in 2010-2013). Since the program's inception, many of the residents $(\sim 33 \%)$ who completed a PHC project as a junior, also continued to abstract and manuscript submission. Conclusion: A longitudinal PHC rotation with competency-based assessments is possible and increases the scholarly output of the residency program.
This unique format may be expanded to include both training streams and to other Emergency Medicine residency programs across Canada. Keywords: innovations in EM education, residency education, prehospital care

\section{OP12}

The use of medic alert notification by paramedics and medical communication officers

A. Carter, MD, M. Bohrer, BSc, J.L. Jensen, BSc, EHS, Halifax, NS

Introduction: Medic Alert (MA) EnRoute Notification (MAN) allows paramedics and medical communication officers (CO) to access the MA record. This study seeks to understand barriers and facilitators to the uptake of MAN. Technology is increasingly being used to support health care, and understanding the challenges and opportunities will be an important step to successful implementation. Methods: This Theory of Planned Behaviour (TPB) study explores paramedic and CO intent to use MAN. TPB examines intent through three constructs: behavioural beliefs (BB - attitude), subjective norms ( $\mathrm{SN}$ - social pressure) and perceived behavioural control (PBC - control to do the behaviour). Semi-structured interviews (previously reported) were used to develop a paper-based survey, which was piloted and distributed. Intent was assessed along with direct and indirect measures for each construct on a 5-point Likert scale. Data were analyzed in SAS using regression analysis, following the TPB model. Results: 67 paramedics participated; mean age 36 years (SD 9.7), mean years' experience 11 (9.9), had used MAN mean 3.1(5.0) times. Paramedics intention to use MAN if it works well was 4.0 (median). BB was most associated with intention, median score 4 (IQR 3.5-4.5); SN 3.0 (2.5-3.5); PBC 4.0 (3-5). Indirect statements that were significantly associated with intention described accessing MAN en route to calls (OR 0.84 (95\%CI $0.76-$ $0.95)$ ) and increased awareness of MAN (OR 0.79 (95\%CI 0.65-0.97)). 24 COs participated; age 35 years (SD 6.3), years' experience 6 (6.6) and used MAN 6(13.3) times. CO intention to use MAN scored median 2.9. BB was the most important construct: median 4.0 (IQR 3.0-4.5); SN 3.0 (2.0-3.0); PBC 2.8 (2.4-3.2). Indirect measures significantly associated with intention were about COs knowing paramedics used the information COs retrieved OR 0.74 (95\%CI 0.56-0.97), if callers had their MA number OR 0.67 (95\% CI $0.45-0.99)$ and increased awareness about MAN OR 0.82 (95\% CI 0.69-0.98). Conclusion: Attitudes towards MAN are strongly associated with intention to use it. Ease of use emerged as an important barrier in Phase I. Paramedics intend to use MAN, particularly if they could access it en route to calls. COs have lower intent to use MAN, noting poor return on effort of questioning. Both groups identified need for more awareness on MAN. These provide important targets for increased uptake of new health technologies.

Keywords: emergency medical services, health information technology

OP13

Systematic review and meta-analysis of the benefits of prehospital 12-lead ECG confirmation in STEMI patients

J. Nam, MSc, K. Caners, BSc, MD, J. Bowen, BScPhm, MSc, M. Welsford, BSc, MD, D. O'Reilly, MSc, PhD, Division of Emergency Medicine, Department of Medicine, Faculty of Health Sciences, McMaster University, Hamilton, ON

Introduction: Prehospital identification of ST-segment elevation myocardial infarction (STEMI) patients transported by emergency medical services (EMS) with 12-lead electrocardiography (ECG) confirmation can improve patient outcomes. Previous reviews of this strategy showed imprecision and were published prior to the release of a number of newer studies. The objective of this study was to present an 
updated review of prehospital identification of STEMI patients transported by EMS with 12-lead ECG confirmation versus standard or no cardiac monitoring. Methods: EMBASE, PubMed, and Cochrane Library were searched using controlled vocabulary and keywords. Randomized controlled trials and observational studies were included. Outcomes included short-term mortality ( $\leq 30$ days), door-toballoon/needle time or first medical contact-to-balloon/needle time. Pooled estimates were determined, where appropriate. Results were stratified by percutaneous coronary intervention (PCI) or fibrinolysis. Results: The search yielded 1,857 citations of which 68 full-texts were reviewed and 16 studies met the final criteria: 15 included data on PCI and 3 on fibrinolysis ( 2 included both). Observational studies limited the quality of evidence; however, a number of studies were identified and there were no serious threats of inconsistency, imprecision or methodological bias that would further downgrade evidence from a low quality. Where PCI was performed, prehospital 12-lead ECG confirmation was associated with a $39 \%$ reduction in short-term mortality ( 8 studies; $\mathrm{n}=6,339 ; \mathrm{RR} 0.61 ; 95 \% \mathrm{CI}=0.42-0.89 ; \mathrm{p}=0.01 ; \mathrm{I} 2=30 \%$ ) compared to standard or no cardiac monitoring. Where fibrinolysis was performed, prehospital 12-lead ECG confirmation was associated with a $29 \%$ reduction in short-term mortality ( 1 study; $n=17,026$; RR 0.71 ; $95 \% \mathrm{CI}=0.54-0.93 ; \mathrm{p}=0.01$ ). First medical contact-to-balloon, door-toballoon and door-to-needle times were consistently reduced, though large heterogeneity generally precluded pooling. Conclusion: The present study adds to previous reviews by identifying and appraising the strength and quality of a larger body of evidence. Prehospital identification with 12-lead ECG confirmation was found to be associated with reductions in short-term mortality, first medical contact-to-balloon, door-to-balloon and door-to-needle time.

Keywords: STEMI, pre-hospital, 12-lead ECG

\section{OP14}

Implementation and reliability of the Pre-hospital Canadian Triage Acuity Score (Pre-CTAS)

K.E. Smith, BSc, MD, K. Lobay, DMD MD, M. Bullard, MD, University of Alberta, Edmonton, $\mathrm{AB}$

Introduction: With more than a decade of national and international experience, the Canadian Triage and Acuity Scale (CTAS) has been well accepted by emergency department providers. Recently, there has been increasing interest among the Emergency Medical Services (EMS) community in adopting CTAS as a tool to assess patient acuity in the field and to facilitate communication with hospitals receiving the patients into care. The Pre-Hospital Canadian Triage and Acuity Scale (Pre-CTAS) that is based on, and is consistent with, CTAS has been adapted to support those needs. The purpose of this study was to assess interrater reliability of pre-CTAS scores assigned by paramedics on arrival to hospital with CTAS scores assigned by triage nurses. Methods: After pre-CTAS classroom training, EMS providers assigned preCTAS scores, from memory, for all patient transports at two time points: on scene (OS) and at arrival to hospital (AH) for 1 year. The $\mathrm{AH}$ score was compared to the score assigned by the ED triage nurse, who was using computer decision support. Agreement was assessed using Cohen's Kappa scores. Results: 1131 patient transports were included. 195 were excluded (77 did not have 2 Pre-CTAS assigned and 118 had incomplete hospital records). Mean transport time was 20:11 (95\% CI 19:41-20:51). Weighted kappa agreement for EMS At Hospital (AH) vs Triage Nurse CTAS was 0.37 (95\% CI 0.337-0.412). For the 982 patients whose (OS) and (AH) Pre-CTAS were unchanged, EMS-Nurse agreement was 0.406 (0.365-0.447). Conclusion: Implementation of Pre-CTAS was feasible; however, agreement between the scores assigned by EMS and triage nurses (who have access to computer decision support) was only fair to moderate. A more robust implementation strategy and the use of point-of-care decision tools may be required to support the application of Pre-CTAS.

Keywords: CTAS, emergency medical services, triage

OP15

The impact of a novel collaborative long term care - EMS model: a before-and-after cohort analysis

J.L. Jensen, BSc, E.G. Marshall, PhD, A. Carter, MD, M. Boudreau, MA, J. McVey, MD, D.J. Bardua, F. Burge, MD, A. Travers, MD, MSc, Dalhousie University, Halifax, NS

Introduction: An extended care paramedic (ECP) program has been implemented to better address the emergency needs of long term care (LTC) residents. Methods: Data were collected from emergency medical services (EMS), hospital and ten LTC facility databases and patient charts for two five-month time periods, before and after ECP implementation. Outcomes include: number of EMS calls transported to emergency department (ED) and several clinical, safety and system secondary outcomes. Comparisons were made between periods: before (emergency paramedic responses) and after (ECP or emergency paramedics). Statistics included descriptive, chi-squared and t-tests; $=<0.05$. Results: 413 cases were included (before: $\mathrm{n}=136,33 \%$; after $\mathrm{n}=277,67 \%$ ). Median patient age was 85 years (IQR 77-91 years) and $292 / 413(70.7 \%)$ were female. The number calls transported to ED before was 129/136 (94.9\%) and 182/277 (65.7\%) after, p <0.001. In the after period, fewer calls with ECP were transported, compared with calls without ECP: 75/161 (46.6\%) versus 107/116 (92.2\%), p<0.001. Mean EMS call time (dispatch to arrive ED or clear scene) was shorter before than after: 25 minutes versus 36 minutes, $p<0.001$. In the after period, calls with ECP were longer than without ECP: 51 minutes versus 32 minutes, $p=0.004$. The mean patient ED length-of-stay was similar before and after: 7.5 hours compared to 8 hours; $p=$ NS. In the after phase, ED length-of-stay was less with ECPs versus no ECPs: 7.25 hours versus 8.75 hours, $\mathrm{p}=0.047$. Hospital admissions were similar: $39 / 120(32.5 \%)$ versus $77 / 262(29.4 \%), p=0.638$, but in the after phase, fewer patients were admitted with ECP than with no ECP: $32 / 157$ (20.4\%) versus 45/105 (42.9\%), $\mathrm{p}<0.001$. There were zero relapses after no-transport in the before phase and after there were four relapses from 95 calls not transported $(4 / 95,4.2 \%)$; three involved ECP $(3 / 86,3.5 \%)$. Conclusion: ECP reduces the number of LTC residents transported to ED, patient time in ED, and hospital admissions.

Keywords: emergency medical services, long term care, collaboration

OP16

A comparison of different stabilization techniques and transfer methods during pre-hospital management of patients with a suspected neck injury

P. Boissy, PhD, K. Lebel, BEng, M. Charest-Bossé, I. Shrier, MD, $\mathrm{PhD}$, J. Boulay, BSc, DO, E. Segal, MD, S.J. Delaney, MD, C. Vacon, $\mathrm{PhD}$, R.J. Steele, PhD, Department of Surgery, Orthopaedic Service, Université de Sherbrooke, Sherbrooke, QC

Introduction: During pre-hospital management of trauma victims, patients with suspected cervical spine (c-spine) injuries are transferred and secured by paramedics onto a stable surface (e.g. vacuum mattress, spine board) for transport to a medical facility using different stabilization techniques. The objectives of this study were to compare paramedics' ability to minimize c-spine motion during patient transfer onto a vacuum mattress with two stabilization techniques (Head Squeeze vs. Trap Squeeze) and two transfer methods (log roll with one assistant (LR2) vs. 3 assistants (LR4)). Methods: We used a cross-over design with 21 paramedics where each paramedic responsible for c-spine stabilization at the head performed 10 LR2 transfers and 10 LR4 transfers on a simulated patient. For each of the 10 LR2 and 10 LR4 transfers, 
the lead paramedic stabilized the c-spine using the Head Squeeze technique five times and the Trap Squeeze technique five times. We measured relative c-spine motion between the head and trunk using Inertial Measurement Units (IMUs) placed on the forehead and sternum. Our primary outcome was peak change in angular motion (degrees) of the head relative to the trunk from an initial neutral position, calculated directly from data provided by the IMUs. Our secondary outcome was peak angular motion of the head relative to the trunk expressed in each of the six anatomical directions (flexion, extension, right and left lateral flexion, right and left rotation) relative to the initial neutral position. Results: On average, total motion was $3.9^{\circ}$ less with three assistants compared to one assistant $(\mathrm{p}=0.0002)$, and $2.8^{\circ}$ less with the Trap Squeeze compared to the Head Squeeze $(\mathrm{p}=0.002)$. There was no interaction between the transfer method and stabilization technique. When examining specific motions in the six directions, the Trap Squeeze generally produced less lateral flexion and rotation motion but allowed more extension. Examining within paramedic differences, some paramedics were clearly more proficient with the Trap Squeeze technique and others were clearly more proficient with the Head Squeeze technique. Conclusion: Paramedics performing a log roll with three assistants created less motion compared to a log roll with only one assistant, and using the Trap Squeeze stabilization technique resulted in less motion than the Head Squeeze technique. However, large individual differences suggest future paramedic training should incorporate both best evidence practice as well as paramedic preference.

Keywords: suspected neck injury, cervical spine motion, pre-hospital management

\section{OP17}

CHIRPP: a characterization of captured injuries versus uncaptured injuries for patients presenting at a pediatric tertiary care centre

M. Butler, BSc, S. MacPhee, MD, S. Newton, MSc, IWK Health Sciences Centre, Halifax, NS

Introduction: The Canadian Hospitals Injury Reporting and Prevention Program (CHIRPP) is an injury surveillance system that collects detailed information about the nature and cause of injuries in patients presenting to emergency departments across Canada. This program represents a valuable source of information about injury in the Canadian population. As such, it is important to ensure the accuracy of the information reported by CHIRPP. The objective of our study is to describe differences between patients that were not captured by the CHIRPP program at the Izaak Walton Killam Health Sciences Centre in Halifax, and those that were. Methods: This was a retrospective chart review of 2928 patients seen between the January 2013 and June 2013. Patients were identified as either being captured by the CHIRPP program, or not being captured. The groups were evaluated for univariate differences in gender, age distribution, Canadian Triage Assessment Scale (CTAS) score, disposition, and type of activity engaged in during time of injury. Logistic regression models were built to evaluate for factors that affect on the probability of capture into the CHIRPP program. Effects are described using odd ratios, $95 \%$ confidence intervals, and p-values. Validation of individual models was done using the Hosmer-Lemenshow Test. Results: The uncaptured group tended to have more young patients ( $0-4$ years) and older patients (15-19 years). $36.8 \%$ of uncaptured patients were assessed as a CTA I to III as opposed to $20 \%$ for captured patients. Uncaptured patients were more likely to be leave without being seen, and to be admitted (OR=2.645, CI: $1.394,5.08), \mathrm{P}=0.003)$. The activities associated the most with non-capture were self-harm ( $\mathrm{OR}=12.68, \mathrm{CI}$ : $(3.11,63.98), \mathrm{P}=0.001)$, drugs/overdose (OR=4.79, CI: $(1.43,18.2), \mathrm{P}=0.014)$ and foreign bodies in ears $(\mathrm{OR}=8.46, \mathrm{CI}:(1.72,52.82), \mathrm{P}=0.013)$. Conclusion: Patients that are assessed as more severe $(\mathrm{CTAS}>4)$ and patients that were admitted were more likely to not be captured by the CHIRPP program. In addition, patients that presented as cases of self-harm or drug overdose were less likely to be captured by the program. This may be an indication that CHIRPP may under-represent more serious injuries, as well as the number of cases of self-harm and drug ingestion. This revelation can be used to inform local policy and better understand injury patterns in Nova Scotia.

Keywords: injury

\section{OP18}

Canadian CT-Head Rule criteria are not predictive of long-term complications in $\mathrm{mTBI}$

P. Ouellet, MD MSc, N. Le Sage, MD, MSc, X. Neveu, MSc, M. Emond, MD, MSc, P. Chabot, Inf., McGill University, Montréal, QC

Introduction: Postconcussive symptoms (PCS) constitute an important complication of mild traumatic brain injury (mTBI). The Canadian CT-Head Rule (CCHR) criteria have never been studied as individual predictors of long-term complications of mTBI. We hypothesize that clinical variables used in the CCHR, predictive of short term mTBI complications are not associated with complications in the form of PCS at 1 week or 3 months. Methods: This was a prospective cohort study set in 5 emergency departments (ED) across the province of Québec that included 379 adult mTBI patients. The variables of interest were: (1) GCS score $<15$ two hours after injury, (2) signs of open or depressed skull fracture, (3) signs of skull base fracture, (4) $\geq 2$ episodes of vomiting, (5) age $\geq 65$, (6) retrograde amnesia for more than 30 minutes, and (7) dangerous mechanism of injury. The primary outcome was the presence of PCS symptoms at 1 week and 3 months. Association of each individual CCHR criterion with PCS was achieved by relative risks (RR) and their $95 \%$ confidence intervals (CI). Results: At 1 week, only a GCS less than 15 at 2 hours following injury was associated with an increased risk of PCS. All the other criteria did not show a significant association with PCS (signs of depressed skull fracture (RR 1.40; CI 1.18 - 1.67), signs of skull base fracture (RR 0.81; CI 0.36 - 1.80), vomiting $\geq 2$ (RR 0.88; CI 0.51 - 1.51), age $\geq 65$ (RR 0.76; CI $0.52-1.12$ ), retrograde amnesia for more than 30 minutes (RR 1.07; CI 0.85 - 1.36), and dangerous mechanism (RR 0.92 ; CI 0.75 - 1.12).) At 3 months, no variable was associated with an increased risk of PCS (GCS less than 15 at 2 hours following injury (RR 0.74; CI 0.40 - 1.39), signs of depressed skull fracture (RR 0.53; CI 0.09 - 3.19), signs of skull base fracture (RR 0.64; CI 0.11 - 3.72) vomiting $\geq 2$ times (RR 1.35; CI 0.68 - 2.69), age $\geq 65$ (RR 1.24; CI 0.76 - 2.03), retrograde amnesia for more than 30 minutes (RR 1.20; CI $0.78-1.85$ ), and dangerous mechanism (RR 0.99; CI 0.68 - 1.43).) Conclusion: Individual CCHR criteria are not associated with presence of PCS at 1 week or at 3 months. Only GCS less than 15 two hours after injury was associated with PCS at 1 week. This study demonstrates that CCHR criteria cannot be used as predictors of long term complications in mTBI. Specific early predictors of PCS in the ED need to be investigated to better orient mTBI patients' disposition. Keywords: mild traumatic brain injury, clinical prediction, post concussion symptoms

\section{OP19}

Compressions during defibrillator charging shortens shock pause duration and improves chest compression fraction during shockable out of hospital cardiac arrest

S. Cheskes, MD, M.R. Common, BSc, A. Byers, BSc, C. Zhan, MSc, L.J. Morrison, MD, MSc, University of Toronto, Toronto, ON

Introduction: Previous studies have demonstrated significant relationships between shock pause duration and survival to hospital discharge from out-of hospital (OHCA) shockable cardiac arrest. Com- 
pressions during defibrillator charging (CDC) has been proposed as a technique to shorten shock pause duration. We sought to determine the impact of CDC on shock pause duration and CPR quality measures in shockable OHCA. Methods: We performed a retrospective review of all treated adult OHCA occurring over a one-year period beginning August 1, 2011 after training EMS agencies in CDC. We included OHCA patients with an initial shockable rhythm, available CPR process and shock pause data for the first three shocks of the resuscitation. CDC by EMS personnel was confirmed by review of impedance channel measures. We evaluated the impact of CDC on shock pause duration as the primary outcome measure. Secondary outcome measures were the impact of CDC on CPR quality measures. Results: Among 674 treated OHCA 158 (23.4\%) presented in a shockable rhythm of which 129 (81.6\%) met study inclusion criteria. Seventy $(54.2 \%)$ received CDC. There was no significant difference between the $\mathrm{CDC}$ and no $\mathrm{CDC}$ group with respect to Utstein variables. Mean pre-shock pause ( 24.3 vs. $8.7 \mathrm{sec} ; 15.6 ; 95 \%$ CI: 12.7 , 18.5 ), post-shock pause ( 6.0 vs. $3.8 \mathrm{sec} ; 2.2 ; 95 \% \mathrm{CI}: 0.8,3.5)$, and peri-shock pause (30.3 vs. $12.3 \mathrm{sec} ; 18.0 ; 95 \%$ CI: $14.7,21.3)$ were all significantly lower for those who received CDC. Mean chest compression fraction was significantly greater ( 0.77 vs. $0.70,0.07 ; 95 \%$ CI: $0.03,0.11)$ with CDC. No significant difference was noted in compression rate or depth with CDC. Clinical outcomes did not differ between the two approaches (return of spontaneous circulation $62 \%$ vs. $62 \% \mathrm{p}=0.98$, survival $25.4 \%$ vs. $27.1 \% \mathrm{p}=0.82$ ), although the study was not powered to detect clinical outcome differences. Conclusion: Compressions during defibrillator charging shortens shock pause duration and improves chest compression fraction in shockable OHCA. Given the impact on shock pause duration, further study with a larger sample size is required to determine the impact of this technique on clinical outcomes from shockable OHCA.

Keywords: cardiopulmonary resuscitation, resuscitation, survival

\section{OP20}

The impact of chest compression release velocity on outcomes from out-of-hospital cardiac arrest

S. Cheskes, MD, A. Byers, BSc, C. Zhan, MSc, A. Silver, PhD, L.J. Morrison, MD, MSc, University of Toronto, Toronto, ON

Introduction: Previous studies have demonstrated significant relationships between CPR quality metrics and survival to hospital discharge from out-of hospital (OHCA) cardiac arrest. Recently a new metric, chest compression release velocity (CCRV) has been associated with improved survival from OHCA to determine the impact of CCRV on clinical outcomes from OHCA. Methods: We performed a retrospective review of prospectively collected data on all treated adult OHCA occurring over a one-year period (Jan 2012 - Jan 2013) in two Canadian EMS agencies. CPR metrics of chest compression fraction (CCF), compression rate, compression depth, shock pause duration and CCRV were abstracted from impedance channel measurements during each resuscitation. Cases of public access defibrillation, EMS witnessed arrest and those missing any Utstein variable or discharge status data were excluded. We performed a multivariable regression analysis to determine the impact of CCRV on survival to hospital discharge. Secondary outcome measures were the impact of CCRV on return of spontaneous circulation (ROSC) and neurologically intact survival (MRS < 3). Results: Among 908 treated OHCA, 611 met inclusion criteria. The median (IQR) age was $71.7(60.7,81.6)$ with $395(64.6 \%)$ being male. $140(22.9 \%)$ presented in ventricular fibrillation, $122(20 \%)$ pulseless electrical activity and $349(57.1 \%)$ asystole. The median (IQR) CPR quality metrics were: CCF $0.81(0.73,0.85)$, compression rate $105 /$ minute $(101,115)$, compression depth $49.9 \mathrm{~mm}(42.5,56.7)$, preshock pause $13.5 \operatorname{secs}(8,19)$ and post-shock pause $3.5 \operatorname{secs}(2.8,5)$.
The median (IQR) CCRV (mm/sec) amongst 49 survivors was 135.9 $(115.4,156.5)$ compared to $120(102.9,140)$ in 562 non survivors $(p=0.009)$. When adjusted for CPR metrics and Utstein variables, the odds of survival to hospital discharge for each $5 \mathrm{~mm} / \mathrm{sec}$ increase in CCRV was 1.02 (95\% CI: 0.97, 1.08). Similarly the odds of ROSC and neurologically intact survival were 1.02 (95\% CI: $0.99,1.05)$ and 1.03 (95\% CI: 0.98, 1.08), respectively. Conclusion: When adjusted for Utstein variables and CPR quality metrics, CCRV was not significantly associated with outcomes from OHCA. Our findings may have been impacted by the overall survival rate in our study cohort.

Keywords: cardiopulmonary resuscitation, resuscitation, survival

\section{OP21}

Gender differences in access to post-arrest care: a SPARC network cohort study

L.J. Morrison, MD, MSc, V.E. Rac, MD, PhD, P. Dorian, MDCM, MSc, S. Cheskes, MD, R. Fowler, MD, MDCM, MSc, K.N. Dainty, MSc, PhD, M. Gaudio, BSc., E. Racz, BHSc, BA, J. Parsons, PhD, A. Kiss, PhD, A. Slutsky, MD, PhD, A.S. Bierman, MD, MSc, B.L. Abramson, MD, MSc, S.H. Gray, MD, D.C. Scales, MD, PhD, Division of Emergency Medicine, Department of Medicine, University of Toronto, Toronto, ON

Introduction: To explore gender disparities in the delivery of targeted post-arrest resuscitation care interventions after out-of-hospital cardiac arrests (OHCA) in a prospective Canadian data set of OHCA presenting to 32 hospitals participating in the Strategies for Post-Arrest Care (SPARC) Network. Methods: A prospective cohort study of consecutive adult OHCA of no obvious cause with return of spontaneous circulation for at least 20 minutes, without an advance directive in place, presenting to the 32 hospitals participating in the Strategies for PostArrest Care Network. The primary outcome was the proportion of post-OHCA patients receiving angiography with or without percutaneous coronary intervention (PCI) within 90 minutes of documented ST-elevation myocardial infarction (STEMI) on 12-lead ECG on admission to hospital. Secondary measures included rates of targeted temperature management (TTM) and withdrawal of life sustaining therapy. Results: Of the 1053 treated adult OHCAs meeting the inclusion criteria, 336 were women and 717 were men with mean ages 68 (17) women vs. 66 (15) male, $p=0.07$. Woman were less likely to have favourable Utstein predictors of survival; $\mathrm{p}<0.02$ and STEMI postarrest; 58 [18\%] women vs. 208 [30\%] male $p<0.0001$. There was no observed gender difference between achieving the time target for angiography with or without PCI for STEMI positive; 18 [31\%] vs. 79 $[38 \%] \mathrm{p}=0.33$. Women who were STEMI negative were less likely to receive coronary angiography; $22[8 \%]$ vs. $74[15 \%] \mathrm{p}=0.006$ ) or PCI; $7[3 \%]$ vs. $30[6 \%] \mathrm{p}=0.04$. Eligible women were less likely to receive TTM; $147[78 \%]$ vs. 382 [85\%] p=0.04. Women were more likely to have life sustaining therapy withdrawn at any time; 127 [38\%] vs. 221 [31\%] $\mathrm{p}=0.03$ and within 72 hours; 78 [61\%] vs. $109[49 \%] \mathrm{p}=0.03$. Women were less likely to survive to discharge; $0.67,95 \%$ CI $0.5,0.9$, $\mathrm{p}=0.005$ however this difference disappeared when adjusted for Utstein predictors associated with survival which were more common in men; OR $1.295 \%$ CI 0.9 to $1.6, p=0.32$. Conclusion: OHCA in women was associated with unfavourable predictors of survival, increased rates of early withdrawal of life sustaining therap, reduced rates of TTM and less diagnostic testing when STEMI negative on ECG. Despite these differences, adjusted survival was similar for both genders. These observations suggest that women who suffer OHCA survive in spite of the unfavourable predictors of outcome and the lack of intervention post arrest.

Keywords: resuscitation guidelines, implementation science network, gender differences in post arrest care 
OP22

Barriers to self-reporting patient safety events by paramedics J.E. Sinclair, MScN, C.J. Bourque, MBA, L.A. Calder, MD, MSc, M. Austin, MD, A. Reed, MD, J. Kortko, BSc, J. Maloney, MD, Regional Paramedic Program for Eastern Ontario, Ottawa Hospital, Ottawa, ON

Introduction: Strategic priorities to address patient safety issues in EMS have recently been emphasized, however, little research exists examining the extent to which patient safety events occur within EMS and even fewer studies investigate patient safety systems for selfreporting by paramedics. The purpose of this study was to identify the barriers to paramedic self-reporting of patient safety events; specifically, patient care variances (PCVs), near misses and adverse events. Methods: The survey listed 18 potential barriers to self-reporting, including 11 fear-based barriers. They were presented for rating on a 5 point Likert scale (very significant - very insignificant). Five different patient safety event clinical scenarios (near miss, adverse event, minor, major and critical PCV) were randomly distributed to 1153 paramedics during the 2012 continuing medical education sessions. Data analysis consisted of descriptive statistics, chi-square tests and Mann-Whitney tests, where applicable. Results: We received responses from 1133 paramedics (98.3\%). Almost one third (28.2\%) were Advanced Care Paramedics and $45.0 \%$ had more than 10 years' experience. The top five barriers to self-reporting (very significant or significant) (n, \%) were: fear of being punished $(905,79.9 \%)$, fear of being suspended and short-term income loss $(890,78.6 \%)$, fear of a Ministry of Health and Long Term Care (MoHLTC) investigation (882, 77.9\%), fear of termination and long-term income loss $(880,77.7 \%)$, and fear of deactivation $(869,76.7 \%)$. Overall, $64.2 \%$ responded they would selfreport the patient safety event. Intention to self-report varied according to the patient safety event clinical scenario (22.3\% near miss, $46.0 \%$ adverse event, $73.3 \%$ minor PCV, $85.7 \%$ major PCV, $93.2 \%$ critical $\mathrm{PCV})$. No association was found between the scope of practice and intention to self-report $(\mathrm{p}=0.55)$. Conclusion: A high proportion of fear-based barriers exist to self-reporting of patient safety events suggesting that a culture change is needed to facilitate the identification of future patient safety threats.

Keywords: patient safety, paramedic, self-reporting

OP23

Safety evaluation of the Canadian C-Spine Rule for selective cspine immobilization by paramedics in the field

C. Vaillancourt, MD, MSc, M. Charette, MSc, J. Sinclair, MScN, G. Wells, PhD, I.G. Stiell, MD, MSc, Ottawa Hospital Research Institute, Ottawa, ON

Introduction: The Canadian C-Spine Rule (CCR) for radiography in alert and stable trauma patients was previously validated by physicians in a cohort of 8,283 patients, and by paramedics in 1,949 additional patients. The CCR calls for evaluation of active neck rotation if patients have none of 3 high-risk criteria and at least 1 of 5 low-risk criteria. This study evaluated the safety and potential impact of an active strategy that allows advanced and primary care paramedics to assess very low-risk trauma patients with the CCR and transport them without immobilization. Methods: This prospective cohort implementation study took place in Ottawa and involved alert (GCS 15) and stable adult trauma patients at risk for neck injury. Paramedics completed standardized data forms and interpreted the CCR status for all patients. Paramedics were empowered by a medical directive to not immobilize the c-spine of selected patients according to the CCR, thus allowing them to rapidly transport patients to the emergency department. Some patients were independently examined by a second paramedic. We reviewed diagnostic imaging performed at 11 receiving hospitals for clinically important c-spine injury (main outcome). We followed all patients without initial radiologic evaluation for 30 days for referral to our spine service, or subsequent visit with radiologic evaluation. Analyses included sensitivity, specificity, kappa coefficient, and descriptive statistics with 95\% CIs. Results: The 2,981 patients enrolled over 3 years had these characteristics: mean age 42 (range 1694), female $51.6 \%$, motor vehicle collision $54.8 \%$, fall $19.9 \%$, admitted to the hospital $7.6 \%$, acute c-spine injury $0.9 \%$, clinically important cspine injury $0.4 \%$. The CCR classified patients for 8 important injuries with sensitivity $100 \%$ (95\%CI 67.6-100), specificity $67.8 \%$ (95\%CI 65.8-69.7). The kappa values for interpretation of the CCR between paramedics and investigators, and among 66 paramedics were 0.92 (95\%CI 0.91-0.94) and 0.81 (95\%CI 0.66-0.95). Paramedics were comfortable applying the rule in $90.5 \%$ of cases. Scene time was 17 min for those not immobilized compared to $21 \mathrm{~min}$ for those who were. Four minutes were saved and 2,046 prehospital immobilizations were avoided using the CCR. Conclusion: Paramedics can apply the CCR with accuracy and identified all important cervical-spine injuries. The adoption of the CCR by paramedics had a significant impact on the number of prehospital immobilizations.

Keywords: clinical decision rule, c-spine injury, emergency medical services

OP24

Sex differences in presentations to emergency departments in Alberta, Canada for atrial fibrillation/flutter: a provincial population-based cohort study

R.J. Rosychuk, PhD, M. Graham, MD, B.H. Rowe, MD, MSc, B.R. Holroyd, MD, University of Alberta, Edmonton, AB

Introduction: Differences in atrial fibrillation/flutter (AFF) incidence and management may exist for different subgroups of the population and their presence and reasons need to be understood. We sought to examine the differences between male and female presentations to EDs for AFF, and subsequent post-ED physician visits in the province of Alberta, Canada. Methods: The Ambulatory Care Classification System and provincial administrative databases were used to identify all primary ED encounters for AFF during 12 fiscal years (April 1999 to March 2011) for patients > 35 years of age. Information extracted included demographics, ED visit timing, and subsequent visits to nonED settings. Data analysis included descriptive summaries and directly standardized visit rates (DSVR). Results: There were 63,398 ED presentations for AFF (48.2\% female) made by 32,104 patients $(15,420$ females $[48.0 \%])$. The majority of ED presentations ended in discharge (20,637 [67.6\%] for females). For particular age, socio-economic, and regional groups, females were less likely to be discharged, had fewer follow-up visits (non-ED setting) with cardiologists within 90 days of the ED presentation, and had longer times to first follow-up visit and to first follow-up visit with a cardiologist than their male counterparts. Conclusion: Variation exists with respect to the care provided to women with AFF. The differences seen for discharge rates, number of cardiology follow-up visits, and time to first cardiology follow-up visit are not solely based on the longevity of women. Further understanding these differences could lead to specific initiatives to address any bias in services to females.

Keywords: atrial fibrillation, epidemiology, emergency department

OP25

Echo-Guided Life support (EGLS): an algorithmic approach to the patient in shock

M. Valois, MD, J. Lanctôt, MD, McGill University, Montréal, QC

Innovation concept: Point-of-care ultrasound is pertinent to the evaluation of the patient in shock in many ways. For non-experts confusion can arise from the many possibilities available to evaluate a shock 
patient. The EGLS approach is designed to standardise and facilitate its use in a potentially stressful situation and to optimise the examination sequence. (References: http://link.springer.com/article/10.1007/ s13089-011-0083-2). It is designed to cover the main etiologies of shock and avoid certain pitfalls of ultrasound evaluation. An assessment of core competencies in point-of-care ultrasound is currently being built and aims at evaluating the acquisition, interpretation and clinical integration through a written exam, OSATS (objective structured assessment of technical skills) and CRM (crisis response management). Methods: The training is designed by 2 hour modules on each component of the algorithm : inferior vena cava assessment, focused echocardiography (subcostal, parasternal long and short and apical views) combined with a session on physiology (venous return and cardiac output curves) and ACLS-like case scenarios that allow participants to integrate their learning and easily allows evaluation. All participants have to read prior documentation and view online podcasts and conferences. Questions at that point are answered by email or via twitter. The course includes an iPhone/iPad application given to the participants that subsequently serves as a reminder and also as a template allowing real-time bedside comparison of the patient's findings to standardized videos on the application thus allowing in-time teaching and learning. Curriculum, tool, or material: The bedside clinical access to a dynamic template allows for better ease of application of the notions learned at the course and help in getting over the associated learning curve. The course on Echo-guided Life Support has been given to over 350 physicians in Canada. It will be incorporated in undergraduate studies at McGill University Conclusion: The use of reverse learning techniques coupled with the use of dynamic templates (In-time learning and recall) enhances the probability that participants will use the techniques acquired at the course once at the patient's bedside.

Keywords: innovations in EM education, ultrasound, shock

\section{OP26}

Presentations to emergency departments in Alberta, Canada for atrial fibrillation and flutter: a large population-based study

R.J. Rosychuk, PhD, B.H. Rowe, MD, MSc, M. Graham, MD, B.R. Holroyd, MD, Alberta Health Services, Edmonton, AB

Introduction: Little is known of the pattern of presentations to an emergency department (ED) setting by patients with atrial fibrillation or flutter (AFF). This study was designed to describe of AFF presentations in a single Canadian province. Methods: The Ambulatory Care Classification System and provincial administrative databases were used to obtain all primary ED encounters for AFF during 12 fiscal years (April 1999 to March 2011) for patients > 35 years of age. Information extracted included demographics, ED visit timing, and subsequent visits to non-ED settings. Data analysis included descriptive summaries and directly standardized visit rates (DSVR). Results: Overall, there were 63,398 ED AFF visits made by 32,104 distinct adults. Median ages for females and males were 75 and 67 years, respectively; more men $(52 \%)$ and patients $>65$ presented. Overall, the standardized rates declined from 292 to 280 per 1,000 over the study period. Specific populations of social-assistance recipients and Aboriginals had higher ED visit rates for AFF than other groups. Predictable daily, weekly, and monthly trends were observed. The ED visits were followed by numerous subsequent visits in non-ED settings; however, Aboriginals and women had lower Cardiology follow-up. Conclusion: AFF is a common presenting problem in Alberta EDs. The important findings include an overall decrease in the rates of presentation over the study; however, disparities exist based on age, socio-economic/cultural and sex status exists. Further understanding these differences could lead to specific initiatives to address any bias in management and services that exist.

Keywords: atrial fibrillation, epidemiology, emergency department
OP27

The utility of measuring international normalized ratio (INR) as part of the investigation of patients with cardiac-type chest pain S.G. Campbell, MB BCh, K. Magee, MD, FRCPC, I. Cajee, MBBCh, S. Field, MB BCH, M. Butler, BSc, C.L. Campbell, MB BS, Dalhousie University, Halifax, NS

Introduction: Many laboratory tests are ordered in ED patients as a matter of 'routine'. Many of these are of questionable value in improving patient outcome, yet add significant cost to the system. A frequent test in patients with chest pain suspected to be cardiac in origin is the INR, a standardized measurement of the prothrombin time, a measure of the extrinsic pathway of coagulation. This test is rarely abnormal in patients not on anticoagulant medication, and without liver disease or inherited coagulation deficiency. We aimed to identify if there was a subset of this group of patients (beside those mentioned) in whom INR testing was a relevant component of workup. Methods: This retrospective chart review involved medical records of 984 patients who had been referred to cardiology for the investigation of chest pain. Patients on warfarin were excluded leaving 935 patients for review. Clinical details and INR results were reviewed independently by four experienced ED faculty (average 25,5 yrs experience, range 18-37). Reviewers indicated if they thought the INR result was relevant to ED care. A result was considered 'relevant' if any of the reviewers considered it so. Results: INR was measured in 736 patients (78.7\%). 13 (1.8\%) patients had relevant abnormal INR results. Subsequent inquiry found that one had a known coagulation anomaly (Factor X deficiency), three were on other anticoagulants (2 dibigitran and one clopidigrel, all three with IRN's marginally abnormal), and in 9, their active use of warfarin had not been recorded in the chart. No INR findings were found to contribute to the emergency care of these patients. Conclusion: Routine INR testing is commonly conducted in the ED on patients with cardiac-type chest pain; Results of the test add little to emergency care in patients without the usual (hematologic) indications for the test. INR testing for patients with chest pain suspected to be cardiac in origin should be restricted to patients on non-ASA anticoagulants, with liver disease or with known coagulation abnormalities.

Keywords: prothrombin time, INR, chest pain

\section{OP28}

The relationship of maximum troponin values post out-of-hospital cardiac arrest with electrocardiographic findings, cardiac procedures and survival to discharge; a sub-study of ROC PRIMED

L.J. Morrison, MD, MSc, S.M. Devlin, PhD, M.C. Kontos, MD, S. Cheskes, MD, I.G. Stiell, MD, MSc, A.J. Thomas, MPH, J.P. Ornato, MD, J. Christenson, MD, V.E. Rac, MD, PhD, T.P. Aufderheide, MD, J.G. Wigginton, MD, P. Dorian, MDCM, MSc, Rescu, Li Ka Shing Knowledge Institute, St. Michael's Hospital, University of Toronto, Toronto, ON

Introduction: To describe the relationship between maximum Troponin (Tn) levels recorded within 48 hours post arrest and clinical outcomes in out-of-hospital cardiac arrest (OHCA) patients enrolled in the Resuscitation Outcomes Consortium (ROC) Prehospital Resuscitation using an IMpedance valve and Early versus Delayed analysis (PRIMED) trial. Methods: A prospective observational cohort study of all treated non-traumatic OHCAs enrolled in ROC PRIMED. Patients were classified based on first presenting in-hospital ECG as ST elevation myocardial infarction (STEMI) or not a STEMI (including NSTEMI). Peak Tn levels, evaluated on a logarithmic scale, were compared across patient and treatment characteristics using a t-test or ANOVA. The association between categories of Tn levels $(<0.1,0.1-2$, $>2$ ) and survival to discharge was evaluated using logistic regression 
adjusted for Utstein predictors of survival and ROC site. Results: Of the 15617 enrolled patients 3661 (23\%) survived at least 48 hours; $17 \%$ (693) were STEMI and 78\% (3188) were not a STEMI with 5\% unknown and $83 \%$ (3460) with at least one Tn level measured. The mean (SD) age was 64.6 (15.9). The STEMI group had more men (74.5\% STEMI, $62 \%$ not a STEMI) and was more likely to have an initial shockable rhythm (73\% STEMI, 39\% not a STEMI). In-hospital survival was higher with STEMI (OR 1.93 95\%CI 1.63, 2.3, p<0.001). The logarithm of Tn values was higher in STEMI patients $(\mathrm{p}<0.001)$ (Figure 1). Adjusted in-hospital survival for STEMI patients was significantly better in those patients with higher Tn values $(\mathrm{p}=0.01)$. Adjusted in-hospital survival was significantly worse with higher Tn levels in the not a STEMI group $(\mathrm{p}<0.001)$ When treated with reperfusion, adjusted survival in the not a STEMI group was significantly better than the survival rate without reperfusion (OR 3.6, 95\% CI 2.4, 5.4 for trop $>0.1 \mathrm{p}<0.001)$ Conclusion: High Tn levels were associated with increased rates of reperfusion and better in-hospital survival in post arrest patients with STEMI on first ECG. High Tn levels in not a STEMI patients post arrest were associated with decreased survival. Survival in not a STEMI patients was significantly higher when treated with reperfusion.

Keywords: post arrest care, biochemical marker utility post arrest, resuscitation guidelines

\section{OP29}

Discharged minor thoracic injuries patients have functional limitations

M. Emond, MD, MSc, J. Chauny, MD, MSc, L. Vanier, MD PhD, N. Le Sage, MD, MSc, Université de Montréal, Montréal, QC

Introduction: Approximately $75 \%$ of patients with a minor thoracic injury are discharged after an emergency department visit, while significant functional limitations can occur in the weeks that follow. Objective: To investigate whether minor thoracic injury and determinants relate to subsequent functional limitations. Methods: A prospective 19-months cohort study with a 90-day follow-up was conducted in four university-affiliated emergency departments. Patients 16 years and older with an minor thoracic injury with or without rib fracture, who presented between March 1st, 2008 and October 1st, 2009, were recruited and assessed at initial emergency department visit, 14, 30 and 90 days post-injury. The main outcome measure(s). Functional outcome was assessed using the SF-12 scale at 30 and 90 days post-injury. Univariate and multivariate analyses were realized to assess determinants of functional limitation. Results: Of the 482 recruited participants, $127(26.3 \%)$ were aged 65 or more. Overall, 147 patients $(30.5 \%)$ presented with at least one rib fracture and 59 subjects $(12.2 \%)$ with delayed hemothorax. At 90 days, $22.8 \%$ of patients still had severe or moderate disabilities on global physical health score. Patients with solely delayed hemothorax and no rib fracture had the lowest global physical health score (46.4 vs 61.1, $\mathrm{p}<0.01$, effect size $=-$ 2.56) compared to patients with simple minor thoracic injury. Their physical functioning score were also lower up to 90 days post-injury (72.1 vs $85.3, \mathrm{p}<0.01$, effect size $=-1.21$ ). Generally, functional limitations also increase with increments of number of rib fracture detected on radiograph. Outcomes were not different amongst patients aged 65 years or more when compared to their younger counterparts. Conclusion: In this prospective study, severe to moderate disabilities were present in one patient out of five. The presence of delayed hemothorax and the number of rib fracture were associated with increased functional limitations after a minor thoracic injury. Physicians should address detection of delayed hemothorax and prevention of disability in their discharge instructions.

Keywords: functional limitation, minor thoracic injury
OP30

Epidemiology and clinical predictors of biphasic reactions in children with anaphylaxis

W. Alqrashi, MD, I.G. Stiell, MD, MSc, K. Chan, MD, G. Neto, MD, G. Wells, PhD, CHEO, Ottawa, ON

Introduction: Biphasic reaction is the recurrence of anaphylactic symptoms after initial resolution despite no further exposure to the trigger. Epidemiological data regarding biphasic reactions among children is sparse. This study aims to investigate the prevalence and clinical predictors for the biphasic reaction in children presenting to the Emergency Department (ED) with anaphylaxis. Methods: A multicenter retrospective cohort study was conducted for ED visits from January - December 2010. All visits that satisfied anaphylaxis diagnostic criteria of the National Institute of Allergy and Infectious Disease Food Allergy and Anaphylaxis Network (NIAID/FAAN) were included. Data was collected on patient demographics, inciting allergens, symptoms, management, length of stay, incidence of biphasic reactions and disposition. A second reviewer extracted data from $10 \%$ of the ED records. We analyzed the predictors of biphasic reaction using univariate analysis and multiple logistic regression. Results: Out of 1,749 ED records reviewed, 484 visits fulfilled study inclusion criteria. Median age was 4.8 years (IQR 2.1-11.1) and 314 (64.9\%) were male. Seventy one $(14.7 \%)$ patients developed biphasic reactions. Among those who had biphasic reactions, $35(49.3 \%)$ the biphasic reaction was treated with epinephrine, and $53(74.5 \%)$ developed the reaction before ED discharge with a median time of 4.7 hours (IQR 3.3-6) from onset of the initial reaction to onset of the biphasic reaction. We found five independent predictors of biphasic reaction: age 6-9 years (OR 3.60; $95 \%$ CI 1.5-8.58), time from onset of the anaphylactic reaction to ED presentation $>90$ minutes (OR 2.58; 95\%CI 1.47-4.53), wide pulse pressure at triage* (OR 2.92; 95\% $\mathrm{CI} 1.69-5.04)$, treatment of the reaction with $>1$ dose of epinephrine (OR 2.7; 95\%CI 1.12-6.55), and administration of inhaled Salbutamol in ED (OR 2.39; 95\%CI 1.244.62). There was no association between treatment of the initial anaphylactic reaction with systemic steroid or $\mathrm{H} 2$-antihistamine and development of biphasic reactions. Conclusion: Biphasic reactions seem to be associated with more severe anaphylactic reactions. We identified five clinical predictors that could ultimately be used to identify patients who would benefit from prolong ED or inpatient monitoring. In agreement with previous literature, we found no benefit of systemic steroid or $\mathrm{H} 2$-antihistamine in preventing biphasic reactions. These findings may enable better utilization of ED resources and counseling of patients and families after anaphylactic reactions.

Keywords: anaphylaxis, biphasic reaction, pediatric

OP31

Estimating pediatric weights in the emergency department: when we guess wrong, what are the implications?

J. Ferguson, MD, R. Sadoway, Bachelor of Arts, A. Finningley, BSc, R. Woods, MD MMEd, University of Saskatchewan, Saskatoon, SK

Introduction: In the Emergency Department (ED) medications used in resuscitation of pediatric patients are often dosed based on estimated weights calculated using age or length. Our team evaluated the accuracy of common weight estimation methods in a pediatric population and solicited expert opinion on when over/under-dosing may cause harm in resuscitation scenarios. Methods: A convenience sample of patients presenting to the Pediatric ED at Royal University Hospital was enrolled between June 1 - August 1, 2012. Patient length, weight and demographics were recorded. Seven age-based methods were used to calculate weight and their percentage error was determined. The number of patients with concordant and discordant Broselow color cate- 
gories was determined. Seven experts in pediatric resuscitation participated in a survey and focus group to determine the percent over/underdose that may cause harm for twenty commonly used medications based on five resuscitation scenarios. Results: 226 female and 275 male patients aged 2 days to 17 years (median 3.36 years) were enrolled. The seven age-based methods resulted in percentage errors ranging from $8.5 \%$ to $14.2 \%$. The Broselow Tape was concordant in $66.5 \%$ (2007) and $66.7 \%$ (2011) of patients. The most significant outliers from the Broselow Tape had overestimations of $105 \%$ and underestimations of $73 \%$. On average, one and two weight category discrepancies in Broselow category resulted in overestimating weight by $68.9 \%$ and $112.0 \%$ or underestimating weight by $39.3 \%$ and $51.3 \%$. The expert focus group concluded that harm may occur with overdoses greater than $100 \%$. The exceptions to this were Propofol and Ketamine in cases with hypotension where the group felt that even a full dose may cause harm. It was felt that harm may occur when underdosing medications between $5-100 \%$. Antibiotics were the most important medications not to underdose. The focus group recommended titrating medications and generally felt that it was safer for patients to be overdosed than underdosed. Conclusion: Most methods of pediatric weight estimation are reasonably accurate. The Broselow Tape will estimate the correct weight category two thirds of the time. When weight estimations are inaccurate, patients are unlikely to experience harm unless there is a significant over/under-dose. We recommend that clinicians be particularly careful not to overdose sedatives or underdose antibiotics.

Keywords: resuscitation, pediatrics, weight estimation

\section{OP32}

Remifentanil for procedural sedation: a systematic review

M. Kisilewicz, MD, H. Rosenberg, MD, C. Vaillancourt, MD, MSc, University of Ottawa, Department of Emergency Medicine, Ottawa, $\mathrm{ON}$

Introduction: Remifentanil is an ultra-short-acting opiate with possible advantages over the emergency physician's current arsenal for procedural sedation (PSA). We sought to determine the performance characteristics of remifentanil as a PSA agent for procedures similar to those executed in the emergency department (ED). Methods: We systematically reviewed MEDLINE, PubMed, EMBASE, conference proceedings, and trial registries. We included peer-reviewed studies that used remifentanil as a PSA agent and in which the procedure(s) being performed and the setting were relevant to the ED. Outcome measures included procedural success, recovery time, patient and physician satisfaction, patient safety, and resource management. Two trained authors independently performed article selection and completed piloted data abstraction forms. We assessed inter-observer agreement using kappa statistics $(\mathrm{K})$, and explored the validity of selected randomized control trials (RCT) using the Cochrane Risk of Bias Assessment Tool. We performed narrative summary analyses. Heterogeneity among selected studies precluded meta-analysis. Results: The search strategy identified 1,258 citations; 31 were selected for full review $(=0.64)$, and 7 met predetermined inclusion criteria $(=0.62)$ : 4 RCTs, 1 prospective cohort study, 1 health record review, and 1 case series. Three studies took place within the ED, and 4 took place in clinics. Procedures included lumbar puncture (80), cardioversion (66), orthopedic manipulation (63), incision and drainage (15), thoracostomy (8), and nasal packing (2). There was extensive variation in remifentanil dosing $(0.15-1.5 \mathrm{ug} / \mathrm{kg})$, administration protocols, and use of additional PSA drugs. All studies performed in the ED noted faster procedure completion, recovery, and discharge times with remifentanil compared to control groups. Higher rates of respiratory depression occurred in one adult RCT (2.4 vs 6.0\%). One small pediatric study, reported $85 \%$ of patients required verbal stimulation for resolution of apneas. Three children also developed anxiety, one such episode necessitating a period of positive pressure venti- lation. No other serious adverse event was reported. All studies were found to carry significant risk of bias. Conclusion: There currently is a lack of high quality data supporting the routine use of remifentanil in the ED. However, faster recovery times and the absence of serious adverse events could justify continued investigations.

Keywords: remifentanil, procedural sedation

\section{OP33}

Treatment and reduction of anxiety and pain in pediatric emergency department: 'TRAPPED' survey; a pediatric emergency research Canada study

E. D. Trottier, MD, J. Gravel, MD, MSc, S. LeMay, PhD, S. Ali, MDCM, Urgence Pédiatrique, CHU Ste Justine, Université de Montréal, Montréal, QC

Introduction: Resources available to manage children's pain in the pediatric emergency department (PED) may be sub-optimal. Objectives: (a) To evaluate currently available resources for pediatric pain management in Canadian PEDs and (b) To evaluate which currently unavailable resources were felt to be most amenable to rapid implementation. Methods: This cross sectional survey evaluated pain and anxiety management policies and procedures used in PEDs across Canada. All attendees of the 2012 Pediatric Emergency Research Canada (PERC) annual conference ( $>50$ pediatric emergency physicians) were asked to provide a comprehensive list of pain and anxiety management interventions available at their institution. This information was then used as the basis for creation for this survey, along with expert opinion and literature review. The study tool was pilot tested for face and content validity, and data were collected via an electronic survey. The PERC site representative for each pediatric hospital across Canada was contacted to identify the most appropriate administrator to complete the survey. Results: We had an $80 \%(12 / 15)$ response rate. Most settings (58\%) did not have access to a child life specialist; $17 \%$ thought it could be easily implemented in their centre. The most frequently used distraction method was TV/video recordings (83\%). Fifty-eight percent of the centre offered the parental lap/sitting position for IV insertion; another 33\% felt that this could be easily implemented in their center. Local anesthetic use before IV insertion was employed by $83 \%$; all remaining centres reported that this could be easily organised in their ED. Most settings used nurse-initiated protocols for local anesthetic $(67 \%)$, acetaminophen (100\%), ibuprofen (100\%) and opioids (39\%). Intranasal fentanyl was used in $58 \%$ of the centres; another $33 \%$ of centres felt it could be easily implemented. Only $36 \%$ of centres used nitrous oxide; all remaining centres felt it would be difficult to use. Biers blocks were minimally used (20\%), and were perceived as difficult to implement by $50 \%$. Conclusion: There is a wide variability in resources available at the different PEDs across Canada. We have identified some known, effective techniques for pain management (parental lap/sitting, local anesthetic for IV insertion, intranasal fentanyl) that were felt to be easy to implement. By implementing these changes first, clinicians and administrators may find greater success in addressing current deficits in children's pain management in the PED. Keywords: pain management, pediatric emergency medicine, PERC study

\section{OP34}

Pediatric pain management in the emergency department: the triage nurses' perspective

J. Kircher, MD, D. Thomas, MD, A. Plint, MD, E. Fitzpatrick, RN, A. Newton, PhD, R.J. Rosychuk, PhD, S. Ali, MDCM, University of Alberta, Edmonton, AB

Introduction: Understanding triage nurses' perspectives of pediatric pain management is essential to effective initiation of pain care for 
children in the emergency department (ED). This study describes triage nurse knowledge and perceived effectiveness of pain-related treatment protocols as well as barriers to and attitudes towards their implementation. Methods: A survey was administered to all triage nurses at three Canadian pediatric EDs - one general ED with both pediatric and adult triage, and two EDs with pediatric-only triage. A novel, paper-based survey tool was created and distributed from December 2011-January 2012, using modified Dillman methodology. Results: The response rate was $81 \%(126 / 156)$. The mean respondent age was 40 years (SD 9.3 ) with 8.6 mean years of triage nursing experience (SD 7.7). The three sites differed with the amount of pediatric pain management training $(\mathrm{p}=0.006) ; 14 \%(28 / 126)$ of respondents had experience with triage pain protocols. Nurses' perception of adequacy of triage pain treatment varied with the nurses from the general triage ED rating their site less favourably than those from the pediatric-only triage EDs $(p<0.001)$; however, perception of overall ED pain treatment did not differ between sites $(\mathrm{p}=0.88)$. General triage nurses reported a longer maximum acceptable delay between triage and administration of analgesia than pediatric-only nurses for patients with mild, moderate, and severe pain $(\mathrm{p}<0.002$ for all). Using a $100 \mathrm{~mm}$ visual analog scale (VAS, $100=$ 'very comfortable' and $0=$ 'not at all comfortable'), most nurses rated comfort with a protocol involving administration of ibuprofen $(97 \mathrm{~mm}, \mathrm{IQR} 93,100)$ or acetaminophen $(97 \mathrm{~mm}, \mathrm{IQR} 92$, $99)$ higher than for oral morphine $(67 \mathrm{~mm}$, IQR 35,94$)$ or oral oxycodone $(57 \mathrm{~mm}$, IQR 15,81$)$. The top three reported barriers to triageinitiated pain protocols were monitoring capability, time, and access to medications, while the top three facilitators were perceived personal comfort level, physicians, and other nurses. Willingness to implement a triage-initiated pain protocol was rated as $81 \mathrm{~mm}$ (IQR 71-96, $\mathrm{p}=0.54$ ), using a VAS where $100=$ 'very willing' and $0=$ 'not at all willing'. Conclusion: Triage nurses express a willingness to implement triage-initiated pain protocols for children in the ED, but differences in comfort and experience exist between pediatric-only and general triage systems. More pain management and protocol-related education may be needed to support general triage nurses.

Keywords: pediatric emergency, pain, triage

\section{OP35}

The epidural blood patch is an under-utilized treatment for postdural puncture headache in the emergency department

A. Pat, BSc, Y.S. Liu, BSc, N. Brown, MD, M. Ng, MD, S. Pytka, MD, A. McRae, MD, E. Lang, MD, College of Medicine, University of Saskatchewan, Saskatoon, SK

Introduction: Post-dural puncture headache (PDPH) is a complication of diagnostic lumbar puncture, spinal or epidural anesthesia. There is evidence that early treatment with the epidural blood patch (EBP) significantly reduces patient discomfort and prevents further morbidity. The objective of this multicentre study is to describe EBP usage in the emergency department (ED) setting. Methods: We performed a tworeviewer, retrospective chart analysis of patients with PDPH in four Calgary EDs. Patients were identified using visits from 2008 to 2012 with an ICD-10 code (G97.1) corresponding to PDPH. Headache presentations were classified based on the duration between dural puncture and physician assessment as early ( $<2$ days), or late ( $\geq 2$ days). The primary outcome was the proportion of all visits receiving an anesthesia consultation (AnCon). Secondary outcomes were the proportions of AnCon and completed EBPs for various duration of headache and the overall percentage of success for completed EBPs. Success was defined as resolution of the headache or a reduction in symptoms before discharge. Proportions were compared using Pearson's chi-squared statistics. Results: There were 249 visits by 196 patients with PDPH included in the study. Of these visits, 58.6\% (95\% CI 52.4-64.6\%) received an AnCon. The proportion of patients receiving an AnCon for early and late presentations were 37.0\% $(17 / 46)$ and $63.5 \%(129 / 203)$, respectively $(\mathrm{p}<.001$ for difference). The proportion of visits receiving an EBP for early and late presentations were $21.7 \%(10 / 46)$ and $36.9 \%(75 / 203)$, respectively $(\mathrm{p}<.05)$. A total of 85 EBPs were completed, of which $77.6 \%$ (95\% CI $67.7-$ $85.2 \%$ ) were confirmed successful. Conclusion: Emergency physicians and anesthetists in this cohort may be under-utilizing the EBP for $\mathrm{PDPH}$, in particular for headache less than two days. ED strategies to encourage prompt use of the EBP are recommended.

Keywords: post-dural puncture headache, epidural blood patch, specialist consultation

\section{OP36}

Do urine cultures in the emergency department change management of young women with symptoms of lower urinary tract infection? D.D. Phillips, MD, S.L. McLeod, MSc, M. Klingel, MSc, J. Dreyer, $\mathrm{MD}$, University of Western Ontario, London, ON

Introduction: Urinary tract infections (UTI) are one of the most commonly encountered infections in the emergency department (ED). With the rise of bacterial organisms resistant to modern antibiotics, some practitioners may order a urine culture in uncomplicated UTI despite the fact that guidelines clearly state this expensive investigation is not required. The objective of this study was to determine how many young (18-39 years) women presenting to the ED with symptoms of an uncomplicated UTI had a urine culture performed and if the urine culture results changed management. Methods: This was a retrospective medical record review of women aged 18-39 presenting at one of two tertiary care EDs with a discharge diagnosis of cystitis or UTI during the study period of May 2011-May 2012. Women who had fever, pregnancy, diabetes or cancer, were immunocompromised, were taking steroids, or had a UTI in the previous 90 days were excluded. A random sample of patients were then examined for hospital length of stay (LOS), urinalysis results, culture performance and results, antibiotics chosen, resistant organisms and a subsequent change in antibiotics or unplanned return ED visit. Results: Of the 182 charts reviewed, 120 were included in the analysis. Mean (SD) age was $26.3(6.1)$ years and median (IQR) LOS was $2.5(1.6,4.0)$ hours. $119(99.2 \%)$ patients had a urinalysis, of which $112(94.1 \%)$ had positive leukocyte esterase and $22(18.5 \%)$ had positive nitrites. $118(98.3 \%)$ patients received antibiotics $(52.5 \%$ Septra, $20.3 \%$ Norfloxacin, $16.1 \%$ Nitrofurantoin, $11.0 \%$ other). 58 patients $(48.3 \%)$ had urine cultures performed, of which 5 (8.6\%) grew resistant organisms, and $2(3.4 \%)$ received new prescriptions. There were 3 unplanned return ED visits within 7 days $(2.5 \%)$. Conclusion: The results of this study suggest that routine use of urine cultures in uncomplicated UTI in young healthy women is unnecessary and does not change management in $96.6 \%$ of patients. These findings suggest the need to educate ED staff about current guidelines for ordering urine cultures in this patient population in order to reduce unnecessary laboratory utilization.

Keywords: urinary tract infection, cystitis, culture

\section{OP37}

Improving quality measures of ED sepsis care through staff education, feedback, and use of a standardized protocol

S. Taylor, MD, M. McGowan, MHK, D. MacKinnon, MD, C. Hayes, MD, MEd, L. Barratt, MSc, K. Gaunt, MSc, E. Nagatu, BSc, S.H. Gray, MD, University of Toronto, Toronto, ON

Introduction: Early recognition and treatment of sepsis reduces morbidity and mortality. The objective was to assess change in quality measures of ED sepsis care (time-to-first-antibiotics, serial lactate measurement, time-to-3L fluid resuscitation and ED LOS) following introduction of a sepsis protocol with education and ongoing quantita- 
tive feedback to ED staff. Methods: A three-month retrospective chart review of all ED patients admitted to ICU and Step-Up with pneumonia, sepsis or urosepsis was conducted pre- and post-intervention. Patients from September to December 2012 were compared to the same months in 2013. Clinical, demographic and administrative data was extracted and $10 \%$ independently reviewed for inter-rater reliability. Outcomes were time-based from triage and in-hospital mortality. Results: 74 cases met inclusion criteria $(2012 n=31 ; 2013 n=43)$. From 2012 to 2013, we noted a modest improvement in time-to-first-antibiotics (mean, SD: 3:02 $\pm 2: 07$ vs 2:41 $\pm 0: 51 \mathrm{hr}$ ), ordering of antibiotics ( $84 \%$ vs $100 \%)$, time-to-lactate (1:23 $\pm 1: 15$ vs $1: 15 \pm 1: 06 \mathrm{hr}$ ), ordering of lactate by Emergency Physicians ( 71 vs $81 \%$ ), and ED LOS (10:51 $\pm 6: 29$ vs 10:03 $\pm 6: 18 \mathrm{hr}$ ). Several quality indicators did not improve including serial lactate measurement ( $36 \%$ vs $14 \%$ ) and timeto-3L fluid resuscitation ( $4: 18 \pm 2: 42$ vs $4: 20 \pm 2: 49 \mathrm{hr})$. Conclusion: Introduction of a standardized sepsis protocol paired with education and regular quantitative feedback to ED staff improved some quality measures of ED sepsis care (time-to-first-antibiotics, lactate ordering), but failed to impact others (serial lactate measurement, time-to-3L fluid resuscitation) despite being built into the sepsis protocol. Opportunities exist to further improve ED sepsis care and reduce mortality with ongoing feedback and encouragement of ED staff to adhere to the protocol.

Keywords: sepsis, quality indicator, quality improvement

\section{OP38}

Prospective validation of the Ottawa Heart Failure Risk Scale I.G. Stiell, MD, MSc, C.M. Clement, S. Aaron, MD, B. Borgundvaag, $\mathrm{PhD}$ Md, R.J. Brison, MD, L.A. Calder, MD, MSc, A. Forster, MD, A. McRae, MD, J.J. Perry, MD, MSc, B.H. Rowe, MD, MSc, G. Wells, $\mathrm{PhD}$, Ottawa Hospital Research Institute, Ottawa, ON

Introduction: The Ottawa Heart Failure Risk Scale (OHFRS) was previously derived to identify ED HF patients at high risk of serious adverse events (SAE) and consists of 10 criteria from history, examination, and bedside tests. We sought to prospectively validate the performance of OHFRS Methods: We conducted a prospective cohort study and enrolled consecutive adults with acute HF in 6 tertiary care hospital EDs. Patients were evaluated for the 10 OHFRS criteria and then followed for 30 days. The primary outcome, SAE, was defined as any of: death within 30 days, admission to monitored unit, intubation, non-invasive ventilation, myocardial infarction, or relapse back to the ED within 14 days followed by hospital admission. Data analysis included calculation of sensitivity and specificity. Results: We enrolled 867 patients with mean age 77.6 years, male $52.1 \%$, and admitted $58.5 \%$. SAEs occurred in $144(16.6 \%)$ cases $(21.1 \%$ in those admitted and $10.3 \%$ in those discharged from the ED): death 25 , NIV/intubation 26, MI 19, monitored unit 53, major procedure 30, relapse with admission 37 . Calculating SAE sensitivity and specificity and admission rates, we can compare current practice $(74.3 \%, 44.7 \%$, $58.5 \%$ ) to the OHFRS score $>=2$ points without NT-ProBNP values $[\mathrm{N}=867](70.6 \%, 54.8 \%, 49.4 \%)$ and to the OHFRS score $>=2$ points with NT-ProBNP [N=492] $(80.0 \%, 40.7 \%, 64.2 \%)$. Conclusion: OFHRS performed well compared to current practice and was more sensitive with NT-ProBNP values than without. This scale should help standardize disposition practices, diminishing both unnecessary admissions and unsafe discharge decisions for ED HF patients.

Keywords: heart failure, risk scale, decision rules

\section{OP39}

Potentially missed opportunities to diagnose ACS: demographic and clinical features of patients seen and discharged from an emergency department within 7 days of hospitalization for ACS
E. Lang, MD, M. Traboulsi, MD, R. Welsh, MD, L. Morrin, MBA, F. Stephenson, $\mathrm{PhD}$, University of Calgary, Calgary, $\mathrm{AB}$

Introduction: Identifying patients with acute coronary syndromes (ACS) from the myriad of patients with both typical and atypical presentations of possible ACS is a challenge for emergency department (ED) providers. Missed ACS occurs when precursors or mimics of ACS are evaluated in the ED and potentially misdiagnosed and constitutes an important quality of care indicator. We undertook an epidemiologic analysis of missed ACS to highlight unique clinical and environmental predictors. Methods: We reviewed administrative data for unplanned ACS admission over a 36-month period (2010-2012) in Alberta. Records from Alberta residents were traced to determine potentially related ED visits in 3 and 7 days prior to ACS admission. Primary outcome was the percentage of patients who recently presented for ED care. Secondary analyses compared those with and without recent ED visits. Student's t test and chi square test were used for comparisons. Results: There were 18,216 episodes of care for ACS (admitting ICD 10 Dx) over the study period; 1321 patients $(7.25 \%)$ presented to an ED 7 days prior and $4.15 \%$ presented 3 days prior to ACS hospitalization. The most common ICD 10 diagnoses at the first ED visit were pain in throat and chest $(23.5 \%)$, and angina pectoris $(9.1 \%)$. Although 221 different diagnoses were recorded, COPD (3.2\%) and abdominal pain $(3.0 \%)$ were among the most common .There were also no differences seen between regional, urban or rural centers for previous ED visit rates. Potentially missed ACS patients tended to be older; mean age of 67.7 vs $66.1 \mathrm{yrs}$, had prolonged inpatient LOS; mean 7.4 versus 6.1 days and demonstrated higher inpatient mortality; $6.2 \%$ vs $4.3 \%$; all with $\mathrm{p}<0.001$ Conclusion: Patients with ED visits in the 7 days prior to admission for ACS represent a high risk group characterized by increased disease severity. Efforts to reduce the rate of missed ACS and further evaluate the management of these patients as a quality indicator is warranted.

Keywords: acute coronary syndrome, misdiagnosis, population studies

\section{LO01}

The edus 2 workout: a stepwise approach to learning critical care emergency ultrasound

P.A. Olszynski, MD, University of Saskatchewan, Saskatoon, SK

Innovation concept: The edus 2 workout offers trainees an interactive way to apply their developing knowledge of several critical care conditions and their associated emergency ultrasound findings. The "workout" can take place both inside and outside the simulation environment, but preferably during small group learning sessions. Through the use of the edus 2 simulator and a newly designed scanning shirt (a t-shirt embedded with multiple velcro-held scan tags embedded at key anatomical landmarks), trainees cane now attempt combining the appropriate series of scans for a given indication. This invention may help emergency medicine trainees: 1) incorporate the relevant scans for a given indication 2) review key positive findings on recordings from real patients 3) develop a consistent approach to scanning protocols such as EFAST. Methods: The edus2 "workout" makes use of a previously invented emergency ultrasound simulator, the edus2 (www.edus2.com). The workout t-shirt consist of over 25 secured velcro tabs placed at appropriate anatomic landmarks. Facilitators can have one trainee wear the workout shirt and then select the appropriate clips for the other trainees to scan by placing the tags at the relevant locations. For example, a facilitator could program the shirt for cardiogenic shock by placing the following tags at their appropriate landmarks: B-Lines at lung fields, severely hypokinetic LV at cardiac views, full IVC in subxiphoid view, etc... After trainees complete the appropriate scans and demonstrates an understanding of the findings, the group can then move on to other indications. Curriculum, tool, or material: Instructions for mak- 
ing the edus 2 workout shirt are available at: www.edus2.com. Conclusion: The concept of layered learning is not new to medical training. Its theoretical origins are found in Vygotsky's Zone of Proximal Development. Expertise (defined as the ability to complete a task independently) is gained through careful guidance of trainees through their respective Zone Of Proximal Development (ZPD). The ZPD is defined as the distance between the actual developmental level and the level of potential development. The edus 2 workout allows for tailored trainee development and may avoid factors that are be detrimental to learning (excessive cognitive load, performance anxiety and safety concerns). This can be achieved through the deliberate use of the edus 2 workout in a progressively challenging fashion leading to mastery.

Keywords: innovations in EM education, emergency ultrasound, simulation

\section{LO02}

Mass gathering medical care in a motorsports event-based collaborative training program

J.M. Goodloe, MD, A.O. Arthur, PharmD, R. Genzel, MD, B.D. Burns, DO, S.H. Thomas, MD, MPH, Department of Emergency Medicine, University of Oklahoma School of Community Medicine, Tulsa, OK

Innovation concept: Emergency physicians are increasingly providing medical oversight and care at mass gathering events. The pre-event planning, dynamics, and all-hazards considerations of mass gatherings differ substantially from traditional EMS and emergency medicine practices. Focused mass gathering medical care training for emergency medicine resident physicians and EMS fellows is integral to success in their future medical oversight of such events. Methods: A multi-disciplinary faculty, comprised of emergency physicians and nurses, EMS professionals, active duty special operations military operators, law enforcement officers, firefighters, and meteorologists deliver a threeday curriculum in mass gathering medicine surrounding NASCAR and IndyCar racing events held at the Texas Motor Speedway. The curriculum includes didactics of emergency medical care planning for crowds in excess of 175,000 , with many camping on-site up to a week. Considerations of inclement weather, terrorist events, accidental chemical exposures, and substance abuses are detailed in addition to the variety of emergency medical conditions expected in any large crowd. The curriculum is taught real-time during active events at the Texas Motor Speedway, one of the largest sporting event venues in the world. Practical exercises include responding with EMS crews and working with nurses and physicians in the track hospitals, specialties including emergency medicine, general surgery, and orthopedics. On-site capabilities successfully utilized include advanced airway management, chest tube thoracostomies, plain film radiology, and intravenous thrombolytics for acute myocardial infarcts. Ground and rotor wing EMS capabilities are continuously present. Curriculum, tool, or material: Multiple physicians have been successfully trained in this collaborative program. Pre-event and post-event interviews indicate substantial didactic and clinical knowledge acquisition through the course. Consistently accurate discussion points with student physicians reveal appropriate understanding of the need for multi-disciplinary planning for the medical care to be provided at such events and for estimating likely illness and injury to be encountered. Conclusion: A multi-disciplinary faculty of medical and public safety professionals, led by emergency physicians, can translate the didactic and clinical knowledge of mass gathering medical care to emergency medicine residents and EMS fellows.

Keywords: innovations in EM education, mass gathering medicine, out-of-hospital emergency medical care

LO03

A novel program to improve patient safety by integrating peer review into the emergency medicine residency curriculum
R.J. Strayer, MD, B.D. Shy, MD, P.W. Shearer, MD, R. Kedia, MD, R.J. Patel, MD, K. Shah, MD, Mount Sinai School of Medicine, New York, NY

Innovation concept: Central to Quality Improvement programs is case-based Peer Review, which facilitates compliance with performance measure regulations and enables outcomes-based feedback to caregivers. At our institution, we integrated all 60 residents in our 4year program into Clinical Quality Review (CQR) as a continuous, core component of the curriculum. Methods: We identified $13 \mathrm{CQR}$ case groups with high learning value such as ED mortalities, 72 hour returns, and intubations; each assigned a rotating team of 2-6 residents. We developed standardized chart abstraction methods and reporting guides for each team. Monthly, residents enter the clinical data they have abstracted into structured spreadsheets tailored to departmental quality goals; these spreadsheets also serve as resident case review portfolios. Each team presents their findings at the monthly departmental CQR meeting, where residents help identify systems issues and learning points to improve future care. Curriculum, tool, or material: We successfully integrated Emergency Medicine residents into our ED QI/PS program. From January through December 2012, 60 residents reviewed 4,458 charts at our two hospital sites. Of these, 194 cases were flagged for review, which led to variety of provider and systemstargeted corrective actions and teaching points. In a survey of all 60 residents, $85 \%$ reported that $\mathrm{CQR}$ made them a better clinician and $77 \%$ reported that the quality of care improved due to resident participation in CQR. Conclusion: We believe this is the first comprehensive, resident-driven, longitudinal peer review program of this scale that has integrated residents throughout their residency into departmental QI/PS functions. As improving the quality of care and reducing medical error has become a focus of healthcare, and as residencies are tasked with training residents in quality assurance, other institutions may find value in a similar program.

Keywords: innovations in EM education, quality assurance, peer review

\section{LO04}

Peripheral vertigo discharged from the emergency department: examining outcomes in the province of Ontario

K. Grewal, MD, P.C. Austin, PhD, H. Lu, PhD, M. Kapral, MD, MSc, G. Kulkarni, MD, PhD, C.L. Atzema, MD MSc, University of Toronto, Toronto, ON

Introduction: Dizziness is a common complaint in emergency departments (EDs). The purpose of this study was to confirm the safety of current practice for the management of peripheral vertigo in Canada, by examining outcomes of patients with peripheral vertigo who are discharged from EDs across Ontario, Canada. Methods: Using an administrative database that contains abstracted data on all ED visits in the province of Ontario, patients 18 and older with a primary ED diagnosis of peripheral vertigo (ICD-10 codes H81.1-81.3) who were seen in an Ontario ED between March 2006 and April 2011 were identified. Using propensity score matching on 32 demographic and comorbid variables, discharged patients were matched to discharged ED patients with a primary diagnosis of renal colic or urinary tract infection (UTI). Outcomes included 30-, 90-, and 365-day mortality and strokes. Results: There were 34,872 and 39,933 matched colic and UTI patients, respectively, with a mean age of 52.8 and 56.3, and a history of stroke in $0.6 \%$ and $0.9 \%$. Among the UTI-matched vertigo cohort, $385(1.0 \%)$ died and $167(0.4 \%)$ had a stroke within a year of discharge; among colic-matched vertigo cohort, $252(0.7 \%)$ died and 124 $(0.4 \%)$ had a stroke by 365 days. Discharged peripheral vertigo patients had lower 30-, 90-, and 365-day mortality than matched colic patients: RR 0.37 (95\% CI: 0.20-0.66), 0.52 (95\% CI: 0.38-0.72), and 
0.75 (95\% CI: 0.64-0.88), respectively. Mortality was also lower compared to matched UTI patients: RR 0.09 (95\% CI: 0.05-0.15), 0.17 (95\% CI: $0.13-0.21), 0.30$ (95\% CI: 0.27-0.33), respectively. Peripheral vertigo patients had significantly higher risk of stroke compared to the matched cohorts: vertigo vs. colic patients RR 9.29 (95\% CI: 4.2620.25), 6.07 (95\% CI: 3.45-10.69), 2.53 (95\% CI: 1.82-3.52), and RR for vertigo vs. UTI patients 1.95 (95\% CI: 1.31-2.89), 2.02 (95\% CI: $1.43-2.85$ ), 1.10 (95\% CI: 0.88-1.37), at 30-, 90-, and 365-days, respectively. In a post-hoc analysis examining mortality, colic and UTI patients were more likely to undergo surgery subsequent to the ED visit compared to patients with peripheral vertigo. Conclusion: While patients discharged from the ED with peripheral vertigo had lower short and long-term mortality compared to matched counterparts, they had significantly higher risk of stroke. The increased rate of stroke among peripheral vertigo patients is concerning for missed central causes of vertigo; however, the actual numbers were small.

Keywords: peripheral vertigo, stroke

\section{LO05}

Patient and caregiver health literacy in stroke: a systematic review G. Goel, BSc, I. Poureslami, PhD, R. Stenstrom, MD, PhD, D.R. Harris, $\mathrm{MD}, \mathrm{MHSc}$, University of Toronto, Toronto, $\mathrm{ON}$

Introduction: Cerebrovascular disease (CVD) is the third leading cause of death in Canada and the leading cause of adult disability. Patient-centered care and self-management of CVD, and chronic diseases in general, require patients and caregivers to have a functioning level of health literacy. Within the stroke community, It is not well understood how health literacy impacts patient outcomes, nor which interventions exist to measure and improve stroke health literacy. Our objective was to perform a systematic review to classify the components of health literacy as it pertains to stroke, and to assist in the development of a stroke health literacy measurement and intervention tool. Methods: This was a systematic review of the published literature. Search terms included 'health literacy,' 'stroke,' 'literacy', and five related components of health literacy (e.g. accessing, understanding, evaluating, communicating, and using health information). We searched MEDLINE, EMBASE, CINAHL, Cochrane Library, Informit Health Collection, ERIC, Sociological Abstracts, Social Science Abstracts, PsycINFO, and Health and Psychosocial Instruments. We limited our search published between Jan. 1, 1980 to Nov 1, 2013 in English. Manual hand-searching was performed; grey literature was also explored. Inclusion was determined by three independent reviewers; data was extracted through a standardized form. Quality was assessed by two independent reviewers. Data from included papers was then extracted and entered into a display matrix, and grouped into themes. Results: The search strategy yielded 112 potentially relevant articles. Of those, 25 were selected for inclusion in the review. To evaluate relevance of the selected interventions for stroke patients, we selected studies that applied health literacy as an intervention (or part of a combined intervention) as part of the patient's self-management. Doing so, five major themes were identified from the literature: (1) formatting and content of educational materials; (2) appropriateness of current educational materials; (3) effectiveness of current educational materials; (4) measurement of health literacy in stroke patients; and (5) the effect of health literacy on outcomes. There is a paucity of tools designed to measure all components of health literacy, especially in relation to stroke care. As expected, lower health literacy is associated with poorer outcomes after stroke: higher health literacy is significantly associated with fewer ED visits, fewer hospitalizations, higher self-reported health status, higher disease knowledge, and more preventive care. Conclusion: From this systematic review, we identified that health literacy as it pertains to stroke self-management is an undefined and significant burden. Health literacy had a direct rather than indirect effects on health outcomes for stroke survivors. A stroke health literacy measurement tool would be beneficial to assist in the design and implementation of health literacy interventions.

Keywords: stroke, health literacy, systematic review

\section{LO06}

Rural versus urban discrepancies in 30-day in-hospital mortality following stroke: CIHI and the Canadian rural emergency medicine project

R. Fleet, MD, PhD, S. Bussières, PhD, F. Tounkara, MSc, G. Dupuis, PhD, P.M. Archambault, MSc, MD, J. Plant, MD, J. Poitras, MD, Université Laval and CHAU Hôtel Lieu de Lévis, QC

Introduction: Twenty percent of Canadians live rurally and providing quality emergency care to this population in the context of limited resources is a challenge. For ex. few rural EDs have 24/7 local access to a CT scanner. The Heart and stroke foundation of Canada recommends early identification and treatment of suspected stroke patients with a critical decision point being the performance and interpretation of a CT scanner within 45 minutes of arrival in the ED. With most rural EDs located outside time frames for recommended stroke care, we hypothesized that mortality following stroke would be greater in rural versus urban tertiary hospitals. Methods: We used data from the Canadian Hospital Reporting Project tool available on the Canadian Institute for Health Information (CIHI) website. Data on adjusted 30day in hospital mortality following stroke was cumputed from 2007 to 2011 for all acute care hospitals in Canada excluding Quebec. Only rates with denominators $>4$ are reported. We selected hospitals located in rural small towns providing $24 / 7$ emergency physician coverage with hospitalization beds. Urban tertiary centers were principally academic, designated Level 1 and 2 trauma centers. We compared provincial mean 30-day adjusted stroke mortality rates between rural and urban hospitals. Also, we compared hospital characteristics between rural reporting and non-reporting centers. Results: There were 290 rural and 24 urban hospitals meeting our criteria. In total, $10 \%$ of rural hospitals had local 24/7 access to a CT scanner and $19 \%$ had in-hospital ICU, in contrast to $100 \%$ of urban centers (a criteria for trauma center designation). CIHI data was available for $44 \%$ of those hospitals we considered to be rural and $96 \%$ of urban. For every province and every year, the 30-day adjusted stroke mortality was significantly higher in rural than urban hospitals. Nationally, rural versus urban hospital mean 30-day stroke mortality (rate per 100) was for 2007:21.01 (CI95\%: 20.66 - 21.36) and 16.92 (CI95\%: 16.28 - 17.56) ); 2008 : 17.78 (CI95\%: $17.01-18.54)$ and 16.18 (CI95\%: $15.05-17.30) ; 2009$ : 19.42 (CI95\%: 18.74 - 20.09) and 16.03 (CI95\%: 14.98 - 17.07); 2010: 18.01 (CI95\%: 16.68 - 19.34) and 14.95 (CI95\%: 13.96 - 15.95); 2011: 20.17 (CI95\%: $19.28-21.06)$ and 14.96 (CI95\%: $13.83-16.09)$. Despite unstable values in certain rural centers, the stability of combined yearly rural data and, with few exceptions, non overlapping CIs suggest strong trends in findings. Furthermore, there was no tendency in the rural versus urban stroke mortality gap - reduction between 2007 and 2011. The mortality was also higher in rural centers without versus with CT scanners (22.1 versus 17.1). Finally, reporting rural centers had significantly more ED visits and were more likely to have a CT scanner, and ICU than non-CIHI reporting centers $(\mathrm{p}<0.01)$. Conclusion: Between 2007-2011, overall 30-day adjusted stroke mortality was, with few exceptions, higher in rural than urban tertiary hospitals in Canada. Results must be interpreted in the context of limited CIHI website-reported data for rural hospitals and absence of data from Quebec. While the rural and urban gap in access to emergency care resources is increasingly acknowledged, this is the first report on disparities in actual patient outcomes. Future studies are required to examine reasons for outcomes disparities.

Keywords: health indicators, rural, stroke 
$\mathrm{LO} 07$

The McMaster Modular Assessment Program (McMAP): the junior emergency medicine competency pilot project

T.M. Chan, MD, J. Sherbino, BSc, MD, MEd, I. Preyra, MD, McMaster University, Hamilton, ON

Innovation concept: With CanMEDS 2015, a shift in residency training from a time-based system to a competency-based framework is anticipated. Competency-based medical education (CBME) requires robust workplace-based assessment (WBA) of learner performance. In expectation of this change, the McMaster University Emergency Medicine program developed a CBME-based assessment system. The McMaster Modular assessment Program (McMAP) uses a cloud-based system; this allows for point-of-care access via smart phones or hospital-based computer terminals. Methods: A modular assessment program was created with 52 unique WBAs that mapped to the CanMEDS Roles. We collaborated with teams from two other universities and reviewers from anotherfour4 universities, informed by the best available literature. After a year, our pilot program has resulted in a shift in local educational practice. McMAP ensures repeated observation of core emergency medicine tasks by trainees. Each WBA template provides a just-in-time faculty development resource to support a shared mental model between faculty observers. Curriculum, tool, or material: McMAP uses a model of graded competencies with second year residents completing more sophisticated WBAs than the first year residents. Over the course of our pilot year, we collected over 2000 pieces of quantitative data and 1500 pieces of qualitative commentary on 14 residents. On average, the WBAs require 5-10 minutes to complete. The required narrative feedback provides learners with specific, tailored formative feedback. Summative feedback is created by merging data from all of the WBAs and requiring the rotation supervisor to analyze this data for common themes. Conclusion: In the shift to CBME, robust assessment will be imperative. Graded and work-based assessments are important for linking observed behaviour to conceptual frameworks. Data from multiple sources about multiple competencies is key. McMAP is a feasible example of a CBME assessment system for the Emergency Medicine environment.

Keywords: innovations in EM education, assessment, competencybased medical education

\section{LO08}

An innovative online curriculum for accredited continuing medical education in geriatric emergency medicine: www.geri-EM.com D. Melady, MD, University of Toronto, Toronto, ON

Innovation concept: This project used material originally developed for resident education, expanded and re-purposed it for the practicing $\mathrm{EP}$, and made it available in an online format. The content is unique in addressing the EM competencies for providing care to older patients. The method is innovative in providing EM-specific online learning, accredited for CME in Canada. Methods: We reproduced, in an asynchronous online environment, the experience and benefits of selfdirected interactive learning associated with the small group experience. We developed a highly interactive website: immediate feedback to quiz material, opportunities for self-assessment of learning needs, video presentation of challenging cases using standardized patients, opportunities to interact with other site users and a moderator, chat and blog features, links to other learning resources (readings), and downloadable clinical tools. Curriculum, tool, or material: The tool is a six-module educational website that addresses the core competencies of ED care of older people. The website allows the user to identify personally relevant needs. Each module, approximately one hour in length, promotes learning through immediate feedback to knowledge checks and through didactic material presented around videos of simu- lated patient encounters. There is an opportunity for users to interact asynchronously - with other users by seeing and responding to others' comments and to receive expert feedback. Discussion takes place through a comment board. We worked extensively with both the Royal College and the College of FP to ensure the tool met the highest standards for interactive self-directed learning (SAP and MainPro-C.) Conclusion: This project builds on work by ACEP and SAEM to delineate specific competencies in Geriatric Emergency Medicine. Its innovations are two-fold. It addresses a topic - ED care of the older patient - for which there are few or no other learning resources. And it presents the material in an innovative online format. It is the first online resource accredited for CME for EPs by both Canadian colleges. In six months it has approximately a thousand interdisciplinary users and nearly 500 modules completed for credit.

Keywords: innovations in EM education, geriatric emergency medicine, continuing medical education

\section{LO09}

Development and implementation of core competencies for an emergency medicine point-of-care ultrasound rotation using the CanMEDS framework

J. Chenkin, MD, University of Toronto, Toronto, ON

Innovation concept: In the past several years, the Royal College of Physicians and Surgeons of Canada has mandated point-of-care ultrasound (POCUS) to be a core skill for graduating emergency medicine residents. To ensure that our residents were meeting this requirement, as well as to adjust for increasing demands for more advanced ultrasound training, we have implemented a novel POCUS rotation to the emergency medicine residency training program at the University of Toronto. This new rotation presented a unique opportunity to develop goals and objectives tailored to match competency-based outcomes. Methods: Using the CanMEDS framework, local experts in the field of resident education and POCUS developed goals and objectives for each of the core competencies. This list was revised until there was agreement. Further revisions were made after pilot-testing during several phase-in resident POCUS electives. Curriculum, tool, or material: The POCUS core competencies were implemented using a multifaceted approach. During their hands-on training, residents received daily feedback using clinical encounter cards that evaluated aspects of all seven CanMEDS roles. Weekly reflective assignments were used to evaluate specific objectives that were seldom encountered during hands-on training. The manager role was evaluated through the use of a quality assurance assessment tool. The scholar role was evaluated through the use of an ultrasound journal club. A summative standardized examination was used to ensure that the medical expert objectives were met. Conclusion: Using the CanMEDS framework, we have developed and implemented core competencies for our novel emergency medicine POCUS rotation. The use of this framework has helped to ensure that all CanMEDS roles are being evaluated. Going forward, we hope to use this model to develop competency-based outcomes for advanced ultrasound training at the University of Toronto.

Keywords: innovations in EM education, point-of-care ultrasound, evaluation

\section{LO10}

Quebec-Fresno Shoulder Disclocation Rule: a combined decision tool

M. Emond, MD, MSc, G. Hendey, MD, Université Laval, Québec, QC

Introduction: Recently, Canadian and American clinical decision instruments have been suggested to guide selective pre-reduction radiography in patients with acute anterior shoulder dislocation. Objective: We prospectively refined a combined Quebec-Fresno clinical decision 
rule to guide pre-reduction radiography for emergency department (ED) patients with anterior shoulder dislocation. Methods: This prospective cohort derivation/refinement study took place at 2 university-affiliated EDs over a 2-year period (2010-12) and enrolled patients with acute anterior shoulder dislocation who were 16 years of age or older. We compared patients with a clinically important fracture-dislocation with those who had an uncomplicated dislocation to provide the clinical decision rule components using recursive partitioning analyses. Results: 204 patients were included in the study. Twenty-four (11.8\%) had clinically important fracture-dislocation. A clinical decision rule using 3 factors reached a sensitivity of $100 \%$ (95\% confidence interval [CI] $85.7 \%-100 \%)$, a specificity of $50 \%(95 \%$ CI $42.5 \%-57.5 \%)$ and a negative predictive value of $100 \%$ (95\% CI 96\%-100\%). Patients with an atraumatic, recurrent dislocation had no fractures. Only 3 patients younger than 35 years had a fracture, and all three had a significant mechanism, defined a priori as MVA, Assault, Sports related, or Fall $>10$ feet. Every patient with a clinically important fracture-dislocation had at least one of 3 factors: age over 35, first dislocation, or significant mechanism. Use of the combined algorithm would reduce the number of pre-reduction radiographs by $44 \%$, while missing no fractures. Conclusion: The combined Quebec-Fresno shoulder dislocation rule for patients with acute anterior shoulder dislocation could potentially reduce radiographs while missing no fractures.

Keywords: shoulder dislocation, clinical decision tool

\section{LO11}

Efficacy of anticholinergics for preventing hospitalization in adults with acute asthma

S.W. Kirkland, MSc, C. Vandenberghe, MEd, B. Voaklander, T. Nikel, R. Chetram, A. Davidson, S. Campbell, MLS, B.H. Rowe, MD, MSc, University of Alberta, Edmonton, AB

Introduction: Short-acting beta2-agonists (SABA) and short-acting anticholinergics (SAAC) are effective therapies for acute asthma; however, it is unclear whether combination therapy of SABA and SAAC is more effective than SABA alone. The objective of this study was to compare the benefit of SAAC in addition to SABA in preventing hospitalization in adults with acute asthma. Methods: Seven electronic databases, the grey literature and the Airways Register were searched. Included studies were controlled clinical trials comparing the effectiveness of SAAC and SABA to SABA treatment alone to prevent hospitalization in adults with acute asthma in the emergency department. Two independent reviewers judged the relevance, inclusion, and risk of bias of the studies. Individual and pooled statistics were calculated as relative risks (RR) with $95 \% \mathrm{CI}$ using a random effects model and heterogeneity (I2) was reported. Results: From 1,205 citations, 22 studies were included in this review. Overall, combination therapy reduced the risk of hospitalization compared to SABA alone (RR 0.73; 95\% CI: $0.59,0.90)$. Heterogeneity was low $(I 2=19 \%)$. Despite a trend for patients receiving both SAAC and SABA to experience increased side effects, such as tremor or dry mouth, no significant differences were found (RR 1.27; 95\% CI: 0.97, 1.67). Heterogeneity was low (I2 = $0 \%$ ). Conclusion: Overall, combination therapy (SAAC and SABA) was more effective in preventing hospitalization in adults with acute asthma presenting to the ED. Safety issues were minor and similar in both groups. These results should be added to asthma guidelines.

Keywords: asthma, anticholinergics, beta2-agonists

\section{LO12}

Effect of triage nurse initiated radiography using the Ottawa Ankle Rules on emergency department length of stay at a tertiary care center
W.W. Lee, MD, L. Filiatrault, MD, R.B. Abu-Laban, MD, MHSc, A. Rashidi, BSc, L. Yau, BMSc, N. Liu, BSc, Department of Emergency Medicine, University of British Columbia, Vancouver BC

Introduction: Emergency department (ED) overcrowding has prompted many initiatives to improve throughput. The Ottawa Ankle Rules (OAR) are well validated, however their use by triage nurses is poorly understood. Our objective was to determine the effect of triage nurse initiated radiographs using the OAR on ED throughput. We hypothesized OAR use would reduce median ED length of stay (LOS) by 25 minutes or more, a threshold we deemed clinically significant a priori. Methods: A randomized controlled trial was conducted from $08 / 2012$ to $08 / 2013$ at a tertiary trauma centre ED with an annual census of over 90,000 patients. Adult patients presenting within 10 days of isolated blunt ankle trauma were eligible. Participants were randomly assigned by sealed envelope to standard triage or OAR application, including radiography when indicated, by 15 explicitly trained triage nurses. Consent was waived, thus participants were blinded to the study and hypothesis. Our primary outcome was ED LOS, defined as time from arrival to discharge, orthopedic consultation, or further imaging. Secondary outcomes included triage nurse and patient satisfaction. Nurse/emergency physician (EP) OAR agreement was evaluated with the kappa statistic, and a power calculation estimated 142 patients were required. The Mann-Whitney U test was used to compare the medians between the two groups. Research ethics board approval was obtained. Results: Of 176 patients with blunt ankle injury seen by participating triage nurses during the study period, 146 were enrolled $(83.0 \%) ; 75$ in the control group and 71 in the OAR group. Baseline characteristics in the two groups were similar. The median/mean ED LOS in the control and OAR groups was 128/143 minutes and 108/115 minutes respectively (median difference 20 minutes; $p=0.003$ ). Agreement in OAR use between EPs and nurses was moderate (kappa 0.46/0.77 for foot/ankle rule components), and satisfaction of both nurses and participants was high. Conclusion: Triage nurse initiated radiography using the OAR is accepted by both nurses and patients, and leads to a statistically significant decrease in the median ED LOS at a tertiary care center, but failed to meet our predefined threshold for clinical significance. The overall impact of implementing such a process is likely site specific, and the decision to do so should include consideration of the local context

Keywords: Ottawa ankle rules, overcrowding, triage nurse

\section{LO13}

Frailty measures are useful in identifying independent seniors at risk of functional decline evaluated in emergency departments after minor trauma

M. Sirois, PhD, M. Emond, MD, MSc, J.J. Perry, MD, MSc, J.S. Lee, MD, MSc, Centre de Recherche du CHU, QC

Introduction: $70 \%$ of Canadian independent seniors consulting Emergency Departments (EDs) for minor injuries are discharged home within 48 hours. However, a cumulative incidence of $15 \%$ of functional decline has been reported up to six months post-injury in this population. An undetected pre-frail status could explain this decline. Objectives: To describe the frailty status of independent seniors consulting EDs for minor injuries and compare the capacity of two frailty measures to predict functional decline in this population. Methods: A prospective cohort study was conducted in six Canadian EDs. 1072 seniors were evaluated at the ED visit, three and six months post-visit. The "Study of Osteoporotic Fracture " (SOF) and the "Canadian Health Study of Aging » (CHSA) frailty measures were used. Functional decline was defined as a loss $\geq 2 / 28$ points on the " Older American Resources Scale » functional scale. Areas under the ROC curves were used to compare the predictive capacity of the frailty measures. 
Results: According to the « SOF » $12 \%$ of seniors were frail, $33 \%$ prefrail and 55\% robust. According to the CHSA 10\% were frail, 38\% pre-frail and $52 \%$ robust. There were $15 \%$ decliners at 3 and 6 months. Adjusted proportions of decliners were $1 \%$ in robust seniors, $14 \%$ in pre-frail and $21 \%$ in frail individuals according to the SOF. Respective proportions according to CHSA were $5 \%, 11 \%$ and $21 \%$. The «SOF» and «CHSA» ROC curves were 0.787 and 0.653 . Conclusion: As a whole, $15 \%$ of still independent seniors show functional decline up to 6 months post-injury. As measured by the SOF or CHSA, pre-frail and frail individuals are at increased risk of decline. Both measures are easily performed in EDs. The SOF has a better predictive capacity and could be included in routine assessment of injured seniors in the EDs.

Keywords: frailty, minor injury, geriatrics

LO14

Predictors of time from headache onset to computed tomography in emergency patients with suspected subarachnoid hemorrhage M. Khan, MSc, J.J. Perry, MD, MSc, M. Sivilotti, MSc, MD, University of Ottawa, Department of Emergency Medicine, Ottawa, ON

Introduction: Computed tomography (CT) has maximal sensitivity for subarachnoid hemorrhage (SAH) when performed within six hours of headache onset. This time effect is widely assumed to reflect dispersion and lysis of blood within the cerebrospinal fluid (CSF), but may also be influenced by volume and severity of SAH. The objective of this study was to assess the impact of clinical characteristics and hospital system variables on the time from headache onset to CT among alert and neurologically intact emergency department (ED) patients with suspected SAH. Methods: This was a prospective cohort study at the EDs of 11 university affiliated tertiary care teaching hospitals in Canada from November 2000 to July 2010. Subjects were neurologically intact adults with an acute, non-traumatic headache in whom CT was performed to rule out SAH. Data collected included clinical characteristics, mode of transport to the ED and triage risk assessment (Canadian Triage Acuity Scale, CTAS). Our primary outcome was time from headache onset to CT. Multivariable Cox proportional hazards modelling was performed to identify predictors of time to CT. Results: Of 2,412 ED headache patients enrolled, 194 were ultimately deemed SAH positive. In patients with $\mathrm{SAH}$, median times from headache onset to hospital registration and hospital registration to CT were 4.5 hours (IQR=21.0) and 1.9 hour ( $\mathrm{QQR}=1.65)$ respectively, compared to 9.6 hours ( $\mathrm{IQR}=43.2$ ) and 2.5 hours ( $\mathrm{IQR}=2.4$ ) among patients without SAH. In the multivariable model, variables associated with a decreased time from headache onset to CT included onset during exertion $(\mathrm{HR}=1.2,95 \% \mathrm{CI}=1.02-1.32)$, witnessed loss of consciousness $(\mathrm{HR}=1.3,95 \% \mathrm{CI}=1.03-1.60)$, increased systolic blood pressure $(\mathrm{HR}=1.04 / 10 \mathrm{mmHg}, 95 \% \mathrm{CI}=1.02-1.05)$, higher triage acuity(CTAS $1-2 \mathrm{HR}=1.9,95 \% \mathrm{CI}=1.60-2.16$; CTAS $3 \mathrm{HR}=1.5$, $95 \% \mathrm{CI}=1.30-1.67$; both relative to CTAS $4-5$ ), arrival by ambulance $(\mathrm{HR}=1.2,95 \% \mathrm{CI}=1.08-1.32)$, and worst headache of life $(\mathrm{HR}=1.5,95 \% \mathrm{CI}=1.32-1.75)$. Conclusion: Various clinical and system variables indicative of severity predict the time to CT from headache onset. By far the greatest delay is prior to hospital arrival, implying that patients present sooner when the headache is more serious. These effects may account for some of the improved sensitivity of early $\mathrm{CT}$, even in the relatively narrow spectrum of SAH patients who are otherwise alert and neurologically intact at presentation.

Keywords: subarachnoid hemorrhage, computed tomography, headache

LO15

Test characteristics of point-of-care ultrasonography for the diagnosis of acute posterior ocular pathology
M.Y. Woo, MD, N. Hecht, MBBS, B. Hurley, MD, D. Stitt, BSc, P. Pageau, MD, J. Worrall, MD, M. Reardon, MD, M. Taylor, MD, V. Thiruganasambandamoorthy, MD, MSc, University of Ottawa, Department of Emergency Medicine, Ottawa, ON

Introduction: Acute onset of flashers/floaters is a common ocular presentation. It may be caused by acute posterior ocular pathology, including retinal detachment (RD), retinal tear (RT), posterior vitreous detachment (PVD), or vitreous hemorrhage (VH). Point-of-care ultrasonography (PoCUS) performed by emergency physicians may help with the diagnosis of these pathologies. Our objective was to determine the test characteristics of PoCUS for the diagnosis of RD, RT, PVD and VH. Methods: A prospective observation study was performed on a convenience sample of patients presenting to an academic emergency general ophthalmology clinic. Adult patients with acute flashes/floaters or new visual field defects were included and those with previous RD or ocular surgery were excluded. PoCUS was performed by a blinded trained medical student and compared with a retina specialist exam. An expert PoCUS clinician reviewed all images. 6 week follow-up was completed. The primary outcome was the diagnosis of RD, RT, PVD and VH. Analyses included descriptive statistics, test characteristics, and kappa for agreement between sonographer and reviewer. Results: 62 patients were enrolled with a mean age of 60.8 years and $37.1 \%$ were female. The mean time to scan was 7.4 minutes. There was no change in diagnosis at 6 weeks. The sensitivities and specificities are: any pathology $(\mathrm{n}=60) 88.3 \%(95 \% \mathrm{CI} 86.8-89.9 \%)$ and $50 \%(95 \% \mathrm{CI}$ 2.7-97.3\%), RD (n=6) $100.0 \%(95 \%$ CI $53.9-100 \%)$ and $67.9(95 \% \mathrm{CI}$ $62.9-67.9 \%), \mathrm{RD} / \mathrm{RT}(\mathrm{n}=23) 47.8 \%(95 \% \mathrm{CI} 30.8-62.2 \%)$ and $82.1 \%$ (95\%CI 72.0-90.6\%), PVD ( $\mathrm{n}=47) 80.9 \%$ (95\% CI 74.7-88.0\%) and 33.3 (95\% CI 14-55.7\%), and VH ( $\mathrm{n}=14) 43.0 \%$ (95\% CI 21.4-58.0\%) and 93.8 (95\% CI 87.5-98.2\%) respectively. The kappas for any pathology, RD, PVD, and VH are $0.32,0.35,0.36$, and 0.1 respectively. Conclusion: PoCUS detected all RDs but has limited use for the diagnosis of RTs. PoCUS interpretation must be done at the bedside to improve kappa. Larger studies are required prior to using PoCUS for the diagnosis of acute posterior ocular pathology.

Keywords: imaging, ultrasound

\section{LO16}

Point-of-care ultrasound use over six-month training period in Rwandan district hospitals

P.C. Henwood, MD, J.S. Rempell, MD, MPH, A.S. Liteplo, MD, A.F Murray, MD, D.C. Mackenzie, MDCM, M.M. Leo, MD, S. Vaillancourt, MD, MPH, E.R. Douglass, BA, D. Dukundane, MD, S. Rulisa, MD, A.J. Dean, MD, V. Noble, MD, Brigham and Women's Hospital, Boston, MA

Introduction: Point-of-care ultrasound (POCUS) is an effective diagnostic technology in resource-limited settings. There is increasing interest in introducing ultrasound training in such environments, but few reports describing long-term follow-up and impact of a POCUS program in a resource-limited setting. We introduced a POCUS program in Rwanda, and sought to determine the number and type of ultrasounds performed, the impact of a remote quality assurance (QA) program, and the effect of POCUS on patient management. Methods: Seventeen Rwandan physicians underwent a ten-day training course in POCUS in Kigali, Rwanda. Post-course, participants tracked the ultrasounds they performed using a cloud-based storage system, recorded clinical impressions, and received periodic QA with on-site proctoring over a six-month follow-up. Remote QA to evaluate image quality was performed by five emergency ultrasound fellowship-trained clinicians. Images were graded on a scale of $0-4$. ( $0=$ no meaningful image, $2=$ adequate, $4=$ outstanding). Trainees also documented how POCUS changed clinical management. Results: Over six months, 1158 ultra- 
sounds were performed and logged by fifteen participants at eleven regional hospitals. $590(50.9 \%)$ had matched images and interpretations available for review. Abdominal ultrasound was the most frequently performed application (19.7\%), followed by liver (14.6\%), obstetrics (14.5\%), renal (12.4\%), and spleen (11\%). Across all applications, the mean score was 2.5 (SD $+/-0.11,95 \%$ confidence interval, 2.39-2.54). Ultrasound resulted in a management change in $84 \%$ of cases. Major changes in management related to medication choice $(42.4 \%)$, admission (30\%), transfer to a higher level of care $(28.1 \%)$, and performance of procedures (23.3\%). Conclusion: During this sixmonth training program in Rwanda, participants used POCUS for a range of applications. The remote QA process captured $51 \%$ of ultrasounds performed. Of scans with QA, the average score was relatively high. POCUS routinely changed clinical management. This study demonstrates the impact of POCUS in a resource-limited setting.

Keywords: ultrasound, practice patterns, medical education

\section{LO17}

Emergency physicians' ultrasound accuracy detecting intrauterine pregnancy and fetal cardiac activity

C. Varner, MD, B. Borgundvaag, PhD MD, S.M. Carver, BSc, Mount Sinai Hospital, Toronto, ON

Introduction: One in four women will experience vaginal bleeding during the first trimester of pregnancy, and since the advent of bedside emergency department (ED) ultrasound (US), patients have been given more immediate information about their pregnancy such as the presence or absence of an intrauterine pregnancy (IUP) and the presence or absence of fetal cardiac activity (FCA). Once an IUP is documented, the possibility of ectopic pregnancy is negligible, and the patient can usually be safely discharged with outpatient follow-up. The Canadian Emergency Ultrasound Society (CEUS) has rigorous credentialing and recommends physicians attain Independent Practitioner (IP) certification before performing ED US without supervision in order to prevent false interpretation of IUP. To date, no studies have evaluated the accuracy of ED physicians detecting IUP as compared to radiologists. Thus, this study seeks to evaluate the accuracy of ED physicians documenting IUP and FCA in patients presenting to the ED with vaginal bleeding in early pregnancy. Methods: We conducted a prospective, observational cohort study. All patients less than 20 weeks gestational age presenting to the ED with vaginal bleeding were enrolled. CEUSIP certified ED physicians performed transabdominal US and documented the presence or absence of IUP and FCA. These results were compared to the results of radiology-performed transvaginal US within 72 hours of ED discharge. Results: In a one month period in this ongoing study, 43 patients underwent both ED US and radiology US. ED physicians were very accurate in their bedside US assessments of documenting IUP ( $90 \%$ sensitivity and $100 \%$ specificity, $\mathrm{P}<0.001)$ as compared to the gold standard of transvaginal US performed by an experienced radiologist. ED physicians were also very accurate at identifying FCA $(83.3 \%$ sensitivity and $100 \%$ specificity, $\mathrm{p}<0.001)$. Importantly, no false positive tests documenting IUP or FCA occurred. Conclusion: Missed ectopic pregnancy is a major concern with life-threatening implications. For this reason, CEUS's training is conservative and emphasizes the importance of avoiding false positive in documenting IUP. Bedside US performed by IP-certified ED physicians are, in fact, highly reliable for detecting IUP and FCA as compared to US interpreted by radiologists, and resulted in no false positive tests for either IUP or FCA.

Keywords: ultrasound, pregnancy, ectopic

\section{LO18}

Determining factors that predict higher utilization of CT in emergency medicine
A. Kadhim-Saleh, BHSc, MSc, J. Worrall, MD, M. Gatien, MD, M. Taljaard, PhD, J.J. Perry, MD, MSc, University of Ottawa, Department of Emergency Medicine, Ottawa, ON

Introduction: High rates of computed tomography (CT) ordering may increase cost and expose patients to unnecessary radiation, contrast, and further tests. Our objective was to determine factors which influence ordering practices. Methods: A retrospective review of an imaging database at two adult teaching hospital Emergency Departments was conducted in January, March, June, and September of 2012. Departmental billing records were used to determine numbers of patients seen per emergency physician (EP). Physicians completed a paper survey to rate, on a 4-point Likert scale, 10 predictors that may influence their CT ordering, and to estimate their rate of ordering. The survey also included the following demographic data: age, sex, residency training, and years of practice. Data were analyzed using descriptive statistics; a multivariate Poisson regression analysis was used to determine factors that predicted actual ordering rate. We tested for collinearity, accounted for over-dispersion, and used backward elimination to identify statistically significant factors. Results: During the study period, 59 EPs saw 45,834 patients, and ordered 6,513 CTs, a mean ordering rate of $14.2 \%(95 \%$ CI $13.9-14.5 \%)$. The median ordering rate was $14.9 \%$ (IQR 19.9-16.9\%). Forty five EPs (76.3\%) completed the survey. In the multivariate analysis, higher ordering was associated with increasing strength of response to the following predictors: medico-legal risk ( $\mathrm{RR}=1.18,95 \%$ CI $1.03-1.21)$, risk of contrast $(\mathrm{RR}=1.14,95 \% \mathrm{CI}: 1.07-1.22)$, what colleagues would do $(\mathrm{RR}=1.09$, $95 \%$ CI: 0.99-1.19), risk of missing a diagnosis ( $\mathrm{RR}=1.08,95 \% \mathrm{CI}$ : $0.98-1.21$ ), and patient wishes ( $R R=1.07,95 \%$ CI: 0.97-1.17). Compared to our ambulatory care (Urgent) shifts, CT ordering was higher on shifts with increased patient acuity: Resuscitation (monitored area) $(\mathrm{RR}=3.02$; 95\% CI: 2.64-3.44), Observation (non-monitored stretcher area) $(\mathrm{RR}=2.42 ; 95 \% \mathrm{CI}: 2.11-2.78)$, and single coverage Night shift $(\mathrm{RR}=1.89 ; 95 \% \mathrm{CI}: 1.59-2.24)$. Age, gender, type of residency, and years of practice did not correlate with increased CT ordering. Conclusion: Higher rates of ordering were observed among physicians who reported increased concern with 1) risk of missing a diagnosis, 2) medico-legal risk, 3) patient wishes, and 4) what colleagues would do. Higher acuity shifts were significantly associated with increased CT ordering. We hypothesize that providing feedback to physicians could reduce utilization of $\mathrm{CT}$.

Keywords: imaging, utilization, practice variation

\section{LO19}

In patients presenting to the ED with bizarre behaviour, CT head scans do not yield acute findings and increase ED length of stay P. Ng, MD, M. McGowan, MHK, B. Steinhart, MD, University of Toronto, Toronto, ON

Introduction: A collaborative approach between the Emergency Department (ED) and Psychiatric Emergency Services is to medically "clear" a stable patient of organic pathology, including a computed tomography (CT) head scan, prior to psychiatric consultation. We sought to determine the impact of CT head on clinical management and ED length of stay (LOS) with patients presenting with bizarre behaviour. Methods: A single-site 5-year retrospective chart review of patients $\geq 18$ years, triaged as "mental health - bizarre behavior" (defined as deviating from normal cognitive behaviour) with a CT head scan in the ED was conducted. Exclusion criteria were focal neurologic deficits on exam, alternative medical etiology (i.e. delirium, trauma) and/or pre-existing CNS disease. Clinical, demographic, administrative, and neuroimaging data was extracted with $10 \%$ of charts independently reviewed by a staff Emergency Physician (EP) for inter-rater reliability. Results: 3316 cases were identified, $213(6 \%)$ had a CT head scan and 
$83(39 \%)$ met study inclusion criteria. No patients had acute findings on CT; 36 (43\%) had incidental findings that did not impact clinical management. EPs assessed promptly (mean, SD: 1:22 $\pm 1: 10 \mathrm{hr}$ ), requested 75 $(90 \%)$ consults $(3: 19 \pm 3: 51 \mathrm{hr})$, including $71(95 \%)$ to psychiatry, in which $47(57 \%)$ attended prior to CT result. Differences were noted in consultant assessment time [post CT 9:09 $\pm 10: 37 \mathrm{hr}$ vs prior 3:19 $\pm 7: 42$ ] and ED LOS [post CT 23:02 $\pm 17: 28 \mathrm{hr}$ vs prior 18:14 $\pm 18: 25 \mathrm{hr}$ ]. Conclusion: In patients presenting to the ED with bizarre behavior, CT head scans yielded no change in management but increased ED LOS due to delays in consultation. A larger multicenter study is underway to support these conclusions.

Keywords: mental health, patient flow, imaging - computed tomography

LO20

Utility of a 4-hour educational module in demonstrating transesophageal echocardiography (TEE) to TEE-naïve acute care providers

J. Pace, MD, S.L. McLeod, MSc, R. Arntfield, MD, University of Western Ontario, London, ON

Introduction: Emergency physicians (EPs) frequently employ transthoracic echocardiography (TTE) to assist in diagnosis, therapy and prognosis of critically ill patients with acute circulatory failure or shock. Transesophageal echocardiography (TEE) offers several advantages over TTE including reliable, continuous acquisition and superior image quality. Despite these advantages, use of TEE by EPs remains rare as methods of dissemination and training have not been described. The objective of this study was to evaluate TEE skill acquisition and retention by TEE-naïve EPs following a focused 4-hour workshop. Methods: Academic EPs were invited to participate in a 4-hour integrated didactic and simulation based workshop on the use of focused TEE for use in ED resuscitation. The didiactic and simulation-enhanced seminar emphasized TEE principles and basic 2D echocardiographic views obtained from 4 different vantage points; the mid-esophageal 4 chamber view, mid-esophageal long-axis view, transgastric short-axis view, and bicaval view. Following the training, participants engaged in an assessment of their abilities to carry out and interpret a focused TEE on a high fidelity simulator. Two expert echocardiographers independently reviewed the TEE images and graded the images as either clinically acceptable or unacceptable. A 6-week follow-up session assessed TEE skill retention. Results: Fourteen EPs credentialed in basic emergency point of care ultrasound participated in this study. Immediately following the seminar, $14(100 \% ; \mathrm{k}=1.0)$ and $10(71.4 \%, \mathrm{k}=0.65 ; 95 \% \mathrm{CI}$ : $0.21,1.0)$ were able to obtain an acceptable mid-esophageal 4 chamber and mid-esophageal long-axis view, respectively. $11(78.6 \%, \mathrm{k}=1.0)$ and $11(78.6 \%, \mathrm{k}=1.0)$ were able to obtain an acceptable transgastric short-axis and bicaval view, respectively. Median (IQR) time to study completion was $2.2(1.6,3.4)$ minutes. Ten participants engaged in skill retention assessment at 6 weeks and demonstrated sustained or improved performance, as determined by time to exam completion and number of acceptable views. Conclusion: EPs can successfully and expediently perform focused TEE using a simulator after a 4 hour curriculum and demonstrate retention of these skills at 6 weeks. Further, this study describes the first TEE curriculum for EPs and may serve as a model for widespread dissemination and training.

Keywords: echocardiography, transesophageal, education

LO21

Pilot prospective cohort study for the use of ultrasound guided 3 in 1 femoral nerve blocks in a tertiary care emergency department in patients presenting with a hip fracture

M.Y. Woo, MD, J. Bradley, MD, S. Moore, MD, A. Liew, MD, K. Lalonde, MD, MEd, I.G. Stiell, MD, MSc, University of Ottawa, Department of Emergency Medicine, Ottawa, ON
Introduction: Hip fractures are a painful condition that occurs in the elderly. The use of opioids in this population has disadvantages including respiratory depression and delirium. The goal of this study was to determine whether opioid administration is reduced in those patients who received an ultrasound guided 3in1 femoral nerve block (FNB). Methods: A historical cohort (Jan 2008-Mar 2009) using a formal health records review was compared to a prospective FNB cohort (Feb 2010-Jul 2011). Patients were included if they had an isolated hip fracture and excluded if less than 16 years, coagulopathic, or unable to rate their pain. The historical cohort was a random convenience sample of 100 patients prior to FNB enrolment. The prospective cohort was a convenience sample who underwent FNB by trained emergency physicians. The primary outcome was total dosage morphine equivalents at 1,4 , and 24 hours. Secondary outcomes included in-hospital complications and complications related to the block (local anesthetic systemic toxicity, nerve or vascular injury, infection). Data analysis included comparison of historical and FNB cohorts for baseline characteristics and outcome measures using standard chi-square with $95 \%$ confidence intervals. Results: The mean ages of the historical $(\mathrm{n}=100)$ and FNB $(\mathrm{n}=22)$ cohorts were 79.6 (range 41-99) and 84.0 years (range 70-97). In each cohort, $24.0 \%$ and $22.7 \%$ were male, respectively. There were no differences in vital signs and current use of opioids. The FNB cohort was more likely to have delirium risk factors $(68.2 \%$ vs $38.0 \%$, $\mathrm{p}=0.01)$ and respiratory risk factors $(54.6 \%$ vs. $27.0 \%, \mathrm{p}=0.01)$. Overall in-hospital complications were similar for both cohorts but urinary tract infections (UTI) and delirium were more common in the historical vs. FNB cohorts (UTI $46.2 \%$ vs. $9.1 \%$, p=0.002; delirium $55.8 \%$ vs. $18.2 \%, \mathrm{p}=0.003$ ). Mean morphine equivalents were similar at 1 hour [3.2mg $(95 \%$ CI $2.4-4.1)$ vs. $4.2 \mathrm{mg}(95 \% \mathrm{CI} 0.9-7.5)], 4$ hours [5.9mg (95\%CI $4.5-7.2)$ vs. $6.8 \mathrm{mg}(95 \% \mathrm{CI} 2.4-11.3)]$, and 24 hours [18.1mg (95\%CI $15.1-21.1)$ vs. $12.9 \mathrm{mg}(95 \%$ CI $2.3-23.5)]$. There were no complications from the FNB. Length of stay (LOS) was 28.2 (historical) vs. 18.3 (FNB) days. Conclusion: There were no complications from an ultrasound guided 3in1 FNB. In addition there were fewer cases of delirium, UTI, and shorter LOS in the FNB cohort despite similar morphine dosages. Larger trials are required to demonstrate the effectiveness of USG 3in1 FNBs in hip fractures.

Keywords: fracture, ultrasound, safety

\section{$\mathrm{LO} 22$}

Are interactive workshops effective at introducing medical students to PoCUS?

N. Packer, BSc, R. McLarty, BSc, C. Byrne, BSc, D. Thompson, MD, H. Hames, MD, R. Arntfield, MD, University of Western Ontario, London, $\mathrm{ON}$

Introduction: In keeping with expanding clinical application of pointof-care ultrasound (PoCUS), some medical schools have successfully incorporated ultrasound training into undergraduate curricula. Their experience suggests medical students efficiently acquire and retain ultrasound skills. Despite this, few opportunities for ultrasound training exist in the Canadian context. The objective of this study was to evaluate if medical students can generate and interpret clinically important ultrasound scans after participation in a short workshop series. Methods: Undergraduate medical students from five Ontario medical schools participated in a series of six ( 90 to 120 minute) bedside ultrasound workshops held sequentially as part of a two-day objectivebased curriculum, integrating hands-on learning with didactic teaching. Workshop topics included: abdominal aorta, hepatobiliary and renal; focused assessment with sonography for trauma; cardiac; lung and pleura; procedural guidance; and obstetric and gynecologic ultrasound. All scans were archived and independently scored by two expert physicians. Participants were evaluated on their ability to (1) interpret a series of scans by correctly identifying anatomy and common patho- 
physiological findings, and (2) perform five time-limited scans and correctly label anatomic landmarks in response to verbal prompt. Scan quality was evaluated according to: probe selection; marker orientation; image centering; and the correct generation and identification of anatomic landmarks. Results: Before the workshops, three of 35 students $(9 \%)$ had received formal PoCUS instruction with only two (4\%) performing greater than 10 scans. Following workshop completion, students were able to correctly identify anatomic landmarks $(83 \%)$ and common pathology $(88 \%)$ from selected clips. Students were able to generate and label high quality scans of the internal jugular vein and carotid artery (93\%), abdominal aorta (87\%), subxiphoid heart (74\%), right upper quadrant abdomen (73\%), and lung and pleura (70\%). Conclusion: Interactive workshops are an effective modality for teaching undergraduate medical students PoCUS. Students can rapidly acquire the skills needed to generate and identify clinically relevant anatomy using ultrasound. Our experience provides support for the pursuit of formal incorporation of PoCUS training within undergraduate medical curricula.

Keywords: point-of-care-ultrasound, undergraduate medical education

LO23

Does improvement in ED length of stay lead to improvements in quality of care?

M.J. Vermeulen, BScN, MHSc, A. Guttmann, MD, MSc, T. Stukel, PhD, M. Sivilotti, MSc, MD, B.H. Rowe, MD, MSc, J. Dreyer, MD, R. Bell, MDCM, MSc, M. Schull, MD, MSc, Institute for Clinical Evaluative Sciences, ON

Introduction: In 2009, Ontario launched a major policy initiative to reduce Emergency Department (ED) crowding, which included explicit targets for improvement in ED length of stay (LOS). We studied whether improvement in ED crowding was associated with better timeliness and quality of emergency care. Methods: Retrospective, structured medical record review in 24 Ontario hospitals from April 1, 2008 to March 31, 2009 and April 1, 2010 to March 31, 2011 (before and after the introduction of the ED Process Improvement Program). Eleven hospitals were selected as "improved" based on a $\geq 15 \%$ relative and $\geq 0.5$ hour absolute reduction in ED LOS from 2008 and 2010 and compared with 13 "not improved" sites randomly selected among hospitals with increased or no change in ED LOS. Quality indicators focused on acute myocardial infarction (AMI), asthma, and arm (children) and wrist (adult) fractures. Logistic regression models, accounting for correlation of outcomes within ED, controlled for daily shift-level ED crowding, patient age, sex, acuity, income, region of residence, and time of presentation. Results: There were 2,656 AMI, 2,738 asthma, 2,030 adult fracture, and 1,427 pediatric fracture charts. Baseline performance on quality indicators varied across and within medical conditions. Comparing the change in performance over time at sites where ED LOS improved with those where it did not, there were no differences in reperfusion (OR 0.58 [95\% CI 0.27 , 1.25]) or ASA (0.73 [0.51, 1.04]) for AMI; steroid within 60 minutes (OR $1.05[0.67,1.65])$, no chest x-ray (OR $1.25[0.87,1.80])$, corticosteroids at discharge (OR $0.81[0.59,1.11])$, or documented discharge instructions (OR $0.87[0.54,1.40]$ ) for asthma; or pain assessment (OR $0.96[0.67,1.36]$; OR $1.15[0.77,1.73]$ ), analgesic/splint (OR 0.76 [CI $0.37,1.54]$; OR $1.34[0.58,3.11]$ ), or discharge instructions (OR 1.02 $[0.64,1.65]$; OR $1.45[0.54,3.92])$ for arm/wrist fracture among adults and children, respectively. In sites with improved ED LOS, asthma patients were more likely to receive a lung function test (OR 2.54 [1.27, 5.07]). Conclusion: Reductions in ED LOS were not associated with improved performance on several standard indicators of quality of care across a range of conditions. Thus, while policies to improve ED LOS are needed, they do not appear to improve the management of pain, asthma or AMI in the ED.
Keywords: quality indicators, emergency department overcrowding, health policy

\section{LO24}

Efficacy of phone follow-up in reducing adverse events in the emergency department: a pilot project

L.A. Calder, MD, MSc, N. Larocque, BHSc, S. Calder-Sprackman, MD, R. Cagaanan, BSc, J. Zlepnig, BA(Hons), BScN, A. Cwinn, MD, I.G. Stiell, MD, MSc, A. Forster, MD, Ottawa Hospital Research Institute, Clinical Epidemiology Program, Ottawa, ON

Introduction: Return emergency department (ED) visits are frequent ( $20 \%$ of high acuity patients) and may be due to adverse events (AEs), adverse outcomes related to healthcare received. The objective of this study was to determine whether an interactive voice response system (IVRS) for telephone follow-up of discharged high acuity ED patients reduced the occurrence of AEs. Methods: We conducted this before and after interventional study at the Ottawa Hospital Civic Emergency Department. In phase one (before IVRS), we assessed for AEs among 500 consecutively discharged high acuity ED patients. We defined high acuity as patients who were triaged to a monitored area or a stretcher area of the ED. In phase two (after IVRS), we implemented a computerized telephone follow-up system for 500 consecutive high acuity discharged ED patients. These patients were asked by the IVRS: 1) are you having a health problem? 2) would you like to speak to a nurse? Patients who responded yes received a structured telephone interview by a trained nurse. We also searched health records for flagged outcomes which included: deaths, admissions to hospital, return ED visits, or clinic visits within 14 days. Three trained emergency physicians independently reviewed case summaries and determined AE occurrence using a structured, piloted process. We analyzed outcomes using descriptive statistics. Results: Of the 999 patients enrolled, patient characteristics of age, sex and CTAS score were comparable in both phases. Of 504 eligible phase 2 patients for IVRS, 9 were excluded due to restricted or unavailable telephone numbers. Of the 495 remaining patients, 393 (79.4\%) were successfully contacted by IVRS. Of these, $89(22.6 \%)$ wanted to speak to a nurse. In phase 1 , we detected 162 flagged outcomes and in phase 2, we detected 260 $(\mathrm{p}<0.0001), 105$ of which were found on telephone follow-up. We found 31 AEs (19.0\%) in phase 1 and $21(8.1 \%)$ in phase 2. Overall, there was 1 death, 25 admissions, 23 return ED visits and 2 clinic visits. Conclusion: IVRS can be a useful tool for post-discharge followup of select ED populations. We were able to identify more flagged outcomes and noted a reduced numbers of AEs with this method of following up high risk ED patients.

Keywords: emergency department, adverse events, phone follow-up

\section{LO25}

Canadian EM trainees' coping with fatigue and shiftwork: results of a national survey

J.R. Frank, MD, MA(Ed), K. Imrie, MD, L.J. Gorman, MA, A. Ronson, MSc, S. Taber, MHA, Royal College of Physicians and Surgeons

Introduction: Resident duty hours $(\mathrm{RDH})$ are being debated worldwide with respect to issues such as patient safety, educational effectiveness, and resident wellness. In 2013, the Canadian national task force on RDH surveyed residents and other stakeholders with respect issues of safety and wellness. However, it is not known if Emergency Medicine (EM) trainees differ in their perspectives versus their peers in other disciplines. In this subgroup analysis, we describe the patterns reported by EM residents versus the general population of resident respondents nationally. Methods: We created an RDH questionnaire with the input of the major medical education organizations on the task 
force. Residents were asked to provide their perspective on work stress, medication use to combat fatigue, and fatigue-related adverse events. The survey was distributed online to all active trainees in Canada ( $\mathrm{n}=12672)$ via provincial housestaff organizations. Results: 3610 Canadian residents completed the entire survey (28.5\%), with 114 identifying themselves as EM trainees (3.2\%). EM and other trainees reported similar proportions of dissatisfaction with stress levels $(43.9 \%$ vs $48.9 \%, \mathrm{p}=0.51)$. EM trainees reported more prolonged sleep deprivation $(78.1 \%$ vs $70.2 \%, \mathrm{p}=0.07)$. More EM residents endorsed using caffeine to stay awake $(86.8 \%$ vs $74.2 \%, \mathrm{p}=0.002)$, using other medications to stay awake $(9.6 \%$ vs $5.2 \%, \mathrm{p}=0.038)$, and using medications to sleep $(28.9 \%$ vs $20.2 \%, \mathrm{p}=0.024)$. With respect to fatigue-related adverse events, more EM trainees reported being involved in a near-miss collision driving home $(34.2 \%$ vs $23.6 \%$, $\mathrm{p}=0.009$ ) and a medical error with potential for serious patient harm (18.6\% vs $10.5 \%, \mathrm{p}=0.006)$. Only $1 \mathrm{EM}$ trainee reported having an actual motor vehicle collision due to duty hour fatigue. Conclusion: EM trainees report a significantly greater impact of fatigue on their lives and their patients. Leaders in EM education should urgently review EM RDH policies in light of these findings.

Keywords: resident duty hours, EM education, safety

LO26

Targeted temperature management: exploring the association between processes of care and outcomes after out-of-hospital cardiac arrest

S. Lin, MDCM, MSc, D.C. Scales, MD, PhD, P. Dorian, MDCM, MSc, A. Kiss, PhD, M.R. Common, BSc, S.C. Brooks, MD MHSc, S.G. Goodman, MD, MSc, L.J. Morrison, MD, MSc, Division of Emergency Medicine, Department of Medicine, University of Toronto, Toronto, ON

Introduction: Randomized trials have demonstrated that Targeted Temperature Management (TTM) improves functional survival in patients after out-of-hospital cardiac arrest (OHCA). However, the optimal process of implementation and delivery remains unclear, specifically how fast, how cold or how long to cool. The objective of this study was to evaluate the association of these processes of care with survival and neurological function in patients after OHCA. Methods: We conducted a retrospective cohort study on consecutive adult ( $\geq 18$ years) non-traumatic OHCA patients from November 1, 2007 to January 31,2012 . Eligible patients who were treated with TTM after return of spontaneous circulation were included. The primary and secondary outcomes were in-hospital survival and good neurological status (Modified Rankin Scale of 0-3) at hospital discharge, respectively. Bivariate and generalized estimating equation analyses were used to evaluate the associations between processes of care variables and patient outcomes. Results: There were 5770 consecutive OHCA patients, of whom $747(12.9 \%)$ patients were eligible and received TTM; 365 (48.9\%) patients survived to hospital discharge, of whom $241(66.0 \%)$ patients had good neurological outcomes. After adjusting for the Utstein variables, a higher temperature prior to cooling was associated with improved survival [OR $1.26 \mathrm{per}^{\circ} \mathrm{C}, 95 \% \mathrm{CI} 1.09-1.46$, $\mathrm{p}=0.002$ ] and neurological outcomes [OR 1.27 per ${ }^{\circ} \mathrm{C}, 95 \%$ CI 1.08 $1.50, \mathrm{p}=0.004]$. Moreover, a slower rate of cooling was associated with improved survival [OR 0.73 per ${ }^{\circ} \mathrm{C} / \mathrm{hr}, 95 \%$ CI $0.54-1.00, \mathrm{p}=0.049$ ] and neurological outcomes [OR 0.74 per ${ }^{\circ} \mathrm{C} / \mathrm{hr}, 95 \%$ CI $0.57-0.97$, $\mathrm{p}=0.03]$. The location of cooling initiation, coldest temperature, and cold duration $\leq 34^{\circ} \mathrm{C}$ were not associated with outcomes. Conclusion: A higher baseline temperature prior to cooling and a slower rate of cooling were associated with both improved survival and neurological outcomes. This may reflect the complex relationship between the extent of brain injury causing hypothalamic dysfunction and impaired thermoregulation, and the cooling techniques used in patients after cardiac arrest.

Keywords: cardiac arrest, post-cardiac arrest care, targeted temperature management

\section{LO27}

Innovative use of AED by RNs and RTs during in-hospital cardiac arrest

H. Buhariwalla, MSc, C. Vaillancourt, MD, MSc, CSPQ, R. Marcantonio, P. Pageau, MD, University of Ottawa, Department of Emergency Medicine, Ottawa, ON

Introduction: Most in-hospital defibrillators are equipped with an Automated External Defibrillator (AED) mode, yet nurses and respiratory therapists (RT) trained in their use are not legally permitted to use them during in-hospital cardiac arrests in the absence of a medical directive. We sought to measure baseline in-hospital cardiac arrest response characteristics and evaluate time delays amenable to AED use by nurses and RTs. Methods: We performed a health record review of all in-hospital cardiac arrests occurring at The Ottawa Hospital in 2012. We used ICD-10 codes to identify potentially eligible cases, and included in-hospital cardiac arrests for which resuscitation was attempted, including those re-arresting following prehospital return of spontaneous circulation (ROSC). We developed a standardized and piloted data collection tool based on the Utstein reporting guidelines for in-hospital cardiac arrest. We obtained consensus on all data definitions before initiation of data extraction by a trained investigator. We report descriptive statistics. Results: There were 120 in-hospital cardiac arrests for which resuscitation was attempted with the following characteristics: mean age $69.7,59.2 \%$ male, $71.7 \%$ witnessed, initial rhythm PEA $42.5 \%$, VF/VT $25.8 \%$, asystole $19.2 \%$, ROSC $64.2 \%$, and survival to hospital discharge $21.7 \%$. Most survivors $(61.5 \%)$ were discharged home. Most cardiac arrests $(30.0 \%)$ were respiratory in nature. Resuscitation was initiated in $10.0 \%$ of patients with a documented DNR, and we observed poor adherence to 2010 resuscitation guidelines $(40.0 \%$ received bicarbonates). Cardiac arrests commonly occurred in medical/surgical wards $31.7 \%$, the emergency department (ED) $27.5 \%$, critical care units $19.1 \%$, and the operating room (OR) $10.8 \%$. The mean time interval between cardiac arrest discovery and first defibrillation was 8 minutes; it was 6 minutes in the ED and the OR, 8 minutes in critical care units, and 11 minutes on medical wards. Conclusion: Very few institutions monitor the clinical care and outcomes for in-hospital cardiac arrest victims. We observed poor adherence to the 2010 resuscitation guidelines, challenges in identifying DNR patients at the time of cardiac arrest, and a very long delay before defibrillation occurred in non-monitored areas, where most cardiac arrests occurred. The use of AEDs by RNs and RTs, could potentially lead to significant reductions in time to first shock and improved survival outcomes.

Keywords: cardiac arrest, automated external defibrillator

\section{LO28}

Was the return of spontaneous circulation protocol effective? Survival to discharge and adherence to the protocol through a health record review

J. Moore, MD, P. Pageau, MD, C. Poulin, BScN, A. Willmore, MD, S. Duncan, M. Le May, MD, C. Osborne, BSc, C. Vaillancourt, MD, MSc, The Ottawa Hospital, Ottawa, ON

Introduction: Out-of-hospital cardiac arrest (OHCA) affects over 40,000 victims annually in Canada. The Ottawa Hospital (TOH) and the University of Ottawa Heart Institute (UOHI) recently designed and introduced the Code ROSC (Return of Spontaneous Circulation) proto- 
col, which consists of initiation of therapeutic hypothermia in the emergency department (ED), dual Cardiology and Critical Care urgent consultation and early disposition and access to time sensitive interventions (e.g. PCI, intra-aortic balloon pump, trans-venous pacemaker). In a population of OHCA patients presenting to the ED, we sought to determine adherence to the Code ROSC protocol, and its impact on survival to hospital discharge. Methods: We conducted a before-after health record review for OHCA patients receiving care at TOH ED and the UOHI 12 months before and 12 months after the introduction of the Code ROSC protocol in September 2011. We reviewed ambulance call reports provided by the Regional Paramedic Program for Eastern Ontario (RPPEO) in addition to patient records from $\mathrm{TOH}$ and the UOHI using a piloted data collection tool. Adult OHCA patients with witnessed VF/VT and ROSC are eligible for the Code ROSC protocol, while those with full neurological recovery on ED arrival, with $>30$ min from arrest to ACLS, with $>60$ min from arrest to ROSC, with STEMI, and those with refractory shock, hypoxia, or severe coagulopathy are not. Results: Characteristics of the 247 included patients were: mean age 65 , male gender $64.0 \%$, witnessed $83.0 \%$, bystander CPR $33.2 \%$, VF/VT $40.5 \%$, residential location $55.0 \%$, and time from 911 call to scene 7:42 min. Intention to treat analysis revealed an absolute increase in survival to hospital discharge of $5.9 \%$ (95\% CI $-6.7-18.6 \%$ ) during the intervention period. Among those where the Code ROSC was implemented, absolute increase in survival was $29 \%(95 \%$ CI $-10-69.4 \%)$ for the protocol eligible patients, and $7.4 \%$ (95\% CI $-24.9-39.7 \%)$ for the protocol ineligible patients. The protocol was activated appropriately in $90 \%$ of the eligible patients and it was appropriately not activated in $86 \%$ of the ineligible patients. Conclusion: The Code ROSC protocol was successfully implemented with satisfactory adherence to its inclusion and exclusion criteria. In this pilot evaluation phase, the Code ROSC protocol resulted in a clinically significant increase in survival to hospital discharge. The Code ROSC protocol is contributing to improved post cardiac arrest care at TOH and the UOHI.

Keywords: cardiac arrest, therapeutic hypothermia, return of spontaneous circulation

\section{LO29}

Multicentre implementation of the Canadian C-Spine Rule by emergency department triage nurses

I.G. Stiell, MD, MSc, C.M. Clement, M. Lowe, C. Dain, J. Miller, B. Bailey, K. Posselwhite, S. Armstrong, J. Langlais, K. Ruddy, BScN, S. Thorne, A. Armstrong, Ottawa Hospital Research Institute, Ottawa, ON

Introduction: The Canadian C-Spine Rule (CCR) has been widely applied by ED physicians to safely reduce use of c-spine imaging. Our objective was to evaluate the impact of CCR implementation by ED triage nurses. Methods: We conducted a multi-phase multicentre cohort study at 9 teaching hospitals and enrolled consecutive, alert and stable adult trauma patients presenting to the ED with neck pain or with c-spine immobilization. During Phase 1, ED nurses were trained and then had to demonstrate competence by accurately assessing 10 patients prior to being certified. During Phase 2, certified nurses were empowered by a medical directive to "clear" the c-spine of patients, allowing them to remove c-spine immobilization and to triage to a less acute area. We conducted simple descriptive statistics. Results: In Phase I, 3,098 patients were evaluated by 312 nurses. In Phase II, 1,408 patients were enrolled by 180 nurses ( 898 ambulance and 510 ambulatory patients, respectively) with mean age 43.1 years, female $52.3 \%$, MVC $80.7 \%$, c-spine injury $1.1 \%$. For the 806 immobilized ambulance patients, $41.1 \%$ had immobilization removed (compared to $0 \%$ at baseline), $24.1 \%$ were able to sit up, and $16.1 \%$ were moved to an ambulatory area. No c-spine injuries were missed by the nurses. In only $1.3 \%$ of cases did the nurse indicate that she/he was uncomfortable following the CCR. Conclusion: We clearly demonstrated that ED triage nurses can effectively implement the CCR, leading to more rapid and comfortable management of patients without any missed fractures or threat to patient safety.

Keywords: cervical spine, nursing, decision rules

\section{LO30}

Delayed hemothorax following minor thoracic injuries

M. Emond, MD, MSc, J. Chauny, MD, MSc, L. Vanier, MD PhD, N. Le Sage, MD, MSc, Université Laval, Québec, QC

Introduction: Patients admitted to emergency department (ED) for minor thoracic injuries (MTI) are possibly at risk of delayed hemotho$\operatorname{rax}(\mathrm{DHx})$. Little is known about the epidemiology and characteristics of patient with MTI treated on an out-patient basis that have DHx. Objectives: We aimed to evaluate the incidence of DHx post-ED and the risk factors associated. Methods: Methods: Design: A prospective cohort study was conducted in four Canadian ED, from March 2008 to August 2010. All consecutive patients, 16 years and older, with MTI and discharged from ED were screen and eligible. All initial chest XRay were reported normal. A standardized clinical and radiological evaluation was performed upon initial ED visit and repeated at 1 and 2 weeks. Phone interviews were done at 30 and 90 days. Outcome: the presence of delayed radiological diagnosis of hemothorax. Data Analyses: Univariate and multivariate analyses were realized to obtain outcome measures. Results: Of the 989 recruited participants, 254 $(25.7 \%)$ were 65 y.o. or more, $610(6.1 .6 \%)$ were male and 304 $(30.9 \%)$, at least one rib fracture confirmed on radiological report. A simple level-ground fall was the most frequent mechanism of injury( $\mathrm{n}=325(32.8 \%))$. Overall, $118(11.9 \%)$ had developed a DHx within the first 14 days of follow-up. Our multivariate model, after controlling for patient's age and co-morbidities, found pre- radiologically confirmed rib fracture, motor vehicle accident and use of ASA, to significantly increase risk of DHx. OR were 4.4 (95\% CI 2.6 - 7.1), 1.9 (1.1- 3.2) and 1.6 (95\% CI 0.9 -2.9), respectively. Conclusion: This prospective cohort study on non-hospitalized patients with MTI revealed a significant incidence of delayed hemothorax. Our results support the development of clinical decision rules to orient tailored follow-up for MTI patients in ED.

Keywords: delayed hemothorax, minor thoracic injury

\section{LO31}

Is hockey the most dangerous pediatric sport? An evaluation of pediatric sport-related injuries treated in Nova Scotia

R. Green, MD, M. Erdogan, PhD, M. Butler, BSc, Dalhousie University, Halifax, NS

Introduction: Hockey-related injuries are considered a leading cause of hospitalization for children and youth in sports. The objective of this study was to identify the incidence, characteristics, and mechanisms of major sport-related injuries among pediatric patients treated in Nova Scotia. Methods: This study was a retrospective case series. We hypothesized that hockey is the most common cause of sport-related injuries for pediatric patients between the ages of 0 and 19 years in Nova Scotia. To test our hypothesis we extracted, combined, and analyzed data on sport-related injuries seen in Nova Scotia between 2000 and 2012 from the Nova Scotia Trauma Program Registry (NSTPR). Results: In total, 111 children aged 3 to 18 years sustained a major traumatic injury in Nova Scotia, with $83.8 \%$ being male. The highest proportion of sport-related injuries resulted from cycling $(63,56.8 \%$; 2.28 per 100,000 pediatric population), followed by hockey $(9,8.1 \%$; 0.33 per 100,000 pediatric population) and skiing $(7,6.3 \% ; 0.25$ per 
100,000 pediatric population). Traumatic brain injuries (TBI) accounted for $52.3 \%$ of all injuries. The sport with the highest incidence of TBI was baseball $(4,100 \%)$, followed by golf $(4,100 \%)$ and cycling $(54.0 \%)$. The incidence of TBI in the hockey group was $33.3 \%$. There were five deaths, two in the cycling group $(0.072$ per 100,000 population), two in the skateboarding group $(0.072$ per 100,000 pediatric population), and one in the swimming group ( 0.036 per 100,000 pediatric population). The rate of ICU admission in the hockey group was $44.4 \%$, compared to $36.3 \%$ in all other groups. Conclusion: This is the first analysis evaluating data from the NSTPR. The results reveal that cycling is the largest contributor to pediatric traumatic injury, followed by hockey. Hockey is a principal contributor of traumatic brain injury, and is associated with a significant number of traumatic brain injuries, and a greater rate of ICU admission. These findings can inform strategies for provincial injury prevention programs.

Keywords: contact sport

\section{LO32}

Ultrasonography of the optic nerve sheath diameter for detection of raised intracranial pressure: a systematic review and metaanalysis

R. Ohle, MA, MB, BCh, BAO, S. McIsaac, MEd, MB, BCh, BAO, M.Y. Woo, MD, J.J. Perry, MD, MSc, University of Ottawa, Department of Emergency Medicine, Ottawa, ON

Introduction: The rapid diagnosis of raised intracranial pressure (ICP) is an important problem in the emergency department (ED) and intensive care unit (ICU). The optic nerve sheath is continuous with the subarachnoid space, thus an increase ICP results in a corresponding increase in optic nerve sheath diameter. Ultrasonography (US) has been proposed as a fast, point of care measure of the optic nerve sheath diameter. The objective of this systematic review and meta-analysis was to assess the test characteristics of US of the optic nerve sheath diameter as compared to computed tomography (CT) in predicting raised ICP in the ED and ICU with suspected raised ICP. Methods: We searched Pubmed, Medline, Embase and the Cochrane database from 1968 to August 2013 and performed hand searches. No restrictions for language or study type were imposed. Relevant studies were reviewed and data was extracted by two independent reviewers. A positive test was defined as optic nerve sheath diameter $>5 \mathrm{~mm}$ in adults, $>4.5 \mathrm{~mm}$ in children $1-17$ years and $>4 \mathrm{~mm}$ in children $<1$ year. Study quality was assessed using the QUADAS tool. We calculated kappa agreement for study selection and evaluated clinical and quality homogeneity before meta-analysis. Results: We reviewed 1,214 studies and selected 45 for full review. Twelve studies with 478 participants were included (kappa, 0.9). Ocular US yielded a sensitivity of $95.6 \%(95 \%$ CI, 87.7-98.5), specificity $92.3 \%$ (95\% CI, 77.9-98.4) for raised ICP. LR+ 12.5 (95\% CI, 4.16-37.5) and a LR- 0.05 (95\% CI, 0.02-0.14). Average quality using the QUADAS tool was $7.4 / 11$. There was a moderate to high level of heterogeneity based on prediction ellipse area and the variance logit of sensitivity (2.2), and specificity (2.8). Conclusion: Ocular US shows good diagnostic test accuracy for detection of raised ICP when compared to CT. This non-invasive point of care method could lead to more timely interventions for raised ICP, be used in centers without CT, during patient transport or as part of a diagnostic protocol to reduce the number of CTs performed.

Keywords: ocular ultrasonography, intracranial pressure, optic nerve sheath diameter

LO33

Use of emergency physician performed ultrasound for the detection of lower extremity deep venous thrombosis

T.V. Lam, MD, J. Theoret, MD, A. Lund, MD

Vancouver General Hospital, Vancouver, BC
Introduction: The body of evidence supporting the use of emergency physician performed ultrasound (EPPU) in the assessment of suspected lower extremity deep venous thrombosis (DVT) is continually growing. Multiple studies conducted in the USA and internationally have demonstrated high sensitivities and specificities $(92.7-96.5 \%$ and $95.3-$ $97.0 \%$ respectively). However, there are concerns that the results of these studies are not generalizable because of the low number of ED physicians performing the US and their higher level of training in EPPU. Since the experience of Canadian ED physicians with advanced EPPU is so heterogeneous, it would be difficult for the results of the existing studies to be widely applied in most EDs in Canada. The objective of the study was to determine the sensitivity and specificity of US performed by a heterogeneous group of ED physicians after a 30-minute training session compared to formal ultrasound (US) for the assessment of DVT. Methods: ED physicians attended a 30-minute training session. Any patient $>18$ yo presenting with suspected DVT was consented. Prior to the EPPU, physicians were asked to document whether they planned to anticoagulate the patient based on their risk factor profile and clinical presentation. The EPPU included a 2-point compression test at the common femoral vein and popliteal vein. The findings were subsequently compared to the formal US. Results: Thirty physicians were involved in the study. Between March 2012 and September 2013, 176 patients were enrolled. Ultimately, 154 were included in the final analysis because 15 were lost to follow-up, 4 did not have consent forms on record, and 3 were assisted by unauthorized staff. Of the 154 patients, 23 had a formal diagnosis of DVT. There were discrepancies between the EPPU and the formal US in 13 of the studies: 5 false negatives and 8 false positives. The sensitivity of EPPU for DVT was $78.3 \%(95 \% \mathrm{CI}: 56.3-92.5 \%)$ and the specificity was 93.9\% (95\%CI: 88.3-97.3\%). Conclusion: Two-point compression US performed by a heterogeneous group of ED physicians receiving a 30minute training session had insufficient sensitivity and specificity to be a sole diagnostic modality for the evaluation of DVT. More extensive training, ongoing feedback and quality assurance may be required to improve the ability of a general group of ED physicians to adequately diagnosis or rule out DVT in the ED.

Keywords: emergency physician performed ultrasound, emergency department ultrasound, point-of-care ultrasound

\section{LO34}

Predictors of door-to-imaging time in emergency headache patients with suspected subarachnoid hemorrhage

M. Khan, MSc, J.J. Perry, MD, MSc, M. Sivilotti, MSc, MD, University of Ottawa, Department of Emergency Medicine, Ottawa, ON

Introduction: Optimal diagnostic sensitivity of imaging depends on minimal door-to-imaging time (DIT) among patients with suspected subarachnoid hemorrhage (SAH). Our objective was to assess the impact of clinical characteristics and hospital system variables on DIT among patients presenting with headache at risk for SAH. Methods: This was a prospective cohort study at the emergency departments (EDs) of 11 university affiliated tertiary care teaching hospitals in Canada from November 2000 to July 2010. Subjects were neurologically intact adults with an acute, non-traumatic headache in whom CT was performed to rule out SAH. Data were collected on predictors of door-to-imaging time. Data included clinical characteristics, mode of transport to the ED and triage risk assessment (Canadian Triage Acuity Scale, CTAS). Our primary outcome measure was time from hospital registration to CT. Multivariable Cox proportional hazards modelling was performed to identify predictors of DIT. Results: Our study included 2,412 ED headache patients. Of these, 194 had a diagnosis of SAH. Subjects with SAH were older $(52.7$ yrs vs 44.2 yrs, $\mathrm{p}<0.0001)$ and arrived to the ED with ambulance more often $(56.2 \%$ vs $21.7 \%$, p $<0.0001)$ than subjects without SAH. DIT was shorter in patients with 
SAH $(1.9$ hours $(\mathrm{IQR}=1.7)$ vs. 2.5 hours $(\mathrm{IQR}=2.4))$. In the multivariable model, variables associated with a decreased DIT included transfer from another $\mathrm{ED}(\mathrm{HR}=2.00,95 \% \mathrm{CI}=1.72-2.30)$, increased systolic blood pressure $(\mathrm{HR}=1.04 / 10 \mathrm{mmHg}, 95 \% \mathrm{CI}=1.02-1.05)$, higher triage acuity (CTAS $1-2 \mathrm{HR}=1.52,95 \% \mathrm{CI}=1.32-1.76$; CTAS $3=1.25,95 \% \mathrm{CI}=1.10-1.41$; both relative to CTAS $4-5)$, arrival by ambulance $(\mathrm{HR}=1.21,95 \% \mathrm{CI}=1.09-1.35)$ and male gen$\operatorname{der}(\mathrm{HR}=1.14,95 \% \mathrm{CI}=1.05-1.23)$. Conclusion: Imaging occurs more quickly in patients with features suggestive of SAH, perhaps reflecting prioritizing investigation in patients with a more concerning presentation. The increased sensitivity of $\mathrm{CT}$ with shorter delays to imaging may be partially explained by a larger volume or severity of $\mathrm{SAH}$, even though in-hospital delays to imaging are much shorter than the delay to presentation. As a result, merely expediting imaging in all headache patients may not ensure the near-perfect sensitivity of sub- 6 hour CT is preserved.

Keywords: subarachnoid hemorrhage, computed tomography, headache

\section{LO35}

Ketamine-propofol vs propofol alone for procedural sedation in the emergency department: a systematic review and meta-analysis J. Yan, MD, MSc, S.L. McLeod, MSc, University of Western Ontario, London, ON

Introduction: Patients in the emergency department (ED) often require procedural sedation and analgesia (PSA) while undergoing painful procedures. Propofol is a commonly used pharmacologic agent used for PSA, but can potentially cause adverse events such as respiratory depression and hemodynamic compromise. The combination of ketamine-propofol (KP) is an alternative that provides a theoretical reduction in adverse events compared to propofol. The objective of this study was to determine if KP reduces the frequency of adverse respiratory events in patients undergoing PSA in the ED compared to propofol alone. Secondary objectives were to compare the proportion of overall adverse events and procedure time between groups. Methods: Electronic searches of Medline, EMBASE, Cochrane Central Register of Controlled Trials, and CINAHL were conducted and reference lists of relevant articles were hand searched. Randomized controlled trials (RCTs) published in the English language comparing the use of KP versus propofol alone for PSA in the ED were included. Two reviewers independently screened titles and abstracts, assessed study quality and extracted data. Where appropriate, data were pooled using randomeffects models and reported as risk ratios (RR) with $95 \%$ confidence intervals (CIs). Median procedure time was reported for three RCTs. This data could not be pooled and is presented descriptively. Results: Six RCTs were included with a combined total of 707 patients $(\mathrm{KP}=$ 369 , propofol $=338$ ). Five RCTs reported the proportion of adverse respiratory events; the pooled estimate showed that there was no difference when KP (28.4\%) was compared to propofol (35.1\%) alone (RR: $0.81 ; 95 \%$ CI: $0.66,1.00)$. There was no difference with respect to the proportion of overall adverse events (RR: $0.84 ; 95 \%$ CI: $0.69,1.03$ ). Procedure time was similar when the two groups were compared. Conclusion: Although the premise of combining ketamine with propofol is based upon the many theoretical synergies that exist between these two agents, KP does not reduce the proportion of adverse events for PSA in the ED when compared to propofol alone. Additionally, procedure time remains similar when the two groups are compared.

Keywords: ketamine-propofol, propofol, procedural sedation

LO36

Pediatric pain management: the emergency medicine residents' perspectives
H. Ali, MDCM, C. Meyers, MDCM, J. MacLellan, MD, J. Kircher, MD, S. Ali, MDCM, McGill University, Montréal, QC

Introduction: Pain is a component of up to $80 \%$ of all emergency department (ED) visits. Oligoanalgesia, the under-treatment of pain, is a recognized problem in the $\mathrm{ED}$ and can have numerous detrimental effects for the pediatric patient. Surveying emergency medicine residents, our primary objectives were to describe the extent and type of training received, management approach to common painful pediatric presentations, level of comfort in assessing and treating acute pain, perceived facilitators and barriers to optimally managing pain, and attitudes towards managing such pain in the ED. Methods: A descriptive, cross-sectional survey was administered to all Royal College Emergency Medicine, Pediatric Emergency Medicine, and Canadian College of Family Physician's EM trainees at three universities. A novel survey tool was created and distributed from May to June 2013. Surveys were completed online, using a modified Dillman approach. Results: Our response rate was $46 \%(56 / 122)$. Forty-five percent $(25 / 55)$ of residents had not received any training in pediatric pain assessment. All years of residents reported they were uncomfortable with the assessment of pain in $0-2$ year olds $(\mathrm{p}=0.07)$; for patients aged 2-12 years, level of comfort with pain assessment increased with years of training $(\mathrm{p}=0.02)$. When assessing pain in children with disabilities, $83 \%(45 / 54)$ of residents were 'extremely' or 'somewhat' uncomfortable and $65 \%(47 / 55)$ of residents had not received any training on how to assess their pain. Sixty nine percent $(38 / 55)$ of residents had received training on how to treat pediatric pain. All residents were more comfortable using pain medication for a 9 year old, as compared to a 1 year old (oral oxycodone $\mathrm{p}=0.00003$, oral morphine $\mathrm{p}=0.00002$, IV morphine $\mathrm{p}=0.004)$. Ninety-eight percent $(54 / 55)$ of residents felt that it was 'extremely' or 'somewhat' important to receive education about pediatric pain. The preferred methods to learn children's pain management were role modeling $(61 \%)$ and lectures $(57 \%)$. Challenges to optimal pediatric pain management were non-verbal, disabled, and young children. Conclusion: Pediatric pain management education is important to Canadian EM residents. Residents recall receiving sub-optimal training on this important topic. They are especially uneasy in the management of younger children and those with disabilities. This study's findings can be used to inform post-graduate curriculum planning, in order to optimize pediatric pain assessment and treatment education.

Keywords: postgraduate education, pain management, pediatrics

\section{LO37}

A randomized double-blind trial comparing the effect on pain of an oral sucrose solution versus placebo in children 1 to 3 months old needing venipuncture

M. Desjardins, MD, N. Gaucher, MD, MSc, S. Curtis, MD, MSc, S. LeMay, PhD, D. Lebel, BPharm, MSc, S. Gouin, MDCM, CHU SteJustine, Montréal, QC

Introduction: Oral sweet solutions have an analgesic effect in neonates. There have been a limited number of published clinical trials in older infants for the commonly performed painful procedures in any setting. These few studies have shown conflicting results. We aim to compare the efficacy of an oral sucrose solution versus placebo in reducing pain in children 1 to 3 months of age during venipuncture in the Emergency Department (ED). Methods: A single-center, randomized, double-blind, placebo controlled clinical trial was conducted in a tertiary care pediatric university-affiliated ED. All infants 1 to 3 months of age requiring venipuncture were randomly allocated to receive $2 \mathrm{ml}$ of $88 \%$ sucrose solution or $2 \mathrm{ml}$ of a placebo solution orally, two min before the procedure. The primary outcome measure was the difference in pain levels 
during venipuncture as assessed by the Face, Legs, Activity, Cry and Consolability Pain Scale (FLACC) 1 min post venipuncture. Secondary outcome measures were the difference in pain levels using the Neonatal Infant Pain Scale (NIPS), crying time and variations in heart rate. Results: Eighty-seven participants were recruited: 45 sucrose, 42 placebo. Overall, both groups had similar baseline demographic and clinical characteristics, including administration of pacifier and other analgesia. The mean difference in FLACC pain scores compared to baseline was $2.07 \pm .77$ in the placebo group vs. $1.36 \pm .59$ in the sucrose group $(\mathrm{p}=.49)$. For the NIPS pain score, it was $1.73 \pm .62$ in the placebo group vs. $0.75 \pm$ .58 in the sucrose group $(p=.36)$. The difference in the mean crying time following venipuncture was statistically significant between both groups $(69 \pm 13$ seconds in the placebo group vs. $49 \pm 13$ in the sucrose group, $\mathrm{p}=.04$ ). No significant difference was found in the difference of participants' heart rates 1 min post venipuncture compared to baseline $(18 \pm 4$ beats/min for placebo vs. $16 \pm 4$ for sucrose, $\mathrm{p}=.74)$. No significant adverse effects were reported. The overall blinding was successful. Conclusion: In children 1 to 3 months of age undergoing venipuncture in the ED, administration of an oral sweet solution did not statistically decrease pain scores as measured by the FLACC and NIPS scales or participants' heart rates. However, crying time was significantly decreased by providing sucrose instead of a placebo.

Keywords: pain, oral sweet solutions, infants

\section{LO38}

Emergency medicine residents' beliefs about contributing to an online collaborative slideshow

P.M. Archambault, MSc, MD, J. Thanh, D. Blouin, MD, MHPE, S. Gagnon, MA, J. Poitras, MD, R.B. Fountain, PhD, R. Fleet, MD, PhD, A. Bilodeau, MSc, T.H. van de Belt, PhD, F. Légaré, MD, PhD, Department of Family Medicine and Emergency Medicine, Université Laval, Québec, QC

Introduction: Collaborative writing applications (CWAs) such as Google Docs can improve skill acquisition, knowledge retention and collaboration in medical education. Using CWAs to support the training of residents offers many advantages, but stimulating them to contribute remains challenging. The purpose of this study was to identify emergency medicine (EM) residents' beliefs about their intention to contribute summaries of landmark articles to a Google Docs slideshow while studying for their Royal College exam. Methods: Using the Theory of Planned Behavior, the authors interviewed Canadian EM residents about contributing to the slide show. Residents were asked about behavioral beliefs (advantages/disadvantages), normative beliefs (positive/negative referents), and control beliefs (barriers/facilitators). Two reviewers independently performed qualitative content analysis of interview transcripts to identify salient beliefs in relation to the defined behavior. Results: Out of 150 eligible graduating EM residents, 25 participated in this study. The main reported advantage of contributing to the online slideshow was learning consolidation $(n=15)$; the main reported disadvantage was information overload $(n=3)$. The most frequently reported favorable referents were graduating EM residents writing the certification exam $(n=16)$. Few participants $(n=3)$ perceived any negative referents. The most frequently reported facilitator was the peer-reviewed high-quality scientific information $(n=9)$; the most frequently reported barrier was time constraints $(n=22)$. Conclusion: The beliefs identified in this study will contribute to the design of a theorybased questionnaire to further explore determinants of residents' intentions to contribute to an online collaborative writing project. This will help develop implementation strategies for increasing contributions to other CWAs in medical education.

Keywords: social media, collaborative writing applications, FOAMed

\section{LO39}

The other end: evaluation of blood bank technologists and hematologists during a massive hemorrhage simulation project

A. Levy, MD, MEd, G. Pettersen, MD, MSc, F. Gauvin, MD, A. Sansregret, MD, MAEd, S. Lesage, MD, N. Robitaille, MD, Sainte-Justine Hospital University Centre, Montréal, QC

Introduction: Massive hemorrhages (MH) are life-threatening complications of pediatric trauma and obstetrical cases. Expertise and effective communication is key among all team members actively involved in managing such events. Collaboration with blood bank technologists (BBT) and hematologists responsible in assisting teams with prompt preparation and delivery of blood products further contributes to optimizing care. We aimed to evaluate communication and expertise when preparing and delivering blood products in cases of $\mathrm{MH}$ using in situ simulation. Methods: Prospective observational study held at a blood bank (BB) and simulation lab of a tertiary care facility. Participants were $\mathrm{BBT}$ and hematologists as well as pediatric emergency/intensive care and obstetrical/anesthesia teams submitted to high fidelity (HF) simulated pediatric trauma and post-partum $\mathrm{MH}$ scenarios respectively. $\mathrm{BBT}$ were videotaped at the $\mathrm{BB}$ and conversations with teams at the simlab were recorded. If hematologists were consulted, they were called back by the chief BBT and were asked to answer questions relevant to the application of the MH protocol. All participants were observed initially and two weeks later (post-session). A blinded independent trained rater assessed performances.BBTwere evaluated using a checklist derived by transfusion experts rating expertise and key communication skills necessary when preparing and delivering blood products to teams involved in $\mathrm{MH}$. Hematologists were evaluated using a questionnaire developed by transfusion security experts exploring their ability to assist and communicate with BBT and clinicians when dealing with the choice of blood products or compatibility issues. Means and standard deviations of scores on checklists and questionnaires were calculated. Results: A total of 8 BBT, 8 hematologists and 62 healthcare professionals involved in 8 interdisciplinary teams (4 obstetrics/anaesthesia and 4 paediatric emergency/intensive care) participated in the study. BBT scored on average $78 \%(61-92 \%)$ and $76 \%(62$ to $100 \%$ ) in expertise and communication skills checklists during trauma and postpartum simulations, respectively. Hematologists rightly refused blood specimens to determine blood type in 57\%(4/7) of the cases (discrepant $\mathrm{ABO} / \mathrm{Rh}$ blood group with previous $\mathrm{ABO}$ group results). Their ability to choose blood products or to substitute them was observed in $71 \%(5 / 7)$ of participants. When challenged by the fact that an incompatible blood product was delivered to the bleeding patient, only $37.5 \%(3 / 8)$ asked for phenotype analysis of products already transfused and those being prepared, and no one advised the team of this fact and of the possibility of a haemolytic transfusion reaction. Conclusion: BBT were considered to be relatively well prepared and possess the necessary expertise and communication skills to prepare and deliver blood products to obstetrical and paediatric trauma teams dealing with MH. For hematologists, knowledge gaps were identified and additional training is mandatory to ensure proficiency when assisting teams dealing with such emergencies. Using in situ simulation at the BB and haematologists in addition to HF interdisciplinary team simulations occurring in a simlab can further contribute to improving performances of all professionals actively involved in $\mathrm{MH}$ crisis situations.

Keywords: in situ simulation, education, massive haemorrhages

\section{LO40}

Structured teaching shifts in the emergency medicine clinical clerkship: impact on ED length of stay

L. Shepherd, MD, E. Zibrowski, MSc, M. Klingel, MSc, L. Lingard, $\mathrm{PhD}$, University of Western Ontario, London, ON 
Introduction: The Emergency Department (ED) offers medical students the educational richness of a spectrum of patient presentations. However, there is constant tension between patient flow and learner needs in this clinical education context. A recent national position statement on ED overcrowding suggests that academic centres consider utilization of teaching practices that benefit both learner and department-centred outcomes. This study asked: how do structured 'teaching shifts', which allow clinical clerks and teachers to interact without the pressure of patient care, impact ED patient flow, student satisfaction and student confidence in their knowledge and skills? Methods: In 2012-13, 131 clinical clerks at a single Canadian medical school, participated in 3 teaching shifts at the start of their mandatory emergency medicine rotation. They were involved in a combination of teaching modalities supervised by one faculty member without patient care responsibilities. Patient flow, as measured by ED length of stay (EDLOS) and time to physician initial assessment (PIA), was compared for the three teaching shift days and for the same three days of the week occurring immediately after the teaching shift. Rotation evaluations were compared, before and after implementation to assess student satisfaction. Students' self-assessed knowledge and skills were probed with a questionnaire pre and post teaching shifts. Results: Across 40,998 patient visits, EDLOS was shortened overall by a median of 5 minutes (IQR: 1.2-8.8) and in the first quarter, EDLOS was reduced by 20 minutes (IQR: 12.7-27.3) when clerks were involved in their teaching shifts. PIA time was 5 minutes longer (IQR: 2.2-7.8) during this same period. Rotation evaluations remained high. Self-assessment scores showed statistically significant improvement in knowledge and skills across all twenty items $(p<.001)$. Conclusion: Teaching shifts are an effective educational intervention that positively impact EDLOS and retain a high level of student satisfaction. Using teaching shifts with the most naïve clerks, in the first quarter of the academic year, appears to be of most benefit to EDLOS. While students' confidence in their knowledge and skills improved post teaching shifts, objective measures of student competence are required in future research.

Keywords: education, length of stay, medical student

\section{LO41}

The use of online educational resources by Canadian emergency medicine residents and program directors

E. Purdy, BHSc, J.M. Bednarczyk, MD, D. Migneault, MDCM., B. Thoma, MD, MA, J. Sherbino, MD, MEd, Queen's University, Kingston, ON

Introduction: Online educational resources (OERs) including podcasts, blogs, and social media are increasingly available for emergency medical education. This survey quantified the use of OERs by Royal College emergency medicine (EM) residents and program directors (PDs) and investigated the relationship between using OERs and reading peer-reviewed literature. Methods: An online bilingual survey was designed by authors from five Canadian universities, piloted on a test group, and distributed to Royal College EM residents and PDs using a modified Dillman method. Data were analyzed using SPSS. Results: $214 / 350$ residents $(61.1 \%)$ and $11 / 14$ PDs (79\%) completed the survey. OERs were used by residents most frequently for studying core EM topics $(99.5 \%)$, procedural techniques training $(96 \%)$, and learning diagnostic test and imaging interpretive skills $(92 \%)$. OER modalities used most frequently included wikis $(94.9 \%)$, filesharing sites $(94.9 \%)$, e-textbooks $(93.5 \%)$ and podcasts $(91.1 \%)$. Few residents $(7.5 \%)$ participated in the creation of OERs. Residents used wikis $(p<0.01)$, podcasts $(p<0.01)$, vodcasts $(p=0.02)$ and file sharing sites $(p<0.01)$ significantly more frequently than PDs. However, there were no significant differences in the use of Twitter, Facebook, Google+, e-textbooks, video-sharing sites, blogs or online question repositories. Relative to PDs, residents found 'entertainment factor' $(p<0.01)$ to be significantly more important for choosing OERs. However, when selecting OERs, residents and PDs did not differ significantly in the importance they placed on identifiable authorship, references, use of evidence-based principles, and ease of access. Both groups reported that OERs increased the amount of peer-reviewed literature ( $75 \%$ vs $60 \%$, $\mathrm{p}=0.31$ ) that they read. Conclusion: EM residents use OERs for core EM education, procedural training and learning interpretive skills. Residents value entertainment in OERs and access certain resources more frequently than their PDs. OERs appear to increase the use of peerreviewed literature for both residents and PDs.

Keywords: medical education, online educational resources

\section{LO42}

Quantifying competency - the development and validation of a hand motion analysis program to discriminate experts and nonexperts during central venous line cannulation

D. Clinkard, MSc, M. Holden, M.Eng, D.W. Messenger, MD, G. Fichtinger, PhD, T. Ungii, MD, PhD, C. Davison, PhD, R. McGraw, MD, Queen's University, Kingston, ON

Introduction: Introduction: Traditionally, technical skills proficiency was assessed by direct observation. While direct observation and feedback are essential components in technical skills learning, they demand considerable investment of faculty time. Additionally, as an assessment tool, direct observation is inherently subjective and has been criticized as unreliable. The purpose of this study was to determine if quantitative electromagnetic motion tracking is feasible and could discriminate between experts and non-experts during simulated ultrasound guided insertion of a central venous catheter guidewire. Methods: Methods: 10 resident physicians (FRCP PY1 and 2, CCFP-EM 1) and 10 staff (ICU fellows or attending physicians) were recruited. Electromagnetic sensor probes were used to capture hand motion during an ultrasound guided internal jugular cannulation on a standardized manikin. Hand, ultrasound and needle motion was analyzed for the following metrics: total path length, total time, translational movements and rotational movements. Subjects were also videotaped and evaluated using a global rating scale by a blinded expert. Results: Results: There was a significant difference in almost all examined motion parameters between experts and non-experts. Experts took $66 \%$ less time $(50.2 \mathrm{sec}$ vs. $148.7 \mathrm{sec}$, $\mathrm{p}<0.005$ ) and had significantly less right hand and ultrasound motions (total path, translational and rotational movements). Left hand path distance was the only parameter that was not significantly different between groups (experts $2466.9 \mathrm{~mm}$ vs. non-experts $4120.8 \mathrm{~mm}$, $\mathrm{p}=0.08$ ). Concurrent validity of motion parameters was established by strong correlations ( $r>0.74)$ to a previously published, modified global rating scale. Conclusion: Conclusion: We have demonstrated that electromagnetic hand and instrument motion analysis is technically feasible for assessing competence in the skills of ultrasound guided insertion of a central venous catheter guidewire in a simulated setting. In showing that is discriminates between the performances of non-experts and experts, we have provided evidence for construct validity. It also shows excellent correlation with a modified version of a previously validated global rating scale: evidence of concurrent validity.

Keywords: competency, medical education, evaluation

\section{LO43}

Evaluating and enhancing $M \& M$ rounds aptitudes of residents using the Ottawa M\&M Model (OM3)

S.E. Mondoux, MD, BEng, J.R. Frank, MD, E.S. Kwok, MD, A. Cwinn, MD, L.A. Calder, MD, MSc, Department of Emergency Medicine, University of Ottawa, Ottawa, ON 
Introduction: There is little evidence to support best practices for M\&M rounds despite the fact that the medical profession has long made use of such rounds as an opportunity to inform practice. The Ottawa M\&M Model has been designed to be a more effective approach, but it is not known if it is useful as an educational tool for physicians in training. We studied the effect of a single educational intervention on emergency medicine residents' aptitudes in selecting and analyzing M\&M cases. Methods: In this before and after study conducted with residents of the Royal College Emergency Medicine program at the University of Ottawa, we invited all residents to attend a 1 hour educational session based on the previously published Ottawa M\&M Model (OM3) protocol. Residents were asked to submit an $\mathrm{M} \& \mathrm{M}$ case both pre and post-intervention. A novel M\&M case critique tool was developed based on a previously validated M\&M model and used to assign a numerical score to each submitted case. Our primary outcome was an increase in median effectiveness scores between pre and post-intervention M\&M case submissions using the case critique tool. Results: A total of 19 residents were recruited for our preintervention and 16 residents for the post-intervention analysis. The time elapsed between case critiques was 6 weeks. Data was analyzed using the Mann-Whitney test. The cumulative case critique score interquartile range (IQR) and median was 3.0 and 4.0 respectively preintervention as well as 5.0 and 7.5 respectively post-intervention $(\mathrm{U}=$ $65, \mathrm{Z}=-2.86, \mathrm{P}=0.004)$. Residents chose more clinically common cases with pre and post medians and IQRs of 3.0 and 1.0 and increased to 4.0 and 0.25 respectively $(\mathrm{U}=54, \mathrm{Z}=-3.22, \mathrm{P}=0.001)$. Conclusion: After a simple 1-hour educational intervention using the OM3 protocol, Emergency Medicine residents performed significantly better M\&M case analyses and chose more appropriate M\&M cases.

Keywords: medical education, patient safety

LO44

Performance of a competency-based resuscitation assessment tool (QSAT) - a Canadian multi-centered study

J. Dagnone, MD, MMEd, A.K. Hall, MD, K.G. Woolfrey, MD, C. Davison, PhD, J.R. Ross, MD, G. McNeil, MD, S. Moore, MD, W. Pickett, PhD, Queen's University, Kingston, ON

Introduction: The use of high-fidelity simulation is emerging as an effective method for competency-based assessment in postgraduate medical education. We aimed to validate the Queen's Simulation Assessment Tool (QSAT) in a multi-center setting utilizing simulationbased resuscitation Objective Structured Clinical Examination (OSCE) stations to assess post-graduate emergency medicine trainee competency. Methods: Emergency Medicine postgraduate trainees (FRCP \& CCFP-EM) from five Canadian centres participated in a high fidelity simulation-based resuscitation OSCE. All trainees completed the following three OSCE stations: acute pulmonary edema, subarachnoid haemorrhage, and sympathomimetic toxidrome. An expert panel of emergency physicians from each centre evaluated all trainee performances using the QSAT assessment tool. The QSAT is comprised of four domain-specific anchored scores (i.e. primary assessment, diagnostic work-up, therapeutic actions, and communication) and one global assessment score. The expert panel of Emergency Medicine physicians was blinded to the residents' identity and level of training for all QSAT scoring. Assessment correlation and analysis of variance were calculated. Results: Emergency Medicine postgraduate trainees (FRCP \& CCFP-EM) from Queen's University, Ottawa University, Dalhousie University, the University of Calgary, and the University of Toronto ( $\mathrm{N}=99$ per OSCE station) participated in the study. Inter-rater reliability showed substantial agreement for the total domain specific scores across all scenarios (mean Spearman's rho $=0.75[0.71-0.82]$ ), and moderate agreement for the global assessment score across all scenarios (mean Spearman's rho $=0.69$ [0.63-0.73]. Discriminatory valid- ity was very strong, with Senior FRCP-EM residents outperforming Junior FRCP-EM and CCFP-EM residents in all 3 scenarios $(p=<0.0001)$. Conclusion: This study demonstrates the performance of the QSAT in a multi-center 3-station OSCE using high-fidelity mannequins for the assessment of resuscitation competence in post-graduate emergency medicine trainees. The assessment tool demonstrates strong inter-rater reliability and impressive discriminatory capabilities. Future competency-based assessment for Emergency Medicine trainees should focus on similar methodology.

Keywords: education, simulation, assessment

\section{LO45}

The role of a physician-in-triage (PIT) in enhancing ED flow for CTAS 2 and 3 patients: A community hospital experience

M. Tambakis, MD, S.L. McLeod, MSc, M. Klingel, MSc, N. Sbrocca, BSc, D. Adekoya, MD, S. Ninkovich, MD, Windsor Regional Hospital, Windsor, ON

Introduction: Overcrowding and lengthy emergency department (ED) wait times are challenges facing many communities. One recent strategy to reduce ED wait times has focused on decreasing the amount of time patients spend waiting for an initial assessment by an emergency physician. The objective of this study was to evaluate the effect on patient throughput after implementing a Physician-In-Triage (PIT) model in a community hospital ED. Methods: This was a retrospective review of all patients seen in a community hospital ED (annual census $60,000)$ over two 3 -month periods. Time to physician initial assessment (PIA), ED length of stay (LOS), and the proportion of patients who left without being seen (LWBS) and left against medical advice (LAMA) were compared over a 3-month (Sept-Nov 2011) period where there was no PIT, to a 3-month period (Sept-Nov 2012) when PIT was implemented. PIT was deployed for 4 hours daily between $1300-1500$ and $1930-2130$ to initiate patient management specifically for Canadian Triage and Acuity Scale (CTAS) Score 2 and 3 patients. Results: 34,306 patient encounters were included. Of the 17,484 patients included in the PIT months, 1285 (7.3\%) were seen by a PIT. Patient demographics and acuity before and after PIT implementation were similar. Median PIA time was significantly reduced during the PIT months compared to the control period (92 $\mathrm{min}$ vs $110 \mathrm{~min}$; -18 min, 95\% CI -20.8, -15.2). Median ED LOS was shorter during the PIT period (180 min vs $199 \mathrm{~min}-19 \mathrm{~min}, 95 \%$ CI $-22.8,-15.2$ ). The proportion of patients who LWBS decreased from $6.5 \%$ to $4.7 \%$ ( $1.8 \%$, $95 \%$ CI: $1.3,2.3$ ) and LAMA decreased from $0.5 \%$ to $0.3 \%$ ( $0.3 \%$, $95 \%$ CI: 0.1, 0.4) after PIT implementation. For CTAS 2 and 3 patients only, median PIA time decreased from $118 \mathrm{~min}$ to $103 \mathrm{~min}$ ( $-15 \mathrm{~min}$, $95 \%$ CI: $-18.5,-11.5$ ) and ED LOS decreased from $234 \mathrm{~min}$ to $225 \mathrm{~min}$ after PIT implementation ( -9 min, 95\% CI: -13.9, -4.1). Conclusion: The addition of an emergency physician at triage was associated with an overall decrease in median PIA time, ED LOS and a lower proportion of patients who LWBS and LAMA. Institution of a Physician-InTriage (PIT) may help initiate the initial assessment and ED throughput for patients presenting to a community hospital.

Keywords: triage, physician

\section{LO46}

Regional ED response to severe overcrowding - why diversion strategies are problematic

E. Grafstein, MD, M. de Moor, BSc, MA, M. Stewart, R. Stenstrom, MD, PhD, C. Startup, MA, Vancouver Coastal Health - Providence Health Care, Vancouver, BC

Introduction: We sought to understand what happens to EDs in a regional system when critical total patient volumes exist. Methods: This was a prospective study using administrative ED data at the 5 
largest EDs in Vancouver Coastal Health urban region. A priori we defined a critical "red surge" value as the total volume in the ED equal to or greater than the $99 \%$ ile for total ED volume measured by site in fiscal 2012-13. Sites validated that their ED was stressed when this value was reached. Real time dashboards, updated at 5 minute intervals, were placed in each ED. There was a visual notification to the EDs and the ambulance dispatch when the surge value had been reached. We had a three month (April - July 2013) observation period where no action was taken by ambulance. A log of all alerts, time of surge onset and duration is also captured. This data was analyzed using descriptive statistics. Results: A total of 174 surges occurred on 51/91 study days. There was a total of 212 hours of surge out of 9828 total hours $(2.2 \%$ of the time spent on surge). There were 17 days (33\% of the total number of surge days) where multiple EDs had red surges at the same time on the same day. 9/17 multiple site surges occurred either on a Monday or Tuesday. No multiple site surges occurred on a Saturday or Sunday. $111(64 \%)$ of the onset of surges occurred during the afternoon quartile (12:00 - 18:00) and $47(27 \%)$ occurred in the evening quartile (18:00 - 23:00). Surge duration varied from $10 \mathrm{~min}$ to 740 minutes (median $34.5 \mathrm{~min}$, IQR 15.3 - $83.5 \mathrm{~min}$ ). Overall there were $2500 \mathrm{ED}$ visits during red surge activity at the 5 sites. Breakdown by acuity is as follows: CTAS 1-3 $1471(58.8 \%)$, CTAS 4-5 1017 (40.7\%), and unknown 12 (0.5\%). 533/2500 visits (21.3\%) arrived by ambulance, 1905 (76.2\%) were self-directed and $61(2.5 \%)$ had invalid data. 530 of all patients seen during surge were admitted (21.2\%). In comparison, total ED visits to the region during this 3 month period were 85,408 with 14,484 admissions (16.9\%). There were 16,890 ambulance carries $(19.8 \%)$. The difference between admission rates during surge time compared to non-surge times is significant (X2 $30.6 ; p<0.001)$. There was no statistical difference in the percent of CTAS 1-3 patients seen during surge and non-surge periods. Conclusion: Regional total ED volume surge activity is worse on Mondays/Tuesdays. ED overcrowding may increase the likelihood of admission. Public notification of ED surge may be helpful to influence walk-in diversion strategies.

Keywords: ED overcrowding, volume surge, dashboard

\section{LO47}

A collaborative tale of quality and quantity: enabling emergency physicians to order CT scans without mandating discussion with medical imaging did not increase $\mathrm{CT}$ ultization

D. MacKinnon, MD, M. McGowan, MHK, A. Cheng, MD MBA, T. Dowdell, MD, D. King, MBA MRT(N), G. Bandiera, MD, MEd, University of Toronto, Toronto, ON

Introduction: Many institutions require that $\mathrm{CT}$ requests from emergency physicians (EPs) be approved by medical imaging (MI) as a means to control utilization and organize workflow. However, this may contribute to delay to diagnosis, treatment, and increased emergency department (ED) length of stay. We sought to determine if there was a change in the number or rate of CT scans ordered if EPs were able to order CT scans without mandating discussion with MI. Methods: In 2010, ED and MI administrators in an academic, tertiary-care centre implemented a policy that enabled EPs to order CTs without discussion with MI on Monday to Friday 8am - 5pm (M-F 8-5). There was no change in practice for other time periods. The number of ED CTs performed for 1-year prior and after implementation was reviewed. Results: There was a decrease in the number of ED CT scans performed M-F 8-5 after implementing the policy (2237 pre vs. 2095 post, $6.3 \%$ decrease). There was also a decrease in after-hours ( 4578 pre vs. 4523 post, $1.2 \%$ decrease) and total CTs performed (6815 pre vs. 6618 post, $2.9 \%$ decrease). The decrease occurred despite increases in patient volume, resulting in a lower rate of CTs per ED patient $(0.11$ CTs per patient pre vs. 0.10 post). Conclusion: Enabling EPs to order
CTs without discussion with MI did not significantly affect the number or rate of ED CTs performed. Other EDs can consider this policy as a patient flow initiative with appropriate monitoring of CT ordering rates.

Keywords: patient flow, quality improvement, CT scan

\section{LO48}

Does increasing age affect emergency physician willingness to work night shifts?

P.R. Atkinson, MD, C. Vaillancourt, MD, J. Talbot, MD, M. Howlett, MD, Dalhousie University, St. John, NB

Introduction: In several industries, there is established evidence of the physiological toll of night shifts on older workers. We wished to assess if such pressures translated into actions by older emergency physicians (EPs) resulting in them working fewer night shifts in a tertiary urban emergency department (ED). Unless excluded from nights for medical reasons, or having requested more night shifts by personal preference, all EPs are allocated a proportional number of nights according to the total number of weekly shifts they work. Night shifts are paid at a preferential rate ( $20 \%$ higher hourly rate than day and evening shifts). Shifts can be traded by mutual agreement. EPs may choose to work full time (more than 3 shifts per week) or part time. Methods: We compared choices to work full or part time, mean number of night shifts worked, and scheduled versus actual night shifts worked for a group of $23 \mathrm{EPs}, 12$ of whom were classified as younger (under 40 years) and 11 who were classified as older (over 40 years). One outlier who worked night shifts preferentially and who accounted for around one third of all night shifts was excluded from the analysis of night shifts. Data was analyzed using GraphPad software. Results: Older EPs were more likely to work part time than were younger EPs $(9 / 11$ vs $11 / 13 ; \mathrm{p}=0.0031$; Fishers exact test). Older EPs worked fewer night shifts than younger EPs (annual mean of 5.6 vs 11.7; $\mathrm{p}=0.021$; Student $\mathrm{T}$ test). There was a non-significant trend for older EPs to reduce their scheduled number of night shifts from $43.5 \%$ (95\% CI 37.3-49.8\%) to $39.2 \%$ (33.2 - 45.6\%) nights actually worked, whereas younger EPs (under 40 years) increased their number of scheduled night shifts from $56.5 \%(50.2-62.7 \%)$ to $60.8 \%(54.4-66.8 \%)$. Conclusion: Even without a formal night shift policy in place, we found that that older EPs were more likely to work part time clinical emergency shifts supplemented by other clinical, administrative and academic work and work fewer night shifts annually. There was also a weak trend towards older EPs relinquishing their night shifts in this tertiary level ED. We hope to survey similar EDs regarding night shift policy, and plan to develop an evidence based night work schedule locally.

Keywords: career, work patterns, emergency medicine

\section{LO49}

Emergency department visits after diagnosed chronic obstructive pulmonary disease in aboriginal peoples in Alberta

M.B. Ospina, PhD, D. Voaklander, PhD, A. Senthilselvan, PhD, M.K. Stickland, PhD, M. King, PhD, B.H. Rowe, MD, MSc, University of Alberta, Edmonton, AB

Introduction: Chronic obstructive pulmonary disease (COPD) often requires frequent visits to the emergency department (ED). Access to ED services for COPD is an important determinant of respiratory health that has the potential to contribute to health inequalities between Aboriginal peoples and the rest of the Canadian population. This retrospective cohort study compared the patterns of ED visits following a COPD diagnosis in Aboriginal peoples relative to a non-Aboriginal population in Alberta. Methods: Linked administrative health services data from Alberta were used to identify Aboriginal peoples and nonAboriginals who were diagnosed with COPD between 2002 and 2009. 
Rate ratios (RR) and 95\% confidence intervals (CI) of all-cause ED visits and for ED visits due to acute exacerbations of COPD (AECOPD) were assessed using Poisson models adjusting for important socioeconomic and clinical factors. Results: A total of 2,274 Aboriginal peoples were newly diagnosed with COPD during the study period. All-cause ED visit rates given COPD were significantly higher among Aboriginal peoples compared to the non-Aboriginal group (RR $=1.72,95 \%$ CI: 1.67, 1.77). Similarly, ED visit rates for AECOPD in Aboriginal peoples were significantly higher than those of non-Aboriginals $(\mathrm{RR}=1.33 ; 95 \% \mathrm{CI}: 1.21,1.45)$. Conclusion: Aboriginal peoples with $\mathrm{COPD}$, as a whole group, had significantly higher rates of allcause and AECOPD ED visits compared to non-Aboriginals with COPD. Further research is needed on how access barriers for primary care services contribute to these patterns of ED use among Indigenous peoples.

Keywords: aboriginal peoples, COPD, frequent emergency department users

\section{LO50}

Predictors of awareness and use of an emergency department wait times website in three Canadian urban settings

B. Voaklander, A. Davidson, M.B. Ospina, PhD, S.W. Kirkland, MSc, R. Chetram, G. Cummings, MD, T. Nikel, B. Holroyd, E. Cross, C. Villa-Roel, S. Couperthwaite, BSc, D. Voaklander, PhD, B.H. Rowe, MD, MSc, University of Alberta, Edmonton, AB

Introduction: Alberta Health Services (AHS) displays wait times on its website for the main urban emergency departments (ED). This study analyzed factors associated with awareness and use of this online tool by patients presenting to three urban EDs in Edmonton, Alberta. Methods: Patients $>17$ years of age were randomly selected from electronic registration records at three urban ED sites and asked to complete a questionnaire. Associations among sociodemographic factors and awareness and use of the ED wait times website were explored using backward logistic regression models. Results: Overall, 1408 patients completed the survey; $22.4 \%$ were aware of the ED wait times website and $6.8 \%$ checked the website prior to their visit. Females (OR $=1.4 ; 95 \%$ CI: $1.07,1.9)$, individuals with higher education $(\mathrm{OR}=1.7$; $95 \%$ CI: $1.3,2.2)$, and those with a family physician (OR $=1.9 ; 95 \%$ CI: $1.3,2.7$ ) were more likely to be aware of the website. Being older than 40 years, never married and non-Caucasian were significantly associated with less awareness of the website $(p<0.05)$. Factors significantly related with less use of the website were: being older than 25 years (OR: $0.2 ; 95 \% \mathrm{CI}: 0.1,0.5)$, never married $(\mathrm{OR}=0.7 ; 95 \% \mathrm{CI}$ : $0.5,0.9)$ and non-Caucasian ( $\mathrm{OR}=0.5 ; 95 \% \mathrm{CI}: 0.3,0.9)$. Conclusion: Both awareness and use of a website informing on wait times in urban EDs are low. Factors associated with use and awareness of the website suggest inequity issues that require attention.

Keywords: knowledge translation, waiting times, access to care

LO51

Emergency department patients with amphetamine-related psychosis have high subsequent community resource needs E. Grafstein, MD, B. Daly-Grafstein, BSc, M. Corral, MD, A. Nazif, MD, S. Fitzpatrick, MD, R. Stenstrom, MD, PhD, F.X. Scheuermeyer, MD, St. Paul's Hospital, Vancouver, BC

Introduction: We sought to investigate whether ED patients with amphetamine-related psychosis (ARP) in an urban setting cause a substantial ED resource and social burden in the year following an ED visit. Methods: Consecutive patients presenting to two urban EDs with ARP from 2008-2011 were identified retrospectively via the following explicit criteria: all patients with an ED diagnosis of (1) "amphetamine intoxication" (ARI) with documented amphetamine use plus "disorga- nized thought," "bizarre behavior," or "delusional behavior"; or (2) "psychosis" (PNOS) with a positive urine test for amphetamines, and one or more of the following interventions: police arrival, mental health certification by community or emergency physician, physical restraints applied, or the use of ED parenteral sedation or antipsychotic. Patient demographics, comorbidities, medications, and ED treatment and outcomes were identified via chart review. This cohort was linked with regional data to identify subsequent ED visits, with provincial Vital Statistics to obtain mortality, and with the local police database to identify encounters with the police. The primary outcome was the number of ED visits for the year post ARP encounter. Secondary outcomes included police interactions and arrests for violent crime in the year post ARP encounter. Results: From 2008 to 2011, 326 patients with ARI patients and 1464 patients with PNOS were screened.187 ARP patients (77 ARI and 110 PNOS) patients met the inclusion criteria. The majority $(123 / 187$ [66\%]) were male with median age of 32 (IQR 26, 40), and $72 \%$ were transported to the ED. (34\% police, $38 \%$ ambulance) 103/187 (55\%) were admitted. The median EDLOS for all admitted patients was 42.7 hours (IQR 29.2, 62.7 ) and for discharged patients was 5.1 hours (IQR 2.0 - 10.7). In the year after ARP presentation patients made 868 visits to regional EDs (mean 4.6 visits/patient) of which $35 \%$ (303/868) were mental health related. 86 patients (46\%) required a total of 199 re-admissions to hospital in the following year. 28 patients $(15 \%)$ had a total of 58 police encounters, resulting in arrests for violent incidents. Four patients $(2.2 \%)$ died within 1 year of ARP diagnosis but none within 3 months of the index ARP visit. Conclusion: Conclusion: ED patients with ARP have a disproportionately high resource use at the index ED encounter, and have high rates of subsequent ED recidivism and police involvement. Further research has begun to see the impact of acute Addictions Consultation for this group.

Keywords: amphetamine, psychosis, resource utilization

\section{LO52}

\section{Intoxicated adolescents in the emergency department}

K. Sullivan, MD, R. Grierson, MD, C. Barber, BSc(Hon), M. Tenenbein, MD, E. Weldon, MD, University of Manitoba, Winnipeg, MB

Introduction: Intoxicated adolescents in the Emergency Department (ED) present a clinical and social challenge not well described in the literature. The purpose of this study was to characterize the adolescent population seen, treated, and discharged from the ED for intoxication. Outcomes of interest included ED length of stay, discharge failures, and police and ambulance utilization rates. Methods: A retrospective chart review of adolescent patients presenting with intoxication to an inner city, tertiary care pediatric ED was performed (Winnipeg, Manitoba). Patients with alcohol, presumed alcohol, and mixed intoxication with alcohol were included. Once identified, a pre-piloted standardized case report form was used to extract data from the clinical record. All data was collected using trained recorders after a successful calibration exercise. Details of each visit - demographics, triage presentation, physical examination, interventions, discharge disposition, length of stay (defined as time to triage to time to discharge), discharge failures (defined as return visits for intoxication within $24 \mathrm{hrs}$ ), and ambulance and police utilization were analyzed. Select variables were compared to data of an age-matched control group presenting during the same time period. Ten percent of charts were recorded in duplicate and assessed for inter-rater reliability. Categorical variables were compared with the Pearson Chi-square test, and continuous variables were compared via the two-sample t-test. The Fleiss Kappa statistic was used to measure level of agreement between study data abstractors for categorical outcomes. Results: One hundred and fifty-six charts were included in this study. The intoxicated population was predominantly female $(69 \%$ vs. $51 \%$, p $<0.001)$, without a primary care provider $(60 \%)$, and 
had co-ingested alcohol with other substances (54\%). Intoxicated patients arrived more frequently with ambulance or police (74\% vs. $16 \%, \mathrm{p}<0.0001)$, and with more acute CTAS scores than the control population $(\mathrm{p}<0.0001)$. There was a trend toward longer average length of stay for the intoxicated youth group (4 hrs vs. $3 \mathrm{hrs}$ and 26 min, $p=0.06$ ), however discharge rates between the study and control populations were similar ( $84 \%$ and $85 \%$ respectively, $\mathrm{p}=0.9$ ). No discharge failures were reported. Inter-rater agreement was excellent ( = $0.8-1.0$ ) for all but one of the variables, with the category of neurologic exam performed having a kappa value of 0.59. Conclusion: Intoxicated adolescents comprise a vulnerable population. Although not commonly critically ill, or injured, they use significant resources. Despite their vulnerability, medical assessments are often incomplete, and interventions and admissions are uncommon. Programs designed to decrease ED visits, resource utilization, and improve the health of the intoxicated youth population appear warranted.

Keywords: intoxication, adolescent, emergency department

LO53

The use of intralipid and high dose insulin therapy in the emergency department management of beta blocker and calcium channel blocker toxicity

M. Riggan, MD, S.L. McLeod, MSc, H. Hames, MD, University Western Ontario, London, ON

Introduction: Beta-blocker (BB) and calcium channel blockers (CCB) are widely prescribed drugs with a high potential for toxicity when taken inappropriately. Treatment of this toxicity is challenging. Intralipid and high-dose insulin have emerged as new therapies effective in many $\mathrm{BB}$ and $\mathrm{CCB}$ toxicities. The objective of this study was to review the management of $\mathrm{BB}$ and $\mathrm{CCB}$ toxicity by emergency department (ED) physicians with a focus on the use of intralipid and high dose insulin euglycemic therapy (HIE). Methods: This was a retrospective medical record review of adult ( $>17$ years) patients presenting to one of two EDs at an academic tertiary care centre (annual census 140,000) from April 2007 to March 2012 with the presumed or final diagnosis of $\mathrm{BB}$ or $\mathrm{CCB}$ toxicity. Patient demographics, specific drugs ingested, vital signs, investigations and treatments ordered and initiated by ED physicians, contact with poison control, inpatient management and patient outcomes were recorded. Results: Of the 103 charts reviewed, 26 were included. Mean (SD) age was 53.0 (11.7) years and 17 (65.4\%) were male. 20 (76.9\%) patients declared intentional overdose at triage. $16(61.5 \%)$ patients ingested a BB, $8(30.8 \%)$ patients ingested a CCB and $2(7.7 \%)$ patients ingested both. The most common ingestions were Atenolol and Metoprolol. 15 (57.7\%) patients were hypotensive (SBP $<90 \mathrm{mmHg}$ ); 6 patients were hypotensive from presentation and 9 patients decompensated in the ED. 11 hypotensive patients were intentional ingestions. Of hypotensive patients, 7 received decontamination by ED physicians, 5 received glucagon, 8 received calcium, 9 received vasopressors, 5 received HIE and no patients received intralipid. ED physicians contacted Poison control centre for 6 patients, 4 of which were hypotensive. 7 patients went to the ICU; 3 were started on HIE and 2 received intralipid. 2 patients died after being referred to ICU. Both received intralipid and HIE. One died $24 \mathrm{hrs}$ later in the ICU after attempted dialysis. The other died in the ED after failing cardiac bypass. Conclusion: Despite good evidence to support the effectiveness of intralipid and HIE, their uptake has been slow in the ED community. Future research should attempt to identify factors that influence the decision to initiate treatment with HIE or intralipid by ED physicians.

Keywords: overdose, intralipid, high dose insulin

\section{Moderated Posters Presentations}

\section{MP01}

Tourniquet utilization patterns \& impacts on hemostasis in a large, urban EMS system

J.M. Goodloe, MD, M. Martinez, MD, A.O. Arthur, PharmD, S. Parker, S.H. Thomas, MD, MPH, The University of Oklahoma School of Community Medicine, Tulsa, OK

Introduction: Combat casualty care education and peer-reviewed manuscripts describing this care as performed in the military conflict theater indicate significant survival benefits from the timely application of tourniquets in the setting of extremity trauma with significant ongoing hemorrhage. There is a paucity of published clinical data regarding the translation of this knowledge into civilian use by Emergency Medical Services (EMS) systems. This study's purpose was to analyze a civilian patient cohort in which tourniquets were utilized as hemostatic intervention in a large, urban EMS system in the southwestern United States. Methods: Retrospective chart review of consecutive patient encounters involving use of at least one tourniquet occurring August 1, 2010 to June 1, 2013 in the study EMS system. Results: In the 34 month study period, 106 patient encounters involved application of at least one tourniquet. 83/106 (78.3\%) of patients were male. Mean patient age was 40.4 years old, with $10 \%$ of patients being 65 years of age or older. The most common injury type was laceration, sustained in $62 / 106(58.5 \%)$ patients. The second most common indication for tourniquet application was bleeding from a dialysis fistula in 14/106 (13.2\%) patients. 80/106 (75.5\%) injuries treated were located on an upper extremity, and 74/106 (69.8\%) injuries were located in the proximal half of the involved limb(s). In the 80 patients in which pre-tourniquet hemostatic treatment was attempted, the most common option failing to achieve desired hemostasis was direct pressure, utilized in $58 / 80(72.5 \%)$ of this subgroup. Overall, hemostasis was documented post-tourniquet application as successful in 90/106 (84.9\%) patients. Using Fisher's exact test, there was not a statistically significant difference in achieving hemostasis comparing upper to lower extremity location $(\mathrm{p}=0.36)$ or comparing distal to proximal injury location $(\mathrm{p}=$ $0.50)$. Conclusion: In a civilian cohort of patients with extremity injuries treated with tourniquets applied by EMS professionals in a large, urban EMS system, hemostasis was achieved in the large majority. Location of injury and limb(s) involved did not affect likelihood of resulting hemostasis.

Keywords: emergency medical services, trauma, hemorrhage control

\section{MP02}

Emergency medical services provider comfort with pre-hospital analgesia administration to children

B. DeBruyne, MD, A. Rahman, MD, S.J. Curtis, MD, MSc, S. Sookram, MD, S. Ali, MDCM, Department of Pediatrics, University of Alberta, Edmonton, AB

Introduction: The under-treatment of pediatric pain is a significant concern among emergency clinicians and researchers. Although some have examined pre-hospital pain management, the deterrents to pediatric analgesia administration by Emergency Medical Services (EMS) have not yet been examined in Canada. This study was conducted to describe EMS pain management practices and provider comfort treating pediatric pain, describe differences in pain management between adults, adolescents and children, and to assess the potential barriers, misconceptions, difficulties and needs related to successful provision of pediatric analgesia. Methods: A study-specific survey tool was created and distributed to all primary care paramedics (PCPs) and advanced care paramedics (ACPs) over four mandatory educational 
seminars in the city of Edmonton from September to December 2008. Results: Ninety-four percent (191/202) of EMS personnel for the city of Edmonton completed the survey. The majority of respondents were male $(73 \%, 139 / 191)$, aged $26-35(42 \%, 80 / 191)$ and had been in practice less than 10 years $(53 \%, 101 / 191)$. Seventy-four percent (141/191) of those surveyed were ACPs, while 26\% (50/191) were PCPs. Although the majority of respondents reported using both pain scales and clinical judgement to assess pain for adults $(85 \%, 162 / 191)$ and adolescents $(86 \%, 165 / 191)$, children were 6 times more likely than adults $(31 \%, 59 / 191$ versus $5 \%, 10 / 191)$ to be assessed by clinical judgement alone. EMS personnel felt significantly more comfortable treating adults than children $(\mathrm{p}<0.001)$, and they were less likely to treat children even if they were experiencing identical types and intensities of pain as adults (all $\mathrm{p}$ values $<0.05$ ) and adolescents (all $\mathrm{p}$ values $<0.05)$ Twenty-five percent of providers (37/147) assumed pediatric patients required less analgesia due to immature nervous systems. The major barriers to treating children's pain included limited clinical experience $(34 \%, 37 / 110)$, difficulty in communication $(24 \%, 26 / 110)$ and inability to assess children's pain accurately $(21 \%, 23 / 110)$. Conclusion: EMS personnel self-report that children's pain is less rigorously measured and treated than for adults. Educational initiatives aimed at increasing clinical exposure to children, as well as further education regarding simple pain measurement tools for use in the field may help to address identified barriers and discomfort with assessing and treating children.

Keywords: pain, paramedic, emergency

MP03

Prehospital STEMI patients not diverted to PCI facility: interventions by ED physicians

A.B. Tanguay, MD, MSc., F. Bégin, MD, D. Hébert, R. Fleet, MD, $\mathrm{PhD}$, Université Laval, Québec, QC

Introduction: The Unité de coordination clinique des services préhospitaliers d'urgence (UCCSPU) is a base hospital providing online medical support to paramedics. An emergency physician (EP), via a telemedicine system, makes diagnosis of ST-segment elevation myocardial infarction (STEMI) remotely and assess for diversion for primary percutaneous coronary intervention (PCI) or transport to the nearest emergency department (ED) in accordance with the American Heart Association/ Canadian Cardiovascular Society (AHA/CCS) guidelines. We studied ED interventions for the STEMI patients not meeting criteria for diversion to PCI facility. Methods: We examined a retrospective cohort of patients with a remote diagnosis of STEMI from July 2006 to December 2012. Part of the data were extracted from the administrative and clinical databases of the UCCSPU by the nurse coordinator, and verified by a EP supervisor. Other data were extracted from hospital records. Results: : Of the 670 prehospital STEMI patients diagnosed remotely, $467(69,7 \%)$ were diverted to a PCI facility and the remaining $203(30,2 \%)$ were transported to the nearest ED for the following reasons: $148(72,9 \%)$ of them did not meet AHA/CCS criteria because of an expected transport time to the PCI facility exceeding 60 minutes, $24(11,8 \%)$ had medical conditions requiring immediate medical interventions in an ED, $11(5,4 \%)$ patients did not have active chest pain despite a positive ECG for STEMI. The remaining $20(9,8 \%)$ patients had either a non-diagnostic ECG, terminal illnesses or met bad weather conditions. The data from hospital records were available for 159 of the 203 STEMI patients not diverted to the PCI facility. Of those, 121 (76\%) had a persistent STEMI in the ED and 44,6\% (54/121) received fibrinolysis. Most of them (49/54) were subsequently transferred to a PCI facility. $37,2 \%$ $(45 / 121)$ did not receive fibrinolysis and were immediately transferred upon arrival from the ED to a PCI facility. Of those 45 patients, 16 had contraindications to fibrinolysis. Finally, $21(17,3 \%)$ out of 121 had no intervention or were given comfort care. Conclusion: Among STEMI patients diagnosed remotely and not diverted to PCI facility, less than one half of them received fibrinolysis in the ED. More than a fourth of those STEMI patients diagnosed remotely and not diverted to PCI facility were subsequently transferred from the ED to a PCI facility despite no contraindication to receive fibrinolysis.

Keywords: pre-hospital, STEMI, fibrinolysis

\section{MP04}

Experiential and rational decision-making: a survey to determine decision-making styles of paramedics and paramedic students

J.L. Jensen, BSc, W. Tavares, L.A. Calder, MD, MSc, A. Bienkowski, BSc, M. Walker, A. Travers, MD, MSc, P. Croskerry, MD, PhD, Dalhousie University, Halifax, NS

Introduction: Two major processes underlie human decision-making: experiential (intuitive) and rational (conscious) thinking. The predominant thinking style that paramedics and paramedic students use for making clinical decisions is unknown. Methods: A cross-sectional survey using the Rational Experiential Inventory-40, a 40-question validated psychometric tool, was administered to ground ambulance paramedics and paramedic students at the end of their studies. Rational and experiential thinking were each evaluated with twenty questions, ten assessing preference toward and ten assessing perceived ability to use that style. Questions were scored on a five-point Likert scale with higher scores indicating affinity for that style. Descriptive statistics and t-tests were used to determine differences in overall thinking style scores, preference towards, and ability to use each style. Results: The response rate was $93.2 \%(1177 / 1263)$. Most working paramedics were male $(\mathrm{n}=628,69.5 \%)$ with median age of 36 years (IQR 29-42) and median years of experience 10 (IQR 4-16). Most paramedic students were male $(\mathrm{n}=179,63.4 \%)$ with median age 23 years (IQR 21-26). Mean overall score for rational thinking was 3.86/5 (95\%CI 3.83-3.87) among working paramedics and 3.97/5 (95\%CI 3.92-4.02) among students $(\mathrm{p}<0.001)$. Mean overall score for experiential thinking was $3.41 / 5$ (95\% CI 3.38-3.44) among working paramedics and 3.35/5 (95\%CI 3.30-3.41) among students $(\mathrm{p}=0.06)$. For ability to use each thinking style, both groups scored rational thinking higher than experiential thinking: working paramedics $3.93 / 5$ vs. $3.60 / 5$ ( $<<0.001$ ); students $4.03 / 5$ vs. $3.55 / 5(p<0.001)$. For preference, both groups preferred rational over experiential thinking: working paramedics 3.79/5 vs. $3.22 / 5(p<0.001)$; students $3.90 / 5$ vs. $3.16 / 5(p<0.001)$. Conclusion: Paramedics and paramedic students prefer and perceive they are able to use rational over experiential thinking, with higher scores found in the student group than the working paramedics. This study adds to what is known on paramedic decision-making and is important for developing education, clinical support tools, and determining how to assess decision-making in training.

Keywords: paramedics, emergency medical services, clinical decision making

\section{MP05}

A comparison of two structured emergency medical dispatch protocols in Ontario

M. Feldman, MD, PhD, J.M. Bryan, MD, L. Turner, PhD, R. Burgess, BSc, Sunnybrook Centre for Prehospital Medicine, Toronto, ON

Introduction: Emergency medical dispatch is the first medical contact for many patients and involves classification and prioritization of emergency calls and assignment of appropriate emergency medical services (EMS) resources. In Ontario, two different structured dispatch interrogation algorithms have been adopted. The Dispatch Priority Card Index version II (DPCI II) was developed by dispatch experts and EMS physicians in Ontario, while the Medical Priority Dispatch Sys- 
tem (MPDS) is a widely used commercial dispatch product. Differences in performance of the algorithms could impact large numbers of patients and we compared the two algorithms to identify whether there are systematic differences in sensitivity, specificity, and accuracy. Methods: DPCI II data were obtained from Peel Region and the County of Simcoe for calls dispatched from July 1, 2009, through December 31, 2009, and MPDS data were obtained for Toronto calls dispatched from March 1, 2003, to February 29, 2004. All dispatch information was linked to the corresponding ambulance call reports to obtain paramedic assessments of patient acuity. We excluded interfacility transfers, duplicate calls, calls with incomplete data, cancelled calls, and patient refusals of transport. We calculated sensitivity (the ability of the dispatch algorithm to detect high acuity when present), specificity (the ability of the dispatch algorithm to rule out high acuity when it is absent), and accuracy (the proportion of correct assessments of acuity) for the two dispatch algorithms. Results: 102582 MPDS calls met inclusion criteria, as did 25895 DPCI II calls. The proportion of calls rated as high acuity by paramedics differed between the two groups $(18.7 \%$ in the MPDS group, $26.8 \%$ in the DPCI II group, $\mathrm{p}<0.0001)$. Data were standardized using the MPDS group CTAS ratings to account for population differences in acuity. MPDS had a sensitivity of $0.64(95 \%$ confidence interval $0.64,0.65)$ while DPCI II had a sensitivity of $0.89(0.89,0.90)$. Specificity of MPDS and DPCI II was $0.71(0.71,0.72)$ and $0.37(0.37,0.38)$, respectively; while accuracy was $70.03(69.12,70.92)$ and $47.17(46.19,48.15)$, respectively. Conclusion: DPCI II had a significantly lower accuracy than MPDS, even after accounting for underlying population differences in illness acuity. Overtriage occurs significantly more often using the DPCI II dispatch protocol, and results increased unnecessary lights and sirens responses and increased EMS resource utilization.

Keywords: emergency medical services, dispatch

\section{MP06}

Paramedic diagnostic accuracy of ST-elevation myocardial infarction on 12-lead ECG: a systematic review

O. Loubani, BSc,MD, J. McVey, MD, B. Deveau, BA, J.L. Jensen, BSc, Y. Leroux, MD, A. Travers, MD, MSc, Nova Scotia Emergency Health Services, Halifax, NS

Introduction: In many EMS systems, physician electrocardiogram (ECG) interpretation is required to diagnose ST-elevation myocardial infarction (STEMI) in the prehospital setting. This requires time for ECG transmission and communication, and may delay time to reperfusion. The objective of this systematic review was to determine accuracy of paramedic interpretation of ECG for STEMI patients in the prehospital setting when compared to physician interpretation. Methods: Diagnostic studies were identified using EMBASE, MEDLINE, CINAHL, the Cochrane Review Group database, clinicaltrials.gov, hand searching bibliographies, and author contact. Studies where STEMI diagnosis by paramedics was compared against diagnoses made by non-paramedics were considered. Two authors conducted independent review for inclusion at the review of title, abstract, and full article stages, with agreement measured with kappa. Disagreement was resolved with third party adjudication. Editorials, opinions, and non-systematic reviews were excluded. Diagnostic accuracy (sensitivity, specificity, and likelihood ratios) was abstracted and reported. Results: Our search identified a total of 4,897 references, of which 21 met final inclusion. Inter-rater agreement for review of title, abstract, and full papers was 0.65 (95\% CI $0.62-0.68), 0.53$ (95\% CI $0.47-0.59$ ), and 0.89 (95\% CI $0.82-0.97$ ), respectively. In the included studies, there were a total of 4,784 separate ECGs read by paramedics, with 3,015 ECGs interpreted in a real life scenario, and 1,769 interpreted in a simulation setting. The pooled sensitivity and specificity of all trials was $92.1 \%$ (95\% CI 90-94.2\%), and 94.7\% (95\% CI 93.4-96.0\%) respectively, with a positive likelihood ratio of 17.4 and a negative likelihood ratio of 0.083 . There was great variability among studies in the level of training of paramedics for ECG interpretation of STEMI. Conclusion: Paramedics are able to interpret ECGs for the diagnosis of STEMI with a high degree of sensitivity and specificity in both simulation and real world settings. Paramedic diagnosis of STEMI on ECG greatly increases the likelihood of the presence of STEMI. Further investigation is required to determine if paramedic diagnosis of STEMI on ECG has an impact on time to definitive therapy or patient outcomes.

Keywords: STEMI, paramedic, EKG/ECG

\section{MP07}

Prehospital nitroglycerin in tachycardic chest pain patients: risky or not?

M. Proulx, MSc, D. Ross, MD, C. Vacon, PhD, L. Juste, MA, L. de Montigny, PhD, E. Segal, MD, Urgences-santé, QC

Introduction: Tachycardia $(>100 \mathrm{bpm})$ is a contraindication for nitroglycerin (NTG) administration in the 2010 American Heart Association (AHA) Guidelines, despite limited evidence. We sought to determine whether NTG administration for chest pain is more likely to cause hypotension (systolic blood pressure $<90 \mathrm{mmHg}$ ) in patients with tachycardia compared to patients without tachycardia $(50<\mathrm{HR}$ $\leq 100$ ). Methods: We performed a retrospective chart review of primary care paramedic $(\mathrm{PCP})$ run sheets during three years preceding AHA guideline implementation. Using logistic regression, we calculated odds ratios (OR) and 95\% confidence intervals (CI) for the association between NTG-induced hypotension and tachycardia independent of age, sex and four comorbidities (CAD, diabetes, hypertension and dyslipidemia). Results: The cohort included 10,414 consecutive prehospital patients administered NTG; 2079 (20\%) patients were tachycardic before NTG administration. Hypotension occurred in 331 (3.2\%) patients: in $249(3 \%)$ without tachycardia and $82(3.9 \%)$ with tachycardia. Tachycardic patients showed increased adjusted odds of hypotension when compared to non-tachycardic patients (OR 1.28; 95\%CI:1.16-1.42). Hypotension was not associated with age, sex or comorbidities. Conclusion: The absolute risk of NTG-induced hypotension was low. There was a statistically significant increase in the relative risk of hypotension with NTG administration in patients who were tachycardic. EMS medical directors reviewing PCP chest pain protocols should weigh the potential benefits of NTG administration against its known risks.

Keywords: pre-hospital care, tachycardia, nitroglycerin

\section{MP08}

First-responder accuracy using SALT after brief initial training C. Lee, MD, S.L. McLeod, MSc, M. Klingel, MSc, M. Peddle, MD University of Western Ontario, London, ON

Introduction: Mass-casualty incidents (MCIs) present a unique challenge in terms of triage and response for the medical community. The Centers for Disease Control and Prevention in the United States recently proposed a mass-casualty triage tool entitled SALT (sort, assess, life-saving interventions, treatment and/or transport) to equip responders with a consensus triage strategy. Paramedics and emergency physicians have been shown to accurately apply SALT, however first responders to MCIs may also include police and fire services with varying levels of medical training. The objective of this study was to determine if students enrolled in paramedic, fire, and police training programs could accurately apply SALT. Methods: All students enrolled in the primary care paramedic (PCP), fire, and police foundation programs at two colleges were invited to participate in a 30minute didactic session on SALT. Immediately following this session, a 17-item test was administered to assess the student's ability to under- 
stand and apply SALT. General linear models with repeated measures were used where appropriate and chi square tests were used to compare the proportion of errors made amongst respondents. Results: 465 trainees completed the test. The mean (SD) test score for all respondents was $75.0 \%$ (15.9). Test scores were higher for PCPs $(87.0 \%)$ compared to Fire $(80.2 \%)$ and Police $(68.0 \%)$. Fire trainees scored significantly higher compared to Police trainees ( $12.2 \%$; 95\% CI: 8.3 , 16.2). When responders were asked to assign triage categories to 10 paper-based clinical scenarios, over-triage errors occurred in $13.5 \%$ of responses, compared to under-triage in $8.5 \%$. Critical errors, defined as erroneous triage resulting in irrevocable detriment to patient morbidity or mortality, were rare, occurring in $0.8 \%, 1.7 \%$, and $4.9 \%$ of PCP, Fire, and Police responses, respectively. Conclusion: Amongst firstresponder trainees, PCPs are able to apply SALT with the most accuracy, followed by Fire and then Police. Over-triage is the most frequent error, while critical errors are rare. Standardizing SALT across agencies and jurisdictions may lead to decreased confusion, more efficient and accurate pre-hospital care, and improve destination decision-making for first-responders involved in a MCI.

Keywords: emergency medical services, mass casualty incident

\section{MP09}

Factors that predict pre-hospital paramedic IV cannulation success: a retrospective analysis

T. Walker, MD, M. Klingel, MSc, S.L. McLeod, MSc, S. Romano, HBSc, A. Ahmed, BSc, A. Dukelow, MD, Southwest Ontario Regional Base Hospital Program, London, ON

Introduction: Intravenous (IV) cannulation is an enhanced paramedic skill required for the administration of IV medications and fluids in the pre-hospital setting. Despite this, IV proficiency is variable amongst providers and the factors contributing to IV success have yet to be defined. The objective of this study was to determine paramedic factors associated with successful IV cannulation in the pre-hospital environment. Methods: This was a retrospective review of data gathered from 6 emergency medical services from a Regional Base Hospital Program from April 2011 to March 2012. Paramedics not certified in IV cannulation and those who attempted less than 3 IV cannulations were excluded. IV success was defined as successfully catheterizing a patient's vein in $75 \%$ of the attempts made over the study period. Backwards stepwise multivariable logistic regression models determined predictor variables independently associated with successful IV cannulation in the pre-hospital setting. Results: 353 paramedics performed a total of 12,728 IV attempts over the 1-year study period. $85(24.1 \%)$ were advanced care paramedics (ACPs) and 268 $(75.9 \%)$ were primary care paramedics. $271(76.8 \%)$ were full time employees. Paramedic training level, years since IV certification, call volume, error rate, number of IV attempts, proportion of high acuity calls, proportion of older patient ( $\geq 75$ years) calls, and the proportion of calls in an urban setting were variables included in the adjusted model. ACP certification (OR: $3.1,95 \%$ CI: $1.7,5.5$ ) and IV attempts $\geq 40$ the (OR: 2.0, 95\% CI: 1.1, 3.4) were independently associated with IV success. Conclusion: Two paramedic factors were independently associated with successful IV placement. These factors should be considered when developing training benchmarks for skill development and maintenance.

Keywords: emergency medical services, education, IV cannulation

\section{MP10}

Impact of the rapid assessment zone on emergency department throughput and quality measurements

M. Lecavalier, MD, A. Cheng, R. Stenstrom, MD, PhD, St Paul's Hospital, Vancouver, BC
Introduction: Waiting room medicine or assessment of Emergency Department (ED) patients who cannot be assigned to a standard care space (monitored bed) has been a reality for many years in Canadian EDs. In 2008, St Paul's Hospital ED implemented a Rapid Assessment Zone (RAZ) where patients are assessed and then returned to the waiting room. Our objective is to evaluate the impact of the RAZ on measures of throughput and quality for CTAS 2 to 5 patients. We hypothesized that time to physician assessment (TPA), ED length of stay (LOS) and left without being seen (LWBS) rates would decrease in the 3 years after compared to the three years before the implementation of the RAZ, and that adverse outcomes (unscheduled return and admission) within 72 hours would not increase. Methods: This is an administrative study. Data for ED volume, TPA, LOS, and LWBS rates was obtained from Eclipsys Manager Access Program for the 3 years preceding and following RAZ implementation $(2005 / 6 / 7$ versus 2008/9/10) for all CTAS 2 to 5 patients. Outcomes were LOS, TPA, LWBS rates and the number of unplanned revisits within 72 hours. Because the data were highly skewed for TPA and LOS, median and interquartile range (IQR) are analyzed using Mann-Whitney $U$ test. Relative risk (RR) and 95\% confidence intervals (CI) are reported for LWBS and 72 hour return rate. Results: There were 178,597 CTAS 2 to 5 patient visits in the 3 years preceding RAZ implementation and 191,713 in the 3 years post (increase of $7.5 \%$ ). Mean age was 49.9 years before and 48.7 years after RAZ implementation. Median TPA was 35.8 minutes pre (IQR 23-47) and 25.1 minutes (IQR 17-37) post RAZ implementation, showing a median difference of 10.7 minutes (Mann-Whitney $\mathrm{P}<0.0001$ ). Median LOS was 178.1 minutes pre RAZ (IQR 73-233 minutes) and 157.8 minutes after (IQR 59-211 minutes) for a median difference of 20.3 minutes (Mann-Whitney P $<0.0001$ ). LWBS rate was $2.76 \%$ pre RAZ and $2.42 \%$ post RAZ (RR of LWBS post $\mathrm{RAZ}=0.86 ; 95 \% \mathrm{CI}=0.83-0.9)$. Unscheduled return and admission to hospital rate within 72 hours was $1.1 \%$ before and $1.1 \%$ after RAZ implementation (95\% CI 0.94-1.06\%). Conclusion: The implementation of a RAZ was associated with a decrease in ED TPA, LOS and LWBS rate, and no change in the 72 hour unscheduled admission to hospital return rate, despite an increase in patient volumes.

Keywords: rapid assessment zone, emergency overcrowding, throughput

\section{MP11}

High fidelity simulation results in improving clinician performance in the management of massive hemorrhage cases

A. Levy, MD, MEd, G. Pettersen, MD, MSc, F. Gauvin, MD, A. Sansregret, MD, MAEd, S. Lesage, MD, N. Robitaille, MD, Sainte-Justine Hospital University Centre, Montréal, QC

Introduction: Massive haemorrhages are rare but serious complications of paediatric trauma and obstetrical cases. In order to optimize the management of massive haemorrhages, a protocol was implemented at our centre. However, recent audits showed that necessary improvements were to be made to many aspects of its application.We aimed to evaluate the application of a massive hemorrhage protocol and the ability to work in interdisciplinary teams using simulation and targeted training. Methods: Prospective observational study held at the simulation lab of a tertiary mother-child health care facility. Participants were nurses, RTs, orderlies, anesthetists, obstetricians, pediatric emergency physicians, pediatric intensivists and hematologists. Pediatric emergency/intensive care and obstetrical/anesthesia teams were submitted to high fidelity simulated pediatric trauma and post-partum massive hemorrhage scenarios respectively (Simbaby (Laerdal) and Noelle (Gaumard)). Each participant was asked to hold their usual role in an interdisciplinary team. Targeted training consisted of a debriefing session and a presentation reviewing the massive hemorrhage protocol as well as teamwork skills. All sessions were videotaped. Documents 
were given during the first session for future references and to prepare for the post session two weeks later. Confidence questionnaires were filled out during both sessions. Four blinded independent trained raters reviewed the videos and assessment was done using a checklist derived from the protocol and the Mayo High Performance Teamwork Scale. Means and standard deviations of scores for performances were calculated for each scenario and compared using an ANOVA test. Descriptive statistics for the confidence questionnaires were compared using a Mann Whitney test. Results: A total of 62 healthcare professionals involved in 8 interdisciplinary teams participated in the study. Following training, scores for the application of the protocol improved by $24 \%(10-39), p=0.002$. Scores for the ability to work in teams improved by $17 \%(6-28), p=0.006$. Confidence levels in the ability to apply the protocol and work in teams improved by $13 \%$ (11-16), p $<0.0001$. Conclusion: Targeted training using simulation improved participant ability to apply the massive haemorrhage protocol, improved interdisciplinary teamwork skills and confidence levels among participants from all disciplines.

Keywords: interdisciplinary team training, simulation, massive haemorrhages

\section{MP12}

Performance of the Ottawa 3DY Scale as a screening tool for impaired mental status in elderly emergency department patients D. Eagles, MD, D. Otal, BSc, S.K. Sinha, MD, G. Wells, PhD, I.G. Stiell, MD, MSc, University of Ottawa, Department of Emergency Medicine, Ottawa, ON

Introduction: Physicians are not adept at recognizing impaired mental status in elderly Emergency Department (ED) patients, a common and serious entity associated with increased morbidity and mortality. The Ottawa 3DY Scale (O3DY) is a 4 question screening tool that has been previously validated in this population. The overall objective was to improve screening for impaired mental status in elderly ED patients. More specifically, our goal was to assess 1) level of implementation of the O3DY Scale; 2) prevalence of impaired mental status; 3) classification performance compared with the Mini Mental State Exam (MMSE); and 4) agreement between physician and nurse scores. Methods: We conducted a prospective cohort study enrolling patients $\geq 75$ years of age presenting to a tertiary care ED. Patients underwent mental status screening with the O3DY Scale by the bedside nurse and physician. Abnormalities resulted in a comprehensive cognitive evaluation. Descriptive statistics were used to assess level of implementation, prevalence of impaired mental status and sensitivity and specificity compared with the MMSE, using a cutoff of $<25$. Kappa coefficient was calculated. Results: 198 patients, $62.9 \%$ female with a mean age of 84.2 , presented during study hours. Overall, $31.3 \%$ of patients had evidence of impaired mental status. Nurses and physicians screened $64.4 \%$ and $53.7 \%$ of patients, respectively. O3DY by nurses had a sensitivity of $90.1 \%$ (CI $57.1-99.5 \%$ ) and specificity of $60 \%$ (32.9-82.5\%). O3DY by physicians had a sensitivity of $72.7 \%$ (39.3$92.7 \%)$ and specificity of 50\% (32.9-82.5\%). The weighted kappa for agreement between nurse and physician O3DY Scale Scores was 0.79 $(0.65,0.94)$. Conclusion: The Ottawa 3DY Scale is a feasible cognitive screening tool for elderly ED patients which has demonstrated good sensitivity and specificity, particularly when implemented by nurses. Increased identification of cognitive impairment in elderly ED patients will lead to improved care and outcomes in this vulnerable patient population.

Keywords: elderly, mental status, screening tools

MP13

The effect of an initial assessment physician on emergency department throughput
K. van Diepen, MD, D.M. Frost, PhD, J. Sherbino, BSc, MD, MEd, C. Langmann, MD, PhD, W. Bhanich Supapol, PhD, I. Preyra, MD McMaster University, Hamilton, ON

Introduction: Emergency Department (ED) overcrowding is a significant international problem. Placing a physician in triage to rapidly assess patients and initiate diagnostic testing is cited as a method of improving ED throughput and reducing overcrowding. The goal of this study was to assess the impact of an initial assessment physician (IAP) on measures of ED throughput by time of day, patient acuity and admission and consultation status. Methods: This was a retrospective pre-post intervention study that examined the impact of an IAP on measures of ED overcrowding in one academic teaching hospital. Pre and post IAP time periods were matched by time of year (April 2nd to June 2nd, 2012 and April 2nd to June 2nd, 2013). Patients were also grouped and compared by acuity and time of day based on IAP hours (11:00 to 21:00 and 21:00 to 11:00). Data was retrieved from an electronic ED tracking board. The primary outcome measures included time to physician initial assessment (PIA), emergency department length of stay (EDLOS) for non-admitted patients with and without consultation, and EDLOS for admitted patients. The percentage of patients who left without being seen (LWBS) by a physician was a secondary outcome measure. Results: In total, 18371 patient were included in the 4 month study period. Despite an increase in ED volume and admission rates in 2013, the median reduction in time to PIA for all patients seen by the IAP was 86 minutes (110 to 24 minutes; $\mathrm{p}<0.001)$. The median reduction in EDLOS for all non-admitted patients who did not receive a consultation was 27 minutes (211 to 184 minutes; $\mathrm{p}<0.001)$ while the EDLOS for non-admitted patients who received a consultation was reduced by a median of 60 minutes (388 to 328 minutes; $\mathrm{p}<0.001)$. The IAP did not reduce EDLOS for admitted patients. Though low at baseline (1.2\%), LWBS rates decreased by $50 \%$ during IAP hours. Conclusion: Results of this study suggest that an IAP improves ED throughput and measures of ED overcrowding. Keywords: triage physician, emergency overcrowding, throughput

\section{MP14}

General surgery consultations in the emergency department: a multicenter analysis of the appropriateness and timing of surgical referrals

E. Lang, MD, F. Sutherland, MD, L. Mack, MD, D. Wang, MSc, J. Andruchow, MD, A. McRae, MD, University of Calgary, Calgary, AB

Introduction: General surgery consultations constitute an essential service to emergency department (ED) patients on a 24/7 basis. With many of these surgical services provided by trainees, and with growing concern over hours of service provided by residents in surgical training programs, increased attention has been directed to the timing and appropriateness of emergency physician (EP) consultations for surgical evaluation. Methods: We conducted a 6-month multicenter descriptive analysis of General Surgery consultations in four academic emergency departments. Calgary provides a regional model of emergency care with a single general surgery residency training program providing care at all sites which see in excess of 230000 visits annually. We used administrative data including requests for imaging and information on admission and timing of consultation to conduct the analysis. Our primary outcome was the percentage and range of emergency consultation requests resulting in hospitalization. Secondary analyses addressed the percentage and range of consults requested overnight as well as those requested prior to both imaging requests and reported results of CT or US. Results: There were 4167 requests for surgical consultation requested by the EDs during this 6-month period. The overall admission rate was $74.0 \%$, ranging from $64.9 \%$ to $81.3 \%$ for a given site for any one month. Of all general surgical consults that were 
requested, $18.4 \%$ occurred between the hours of 2400 and 0600 , ranging from $12.7 \%$ to $28.5 \%$ by site per month and averaging 1.06 consultations per overnight period across all sites. Only 7\% (range 2.6 9.7\%) of consultations occurred prior to CT or US request by the EP and $21 \%$ (range $11.4-39.9 \%$ ) before CT or US results were reported. Conclusion: Most general surgery consultations are justified and welltimed i.e. resulting in admission and requested after the results of imaging are known. Furthermore, the overnight ED consultation burden does not appear excessive.

Keywords: surgical emergencies, consultation, appropriateness

\section{MP15}

Availability of needed services for older ED patients after discharge following minor injury: results from 1436 patients in the Canadian Emergency Trauma Initiative (CETI) observational cohort study

J.S. Lee, MD, MSc, M. Sirois, PhD, X. Neveu, MSc, J.J. Perry, MD, MSc, A. Worster, MD, R. Daoust, MD, E. Lang, MD, M. Emond, MD, MSc, University of Toronto, Toronto, ON

Introduction: Older ED patients discharged following seemingly minor injuries are at higher risk for loss of function. The availability of needed supportive services (e.g., physiotherapy, nursing, home support) after discharge from the ED is poorly described. Methods: OBJECTIVES: To describe the availability of needed services among independent seniors following discharge from the ED after a minor injury, and whether this differed between sites. STUDY DESIGN: Prospective observational cohort study at 7 Canadian teaching EDs. PARTICIPANTS: Eligible patients were aged $\geq 65$ years, independent in 7 basic activities of daily living, assessed in an ED for a minor injury and discharged home within 48 hours. DATA COLLECTION: We assessed demographics, comorbidities, injury type, cognition using the previously validated Montreal Cognitive Assessment scale (MOCA), Identifying Seniors at Risk (ISAR) scale, and frailty index on eligible consenting patients at baseline. We then conducted followup telephone interviews at 3 and 6 months to assess if patients need supportive services following their injury, including physio, nursing, medical, home modifications, and whether patients had access to such services. We used generalized linear modelling to assess whether site was an independent risk factor predicting lack of access to needed services. Results: We recruited 1436 participants with a mean age of 77.1 \pm 7.5 years $-33.5 \%$ were male. There was significant variability between sites in availability of services. The average unadjusted rate of patients needing services that they could not access was $32.7 \%(95 \%$ CI $29.4 \%$ to $35.9 \%$ ), but this rate varied from a low of $8.1 \%$ to as high as $62.3 \%$. Site was independently associated with an increased rate of needed services that were not accessible, adjusting for covariates listed above. Conclusion: There was significant variability between sites in access to needed services after ED discharge. Future study should look at reasons for variability in access to needed services, and the impact of this wide variability on patient outcomes.

Keywords: geriatrics, emergency department, access to care

\section{MP16}

Increasing the number of junior residents during shifts worsens emergency department crowding: a cross-sectional, single-center study

T. Kawano, MD, H. Hayashi, T. Kimura, University of Fukui Hospital, Fukui Prefecture, Japan

Introduction: Emergency Department (ED) crowding leads to longer patient stays and delays in treatment. Although increasing staff numbers during shifts when crowding is severe can help fit patient demand, it remains unclear how different types of added staff, particularly junior residents, may affect crowding. Here, we sought to determine how addition of junior residents affects ED crowding. Methods: To compare effects of increased staff on ED crowding, we conducted a cross-sectional, single-center study in the ED of a large, teaching hospital in Japan between January and December 2012. Patients who visited the ED during the study period were enrolled. We excluded (1) patients scheduled for visit to $\mathrm{ED}$, and (2) neonates transferred from other hospitals. Types of staff analyzed were nurses, junior residents (defined as the first and second year of residents), senior residents (the third to fifth year of residents), and attending physicians (board-certified). A generalized linear model was performed on length of ED stay for all patients as well as admitted and discharged patients to quantify the effect of the additional staff. Results: During the study period, 27,970 patients were enrolled. Addition of one attending physician or senior resident in shift decreased the length of ED stay for total patients by 3.88 or 1.64 minutes, respectively (IQR, 2.20-5.56 and 0.81-2.48 minutes). Surprisingly, however, one additional junior resident prolonged the length of ED stay for total patients by 0.97 minutes (IQR 0.37-1.57 minutes). An additional nurse in shift had no statistical effect on length of ED stay. In subgroup analysis, one additional junior resident prolonged the length of ED stay for discharged patients by 1.01 minutes (IQR 0.45-1.59 minutes); however, the additional resident had no statistical effect for admitted patients. Conclusion: Our study found that, while adding senior residents and attendant physicians can shorten patient stay in the ED, adding more junior residents might worsen ED crowding.

Keywords: emergency overcrowding, overcrowding, crowding

MP17

Evaluating the turn-around-time of STAT laboratory tests in the emergency department using different test tubes

S. Compeau, MD, S. Matchett, MD, J. Fraser, BN, A. Yeoman, MLT, J. Shea, MD, P.R. Atkinson, MD, M. Howlett, MD, Department of Family Medicine Residency Program, Dalhousie University, Halifax, NS

Introduction: Laboratory (lab) turn-around-times (TAT) are considered an important factor of waiting times in the Emergency Department (ED). Our objective was to determine whether the implementation of Plasma Separating Tubes (PST) (omits the 30 minute clotting step in processing) would change the TAT and rate of specimen hemolysis when compared to the standard Serum Separating Tubes (SST) used in our lab. Methods: We conducted a prospective beforeand-after study with a wash out period. We collected TAT data from five commonly ordered ED lab tests (creatinine, urea, electrolytes, troponin, and NT proBNP) for all ED patients tested during the study period. The two-week control period used standard SST tubes. A oneweek wash out period with PST tubes was followed by two-weeks using PST tubes. Specimen hemolysis rates were collected. Results were excluded if the TAT was 4 minutes or less (data entry error). Analysis used descriptive and two-tailed t-tests statistics (Excel). Results: We included a total of 5610 lab tests, 2819 in the SST group and 2791 in the PST group. Results for in-lab TAT for SST versus PST were analyzed using averages as well as $90 \%$ fractile response time. The average TAT for the creatinine, urea, and electrolyte tests were 43.1 and 40 minutes ( $\mathrm{min}$ ) (CI 27.4-74.6 vs. 20.3-59.7min, $\mathrm{p}=1.0$ ), and the $90 \%$ iles were 64 vs. $60 \mathrm{~min}$ for SST and PST respectively. Similarly for troponin, in comparing the SST and PST groups, the TATs were 55.3 and 52.9min (CI 38.5-72.1 vs. 28.9-76.9min, $\mathrm{p}=0.92$ ), the $90 \%$ iles were 79 vs. $83 \mathrm{~min}$. For NT proBNP, TAT were 56.3 vs. 57.7min (CI 38.9-73.7 vs. 37.5-77.9min, $\mathrm{p}=0.49$ ) and $90 \%$ ile 80 and $81 \mathrm{~min}$ for SST and PST groups, respectively. The hemolysis rates between groups were comparable at $1.1 \%$ in the SST group $(n=32)$ and 
$1.3 \%(n=35)$ in the PST group. Conclusion: Changing the type of test tube used for STAT blood work in our ED did not statistically nor clinically improve the average lab TAT. The $90 \%$ fractile response times for in-lab TAT were significantly longer than our goal of 45 minutes. This demonstrates that other systems issues contribute greater to the TATs than the type of tube used. There was a wide variation in the TAT for both groups, including some low TATs, suggesting that shorter TATs could be achieved if other system issues are addressed.

Keywords: laboratory testing, quality improvement, emergency medicine

\section{MP18}

Why are fast physicians fast?

G. Innes, MD, A. McRae, MD, D. Wang, MSc, E. Lang, MD, University of Calgary, Calgary, $\mathrm{AB}$

Introduction: Fast physicians may achieve high patient volumes using various strategies. Our objective was to determine, in a large city-wide group of emergency physicians (EP), whether physicians achieve high productivity levels (patients per hour) by seeing lower acuity patients, focusing on intake rather than discharge (i.e. delaying discharge decisions and prolonging LOS), or by using admission referrals and complex imaging as dispositional strategies. Our hypotheses were that high volume practice might be associated with lower patient acuity, prolonged LOS for discharged patients, higher complex imaging rates, higher admission rates, and higher bounce-back rates. Methods: All EPs in Calgary belong to an integrated city-wide department that provides all regional emergency care at 3 sites. We prospectively capture MD performance parameters, including patients per-hour, admission rate within CTAS 3, MD throughput time (ED LOS from sign-up to patient discharge) and complex imaging rates. These data were electronically collated for the 141 ED physicians who treated more than 300 patients in 2012. Physicians were stratified by productivity (patients-per-hour) quartile. Productivity quartile was associated with key outcomes using Pearson's or Spearman's r, as appropriate. ANOVA, with Tukey's procedure, were used to determine statistical significance of differences between multiple groups. Results: There was no correlation between physician productivity and mean CTAS acuity (Pearson's $r=0.02$; $\mathrm{p}=0.80$ ). There was weak negative correlation between EP productivity and LOS $(\mathrm{r}=-0.35 ; \mathrm{p}<0.01)$ and admission rates $(\mathrm{r}=-0.35 ; \mathrm{p}<0.001)$. There was weak negative correlation between productivity and complex imaging rates $(r=-0.30 ; p<0.001)$. ANOVA showed that the most productive physicians had the lowest CTAS 3 admission rates (mean admission rate for $\mathrm{Q} 1=18.2 \%$; $\mathrm{Q} 2=16.8 \%$; $\mathrm{Q} 3=16.2 \%$; $4=15.4 \%$; $<<0.001)$. High productivity physicians also had the shortest CTAS 3 throughput times (mean LOS for Q1=2.1hr; Q2=1.7hr; Q3=1.7hr; Q4=1.5hr; $\mathrm{p}<0.001$ ). Similar relationships were seen for complex imaging $(\mathrm{p}=0.009)$. There was no relationship between MD productivity and patient bounce back rates $(r=0.10 ; p=0.26)$. Conclusion: In this setting, high volume physicians had the same acuity mix and tended to have shorter lengths of stay, lower admission and imaging rates, and similar bounce back rates. Physicians did not achieve higher productivity by seeing lower acuity patients, referring more patients for admission, delaying discharge decisions, or ordering more imaging tests.

Keywords: emergency services, efficiency, access

MP19

The effect of ketamine on intracranial and cerebral perfusion pressure and health outcomes: a systematic review of the literature L. Cohen, MDCM, V. Athaide, MD, N. Rose, MD, C.M. Hohl, MDCM, MHSc, UBC Department of Emergency Medicine, Vancouver, $\mathrm{BC}$
Introduction: Emergency departments see a high volume of undifferentiated critically ill patients who require airway management before traumatic or other neurological injuries have been ruled out. Emergency physicians' reluctance to use ketamine in undifferentiated critically ill patients is largely based on case reports published over forty years ago. Our objective was to synthesize the available evidence on the effect of ketamine on intracranial pressure, cerebral perfusion pressure, neurologic outcomes, intensive care unit length of stay and mortality compared to other intravenous sedatives. Methods: We developed a systematic search strategy and applied it to the MEDLINE and EMBASE electronic reference databases. Two study authors independently performed title, abstract, and full text reviews, and abstracted data from all included studies using standardized forms. Data from randomized controlled trials and controlled clinical trials were synthesized in a qualitative manner, as the study populations, designs and reported outcomes were heterogeneous. Results: We retrieved 4299 titles, of which 8 studies met our inclusion criteria. Of these 8 studies, we found 3 RCTs and 3 controlled clinical trials examining the effect of ketamine on intracranial and cerebral perfusion pressure that demonstrated no significant difference in these outcomes when compared to other sedative or induction agents. Four RCTs examined the effect of ketamine on neurologic outcome, none of which demonstrated significant differences between groups. Two RCTs examined ICU length of stay, and found no difference between patient groups. Only one RCT examined mortality, and found no difference between patient groups. Conclusion: Based on the available peer-reviewed literature, ketamine does not appear to adversely impact intracranial and cerebral perfusion pressure, neurological outcomes, ICU length of stay, or mortality compared to other sedative agents. However, none of the studies were of high-quality, and none was adequately powered to detect clinically significant differences in patient-oriented outcomes. Larger well-designed RCTs are required to definitively answer these questions.

Keywords: ketamine, intracranial pressure, rapid sequence intubation

\section{MP20}

Smoking-related medical conditions in the emergency department: prevalence and accuracy of patient perception that their visit may be related to smoking

A. Tsai, J. Tsai, BSc(Pharm), L. Stewart, MPH, K. Cheung, MD, MPH University of British Columbia, Vancouver, BC

Introduction: Worldwide, smoking is the single leading cause of preventable deaths and disability. In 2004, the US General Surgeon's Report provided a list of medical conditions that were causally found to be smoking-related. In this study, we determine the prevalence of smoking-related conditions as the Emergency Department (ED) discharge diagnosis among tobacco users who visited a tertiary care academic ED. We also sought to determine which medical conditions were more or less likely to be accurately perceived by patients as being smoking-related. Methods: All adults $\geq 18$ years of age who had used tobacco within the last 30 days prior to their ED visit and who were enrolled in a randomized controlled trial (ClinicalTrials.gov, NCT01454375) from Dec.1, 2011 to Aug.31, 2012 were included in the study. As part of the randomized controlled trial, all patients were asked whether or not they perceived that their ED visit could be due to a smoking-related condition. Blinded abstractors coded ED diagnoses from patient charts as smoking-related or not smoking-related. All smoking-related conditions were placed under 7 major disease categories: cardiovascular disease, neoplasm, respiratory disease, reproductive complication, peptic ulcer disease, post-op complication, or dental disease. Results: Eight hundred and twelve patients were included; the mean age was 40 years and $61 \%$ were male. In total, 
$14 \%$ had a smoking-related condition - $56 \%$ were respiratory diseases, $16 \%$ cardiovascular disease, $7 \%$ peptic ulcer disease, $7 \%$ post-operative complications, $7 \%$ dental disease, $5 \%$ neoplasm, and $2 \%$ reproductive complications. Of the conditions identified, $33 \%$ were correctly perceived by patients to be smoking-related. Cardiovascular diseases, neoplasms, and respiratory diseases were more likely to be accurately perceived by patients to be smoking-related. Dental diseases, peptic ulcer disease, post-operative complications, and reproductive complications were less likely to be perceived as smokingrelated. Conclusion: In this study, $14 \%$ of all visits to the ED among smokers were secondary to a smoking-related condition. Only $33 \%$ of smoking-related conditions were accurately perceived by patients to be related to tobacco. Further patient education is required to increase awareness of smoking-related conditions, which may ultimately lead to increased quit rates

Keywords: smoking cessation, patient perception, health education

\section{MP21}

Derivation of predicting tools of functional decline in independent seniors with minor injuries in the ED

M. Emond, MD, MSc, M. Sirois, PhD, J.S. Lee, MD, MSc, J.J. Perry, MD, MSc, E. Lang, MD, A. Worster, MD, R. Daoust, MD, N. Le Sage, MD, MSc, Université Laval, Québec, QC

Introduction: Nearly $15 \%$ of older patients discharged from the ED following seemingly minor injuries are at higher risk for loss of function. A clinical decision tool is needed to predict functional decline. OBJECTIVES: We aim to derive clinical decision rules to ascertain functional decline at 3 months in community-dwelling seniors. Methods: A prospective longitudinal study was conducted in 7 Canadian university-affiliated EDs between April 2009 and. April 2013. Eligible patients were aged $\geq 65$ years, independent in 7 basic activities of daily living were assessed in an ED for a minor traumatic injury and discharged back home within 48 hours of presentation. Eligible consenting patients had functional, cognitive assessments and frailty index completed either at initial ED visit or shortly after. We conducted inperson and telephone follow-up interviews at 3 months to assess subsequent functional status using the Older American Resource and Service Scale (OARS). Main outcome: A drop of 2 points on the OARS scale was considered a positive decline on follow-up. Univariate and recursive partitioning analyses were used to derive clinical decision rules. Results: We recruited and followed 1277 Canadian independent seniors with a mean age of $77.1 \pm 7.5$ years. 162 seniors $(12.7 \%)$ had a positive outcome. Three clinical decision tools were derived using a combination of 1) age, 2) occasional use of a walking aid, 3) SOF frailty index, 4) previous falls history, 5) Number of outings per week, 6) comorbidities and 7) Timed-up-go score. Sensitivity values range from 0.88 to 0.97 , specificity from 0.40 to 0.47 and a good negative predictive values from 0.96 to 0.99 . The best prediction tool failed to identify 6 senior decliners at 3 months. Conclusion: Clinical decision tools were derived to predict functional decline in previously independent seniors with minor injuries in the ED. Future studies are needed to validate and refine clinical decision tools at 3 months following discharge from ED and later at 6 months

Keywords: functional decline, seniors, minor injuries

\section{MP22}

External validation of the criteria defining « clinically important» brain injury in patients with mild TBI

J. Lessard, MD, R. Daoust, MD, J. Paquet, PhD, J. Giguère, MD, D. Gilbert, MD, M. Roy, MD, M. Vincent, MD, J. Chauny, MD, MSc, Hôpital Sacré-Coeur de Montréal, Department of Emergency Medicine, Montréal, QC
Introduction: The Canadian C-Spine/CT-Head Study was designed to determine the need for imaging in head and neck trauma. It introduced the concept of « important brain injury » (IBI), used to anticipate need for surgical intervention and admission. The objective of this study is to externally validate these criteria in a trauma center. Methods: This is a post-hoc study of a prospectively collected cohort. Mild traumatic brain injury (MTBI) was diagnosed by a neurosurgeon and the head CT was read by a neuroradiologist. Need for surgical intervention or admission was at the discretion of the treating physician. Follow-up was organized by a clinician nurse at the hospital or by telephone. All brain injuries on CT were considered IBI unless the patient was neurologically intact and had one of the following lesions : (1) solitary contusion $<5 \mathrm{~mm}$ in diameter, (2) localized subarachnoid blood $<1 \mathrm{~mm}$ thick, (3) subdural hematoma $<4 \mathrm{~mm}$ thick, (4) isolated pneumocephaly, or (5) closed depressed skull fracture not through the inner table, as proposed by Stiell and al. Sensitivity and specificity of IBI were measured with $95 \%$ confidence interval. Results: 803 patients were recruted between 2008-2012, 604 (75.2\%) admitted and 199 $(24.8 \%)$ discharged from the ER. 38 patients required neurosurgical intervention and all had characteristics of IBI on head CT, for a sensitivity of $100 \%$ (CI 90.7-100), and a low specificity of $28.5 \%$ (CI $25.3-$ 31.8 ). As for the need of admission, the IBI criteria had a sensitivity and specificity of $80.1 \%$ (CI 76.9-83.3) and 50.8\% (CI 43.9-57.7). The correlation between the need for admission and the presence of IBI was very strong (Chi-2 $65.3 \mathrm{p}=0.001)$. Conclusion: The preliminary results of the study externally validate the concept of IBI for minor head injury patients, regarding the need for neurosurgical intervention, at the cost of a low specificity. We will next determine other factors which could have influenced the need for hospital admission.

Keywords: mild traumatic brain injury, clinical decision rule

\section{MP23}

Predictors of adverse events associated with sedation choice in electrical cardioversion

D. Clinkard, MSc, I.G. Stiell, MD, MSc, C.M. Clement, R.J. Brison, MD, B.H. Rowe, MD, MSc, P. Dorian, MDCM, MSc, B. Borgundvaag, $\mathrm{PhD}, \mathrm{MD}$, D. Redfearn, MD, T. Langhan, MD, E. Lang, MD, K. Magee, MD, S. Rose, MD, R. Stenstrom, MD, PhD, D. Eagles, MD, G. Wyse, MD, PhD, J.J. Perry, MD, MSc, D. Birnie, MD, G. Wells, PhD, A. McRae, MD, Queen's University, Kingston, ON

Introduction: Electrical cardioversion (EC) is a commonly used procedure for symptomatic atrial fibrillation and flutter. There is little evidence as to the comparative safety of various sedation medication options for EC. Methods: A secondary analysis of two prospective cohort studies of patients with symptomatic AF in eight Canadian EDs from 2008 to 2012. Associations between sedation medication choices and adverse events (hypotension, hypoxia, bradycardia, tachycardia, apnea, aspiration or oral airway required) were examined using multivariable logistic regression analysis. Sedation choices that were examined included propofol alone, propofol \& midazolam, propofol \& fentanyl, propofol \& midazolam \& fentanyl, and propofol \& other (rare sedation choices). Potential confounders included in the model included oral antihypertensives, use of IV rate control medication prior to EC, age $>65$ and initial SBP $<120$. Results: Of the 999 patients who underwent EC, 982 had sedation procedure documented. Mean age was 59.8 (range 18-97), 67.9\% were male, average HR 121 (50 to 237 ), and SBP of 130 (174 to 234). There were 78 adverse events (7.9\%). The most commonly used sedation regimen was the combination of fentanyl and propofol, used in $565(57.5 \%)$ of sedations. Propofol alone was used in 372 sedations (33.2\%)., with propofol, fentanyl and midazolam used for 18 sedations $(1.8 \%)$ and propofol and midazolam used in $16(1.6 \%)$. Multiple other combinations made up the 
remaining 56 sedations $(5.7 \%)$ of sedation choices. Compared to the propofol alone, after adjusting for potential cofounders, only the combination of propofol, fentanyl and midazolam was found to be significantly associated with adverse events compared to propofol alone (OR $4.29, \mathrm{p}=0.03$ ). Conclusion: We have shown that the combination of propofol, an opioid and a benzodiazepine for cardioversion sedation is associated with significantly more adverse events then propofol alone. We did not identify an increased risk of adverse events from the addition of fentanyl to propofol for sedation for EC.

Keywords: cardioversion, sedation

\section{MP24}

Emergency physician attitudes toward CT ordering

J. Worrall, MD, A. Kadhim-Saleh, BHSc, MSc, M. Gatien, MD, M. Taljaard, PhD, J.J. Perry, MD, MSc, University of Ottawa, Department of Emergency Medicine, Ottawa, ON

Introduction: Despite increased awareness of the risks of radiation, emergency physicians (EPs) have increased use of CT scanning in the last decade. Our objectives were to determine 1) which factors influence EPs self-reported decision to order CT scans; and 2) their views on their colleagues' ordering practices. Methods: A paper survey was distributed to all attending EPs in the two emergency departments of an urban tertiary care university hospital, with more than 140,000 visits annually. The survey instrument was pilot tested on senior emergency residents for content and comprehensibility. Reminder emails, to encourage completion, were sent at two weeks and two months. The survey included 5 demographic questions, a list of 10 factors which may influence CT ordering (ranked on a 4 point Likert scale), and 5 questions on beliefs about CT ordering (on a 5 point Likert scale). Anonymized data were recorded in a database and analyzed using descriptive statistics. Results: The response rate was 76\% (51/67 EPs surveyed). Respondents were $66 \%$ male and $59 \%$ were over 40 years old. Respondents completed residency training at our centre $(45 \%)$, in another national program (45\%), or outside the country $(10 \%)$. Most EPs believe CTs increase lifetime risk of cancer (90.2\%), and that physicians order more CTs than they used to $(96.1 \%)$. A majority of EPs $(62.7 \%)$ believe they order about the same number of CTs as their colleagues, $29.4 \%$ believe they order fewer, while $7.8 \%$ believe they order more. Only $17.6 \%$ of EPs believe they should order fewer CTs, but $33.3 \%$ believe their colleagues should order fewer. Physicians reported the following factors "moderately" or "heavily" influence their decision to order CTs: risk of missed diagnosis ( $92.2 \%$ of EPs), risk of radiation $(88.2 \%)$, lifetime number of CTs a patient has had (88.2\%), applicable clinical decision instrument (74.5\%), consultant wishes $(52.9 \%)$, risk of contrast $(49.0 \%)$, ED flow $(43.1 \%)$, medicolegal risk $(42.0 \%)$, what colleagues would do (33.3\%), and patient wishes (7.8\%). Conclusion: While most physicians acknowledge that CTs may increase risk of cancer and that EPs order more CTs than in the past, few acknowledge that they should order fewer CTs. Similarly, only few self-identify as high-orderers. The risk of a missed diagnosis is the factor most reported to influence ordering decisions.

Keywords: imaging, utilization, clinical decision making

\section{MP25}

Reliability of Balance Error Scoring System (BESS) test for concussion in different field conditions

A. Azad, MD, S. Al Juma, MD, J. Delaney, MDCM, J.A. Bhatti, PhD, McGill University Health Centre - Department of Emergency Medicine and Sport Medicine, Montréal, QC

Introduction: Concussion is common in contact sports. Undiagnosed concussion, in the worst case scenario, can lead to a permanent disabil- ity. Balance Error Scoring System (BESS) is a simple and effective tool for screening concussion. Like other tests, it requires athletes to be barefooted, usually in closed room environment. In order to assess BESS use with cleats-on in field conditions, this study aimed to compare BESS test performance with cleats-on and barefooted. Methods: The setting of this repeated measure design study was McGill University sport clinic. Participants were from McGill University football and soccer teams. The study included male and female players aged 18- 30 years providing informed consent. Those with a history of musculoskeletal injuries in the past 6 month, vestibular impairment and deemed unfit to play were excluded. Participants were asked to perform modified BESS test with three stances (two legs, single leg and tandem) in three conditions which were barefooted, with cleats on turf, and with cleats on hard surface. Each performance was scored by three observers. Performances were averaged from three observers and paired $t$ tests were used to assess mean differences in BESS performances with cleats-on conditions compared to barefooted condition. Reliability was assessed by intra-class correlation coefficient (or ICC). Results: A total of 60 athletes from football ( $\mathrm{n}=39$, all males) and soccer $(\mathrm{n}=21,11$ males and 10 females) teams. Average age was 21.1 years (standard deviation $[\mathrm{SD}]=2.1)$. Participants on average scored $28.4(\mathrm{SD}=1.5)$ points out of 30 for barefooted condition. Modified BESS scores were on average two points less than barefoot conditions for both cleats-on turf condition (difference $=2.1 ; 95 \%$ confidence interval $[95 \% \mathrm{CI}]=1.6$, 2.1 ) and cleats-on hard surface condition (difference $=1.9 ; 95 \% \mathrm{CI}=1.3$, 2.4). A moderate to high inter-observer reliability $(0.61 \geq \mathrm{ICC} \leq 0.74)$ was observed for BESS test under three conditions. Conclusion: BESS appeared reliable with cleats-on conditions. This study indicates a 2 points lower cut-off than an expected cut-off for suspected concussion when using BESS with cleats-on field conditions.

Keywords: cleats, contact sport, mild brain injury

\section{MP26}

Communication in ED resuscitations: analysis of information sharing among emergency department team members during resuscitation of critically ill patients

G. Mastoras, MD, L.A. Calder, MD, MSc, M. Rahimpour, BA, B. Sohmer, MD, B. Weitzman, MD, A. Cwinn, MD, A. Parush, PhD, The Ottawa Hospital, Ottawa, ON

Introduction: Effective communication and collaboration among team members during Emergency Department (ED) resuscitations is essential for both team efficacy and patient safety. To date, little attention has been given to the existing processes underlying communication in ED resuscitation. This qualitative, observational study sought to develop a better understanding of information-sharing among team members in this high-stakes environment. Methods: A prospective sample of resuscitations were observed in The Ottawa Hospital Civic Campus Emergency Department between December, 2012 and April, 2013. Individual observations were conducted by the primary author and proceeded from ED team activation until patients were deemed stable, deceased, or care was handed off to consulting services. Communications between members of the resuscitation team (e.g., MDs, RNs, RTs) were captured using a structured, piloted data collection form based on concepts from health-care communication research. Data analysis was carried out in an iterative fashion, using Communication Analysis to identify common thematic elements and develop a conceptual framework. Data were collected until saturation was achieved. Results: A total of five hours of ED resuscitations ( $n=12)$ were observed and recorded, representing a breadth of critical presentations. Key domains of communication were identified and categorized by content, with Therapeutic Actions, Clinical Findings, and Resuscitation Status comprising over half of all communications. The 
subsequent analysis aimed to group these communication elements according to context and targeted recipients, which resulted in the identification of key information-sharing categories of relevance to ED resuscitation. Conclusion: This study is among the first to analyze information sharing among ED teams in situ during resuscitations. While limited to a single centre, the findings contribute to a more comprehensive model of ED team communication. These findings are relevant to our understanding of team cognition and work processes in ED resuscitation, and have implications for the design of augmentative systems to support team situational awareness.

Keywords: teamwork, information sharing, emergency department resuscitation

MP27

Evaluation of a clinical score for skull radiography of young children with isolated head trauma

J. Gravel, MD, MSc, S. Gouin, MDCM, D. Chalut, MD, L. Crevier, MD, B. Masse, PhD, Université de Montreal, Montréal, QC

Introduction: Background: A clinical score was reported in 2010 by Bin et al. to identify head-injured infants that are at higher risk of skull fracture. Objective: To determine the criterion validity of the clinical score to identify skull fracture among children younger than 2 years old with head trauma and no need for head tomography. Methods: Method: A prospective cohort study was conducted in two pediatric emergency departments. Participants were all children younger than 24 months who sustained a head trauma and for whom head tomography was not highly recommended according to the PECARN head CT scan rule. The exposure of interest was the clinical score (from 0 to 8 ) composed of the age of the patient, the size and location of the hematoma. The previous study suggested that a score higher than two would be predictive of skull fracture. The primary outcome was the presence of a skull fracture according to radiological report. All participants were initially evaluated by a physician using a standardized datasheet before radiological evaluation. Skull radiography ordering was left at the primary physician's discretion. The primary analysis was the association between the clinical score and presence of a skull fracture. It was estimated that a sample of 50 cases of fracture would provide a width of + or -0.05 if the sensitivity of the score was higher than 0.90 . Results: Results: A total of 765 patients were recruited during the study period. Among them, 271 had a radiological evaluation and 50 had a skull fracture. Most children had low clinical score but 209 had a score higher than 2 . A clinical score above 2 points resulted in a sensitivity of 0.86 (95\%CI: $0.74-0.93)$ and a specificity 0.77 (95\%CI: $0.74-0.80)$. Conclusion: Conclusion: The clinical decision score demonstrated a moderated sensitivity to identify children at risk of skull fracture. Further studies are required to derive a useful clinical decision rule for young children with head trauma.

Keywords: pediatric, trauma, minor head injury

\section{MP28}

Emergency department transient ischemic attack novel clinical pathway: etiology-based triage to improve patient quality of care

J. Cheung, K. Treasurywala, BSc, MD, W. Fung,, J. Kolesnik, A. Wang, D. Sahlas, MD, D. Lin, MD, McMaster University, Hamilton, ON

Introduction: Recent evidence suggests that clinical decision tools such as the ABCD2 score are inaccurate in identifying patients at highrisk for stroke following clinical presentation of a transient ischemic attack (TIA). The risk of stroke within 7 days following TIA is high, and secondary prevention is important to decrease morbidity and mortality, including carotid endarterectomy for symptomatic carotid artery stenosis (CAS) and/or initiation of antiplatelet therapy. The goal of this study was to implement a clinical pathway in the Emergency Depart- ment (ED) based on TIA-etiology to improve patient access to diagnostic imaging, outpatient follow-up, and potential surgical intervention. Methods: A novel clinical pathway was devised and implemented in the ED based on potential etiologic mechanisms of TIA to facilitate investigation of symptomatic moderate to high-grade CAS and subsequent triage to the regional Stroke Prevention Clinic (SPC) for outpatient follow-up. Patients above the age of 18 years old diagnosed with TIA in the ED of two large academic centers and who were referred to the SPC from November 2012 to February 2013 were prospectively identified and their clinical outcomes followed for one month. A historical cohort of patients diagnosed with TIA in the ED prior to the implementation of the clinical pathway was reviewed from January to December 2011 as a comparison group. Primary data extraction included days to carotid artery ultrasound, and days to SPC follow-up. Secondary data extraction included percentage of patients receiving EKG, neuroimaging, and/or initiation of antiplatelet therapy. Results: Prior to the implementation of the clinical pathway, the median time to carotid artery ultrasound for ED patients referred to the SPC in whom it was ordered (49/94) was 6 days. With the clinical pathway, the median time to carotid artery ultrasound significantly decreased to 1 day $(\mathrm{p}<0.001)$, despite more carotid artery ultrasounds being ordered (77/81). A significantly higher proportion of patients received brain imaging (CT Head) with the implementation of the clinical pathway, increasing from $86.2 \%$ pre-clinical pathway to $96.3 \%$ post-clinical pathway $(\mathrm{p}<0.05)$. In patients without a diagnosis of atrial fibrillation, $96.1 \%$ were discharged with an antiplatelet agent compared to $46.2 \%$ prior to the clinical pathway ( $<<0.001)$. There was no change in percentage of patients receiving EKGs pre or post-implementation of the clinical pathway. Conclusion: The clinical pathway based on potential vascular etiology implemented during this study for patients diagnosed with TIA in the ED utilizes rapid outpatient access to carotid artery ultrasound in order to facilitate appropriate triage for SPC follow-up. The pathway significantly improved the median time to receive carotid artery ultrasound, as well as the proportion of patients receiving brain imaging, and antiplatelet therapy. Further research is required to investigate the effect on patient morbidity and mortality.

Keywords: transient ischemic attack, stroke prevention clinic, carotid artery ultrasound

\section{MP29}

Do you feel safe at work? A survey of residents' perception of safety in a New York City emergency department

M. Rubin, MD, Metropolitan Hospital Center, New York Medical College, NY

Introduction: A shooting in a New York City hospital prompted residents to question; "how safe are we at work?" Residents in one of New York City's oldest emergency residency programs are aware that they work in city hospitals that serve a low socioeconomic status demographic and that many of their patients are under the influence of drugs, alcohol and have a previous history of violence, psychiatric illnesses, belong to gangs and have access to firearms. Most residents have come to regard the workplace violence that they must endure as simply "part of the job." The objective of this study is to gauge the residents' perspective on workplace violence in one intercity residency program and to develop recommendations that can be used to further improve safety in the studied ED. Methods: All twenty-four emergency residents in the program were sent an electronic survey to be completed at their leisure. The survey consisted of 37 multiple-choice questions and 6 essay questions. The survey focused on residents' feeling of safety and personal accounts of physical assaults that occurred in the ED. Survey questions were divided into three categories; 1) security measures, 2) violent experiences and 3) root cause analysis essay response questions. Results: Twenty-three of the 24 emergency 
residents responded to the survey. One hundred percent of residents who responded stated they had been verbally abused by patients. Many reported verbal abuse as a daily occurrence. Seventy-eight percent of residents stated they have been the victims of physical assault by patients while working in the ED. Thirty-nine percent of residents have had to confiscate potential weapons from emotionally disturbed patients. Conclusion: This study demonstrates that residents in the ED under study are coping with nearly three times the incidence of violent acts when compared to the United States national average. The study contains multiple recommendations to improve the safety in the ED stemming from the residents feedback, a review of state and federal laws as well as a comprehensive literary search.

Keywords: safety, emergency resident, New York City

\section{MP30}

Prospective characterization of follow-up imaging/culture results after patient disposition in the emergency department

E.S. Kwok, MD, S.V. Vickerman, BSc, University of Ottawa, Ottawa, ON

Introduction: Radiological and microbiological investigations are often performed in the Emergency Department (ED) with the final results returning at a later time after patients are physically out of the ED. There is little published evidence characterizing the incidence, nature, and potential clinical and administrative impact of such delayed results. We sought to evaluate our own quality assurance (QA) process with respect to followup imaging and microbiology reports. Methods: This prospective study took place at a tertiary care teaching ED campus over a 2 month period. A dedicated QA nurse reviewed all final radiology and microbiology reports during this time, and all results that required additional actions (such as simply informing the patient, forwarding results to a Family Physician, prescribing new antibiotics, or asking the patient to return to the ED) were included in the study. Any ambiguity with regards to the type of action needed were reviewed with an emergency physician on duty. A structured data collection sheet was used to capture relevant details. Primary outcomes include 30-day mortality, hospital admission, and immediate return visit to ED for reassessment. Data were entered and analyzed in an excel database and presented using descriptive statistics. Results: A total of 226 cases of delayed final reports were identified by the QA nurse requiring further actions. 118 were related to imaging reports and 108 were related to microbiologi$\mathrm{cal} /$ culture results. The average time from patient's initial visit to final report identification by the QA nurse was 1.94 days and 3.46 days, respectively. There were no 30 -day mortalities. The top 3 most frequent imaging categories were:1)plain-film chest radiography(29.7\%), 2)computed tomography of the abdomen(18.6\%), 3)plain-film musculoskeletal radiography(17.8\%), with $11.0 \%$ of these cases requiring new prescriptions to be filled, $7.6 \%$ requiring immediate return visit to the $\mathrm{ED}$, and $0.8 \%$ being admitted. The top 3 most frequent culture categories were: 1)urine(47.2\%), 2)blood(13.9\%), 3)skin and soft tissue(10.2\%), with $56.5 \%$ of these cases requiring prescriptions to be filled, $7.4 \%$ requiring immediate return visit to the $\mathrm{ED}$, and $0.9 \%$ being admitted. Conclusion: The majority of final imaging and culture results requiring further action become available days after the initial ED visit. While many were managed on an outpatient basis, followup results placed a tremendous strain on QA resources. Additionally a significant proportion required immediate ED reassessment, underscoring the importance of a robust QA process. Further research is needed to examine the appropriateness and utility of ordering these investigations in the ED in the first place, and to guide development of more efficient administrative methods of addressing common QA items.

Keywords: quality assurance, patient safety, administration

\section{MP31}

Helmet wearing practices and barriers to use in Toronto bikeshare (Bixi) users: a control matched study

S.M. Friedman, MD, MPH, M. Adamson, BPHE, P. Cleiman, MD, M. Winters, MSc, PhD, K. Oleksak, BScE(Hons), I.M. Mohabir, BSc, R. Ta, BSc, MSc, K. Reiter, BMSc, University Health Network, Toronto, ON

Introduction: Toronto bike-share (Bixi) users have made approximately $1,300,000$ trips since 2011 . Observed helmet use is $21 \%$. We seek to characterize helmet use patterns and barriers to use among bike-share users, so as to help identify strategies to increase helmet use. Methods: Standardized survey of bike-share users at semi-random distribution of Bixi stations. By maintaining a ratio of one helmet wearer (HW): two non helmet wearers (NHW) on each survey period we controlled for location, day, time, and weather. Results: Surveys were completed on 545 (180 HW, $365 \mathrm{NHW})$ unique users at 48 locations, from Nov 2012-Aug 2013. A higher proportion of females wear helmets (F: $41.1 \%$, M: $30.9 \%, \mathrm{p}=0.0423$ ). NHWs were younger than HW (NHW mean, median age 34.4, 32.0 years vs HW 37.3, 34.5, $\mathrm{p}=0.0018$ ). Groups did not differ by employment status, education, or income. Helmet ownership was lower among NHWs (NHW $62.4 \%$ vs HW: $99.4 \%, \mathrm{p}<0.0001$ ), as was personal bike ownership (NHW 65.8 $\%$, vs HW $78.3 \%, \mathrm{p}=0.0026$ ). Non helmet wearers were less likely to always wear a helmet when on their own bike (NHW: $22.2 \%$ vs HW: $66.7 \%, \mathrm{p}<0.0001$ ), and less likely to wear a helmet always or most of the time when on Bixi (NHW: $5.8 \%$ vs HW: $92.3 \%$, p < 0.0001). Both groups, but more HWs, had planned to use Bixi when leaving their houses (HWs $97.2 . \%$ vs NHW $85.2 \%$, p $<0.0001$ ). The main trip purpose in both groups was to get to work (HW: $88.3 \%$ vs NHW $84.1 \%$, $\mathrm{p}=0.19$ ). Both groups were primarily one year subscribers (NHW 85.2 $\%$ vs. HW $95.6 \%$ ), NHWs were more likely than HWs to cite helmets as inconvenient ( $64.7 \%$ vs $21.7 \%$ ), unnecessary ( $11.2 \%$ vs $1.2 \%$ ), or lack of ownership (19.7\% vs $0.6 \%)$ as their primary barriers to helmet use (all $\mathrm{p}<0.0001)$. Groups did not differ in their views of the safety of cycling in Toronto. NHWs were more likely to report that that they would wear a helmet more $(61.4 \%$ NHW vs. $13.9 \%$, HW p $<0.0001)$, and/or cycle less (22.5\% NHW vs $4.4 \% \mathrm{HW})$ if helmet use was mandatory. Approximately half of NHWs endorsed using loaner helmets ( $28.8 \%$ very likely, $24.7 \%$ likely) if made available at Bixi stations. Conclusion: Non helmet wearers tended to be male, younger, and less likely to use helmets on their personal bikes. Interventions to improve helmet usage should target both personal and bike share users. Legislating helmet use and provision of rental helmets could improve helmet use among bike-share users, but results suggest some risk of reduced cycling with legislation.

Keywords: cycling, helmet, injury

\section{MP32}

Effectiveness of individualized inter-professional care plans for heavy emergency department users

B. Borgundvaag, PhD, MD, J.J. Hunter, MD, C. Hunchak, MD, MPH, K. Van Den Broek, MScN, R.G. Maunder, MD, L. Waisberg, MSW, H.J. Ovens, MD, University of Toronto, Toronto, ON

Introduction: Heavy Emergency Department (ED) users, the few patients who use a disproportionately large amount of ED resources, present a mismatch between medical needs and available system resources. Such patients have a high frequency of mental health and substance abuse diagnoses, and chronic needs, which are not well matched to services available in the ED. Their complexity and visit frequency often leads to frustration for both patients and care providers. Methods: Using an interdisciplinary team, we created individualized care plans for 108 individuals identified as the heaviest users of the ED in the previous year, or identified by staff as likely to benefit from a 
care plan. Our goal was to improve care, reduce visit frequency, and support staff in providing care under challenging situations. Care plans were constructed by reviewing medical records of all known medical, psychological and social problems, and contacting known providers to identify known sources of care and other pertinent issues. Information was recorded and input was sought from relevant medical specialists and community service agency workers. Plans were designed to focus on medical, psychological and social needs and to improve communication among providers and consistency of approach. Visit information was recorded and compared for the 3 quarters pre- and post-care plan implementation. Results: Instituting care plans reduced ED visits among this group ( 8.8 pre to 5.9 post, $\mathrm{p}<.001$ ). In addition, for the $38 \%$ of patients who most clearly improved (11.0 pre to 3.8 post, $\mathrm{p}<.001)$, there was also reduced length of stay $(\mathrm{LOS}) / \mathrm{visit}(289 \mathrm{~min}$ pre to 187 min post, $p=.003$ ). Care plans were extremely well received by providers who felt they were able to deal with these patients' needs more effectively. Conclusion: Heavy ED users highlight a gap in most ED systems, and represent a significant challenge to effective service delivery. Using an interdisciplinary approach, we have been able to develop care plan guidelines for these patients which have demonstrated a significant reduction in ED visits as well as LOS for a large percentage of this population.

Keywords: care plan, heavy emergency department users, emergency department

\section{MP33}

Content analysis of parents' needs and expectations for emergency care of children

T. Principi, MD, M. Lima, S.C. Porter, MD, The Hospital for Sick Children, Toronto, ON

Introduction: Understanding parental needs and expectations is essential to idealized parent-provider communication and patient-centered care. Increasing adoption of electronic medical record systems in emergency departments (EDs) makes exploration of electronic, patient-driven documentation relevant. Our objective was to evaluate the potential value of information provided electronically by parents regarding needs and expectations for emergency care. Methods: This was a planned content analysis of parents' electronic survey data collected during a prospective cohort study of satisfaction of emergency care in an urban, tertiary level pediatric ED. Recruitment was limited to English-speaking parents. The survey was deployed using REDCap on a tablet platform. Parents answered free-text questions regarding worries, questions for providers, causality of symptoms, as well as a multiple choice question on specific requests for care. Potential value of parents' information was defined using Hoppa's standard (PEC 2011) which included the constructs of readability, relevance to final diagnosis, and reasonableness of requests. A single trained reviewer judged the primary outcome, with a second reviewer checking uncertain cases. Results: 725 eligible patients were seen during the study period. $652(90.0 \%)$ electronic surveys were collected from enrolled parents. $522(80.1 \%, 95 \%$ CI $77.0 \%-83.2 \%)$ of surveys had potential value. The total median word count per survey was 27 (IQR 15-45), with a range of 2 to 242 . Of the 652 forms, $467(71.6 \%)$ had at least 1 parental request for care. The median number of requests was 1 (IQR $0-2$ ). Of these, the most frequent request was "medication to treat the problem" $(224 ; 48.0 \%)$, followed by "blood tests" $(175 ; 37.5 \%)$ and "see a specialist" $(127 ; 27.2 \%)$. Conclusion: This study affirms the potential value of parents' perspectives captured using a novel electronic interface. Electronic data capture of relevant data directly from patients holds promise for future innovations in a shared electronic health record.

Keywords: emergency medicine, needs assessment, electronic health records

\section{MP34}

Comparison of emergency department visits for homeless patients in Canadian emergency departments

S.W. Kirkland, MSc, R. Chetram, G. Cummings, MD, B. Voaklander, T. Nikel, A. Davidson, B. Holroyd, E. Cross, C. Villa-Roel, K. Crick, S. Couperthwaite, BSc, D. Voaklander, PhD, B.H. Rowe, MD, MSc, University of Alberta, Edmonton, $\mathrm{AB}$

Introduction: Some Emergency Department (ED) presentations are considered convenience visits and potentially avoidable with improved access to primary care services, especially in marginalized groups (e.g., homeless, Aboriginal groups, etc). This study explored reasons for patient presentations at three urban EDs in Edmonton, Alberta focusing on the homeless population. Methods: Patients $>17$ years of age were randomly selected from electronic registration records at three urban ED sites and asked to complete a questionnaire. The questionnaire collected information on demographics, reasons for presentation, primary care visit history and abortive actions taken prior to presentation. Homelessness was self-identified and verified from address information at presentation. Results: Overall, 1408 patients completed the survey, of which $42(3.0 \%)$ were identified as homeless. Fewer homeless patients reported having a family physician (crude OR $=0.5$; 95\% CI: $0.3,0.9$ ); however, the attempts to access alternative care prior to the ED were similar between homeless vs. non-homeless patients $(44.7 \%$ vs. $53.2 \%)$. Homeless patients were more likely to present with an injury $(75 \%$ vs. $49.6 \%)$ and reported more minor (crude $\mathrm{OR}=3.2 ; 95 \%$ CI: $1.7,6.0$ ) and severe (crude OR $=3.5 ; 95 \%$ CI: 1.8 , 7.2) previous head injuries than non-homeless patients. Conclusion: Patients with unstable housing/homelessness are more likely to report a history of head injury, have life style choices that contribute to their poor health and are disconnected from the primary care providers who could address their complex health needs compared to non-homeless patients. More research is needed to understand the relationship between homelessness and head injury/concussions.

Keywords: emergency department overcrowding, homeless, brain injury

\section{MP35}

Does the modality of emergency department discharge instructions improve patient follow-up with a family physician?

S. Odorizzi, BSc, A. Stone, BSc, M. Klingel, MSc, S. Cruise, BSc, S.L. McLeod, MSc, R. Sedran, MD, MSc, BESc, University of Western Ontario, London, ON

Introduction: Many patients who present to the emergency department (ED) are discharged with instructions to follow-up with their family physician (FP) for continuation of care. Despite this, patients often do not see their FP. Written ED discharge instructions may enhance communication and lead to improved follow-up as compared to verbal instructions alone. The objective of this study was to determine if the modality of ED discharge instructions impacted follow-up with FPs. Methods: This was a prospective cohort study of adult patients presenting to a tertiary care ED (annual census 65,000 ) with a Canadian Triage and Acuity Scale (CTAS) score of 2-4 and were discharged home from the ED with instructions to follow-up with their FP. Patients were excluded if they did not have a FP or were unable or unwilling to consent. Patients were enrolled into one of 3 study arms: i) standard verbal discharge instructions (group 1), ii) standard verbal discharge instructions plus a written ED discharge summary form (group 2), iii) standard verbal discharge instructions and a written ED discharge summary form, and a copy of the ED discharge form was faxed to their FP (group 3). Trained research personnel contacted participants for a telephone interview after two weeks to ascertain whether they saw their FP. Results: 347 patients were screened for eligibility. 
115 were excluded, leaving $82(35.3 \%)$ in group 1, $61(26.3 \%)$ in group 2 and 89 (38.4\%) in group 3. Patient characteristics and demographics were similar across all three groups. $176(75.9 \%)$ had complete follow-up data. $41(50.0 \%)$ of patients in group 1 followed up with their family physician, compared to $39(63.9 \%)$ in group 2 and 49 $(55.1 \%)$ in group 3 . There was no difference found between group 2 and group $1(-13.9 \%, 95 \%$ CI: $-29.1,2.5)$ or between group 3 and group 1 ( $-5.1 \%, 95 \%$ CI: $-19.6,9.7)$. Among patients who did follow up with their FP, the median (IQR) number of days to the appointment was $9(3,13)$. The most common reasons for not seeing their FP included claiming to feel better $(31.9 \%)$, planning on making a future appointment (19.1\%) and patient stating their FP is inaccessible $(17.0 \%)$. Conclusion: Despite varying the type of ED discharge instructions given to patients, follow up with FPs remained low. Future studies should attempt to identify what ED information should be relayed to the FP and determine what method of discharge summary would be most useful for continuity of patient care.

Keywords: follow-up, discharge instructions, continuity of patient care

\section{MP36}

Incidence of neurogenic shock in the emergency department following acute traumatic spinal cord injury

D. Huang, MD, P. Oxciano, MD, D. Yan, MD, S. Harkema, PhD, A. Krassioukov, MD, PhD, Department of Emergency Medicine, Vancouver General Hospital, Vancouver, BC

Introduction: Cardiovascular abnormalities during acute period of spinal cord injury (SCI), including neurogenic shock are common manifestation of autonomic nervous system dysfunction. Unfortunately, we do not have a clinical picture of the expected time course of recovery of blood pressure parameters among newly injured individuals. The primary goal of this study is to investigate neurogenic shock after traumatic SCI, including the natural progression and degree of recovery of cardiovascular function after the acute period following SCI. Methods: A retrospective chart analysis of all cases admitted with acute SCI to the primary provincial neurosurgical centre in $\mathrm{BC}$ during a three-year period. Only adult subjects with acute traumatic SCI presenting to the tertiary care centre emergency department (ED) within 48 hours of injury were included. Data on demographics, level of injury, pharmacological management, and cardiovascular parameters were extracted from the time of injury and then daily for a period of one month. Results: A total of 225 patients presented to the provincial trauma centre ED during the study period, of which 87 patients met the inclusion criteria. 26 patients $(30 \%)$ presented with or developed neurogenic shock in ED (defined by SBP $<$ $100 \mathrm{mmHg}, \mathrm{HR}<80 \mathrm{BPM})$. The majority of these patients ( $85 \%)$ sustained cervical SCI, and 15 patients (58\%) had complete injuries (AIS A). 23 patients required vasopressor support range from 1 to 17 days, with 14 cases requiring vasopressor on day one. An additional group of 9 patients with multi-trauma also required vasopressor support on day one following SCI. In this group, 6 patients had cervical SCI, and 8 subjects had complete injuries (AIS A). Conclusion: Appreciation of cardiovascular dysfunction after SCI is crucial for the management of neurogenic shock in the ED. Our study demonstrated that approximately $30 \%$ of admitted cases of acute traumatic SCI presented with neurogenic shock. The majority of this group $(85 \%)$ sustained severe cervical SCI. Although the SCI were homogenous with respect to level and completeness of injuries, there was significant variation in duration of need for vasopressor therapy. There is a possibility that the extent of damage to spinal autonomic circuits varies significantly with SCI. Our work and future studies are warranted for the development of provincial guidelines and protocols.
Keywords: neurogenic shock, spinal cord injury, epidemiology

MP37

Perceptions and motivations of emergency medicine residents regarding diagnostic testing: a qualitative study

F. Zaver, MD, L.S. Wen, MD, MSc, George Washington University Hospital, Washington, DC

Introduction: Studies from the Institute for Healthcare Improvement estimate that $30 \%$ of diagnostic tests done are unnecessary. Little is known about trainees' perceptions of test ordering. We seek to understand Emergency Medicine (EM) residents' perceptions and motivations for ordering diagnostic testing. Methods: The study population was four classes of EM residents at George Washington University. All 43 residents were included, with a response rate of $100 \%$. We employed a 9-item questionnaire with a combination of closed and open-ended questions. Both in-person and phone interviews were conducted solely by the first author, an EM resident trained by the second author, an expert in narrative interview. Data were analyzed using grounded theory methodology, with $100 \%$ agreement between the authors. Results: Most residents (73\%) perceived emergency physicians (EPs) to order too many tests, $27 \%$ believed just enough, and none too few. The most common reasons for ordering tests were fear of missing emergent conditions, fear of malpractice, and patient expectations. $16 \%$ of residents discussed diagnostic testing with patients most of the time, $72 \%$ some of the time, $5 \%$ all the time, and $7 \%$ none of the time. Nearly all respondents $(98 \%)$ stated that they received no formal training in residency on how to involve patients in decisionmaking. The majority perceived themselves to order the same number of tests $(70 \%)$ as their peers; $23 \%$ perceived ordering fewer and $7 \%$ more. Conclusion: EM residents perceive EPs to order too many diagnostic tests, though they tend to self-report that they order the same number of tests as their peers. While patient expectation is cited as a major reason for ordering tests, most residents discussed testing with patients only some of the time. Incorporating shared decision-making into the residency curriculum may increase patient involvement, guide more tailored workup, and reduce unnecessary testing.

Keywords: clinical-decision making, health policy, resident trainee

MP38

Determinants of emergency physician productivity and utilization G. Innes, MD, A. McRae, MD, D. Wang, MSc, L. Mercuur, MD, G. Curry, MD, E. Lang, MD, University of Calgary, Calgary, AB

Introduction: Emergency physician (EP) productivity and utilization behaviour are important determinants of ED function, and they are highly variable. Our hypothesis was that training path (CCFP-EM vs. FRCPC), physician age and gender would be associated with differences in productivity (patients-per-hour) and utilization behaviour (admission rates, ED length of stay and imaging use). Methods: All EPs in Calgary belong to an integrated city-wide department that provides all regional emergency care at 3 sites. We prospectively capture MD performance parameters, including patients seen per-hour, admission rate within CTAS 3, MD throughput time (from sign-up to patient discharge) and imaging utilization. These data were electronically collated for the $141 \mathrm{ED}$ physicians who treated more than 300 patients in 2012. A descriptive analysis was performed. t-tests were used to determine the statistical significance of observed differences in parametric numeric data, and Wilcoxon rank sum tests for non-parametric data. Pearson correlation coefficients were calculated to assess the relationship between normally distributed numeric data. Results: During the study period, 108 male and 33 female EPs, $45 \%$ FRCP trained, treated 250,000 patients ( $83 \%$ CTAS 1-3). Median patients-per-hour was 2.02 
(IQR, 1.83-2.27); median CTAS 3 admission rate was $16.3 \%$ (IQR, 14.6-18.3) and median CTAS 3 throughput time was 1.75 hours (1.42.0). There were no differences in acuity mix based on training path, physician age or gender. Females worked $69 \%$ as many hours as males and saw slightly fewer patients per hour $(=0.2 ; 95 \% \mathrm{CI}, 0.05$ $0.35 ; \mathrm{p}=0.009$ ). Median CTAS 3 admission rates and throughput times varied by training pathway. FRCP physicians admitted $17 \%$ (IQR, 15$20)$ vs. $16 \%(14-17)$ for CCFP-EM physicians $(p=0.014)$. Median throughput time was 1.7 hours (1.4-1.9) for CCFP-EM and 1.8 hours (1.6-2.1) for FRCP physicians $(\mathrm{p}=0.02)$. We found no other significant differences in admission rates, throughput times, imaging rates or $72-$ hour bounce-back rates based on training path, physician age or gender. Physicians who worked more shifts (median split) tended to see more patients per hour (2.14 vs 2.02), admit fewer patients (16 vs. $17 \%$ ) and have shorter processing times (1.7 vs. 1.8 hours) but none of these differences were statistically significant. Notably, physician age did not correlate with productivity or utilization outcomes. Conclusion: In this setting, female gender was associated with slightly lower productivity (patients-per-hour) while CCFP-EM training was associated with slightly lower admission rates and throughput times. However, productivity and utilization differences, stratified by training path, physician age and gender, were very small and unlikely to drive meaningful differences in ED operational efficiency.

Keywords: emergency services, utilization, efficiency

\section{MP39}

Return to the ED after discharge following minor injury: results from 1436 patients in the Canadian Emergency Trauma Initiative (CETI) observational cohort study

J.S. Lee, MD, MSc, M. Sirois, PhD, X. Neveu, MSc, J.J. Perry, MD, MSc, A. Worster, MD, R. Daoust, MD, E. Lang, MD, M. Emond, MD, MSc, University of Toronto, Toronto, ON

Introduction: Older ED patients discharged following seemingly minor injuries are at higher risk for loss of function, but their return to the ED (RTED) is poorly described. Methods: OBJECTIVES: To describe: 1) RTED by previously independent seniors after ED discharge following a minor injury; 2) predictors of RTED at 3 and 6 months; and 3) discharge care and follow-up in this population. STUDY DESIGN: Prospective observational cohort study at 7 Canadian teaching EDs. PARTICIPANTS: Eligible patients were aged $\geq 65$ years, independent in 7 basic activities of daily living, assessed in an ED for a minor injury and discharged home within 48 hours. DATA COLLECTION: We assessed demographics, comorbidities, cognition using the previously validated Montreal Cognitive Assessment scale (MOCA), Identifying Seniors at Risk (ISAR) scale, and frailty index on eligible consenting patients at baseline. We then conducted followup telephone interviews confirmed by hospital chart reviews at 3 and 6 months to assess subsequent RTED. Polynomial Logistic regression analyses was used to identify predictors of RTED. Results: We recruited 1436 participants with a mean age of 77.1 years $-33.5 \%$ were male. Injuries included contusions (44.7\%), lacerations (26.2\%), fractures $(25.2 \%)$, trivial or mild head injuries $(18.0 \%)$, and sprains (12.7\%). The rate of RTED at 3 and 6 months post-injury were $15.2 \%$ (95\% IC: $12.8 \%-18.1 \%$ ) and $13.1 \%$ (95\% IC: $11.0 \%-15.5 \%$ ) respectively, adjusted for hospital clustering. The following 3 variables model best predicted RTED at three and six months: ISAR Serious memory problem, OR=2.97 (95\% IC: 1.26-7.02), living alone without help, OR=1.56 (95\% IC: 1.10-2.22), and MOCA scores < 23/30, $\mathrm{OR}=1.48$ (95\% IC: $1.03-2.14)$. Analgesia was the most frequent type of care provided to injured seniors $(35.3 \%)$. Discharge planning was limited to referrals to family physicians $(13.5 \%)$. Conclusion: Up to $21.9 \%$ of seniors who were independent prior to a minor injury needed to RTED by 6 months. Living alone and cognitive impairment increased RTED. Future study should look at targeted interventions to reduce the need for RTED.

Keywords: geriatrics, functional decline, emergency department

MP40

Physician self awareness of CT ordering practice

A. Kadhim-Saleh, BHSc, MSc, J. Worrall, MD, M. Gatien, MD, M. Taljaard, PhD, J.J. Perry, MD, MSc, University of Ottawa, Department of Emergency Medicine, Ottawa, ON

Introduction: Studies have demonstrated wide inter-physician variation in resource utilization, including the use of Computed Tomography (CT). High rates of CT ordering may increase cost and expose patients to unnecessary radiation, contrast, and further tests. Our objectives were to measure emergency physician (EP) $\mathrm{CT}$ ordering rates and awareness of their CT utilization Methods: A retrospective review of an imaging database at two Emergency Departments of a tertiary care adult teaching hospital was conducted in January, March, June, and September 2012. Departmental billing records were used to determine numbers of patients seen per EP. Physicians completed a paper survey to estimate their rate of ordering, and to assess, on a 5-point Likert scale, their beliefs about their ordering compared to colleagues. Data were analyzed using descriptive statistics. Results: Complete CT ordering and billing data were obtained for 59 EPs. EPs saw 45,834 patients, and ordered $6,513 \mathrm{CTs}$, a mean ordering rate of $14.2 \%(95 \% \mathrm{CI}, 13.9 \%-14.5 \%)$. The ordering rate ranged from $5.9 \%$ to $25.9 \%$ (median rate $14.9 \%$, interquartile range 10.9-16.9\%). 45 EPs $(76.3 \%)$ completed the survey. Mean perceived ordering rate was $12.6 \%$, and was weakly correlated with actual ordering $(\mathrm{r}=0.19, \mathrm{p}=0.21) .32 \%$ of EPs underestimated their absolute CT ordering by at least 5\% (Exact 95\%CI: 19.5- 46.7\%). 18\% of EPs over-estimated their absolute CT ordering by at least 5\% (Exact $95 \%$ CI: $8.2-32.7 \%$ ). Only $29 \%$ of EPs were able to predict their ordering behaviour to the correct quintile. Three EPs $(6 \%)$ believed they order "many fewer" CTs than their peers, 12 (24\%) "fewer", 32 (63\%) "about the same", 4 (8\%) "more", and 0 "many more". Of the 17 EPs in the two highest ordering quintiles, only 3 (18\%) knew they were high orderers, and $71 \%$ underestimated their ordering rate. Of the $17 \mathrm{EPs}$ in the two lowest ordering quintiles, $8(47 \%)$ knew they were low orderers, and $8(47 \%)$ underestimated their ordering rate. Conclusion: There is large variation in CT ordering amongst EPs. Physicians' self-reported ordering rate correlates poorly with actual ordering. Low orderers tended to underestimate their ordering; high orderers tended to underestimate. High CT orderers were rarely aware that they ordered more than their colleagues.

Keywords: imaging, utilization, practice variation

\section{MP41}

The medical education in cases series: online faculty development for medical education

T.M. Chan, MD, B. Thoma, MD, MA, M. Lin, MD, MedEdLIFE.org Research Collaboration

Innovation concept: Since Salman Khan's online lectures took the world by storm, disruptive innovations have begun changing the landscape of medical education. These innovations have been mainly aimed at medical learners with few resources aimed at developing medical educators in emergency medicine. This innovation demonstrates that educators can be engaged to participate in an online faculty development exercise. Methods: In July of 2013, the Academic Life in Emergency Medicine (ALiEM) website launched the Medical Education in Cases Series (MEdICS), a set of articles with a structural framework based loosely on the cases in the Harvard Business Review. Each 
month fictionalized scenarios are used to start an online discussion about clinically- and educationally-relevant dilemmas. The cases were intentionally written with multiple layers of complexity so they would resemble real life situations. They are released online and promoted using Twitter, Facebook, and Google Plus. Participants make comments on Twitter and the blog comment section. One week later a curated digest of the community comments is posted alongside expert commentaries written by experienced clinicians. Curriculum, tool, or material: We launched 5 cases between July and December that emphasized different CanMEDs and ACGME roles. During the week after the case was posted, each case received on average 866.8 pageviews from 317 cities and 40 countries, 748.0 unique visitors. 33.0 comments (18.6 excluding commentators). 51.8 Tweets, 20.2 Facebook likes. and 5.6 Google+ "+1's". Conclusion: Our innovation is proof of concept that emergency medicine educators can be engaged in online professional development. More innovation and research is required to better understand how this strategy may be used to augment classic methods of faculty development.

Keywords: innovations in EM education, social media, blogging

\section{MP42}

Development of a comprehensive simulation curriculum for emergency medicine residency training programs

C. Filipowska, MD, PhD, A.H. McConnell, BSc, MKin, MD, S.L. McLeod, MSc, J.R. Frank, MD, K. Woolfrey, MD, University of Toronto, Toronto, ON

Innovation concept: Residency training can be defined as the acquisition of a consummate skill-set consistent with that of a competent emergency physician. Despite the absence of uniform curriculum for competency-based training in emergency medicine (EM), expectations of trainees are expanding. In response to this, many EM training programs have increased their use of simulation training to supplement current didactic and experiential curriculum. The objective is to derive a standardized, comprehensive national simulation curriculum for emergency medicine and to provide the EM program directors with an instructional tool to cover topics/skills that may be optimally delivered via simulation training. Methods: 52 Canadian stakeholders (defined as an expert in the area of medical education, simulation, or clinical $\mathrm{EM})$ at the $13 \mathrm{EM}$ sites across the country will be invited to participate in an online Delphi methodology to assess the Royal College Objectives of Training (OOTs outlined by the Royal College in 2008, revised 2011) document for each objective's individual suitability for inclusion in a comprehensive national simulation curriculum for Emergency Medicine. Participants will assess each OOT and rate whether it is best taught by simulation. Consensus will be achieved with $70 \%$ agreement. If an objective does not attain agreement, it will be included in a subsequent iteration to a maximum of three iterations. Curriculum, tool, or material: This project will address the competency-based educational void in EM residency training by defining a novel, comprehensive national simulation curriculum. The future application of this project will be generation of the simulation curriculum itself. Not only will this encourage collaboration amongst experts across Canada, but it will also provide an online manual to all 13 program directors for curriculum implementation. Conclusion: This will be a working body, as its inherent design will allow for future validation of derivation as well as improvement on scenarios based on educator feedback.

Keywords: innovations in EM education, simulation, consensus/ Delphi

MP43

'Telesimulation' in emergency medicine: connecting Canadian faculty to Ethiopian residents to provide procedural teaching
E. Austin, MD, M. Landes, MD, MSc, N. Meshkat, MD, P. Miller, MD, J. Maskalyk, MD, A. Okrainec, MD, A. Azazh, MD, University of Toronto, Toronto, ON

Innovation concept: Simulation is a prevalent training method in Canada for teaching technical skills in emergency medicine. In resource-limited settings, challenges to simulation training often include a lack of equipment and/or qualified trainers. 'Telesimulation' is a low-cost and validated tool that remotely connects teachers with trainees using the Internet and re-creates a similar environment to simulation in which the teacher and trainees are in the same room. Methods: In 2010, Addis Ababa University launched Ethiopia's first emergency medicine residency program, supported in part by the University of Toronto (UofT). In 2012, a telesimulation lab was setup at Tikur Anbessa Hospital, Addis Ababa, to connect with the existing lab at the Telemerty/Chang Telesimulation Centre in Toronto. Each telesimulation lab contains a simulation "box" where one set of webcams allow visualization of hands and procedural equipment, and a second direct visualization of each person using the simulator. Cameras are connected using SkypeTM software, allowing both the instructor and trainee to simultaneously see the contents of each other's simulation box and to communicate. Curriculum, tool, or material: To date we have developed and implemented a teaching module for central venous catheter insertion using telesimulation. Each course consists of a thirtyminute didactic lecture over SkypeTM, a one-hour skills training session with 2-3 participants, and 15 minutes per participant of 1:1 training with the instructor. Participants are evaluated at the end of the session for skill competency and provided feedback. Conclusion: Telesimulation has been used to train surgeons worldwide in laparoscopy (1) and in Botswana for pediatric intraosseous insertion (2). This project provides a method, telesimulation, to remotely connect teachers from UofT with EM trainees in Ethiopia to develop procedural skills. Telesimulation alleviates the time and cost of travel for teachers while still providing an excellent educational opportunity for trainees. Future directions of this project involve teaching more procedures (ie. pericardiocentesis and burr holes), performing a validation study of this teaching method in comparison to in-country teaching, and developing the site in Ethiopia as a centre to provide further telesimulation to other more remote sites. . (1) Okrainec A, Henao O, Azzie G. Telesimulation: an effective method for teaching the fundamentals of laparoscopic surgery in resource-restricted countries. Surg Endosc 2010 Feb;24(2):417-22. (2) Mikrogianakis A, Kam A, Silver S, Bakanisi B, Henao O, Okrainec A, et al. Telesimulation: an innovative and effective tool for teaching novel intraosseous insertion techniques in developing countries. Acad Emerg Med 2011 Apr;18(4):420-427.

Keywords: innovations in EM education, internation medicine, simulation

\section{MP44}

Tactical emergency medicine care in a military medicine, law enforcement, and emergency medicine collaborative training program

J.M. Goodloe, MD, A.O. Arthur, PharmD, W. Justice, K. Massie, S.H. Thomas, MD, MPH, The University of Oklahoma School of Community Medicine, Tulsa, OK

Innovation concept: Emergency physicians are increasingly providing medical oversight and response support to law enforcement agency special operations teams. The dynamics and challenges of tactical emergency medicine differ substantially from traditional EMS and emergency medicine practices. Focused tactical care training for emergency medicine resident physicians and EMS fellows is integral to success and safety in their future medical oversight of such programs. Methods: A multi-disciplinary faculty, comprised of active duty spe- 
cial operations military medical providers, law enforcement officers, and emergency medicine physicians with prior EMS and tactical training, deliver a four-day curriculum in tactical emergency medicine. The curriculum includes the didactics of the tactical combat casualty care curriculum (TCCC) taught on a law enforcement academy campus. Practical exercises include basics of tactical operations movements, remote patient assessment ("medicine across the barricade"), personal defense tactics, and medical care in sensory overload and sensory deprivation scenarios. All education leads to the culminating lab of a live animal tissue exercise in which goats, under the constant supervision of a veterinarian, sustain a variety of penetrating wounds, requiring time-critical interventions to sustain life. The live tissue exercise is conducted in a tactical field exercise set-up. The animals are expected to live through several hours of active resuscitation. The care provided is done in care under simulated fire, casualty collection point, and tactical evacuation paradigms. Curriculum, tool, or material: Multiple physicians have been successfully trained in this collaborative program. Pre-test and post-test scores and exit interviews indicate substantial didactic and clinical knowledge acquisition. Consistently successful live animal resuscitation and ongoing support from significant airway, thoracic, abdominal/pelvic, and/or extremity trauma directly correlate to appropriate clinical assessment and procedural skills being delivered in realistic simulated environments to include active gunfire, explosions, and chemical threats. Conclusion: A multi-disciplinary faculty of military medical operators, law enforcement officers, and emergency physicians can translate the didactic and clinical knowledge of tactical emergency medicine to emergency medicine residents and EMS fellows.

Keywords: innovations in EM education, tactical emergency medicine, emergency care in austere environments

\section{MP45}

EM clinical teaching teams: a novel longitudinal resident teaching and assessment program

K. Sampsel, MD, S. Choi, MD, J.R. Frank, MD, University of Ottawa, Ottawa, ON

Innovation concept: Shiftwork as the basis for clinical emergency medicine (EM) creates challenges in the teaching and supervision of trainees. With expanding numbers of residents as well as faculty, an individual trainee may work with a different staff on every shift, hampering a longitudinal view of learning and performance, a phenomenon called "educational tourism". Teaching and assessment for an individual resident is enhanced when faculty are familiar with their progression of competence and areas of weakness. At the UOttawa Department of Emergency Medicine, we developed the Clinical Teaching Teams (CTT) program to address this need. The CTT was designed to enhance the learning experience of residents during their EM blocks by pairing them with distinguished teachers who would be able to assume a greater sense of ownership over the progress of their residents and be able to tailor their teaching, mentorship and guidance. Methods: All 62 of the staff physicians and 45 of the Royal College EM program residents at the University of Ottawa were invited to voluntarily participate in this program. Teams of three staff physicians were matched with a resident by the residency program directors to create a variety of staff physicians' experience, interests, training, teaching awards, resident evaluations and resident feedback. While all residents were eligible, preference was given to senior residents. During residents' emergency medicine blocks, they were preferentially scheduled to work approximately one third of their shifts with members of their CTT. Curriculum, tool, or material: CTT members established an individualized learning plan for the academic year including on-shift discussion topics, reading materials and practice oral examinations. Tailored feedback was given to the resident on the daily encounter evaluations.
Individual learning strategies were implemented by the CTT to address identified weaknesses in a resident's performance either clinically or academically, including direct observation, targeted clinical encounters and practice written and oral examinations. Additionally, a narrative of how learning goals were being met was forwarded by email semiannually by the CTT members to the residency program directors. Conclusion: The CTT program was launched as a pilot project in 2012, expanded in 2013, and was well received by both staff and residents. The CTT program model allows for a more consistent and individualized approach to teaching and performance appraisal for emergency medicine residents within the constraints of expanding numbers of staff and residents and the vagaries of shiftwork scheduling. Academic or clinical areas of weakness can be identified more rapidly and addressed in a tailored manner by both the CTT and the residency program directors. This allows for more meaningful assessment of performance for both the resident and residency program directors.

Keywords: innovations in EM education, clinical teaching, mentoring

\section{MP46}

The social media index: curating emergency medicine blogs and podcasts

B. Thoma, MD, MA, J.L. Sanders, MD, PhD, Q. Paterson, J.B. Steeg, BSc, M. Cadogan, MD, M. Lin, MD, Massachusetts General Hospital, Boston, MA

Innovation concept: An explosion in emergency medicine and critical care (EMCC) blogs and podcasts has made it difficult for readers/listeners to find good content. Alternative metrics (altmetrics) that use social media are increasingly accepted parameters in academia. The Social Media Index (SMi) was developed to rank blogs/podcasts using altmetrics. Methods: 245 active EMCC websites were found using prospective snowball sampling between 2002 and 2013 and a Boolean search in November 2013 using the terms: ("emergency medicine" OR "critical care" OR "intensive care") AND (podcast OR blog). Websites included were: blogs or podcasts, freely accessible, related to EMCC, composed in English, active within the prior 6 months, and not hosted on an institution's or journal's website. All data was updated December 29, 2013. The SMi was created with components that provide data on web traffic (Alexa Rank and PageRank) and social media followership (Twitter, Google+ and Facebook). Three formulas were derived using raw, rank-order and log transformed versions of the data. In all formulas each component was given equal weight by normalizing the component score on a scale of $0-2$. The five components were added to score each site ( $\max$ score 10 , minimum score 0 ). We calculated descriptive statistics for SMi variants and Pearson correlations between variants. The correlation between the log SMi and years since website creation was calculated to test if the SMi was associated with website age. The rankings will be published on the Academic Life in Emergency Medicine blog. Curriculum, tool, or material: 165 websites met inclusion criteria. Mean (standard deviation) and median (interquartile range) score for SMi variants were: raw $1.2(1.0)$ and 1.0 (0.9), rank 4.2 (2.3) and 4.0 (3.2), $\log 3.9$ (1.8) and 4.0 (2.1). Pearson correlations were: raw-rank 0.76 , raw-log 0.78 , rank-log 0.95 (all $\mathrm{P}<0.01$ ). Correlation between $\log \mathrm{SMi}$ and website age was 0.21 . The top three websites on the log SMi were Life in the Fast Lane, EMCrit and Academic Life in EM. Conclusion: Five publicly available metrics were used to develop a formula to differentiate EMCC websites. SMi variants correlated strongly and produced similar website rankings. The SMi was not strongly correlated with website age, suggesting website significance is not strongly confounded by age. As more rigorous assessments of quality are developed, we will determine whether the SMi is an effective surrogate for website quality.

Keywords: innovations in EM education, social media, medical education 
MP47

Exposing Canadian medical students to point-of-care ultrasound: an undergraduate ultrasound symposium

R. McLarty, BSc, W. Yan, BSc, N. Packer, BSc, C. Byrne, BSc, D. Thompson, MD, R. Arntfield, MD, University of Western Ontario, London, ON

Innovation concept: There is increasing interest for point-of-care ultrasound (PoCUS) in undergraduate medical education. However, training opportunities inevitably vary between institutions. The objective of this study was to develop an introductory PoCUS educational program accessible to medical students across Canada. Methods: A two-day, objective-based curriculum in ultrasound education was developed in collaboration with local physician expertise. Registration was promoted and opened to medical students across Canada. Student perspectives on the clinical and educational value of PoCUS were assessed with a seven-point Likert scale. Curriculum, tool, or material: Thirty-five medical students from five Canadian medical schools participated. Twenty students from an additional three institutions registered on a waitlist. The curriculum included an introductory lecture on ultrasound fundamentals and six hands-on ultrasound workshops: cardiac; lung and pleura; abdominal aorta, hepatobiliary and renal; focused assessment with sonography for trauma; obstetric and gynecologic; and procedural guidance. Sessions were 90 to 120 minutes in length and coordinated by physician experts. Each workshop consisted of approximately 20 minutes of didactic learning followed by scanning time at a ratio of four participants to one instructor. The curriculum ended with participants applying newly acquired skills in a fun and challenging 'Sono Games' competition. Participants strongly agreed that the focused two-day exposure on PoCUS enhances their understanding of anatomy and clinical medicine. Conclusion: A two-day, objective-based curriculum provides medical students with efficient, hands-on extracurricular exposure to PoCUS. This Canadian first represents an engaging student-physician collaborative model that can be implemented elsewhere.

Keywords: innovations in EM education, ultrasound, medical students

\section{MP48}

An emergency physician multi-measure performance profile for promoting reflections on practice and promoting regression to the mean through peer comparison

G. Curry, MD, G. Innes, MD, A. McRae, MD, D. Wang, MSc, L. Baker, MD, L. Mercuur, MD, K. Lonergan, MSc, T. Rich, MD, E. Lang, MD, University of Calgary, Calgary, AB

Innovation concept: Variability in emergency physician (EP) practice is expected, but excessive variability may be a marker of a struggling (or outstanding) physician. We leverage innovations in the reporting of administrative and operational data to create dashboards that provide key performance metrics for EPs with insight as to how their practice profile compares with their peers. Our objective is to describe EP practice variability on four key clinical and operational performance metrics. Methods: Calgary's four emergency departments use common patient care information and physician order entry systems that link patient demographics, triage data, operational time elements, diagnostic utilization and treatment data to individual patient-physician encounters. We used data visualization software $\left(\right.$ Tableau $\left.{ }^{\circledR}\right)$ to develop a comprehensive dashboard that can be used to summarize each EP's performance. These dashboards are made available to EPs for comparison against peers and as part of regular performance reviews. Curriculum, tool, or material: Dashboards were created for 160 emergency physicians who work across 3 tertiary care facilities that manage a total 20000 visits annually. Physi- cians can view and compare their performance for the metrics of: 1 3 -day revisit rate resulting in readmission, reflecting safety and clinical acumen. 2. Clinical productivity in patients seen per hour, reflecting workflow efficiency. 3. Length of stay for CTAS 3 patients, reflecting efficacy of decision-making and 4. Diagnostic imaging utilization, which reflects appropriate stewardship of precious resources. All metrics reveal marked variation in our physician group with 10 or more physicians exceeding the 90th percentile for each metric. Conclusion: We have identified significant variation in practice for both clinical and operational metrics within a large group of emergency physicians. Further work will focus on whether access to these profiles promotes regression to mean values and more uniform care delivery.

Keywords: innovations in EM education, Dashboards, Performance

MP49

Journal Club 2.0: collaboration between a traditional journal and an academic blog to create a global journal club experience T.M. Chan, MD, R.P. Radecki, MD, MS, S.R. Rezaie, MD, B. Thoma, MD, MA, M. Callaham, MD, M. Lin, MD, MedEdLIFE.org Research Collaboration

Innovation concept: Salman Khan and the Khan Academy have challenged medical educators to flip the classroom and rearrange the Flexnerian architecture of our education systems.. "Journal Club" documents in standard medical journals are often opinion or digest articles that analyze the papers for you. This one-way flow of information does not encourage critical appraisal of articles, and seldom provide a chance for readers to engage with the format.The efforts of most journals over the past decade to facilitate more timely engagement with readers with their articles (via comment and discussion), have been noticeably unsuccessful to date (being neither timely nor interactive). Methods: In the fall of 2013, the Annals of Emergency Medicine journal allied with the Academic Life in Emergency Medicine (ALiEM) blog to pilot a global journal club format. An openaccess journal article was selected to encourage discussion around evidence-based medicine and critical appraisal techniques. Questions were developed by the Annals team and posted on the ALiEM blog. Over the course of the week, the ALiEM team members (SR, RR, ML) facilitated the asynchronous discussions occurring on the blog and Twitter. The blog served as a 'home base' for participants to contribute longer answers, while Twitter (through the use of the hashtag \#ALiEMJC) allowed dynamic moment-to-moment discussions with multiple contributors. The discussion opening and closing date were announced a priori, and participants were invited to comment by the proposed closing date. Each question was placed on its own unique blog page, so that readers could post their responses to the questions in the comments of said blog post. Curriculum, tool, or material: Our Google Analytic Data shows that we had 1,171 page views of the material within two weeks of the initial posting, spanning 148 cities in 25 countries. There was an average of 254 hits $(S D=87)$ per question page of the journal club format. Twitter analytics revealed 63 unique Twitter contributors to the discussion, who collectively posted 142 tweets. The tweet reach, or number of unique Twitter accounts who saw any tweet related to \#ALiEMJC, was 33,202. Conclusion: This innovative new methodology harnesses the international reputation of two leading Emergency Medicine organizations (Annals of EM, ALiEM) to foster discussion in a mixed, new media format via blog posts and Twitter activity. This may serve as a possible new avenue for generating discussion between practicing physicians in different locales, and help with enhancing evidence based practices around the world.

Keywords: innovations in EM education, blogging, journal club 
MP50

Alternative care prior to emergency department visits in a Canadian urban setting

R. Chetram, M.B. Ospina, PhD, S.W. Kirkland, MSc, B. Voaklander, T. Nikel, A. Davidson, B. Holroyd, C. Villa-Roel, S. Couperthwaite, BSc, G. Cummings, MD, D. Voaklander, PhD, B.H. Rowe, MD, MSc University of Alberta, Edmonton, $\mathrm{AB}$

Introduction: When access to health services is required, the emergency department (ED) is often the only and best option available to patients. This study assessed the association between sociodemographic factors and attempts to access alternative care prior to ED presentation in an urban setting. Methods: A standardized questionnaire was administered to 1,408 randomly selected patients aged $>17$ years, with non life-threatening conditions in three EDs in Edmonton (Canada). Backward logistic regression explored associations among sociodemographic factors and seeking alternative care prior to the ED visit. Results: Among the $49.9 \%$ of patients who attempted alternative care before their ED visit, $32.5 \%$ visited a physician, $12.6 \%$ visited other care professionals, and $28.4 \%$ called a physician's office. The majority of those who called a physician's office $(67.5 \%)$ were advised to seek ED assessment. Of those who attempted accessing alternative care prior to visiting the ED, $90.9 \%$ decided that the ED was their best care option. Factors associated with not seeking alternative care prior to an $\mathrm{ED}$ visit were being male (odds ratio $[\mathrm{OR}]=0.7 ; 95 \%$ confidence interval [CI]: $0.5,0.9)$, having low educational level $(\mathrm{OR}=0.7 ; 95 \%$ CI: $0.6,0.9)$ and not having a family physician $(\mathrm{OR}=0.6$; $95 \% \mathrm{CI}$ : $0.5,0.8)$. Conclusion: Nearly $50 \%$ of patients attempt alternatives to the ED prior to their presentation; however, they are often directed to the ED and feel the ED is the most appropriate. Increased access to Family Physicians, while desirable, may not mitigate input issues in ED overcrowding.

Keywords: access to care, alternatives, emergency department overcrowding

\section{MP51}

Does an educational intervention increase radiation dose knowledge among physicians

M. Nauss, MD, J. Yang, PhD, Henry Ford Hospital, Detroit, MI

Introduction: Physician knowledge of the radiation dosages associated with imaging studies used in the Emergency Department is poor. Recently there has been an increase in articles on the link between cancer risk and radiation exposure. This study was undertaken to determine if an educational intervention can increase physicians' knowledge of radiation dosages, and if this increase correlates with the likelihood that a physician will discuss radiation with a patient or worry about a patient's radiation exposure. Methods: An anonymous survey was carried out among EM residents and staff at an academic center and three affiliated community ED's. Participants were assessed both at baseline and after the educational intervention was performed. This intervention consisted of a lecture and placement of prominent signs, with the correct dosages for 6 imaging studies, in each ED.These studies included: chest $\mathrm{x}$-ray, cervical spine $\mathrm{x}$-ray, CT head, CT chest, CT cervical spine, and $\mathrm{CT}$ abdomen/pelvis. After four months the survey was re-administered to the participants who had completed the initial survey. Answers within $25 \%$ of the actual radiation dose were considered correct. This determination was made a priori and was based on a previous study that used the same criteria due to the difficulty of physicians to recall exact radiation dosages. Results: 78 physicians completed the initial survey while 58 completed the subsequent one. Five in the pre survey and three in the post survey did not give a full set of answers in the radiation dosage section and were dropped from analysis. All physicians in the pre survey did complete the likelihood/discussion portion of the ques- tionnaire with only one physician not providing a full set of answers for this section in the post intervention survey. There was a small increase in the proportion of physicians whose answers were correct in 5 of 6 imaging test. This increase reached statistical significance for 2 of 6 imaging studies: CT chest,CT abdomen/pelvis. There was also a small, but not significant, increase in physicians who discuss dosages with their patients and who worry about their patients' radiation exposure . Conclusion: Our educational intervention led to a small increase in physician knowledge of radiation dosages. This increase did not lead to a significant change in discussion of the risks of radiation with patients nor physician worry about exposing patients to radiation.

Keywords: imaging, computed tomography, education

MP52

To CT, or not to CT? The utilization and diagnostic role of computed tomography amongst obese pediatric appendicitis patients H. Roy, M.A., B. Burbridge, MD, University of Saskatchewan, College of Medicine, Saskatooon, SK

Introduction: Appendicitis is a leading cause of pediatric ED visits. However, obesity is an independent risk factor for a non-diagnostic ultrasound $(\mathrm{U} / \mathrm{S})$, and childhood obesity rates are a growing concern. Desire to limit pediatric radiation exposure (via Computed Tomography [CT]) has led the Canadian Association of Radiologists to recommend that when $\mathrm{U} / \mathrm{S}$ is non-diagnostic and clinical suspicion is high, consideration be given to treatment without further imaging (i.e. CT). This study evaluates this option and fills a current research void by quantifying the influence of CT on the clinical management of pediatric appendicitis. Methods: A 2-year retrospective case series including all children 0-18 years presenting to an ER with suspected appendicitis (SA), divided into obese $(\mathrm{O})$ ( $\geq 85$ Body Mass Index for Age Percentile [BMIFAP]) and non-obese (NO) ( $<85$ BMIFAP) groups, comparing how often they received a $\mathrm{CT}$, why they received it, and what influence it had on diagnosis and treatment. Results: Of 223 pediatric patients with SA, 54 $(24.2 \%)$ received CT. Obese patients were more likely to receive a CT $(28.9 \% ; n=84)$ than NO patients $(21.6 \% ; n=139)$. The most common reason why both groups underwent $\mathrm{CT}$ was non-diagnostic $\mathrm{U} / \mathrm{S}(\mathrm{O}=$ $75 \%$; $\mathrm{NO}=80 \%)$, followed by abscess investigation $(\mathrm{O}=16.6 \%$; $\mathrm{NO}=$ $20 \%$ ), and physician's choice due to extensive body habitus $(\mathrm{O}=8.3 \%)$. $65 \%$ of the CT scans administered after non-diagnostic U/S confirmed the preliminary diagnosis of appendicitis $(95 \% \mathrm{CI}=50 \%, 78 \%)$, but that number jumps to $80 \%$ when just obese patients are considered (NO $=50 \%$ ). Therefore, the odds that a CT scan will change the diagnosis of an obese pediatric patient with SA are $75 \%$ lower than for a NO patient $(\mathrm{OR}=0.25 ; 95 \% \mathrm{CI}=0.064,0.97 ; \mathrm{p}=0.039)$. Conclusion: Since a follow-up CT scan has only a $65 \%$ appendicitis confirmation rate, the decision to order a CT following a non-diagnostic U/S would expose the patient to potentially harmful radiation, but it would also be apt to lower the likelihood of a false positive. Therefore, physicians should make follow-up diagnostic imaging decisions for SA on a case-by-case basis, depending on their level of clinical suspicion. In so doing, they should recognize that obese patients are more likely than non-obese patients to have a follow-up CT that confirms their suspicion of appendicitis. As a result, a physician whose clinical suspicion is high may be less inclined to expose an obese pediatric patient with SA to the radiation dose from a CT.

Keywords: pediatrics, appendicitis, computed tomography

\section{MP53}

Mild traumatic brain injury: risk factors of short term post-concussion symptoms

P. Turgeon, X. Neveu, MSc, N. Le Sage, MD, MSc, J. Chauny, MD, MSc, M. Emond, MD, MSc, M. Ouellet, PhD, L. Moore, PhD, Centre de recherche du CHU de Quebec, QC 
Introduction: In all industrialized countries, traumatic brain injuries (TBI) are among the major public health problems, with a prevalence of 600 victims per 100000 people in Canada. A limited number of studies have been conducted on acute post-concussion symptoms and most of them were based on small samples or retrospective analyzes. The main objective of this study was to identify patients at risk of developing post-concussion symptoms during the 10 days following the trauma upon their initial assessment in the emergency department. The secondary objective was to estimate the prevalence of post-concussion symptoms during the first 10 days after a mild TBI. Methods: In this prospective cohort study, were eligible all patients aged 14 years or more, assessed in the emergency department (ED) of one of the five selected trauma centers in the Province of Quebec in the first 24 hours after injury, that did not require hospitalization and met the criteria of mild traumatic brain injury. Data were collected at the ED and by phone follow-up within 10 days following the trauma. The main outcome was measured using the Rivermead Post-Concussion Symptoms Questionnaire. For each factor, adjusted relative risk for the presence of symptoms was calculated with IC 95\%. Results: 406 patients were included for the analysis. The following factors increased the risk of symptoms during the first ten days after trauma : female gender (1.40 [1.19 to $1.65])$, history of depression or bipolar disease (1.39 [1.15 to 1.68$]$ ), confusion of 30 minutes or more after trauma (1.45 [1.22 to 1.71] ), headache in $\operatorname{ED}(1.35$ [1.16 to 1.58$]), \mathrm{GCS}<15$ in the $\operatorname{ED}(1.28$ [1.09 to 1.50$])$ and motor vehicle accident (1.32 [1.12 to 1.55$])$. Conclusion: This study identified some risk factors predicting the presence of short term post-concussion symptoms. Those latter can affect the return to work and sports during the first days after mild TBI. The identification of predictors could now help identifying patients at risk of such symptoms as soon as they visit the emergency department. Overall, knowledge of these risk factors will help cliniciens to provide more personalized recommendations for these patients.

Keywords: mild traumatic brain injury, post-concussion symptoms

\section{MP54}

Improving shiftwork: are casino shifts the answer?

H. Levin, MD, T.P. Lynch, MD, J. Seabrook, Ph.D, N. Poonai, MD, G. Sangha, MD, Children's Hospital: London Health Sciences Centre, London, ON

Introduction: Shiftwork is fundamental to the function of an emergency department. Two problems associated with shiftwork are working during sleep hours and sleeping during waking hours. This causes disruption of the body's circadian rhythm. There is extensive literature documenting the adverse effects of circadian rhythm disruption. The concept of anchor sleep suggests that being able to sleep part of each night at the same time can help regulate circadian rhythm. However, standard overnight shifts do not allow for this. Some emergency departments have implemented casino shifts that split the night shift in two; the split generally occurs at $0400 \mathrm{~h}$. Anecdotal reports of casino shifts are positive; however, there is a paucity of literature that evaluates this perceived increase in wellbeing. The objective of this study is to quantitatively evaluate the wellbeing of residents doing casino shifts versus standard overnight shifts while working in a Pediatric Emergency Department (PED). Methods: This is a randomized controlled study of residents rotating through the PED at Children's Hospital, London Health Sciences Centre from July 2013 and June 2014. Residents rotating through the PED are scheduled for traditional overnight shifts on odd numbered blocks or casino shifts on even numbered blocks. Residents complete 2 questionnaires assessing wellbeing, one at the beginning and one at the end of their PED block. The questionnaires are comprised of the brief resident wellness profile (BRWP) and 10 non-validated Likert-scale items. The BRWP is a validated tool to assess resident wellness. The 10 non-validated items were developed for this study to assess wellness related to shiftwork. Results: Preliminary results from 62 residents; 30 standard overnight and 32 casino shift were included in the analysis. There was no significant difference in the relative change in the BRWP between the standard overnight and casino shift groups ( $p=0.703$ ). There was also no significant difference in the 10 non-validated items between the standard and casino groups. Conclusion: There is no difference in the effect on resident wellbeing of working casino shifts compared to standard overnight shifts. This is contrary to anecdotal evidence and to a previous study that looked at the wellbeing of emergency room physicians scheduled for casino shifts. However, there are several limitations that make it difficult to isolate the effects of shiftwork on resident wellbeing

Keywords: casino shift, anchor sleep, shiftwork

\section{MP55}

Is ultrasound associated with a lower negative appendectomy rate in children suspected of acute appendicitis?

A. Vieira, MD, N. Chen, BScPhm, A. Leung, BSc, R. Lim, MD, G. Joubert, MD, N. Poonai, MD, Western University-Schulich School of Medicine and Dentistry, London, ON

Introduction: Appendicitis is one of the most common conditions requiring urgent abdominal surgery in children. Accurate clinical diagnosis in the pediatric population can be difficult with up to $20 \%$ of children receiving negative appendectomies. The use of ultrasound (US) has increasingly become the standard of care in establishing a diagnosis prior to surgery. However, US can lead to delays in management without clear advantages in diagnostic accuracy. The purpose of this work is to examine whether pre-operative US is associated with a decreased negative appendectomy rate among children who received an urgent appendectomy for suspected acute appendicitis. Methods: This was a retrospective chart review of children 0-18 years who had an urgent appendectomy from January 1, 2000 to May 31, 2013 for suspected appendicitis following clinical assessment and/or pre-operative US at Children's Hospital, London. Data was analyzed by SPSS version 19. Final pathology reports of patients with clinically suspected appendicitis (no US), inconclusive US (other diagnosis equally likely), normal US and US suggestive of appendicitis were compared to determine negative appendectomy rates. A subgroup analysis looking at laboratory indices of appendicitis (WBC count, CRP, and fever) was performed. A p-value less than 0.05 was considered significant. Results: A total of 1291 patients were included in the analysis; 727 had US suggestive of appendicitis, 106 were inconclusive, 45 with normal and 413 with no US. There was no significant difference in negative appendectomy rates in patients with US suggestive of appendicitis compared to patients with clinically suspected appendicitis $(9.4 \%$ vs. $9.2 \%, \mathrm{P}$ value $=0.932$ ). There was also no significant difference when comparing those with inconclusive US findings to clinically diagnosed patients $(16 \%$ vs. $9.2 \%, \mathrm{P}$ value $=0.068)$. Conclusion: There was no significant difference in negative appendectomy rates between patients with US findings suggestive of appendicitis and those with a clinical diagnosis. In contrast to previous studies, inconclusive US findings were also assessed, and similarly showed no significant difference in negative appendectomy rates when compared to clinical diagnosis. These results suggest that US does not improve diagnostic accuracy compared to clinical assessment. Ultimately, this may promote more judicious use of diagnostic imaging in the future.

Keywords: appendectomy, ultrasound, appendicitis

\section{MP56}

Redundant imaging of mandibular fractures in the ED: should we start with CT?

B. Peddle, MD, M. Klingel, MSc, S.L. McLeod, MSc, C. Richardson, $\mathrm{MD}$, University of Western Ontario, London, ON 
Introduction: Emergency department (ED) diagnosis of mandibular fracture is confirmed radiographically with either plain film X-ray or computed tomography (CT) scan. There is evidence that CT scans are more sensitive at detecting mandible fractures and that they are useful for pre-operative planning. The objective of this study was to assess imaging patterns for suspected mandible fracture and to determine if there are clinical criteria present which increase the likelihood of having a fracture. Methods: This was a retrospective medical record review of adult ( $>17$ years) patients who underwent an X-ray and/or CT scan for suspected mandibular fracture in one of two tertiary care EDs over a one year study period (Jan-Dec 2012). Patients with extensive facial trauma and suspected multiple injuries were excluded. A random sample of patients was examined for demographics, mode of imaging, clinical features on presentation, and diagnosis. Backwards stepwise multivariable logistic regression models determined predictor variables independently associated with having confirmed mandibular fracture. Results: 92 patients were included in the analysis. Mean (SD) age was $32.2(13.7)$ years and $70(75.3 \%)$ were male. $31(33.7 \%)$ patients were found to have a mandibular fracture and $61(66.3 \%)$ were fracture negative. Of those who were fracture positive, $13(41.9 \%)$ received an X-ray alone, 3 (9.7\%) had CT alone, and 15 (48.4\%) had both X-ray and CT scan. Of the patients that were confirmed fracture negative, $42(68.9 \%)$ received an X-ray alone, $13(21.3 \%)$ had CT alone, and $6(9.8 \%)$ had both an X-ray and CT scan. Presenting with malocclusion (OR: 6.1, 95\% CI: 1.6, 23.6) and presenting with gingival laceration congruent with the fracture location (OR: 24.3, 95\% CI: 2.7-215.9) were independently associated with having a mandibular fracture. Conclusion: A large proportion of patients with mandible fracture have both an X-ray and CT scan for diagnosis and/or prior to surgical management. Two clinical findings were independently associated with mandible fractures and may be useful in guiding emergency physicians towards CT initially, avoiding extra costs and unnecessary patient exposure to ionizing radiation.

Keywords: mandibular fractures, imaging

\section{MP57}

Criteria for transfer to tertiary trauma centers for traumatic brain injuries: validation phase based on the comparison of the evolution of patients transferred and non-transferred

N. Le Sage, MD, MSc, A. Lavoie, PhD, L. Moore, PhD, R. Verreault, $\mathrm{PhD}$, M. Emond, MD, MSc, Université Laval, Québec, QC

Introduction: Récemment, un consensus d'experts pluridisciplinaires a proposé une liste de critères de transfert vers les centres tertiaires de traumatologie. Il a été démontré que ces critères sont associés à une augmentation du risque de décès, d'admission à l'unité des soins intensifs et de complications sérieuses dans les centres tertiaires. La présente étude a pour but d'estimer le bénéfice du transfert chez la population des traumatisés cranio-cérébraux (TCC) reçus initialement en centre primaire ou secondaire et répondant à ces critères. Methods: Nous avons effectué les analyses sur une cohorte de patients initialement transportés dans des centres primaires ou secondaires entre 1998 et 2008. Pour chacun des critères, nous avons estimé le taux de décès des patients transférés ( $\leq 12$ heures), comparativement à ceux qui ne l'étaient pas. Les données ont été ajustées pour l'âge et la sévérité des blessures (NISS). Results: Sur une cohorte de 13135 patients, 1131 présentaient au moins un critères Delphi. Les patients présentant les critères suivants suite à un TCC ont un risque de décéder [IC $95 \%$ ] supérieur s'ils ne sont pas transférés en centre tertiaire : 1) score $<9$ sur l'Échelle de Coma de Glasgow (GCS), $\mathrm{RC}=0.18$ [0.06-0.52]; 2) fracture ouverte ou enfoncée du crâne, $\mathrm{RC}=0.21$ [0.06-0.71]; 3) altération de l'état de conscience avec tomodensitométrie anormale, $\mathrm{RC}=$ 0.35 [0.15-0.85] et 4) hématome sous-dural, épidural ou hémorragie intracérébrale, $\mathrm{RC}=0.53$ [0.36-0.79]. Conclusion: Les patients pré- sentant l'un ou l'autre des critères cités précédemment devraient systématiquement être transférés dans un centre tertiaire de traumatologie afin de diminuer leur risque de décéder.

Keywords: transfer, trauma care organization, major trauma

\section{MP58}

Does mode of transport confer a mortality benefit to trauma patients? Characteristics and outcomes at a single Ontario lead trauma hospital

I.M. Buchanan, MD, N. Sne, MD, McMaster University, Hamilton, $\mathrm{ON}$

Introduction: Rapid delivery of trauma patients to centres of definitive care was advanced with the use of rotor-wing aircraft in the Vietnam war. While consensus about the use of helicopter transport in some instances has emerged, it is unlikely that its use improves outcomes in all circumstances; few evidence-based guidelines exist to triage one form of transport over another. This study serves to establish an evidence-base for mode of trauma transport to a single Ontario Level I Trauma Centre. Methods: This is a retrospective, observational study. Data will be drawn from a chart-abstracted registry from the years 1995-2013. Inclusion criteria: age $>18$ years, Trauma Team activation, ISS $>12$, and transport direct from scene. Exclusion criteria: death prior to transport, missing data for TRISS-L statistic, $>30 \%$ missing data for descriptive statistics, and mixed transport modes. Rotor-wing versus ground transported cohorts are compared. Abstracted data includes demographic, injury severity, temporal and mortality variables. Statistical methods include D'Agostino \& Pearson normality test, median/IQR as measures of central tendency, MannWhitney/Fischer's Exact tests. TRISS-L analysis is performed to examine implied mortality benefit, by comparing actual mortality with projected mortality based on logistic regression algorithm validated for the population studied. Results: 3146 records met inclusion/exclusion criteria, with 387 rotor-wing and 2759 ground patients. Rotor-wing patients were younger ( 39 vs. 47 yr.), with a higher Injury Severity Score ( 25 vs. 22$)$, and a higher overall unadjusted mortality (16 vs. $9 \%)$ than ground patients $(p<0.001)$. Total prehospital time was longer for rotor-wing patients (73 vs. $45 \mathrm{~min}$.). Ground patients under-performed their predicted mortality, with 1.4 more deaths/100 transports, whereas rotor-wing patients outperformed predictions with 5.2 fewer deaths $/ 100$ transports (Z-scores of 2.6 and -3.7 respectively). Conclusion: Rotor-wing and ground transported trauma patients represent heterogenous populations. Rotor-wing patients outperform their predicted mortality, implying a survival benefit conferred by the overall transport "package". Further research examining details of transport is necessary to draw any further conclusion about causality, and to establish bestpractice for triage of trauma patients to any given mode of transport. Keywords: TRISS, trauma, transport

\section{MP59}

Non-indicated telemetry for COPD patients might clog the ER P. Beaudry, MD, V. Huard, MD, J. Chauny, MD, MSc, J. Paquet, PhD, R. Daoust, MD, M. Vincent, MD, M. Roy, MD, Université de Montréal, Montréal, QC

Introduction: To determine if the prescription of inadequate cardiac monitoring for chronic obstructive pulmonary disorder (COPD) patients is associated with longer length of stays in the emergency room (ER). Methods: Using our administrative database, we have retrospectively selected every consecutive COPD patient that was treated in our ER. $(n=4117)$. We excluded the patients with potential hemodynamic instability or with concomitant diagnosis that could have justified the prescription of telemetry. We analyzed the length of stays $(\mathrm{n}=1849)$ in regard to patients discharged home $(\mathrm{n}=615)$ or admitted 
$(\mathrm{n}=1234)$. Results: 105 (17.1\%) of COPD patients who were discharged have had a cardiac monitor compared to $283(22,9 \%)$ of the admitted ones. The non-admitted patients stayed 8.0 hours longer $(21.0$ VS 13.0) when a telemetry was prescribed. The admitted ones with cardiac monitoring had a length of stay of 6.7 hours longer (26.1 VS 19.4) compared to those who did not have any telemetry. Also, when controlled for age, sex and type of arrival (ambulance or on foot) with a linear multivariate regression analysis, we found that the length of stay was 10.1 hours longer on average (IC $95 \%: 7.0$ to 13.1). Conclusion: For patients with COPD diagnosis, non-indicated telemetry allocation was associated with significant prolongation of length of stay and might contribute to ER overcrowding.

Keywords: telemetry, monitoring, COPD

\section{MP60}

Variability of CTAS scoring in two tertiary care centres in Calgary A. Jervis, MD, S. Jones, MD, MHSc, D. Wang, MSc, E. Lang, MD

University of Calgary, Calgary, AB

Introduction: The Canadian Triage and Acuity Scale (CTAS) is a tool, utilized by triage nurses in all Emergency Departments (EDs) throughout Canada, which allows a team of health care providers to more accurately determine the order and priority of each patient's treatment. Average CTAS scores are also considered when administration determines ED staffing and funding. In 2011, it was identified that all Calgary area EDs have approximately the same average CTAS score. We would expect that Foothills Medical Centre (FMC), our regional trauma, stroke and coronary intervention centre, would have a lower average CTAS than one of our community hospitals, the Peter Lougheed Centre (PLC).The objective of this study is to investigate variability in CTAS score utilization by validating them against other measures of acuity at two sites. Methods: We conducted a retrospective cohort study in Calgary, Alberta. Data was drawn from electronic health record-derived sources from FMC and PLC. Inclusion criteria included presentation to the ED at either FMC or PLC in 2012. Twotailed t-test was used to test significant difference. Results: In 2012, FMC and PLC saw 80,313 and 80,744 patients in their EDs, respectively. The average CTAS score was lower for FMC than for PLC ( 2.83 vs. $3.03, \mathrm{P}<0.01)$. An increased admission rate was seen at FMC ( $24.7 \%$ vs. $14.9 \%)$ for CTAS level 1 ( $75.4 \%$ vs. $53.9 \%)$, 2(38.4\% vs. $28.1 \%$ ), and $3(20.3 \%$ vs. $14.5 \%)$, with no difference between FMC and PLC admission rates at CTAS level 4 or 5 . A higher proportion of patients at FMC presented with stroke $(2.1 \%$ vs. $0.4 \%)$, myocardial infarction $(0.7 \%$ vs. $0.4 \%)$ or required blood transfusion $(2.6 \%$ vs. $1.4 \%)$. FMC patients were more likely to arrive via $\operatorname{EMS}(31.2 \%$ vs. $17.4 \%)$, and had a higher 30 -day mortality rate $(2.0 \%$ vs. $0.9 \%)$ than PLC. Conclusion: Assuming similar thresholds to hospitalization, we have shown differential application of CTAS between two sites in Calgary. CTAS score alone may not appropriately depict differences in acuity between hospitals, and should not be the sole driver of administrative decisions such as staffing and funding. Efforts to standardize the application of CTAS are warranted.

Keywords: emergency medicine, CTAS, triage

\section{MP61}

Management and outcomes of superficial thrombophlebitis diagnosed in the emergency department

C. Lang, S. Chen, D. Ghag, Pharm D., E. Grafstein, MD, D.R. Harris, MD, MHSc, Faculty of Pharmaceutical Sciences, UBC, Vancouver, BC

Introduction: $10 \%$ of superficial thrombophlebitis (ST) patients progress to venous thromboembolism (VTE). Our objective was to describe a cohort of patients with ST within two large emergency departments, outline rates of anticoagulation, and determine outcomes. The primary outcome was the rate of death, major bleeding, or rehospitalization at any regional emergency department (ED) between anticoagulated and untreated ST patients, within 9 months after ED diagnosis. Methods: This was a retrospective medical records review study. Patients were identified from the St. Paul's Hospital and Mt. St. Joseph's Hospital Emergency Department Discharge Database. St. Paul's is a large academic inner city hospital with an ED census of 78,000 visits; Mt. St. Joseph's is an acute care community hospital with a census of 25,000 . Patients were identified from a larger VTE cohort. Included patients were those diagnosed with isolated ST. Excluded patients were those with incomplete data, no ultrasound confirmed diagnosis, those admitted to hospital, with active cancer, pregnant, or concomitant DVT / PE at time of diagnosis. Charts were reviewed from the period Jan. 1, 2008 to Dec. 31, 2012. Data collected was patient demographics, clot morphology, management within the ED (including anticoagulation), and outcomes. Outcomes were determined from linkage to a Regional Database (recurrence, readmissions, major bleeding) and Vital Statistics, with a follow-up period of 9 months. Providence Health Care REB granted ethical approval. Results: In the study period, 1196 patients were identified with VTE. Of those, 85 had ST; 35 were excluded due to concomitant PE / DVT, leaving 50 patients in the cohort. The average age was 57 years; $58 \%$ were female. $68 \%$ were lower extremity ST; $8 \%$ were upper extremity; $24 \%$ were unspecified (at the time of abstract submission). 27 patients $(54 \%)$ were anticoagulated to a therapeutic level; 23 were not $(46 \%)$. The majority of those anticoagulated were treated with a combination of dalteparin and warfarin $(20 / 27,74 \%)$; other treatment options included a combination of other low-molecular weight heparins and coumadin; low-molecular weight heparin only; NSAIDs; or no treatment. There were no events (readmissions or major bleeds), or deaths in either treatment group within 9 months of initial ED diagnosis. Conclusion: Within this small series of patients with isolated ST, treatment was highly variable. There were no readmissions, major bleeds or deaths in this cohort. Opportunities exist for study of the optimal management of patients with ST - the role of anticoagulation, and an ideal anticoagulation regimen for ST needs to be defined. Currently, the only randomized trial evidence supports the use of fondaparinux for treatment of ST; fondaparinux is not widely available and is more expensive than standard therapies.

Keywords: superficial thrombophlebitis, venous thromboembolism, treatment

\section{MP62}

Emergency department management of pulmonary embolism: a significant opportunity for standardizing care

D. Ji, H. Chen, D. Ghag, Pharm D., E. Grafstein, MD, D.R. Harris, MD, MHSc, Faculty of Pharmaceutical Sciences, UBC, Vancouver, BC

Introduction: Pulmonary embolism (PE) is a frequently managed condition in the emergency department (ED). Often, patients can be discharged with outpatient anticoagulation therapy. Risk scores exist, such as the Pulmonary Embolism Severity Index (PESI), to assist physicians in safe disposition (PESI $<85$ supports outpatient therapy). Our objective was to describe a cohort of patients with PE within two large emergency departments, outline ED management, identify rates of admission, and determine outcomes. Our primary objective was to determine whether PE patients were managed according to current published guidelines and determine outcomes. Methods: This was a retrospective medical records review study. Patients were identified from the St. Paul's Hospital and Mt. St. Joseph's Hospital Emergency Department Discharge Database. St. Paul's is a large academic inner city hospital with an ED census of 78,000 visits; Mt. St. Joseph's is an 
acute care community hospital with a census of 25,000 . Patients were identified from a larger VTE cohort. Included patients were those diagnosed with PE in the ED. Excluded patients were those with incomplete data, no imaging-confirmed diagnosis, those with renal dysfunction, with active cancer, pregnant, or critically ill. Charts were reviewed from the period Jan. 1, 2008 to Dec. 31, 2012. Data was collected on patient demographics, location of PE, management within the ED (including admission to acute care), and outcomes. Outcomes were determined from linkage to a Regional Database (recurrence, readmissions, major bleeding) and Vital Statistics, with a follow-up period of 9 months. Providence Health Care REB granted ethical approval. Results: For the study period, 1293 VTE patients were identified; from that, $77 \mathrm{PE}$ patients were identified for inclusion in this study. 59\% were male; average age was 51 . Of those, 39 / 77 (51\%) patients were managed as outpatients, $38 / 77$ (49\%) were admitted to hospital. The outpatient group had a mean PESI score of $62.8(\mathrm{SD}=20.6)$, whereas those admitted had an average score of $75.2(\mathrm{SD}=25.2)(\mathrm{t}$-test $=2.4$, $\mathrm{p}=0.02,95 \% \mathrm{CI}=2.02,22.8)$. Of those admitted to hospital, $22 / 38$ $(57.9 \%)$ had PESI scores < 85 ("inappropriately admitted"); whereas, 4 / 39 (10.3\%) discharged had PESI scores $>85$ ("inappropriately discharged"). From this cohort, ED physicians made appropriate decisions on admission or discharge at an accuracy of $66.2 \%$. Only 1 patient died due to non-VTE causes (in the inpatient cohort); no VTE or hemorrhage-related mortality was observed in either cohort. Four patients (4 / $39,10.2 \%)$ in the outpatient cohort had recurrent VTE events, versus one in the inpatient cohort $(1 / 38,2.6 \%)$ : all had non-therapeutic INR's at time of event (Fisher test $\mathrm{p}=0.35$ ). Conclusion: In this large cohort of PE patients diagnosed and managed in the ED, there is opportunity for improvement through standardization of care. Many patients in this cohort could have been safely managed as outpatients, saving significant costs. However, appropriate therapeutic anticoagulation needs to be maintained through careful monitoring. The role of new oral anticoagulants may be beneficial in this patient population. Keywords: pulmonary embolism, treatment

MP63

Diagnosis of chest pain by metabolic fingerprinting of serum T. Jelic, MD, E. Weldon, MD, A. Shaw, Ph.D, R.A. Ducas, MD, J. Ducas, MD, University of Manitoba, Winnipeg, MB

Introduction: Chest pain is one of the most common entrance complaints in the emergency department (ED). Rapid diagnostic times involving all aspects of acute coronary syndrome (ACS) care is a major therapeutic goal. Chest pain secondary to ACS is hypothesized to promote a transformation in blood serum composition (e.g. markers of plaque rupture, inflammation, ischemia) that is reflected as characteristic changes ("fingerprints") in the corresponding spectra of serum samples. The objective of this prospective cohort study is to discover and exploit these characteristic changes, and thus to develop a novel, rapid and sensitive diagnostic test to rule in/rule out ACS as the origin of chest pain in ED patients for whom EKG and traditional infarction markers are negative. Methods: Serum samples $(\mathrm{N}=75)$ were provided by patients presenting with chest pain at the St. Boniface General Hospital ED in Winnipeg, Manitoba, Canada. The ultimate diagnosis (ACS and non-ACS) was determined through chart review. The sera were characterized using a spectroscopic method (infrared (IR) spectroscopy) that provided metabolic "fingerprints"; samples were obtained via microfluidic preprocessing of serum (to recover metabolites and other non protein components) and IR spectra acquired. The spectra for ACS and non-ACS patients were compared.. Firstly, class averages were simply compared. Secondly, a multivariate analytical technique was used; a feature selection algorithm (GA_ORS; "genetic algorithm/optimal region selection"), with linear discriminant analysis, was adopted to identify IR spectral sub-regions optimal to identify ACS. The "classifier", trained using $2 / 3$ of the subjects ( 51 subjects; "training set"), was validated through its ability to correctly predict diagnosis for the remaining 1/3 subjects (24 subjects; "test set"). Results: The IR spectroscopic fingerprints considered here for (protein-depleted) serum represent the metabolic fingerprints hypothesized to carry information diagnostic of chest pain. The class average spectrum for a preliminary subset of 34 ACS patients was significantly different from that for a corresponding set of 41 control (non-ACS) patients. The multivariate analytical approach yielded a classifier that is $80 \%$ accurate $(79 \%$ sensitivity, $83 \%$ specificity) in distinguishing ACS from non-ACS patients. Conclusion: These results suggest that metabolic fingerprinting measurements have the potential to rule in or out ACS. Ongoing research will incorporate additional spectroscopic measurements (magnetic resonance spectroscopy, and mass spectrometry fingerprinting) and include larger numbers of diverse patient groups, with the aim of providing accurate tests for routine use to triage ED chest pain patients, and potentially developing a tool for risk stratification.

Keywords: cardiac biomarkers

\section{MP64}

The utility of chest $x$-ray as part of the investigation of patients with chest pain suspected to be cardiac in origin

S.G. Campbell, MB BCh, I. Cajee, MBBCh, S. Field, MB BCH, K. Magee, MD, M. Butler, BSc, C.L. Campbell, MB BS, Dalhousie University, Halifax, NS

Introduction: Chest x-rays (CXR) are often ordered in ED patients with chest pain as a matter of 'routine'. In patients with chest pain of cardiac origin, these are frequently normal, and routine use thereof may result in significant cost to the system with little benefit to patients. Our goal was to see if there was a subset of this group of patients who might safely forego CXR. Methods: The medical records of 984 patients who had been referred to cardiology for the investigation of chest pain were retrospectively reviewed. Clinical details and CXR results were reviewed independently by four experienced ED faculty (average 25,5 yrs experience, range 18-37). Reviewers indicated if they thought the CXR was helpful, or might contribute to ED care, or whether the result added nothing to care. A result was considered 'relevant' if any of the reviewers considered it so. Patients were excluded of they met any 9 of the 10 Rothrock CXR criteria (including temperature $>37.9 \mathrm{c}$ ) for ED CXR (we omitted age as an exclusion criteria). Results: Of 984 CXR results, 77 (7.8\%) were considered relevant by any one of the reviewers. 20 of these patients had temperatures 37.5 37.9. Patients with a temperature 37.5-37.9 of were 4.45 times more likely to be assessed as having a relevant CXR. Including only patients with a temperature $<37.5,5.8 \%$ of CXR were relevant. Although the chance of relevant CXR result increased with age group, however this did not reach statistical significance. Mean age in patients relevant CXR: 67.4 vs 60.9 years in those without a relevant result. No other variables were associated with CXR findings. Conclusion: Afebrile patients with chest pain suspected to be of cardiac origin rarely gained any benefit from CXR. We were not able to identify a subset of afebrile patients in whom CXR should be eliminated, but clearly more judicious use of CXR in this group of patients is indicated.

Keywords: chest $\mathrm{X}$-ray, investigations, chest pain

\section{MP65}

Point of care ultrasound in rural emergency departments: online survey examining access, training and utilization

P. Léger, MD, R. Fleet, MD, PhD, J. Maltais-Giguère, MSc, J. Plant, $\mathrm{MD}$, Piette, MD, F. Légaré, MD, PhD, J. Poitras, MD, Department of Family and Emergency Medicine - Laval University, Québec, QC 
Introduction: Point of care (POCUS) is an exam that can be rapidly performed at the patient's bedside with the purpose of answering specific and potentially life threatening clinical questions and improving safety of procedures. The Canadian Association of Emergency Physicians (CAEP) highlighted the importance of providing 24/7 access to POCUS in EDs nationwide. Despite CAEP's position on POCUS and its potential benefits, actual use of POCUS in Canada remains unclear. In the context of limited access to formal ulltrasound and CT scans in rural hospitals, POCUS has the potential to improve patient-care. We sought to examine access to POCUS and potential barriers/facilitators to its use among rural emergency physicians. Methods: This is a descriptive cross-sectional study using an online survey (survey monkey). The 30 - item questionnaire is an adapted and translated version of a previous survey conducted in rural Ontario by Flynn et al 2012. It contains open - ended, likert scale and yes/no type items. The questionnaire was pre-tested for clarity and relevance in a sample of emergency medicine residents with POCUS training $(\mathrm{N}=10)$. The survey was sent to regular staff physicians working either full or part time in rural EDs $(\mathrm{n}=206)$. The EDs were located in "rural and small towns" and provided 24/7 medical coverage with acute care hospitalization beds and were part of a larger province-wide rural ED study. We sent up to 4 email reminders over an 8 week period and contacted ED chairs in order to foster participation. Results: In total, 108 surveys were completed (participation rate $=52.4 \%$ ). Ninety three percent were family physicians , 7\% CCFP (EM) with a median seven years of practice experience. A bedside ultrasound device was available in $95 \%$ of rural EDs, $75.9 \%$ of physicians reported using POCUS on a regular basis. The most common indications for using POCUS were to rule out an abdominal aortic aneurysm $(70.4 \%)$ and to evaluate the presence of free fluid in trauma and intrauterine pregnancy $(60 \%)$. Limited access to training programs was the most common reason $(73 \%)$ for not using POCUS. More than $40 \%$ of POCUS users received training within their medical curriculum. Sixty four percent received training from the Canadian Emergency Ultrasound Society, $13 \%$ CAEP, 23\% other course. Finally, $95 \%$ of respondents stated POCUS skills are essential for rural ED practice. Conclusion: To the best of our knowledge, this is only the second study to examine POCUS use in rural EDs in Canada. Results suggest POCUS use is very good in rural EDs of Quebec. Yet, improved access to formal training is requested. Despite having the highest participation rate to date, response bias of enthusiastic POCUS users cannot be excluded. Nationwide studies on POCUS use are also required.

Keywords: imaging, ultrasound, point-of-care ultrasound

\section{MP66}

Pilot study assessing high-sensitivity cardiac troponin assays and change in concentrations for the prediction of hospital admission for acute coronary syndrome

C. Shortt, BSc, A. Worster, MD, S. Hill, PhD, P. Kavsak, PhD, McMaster University, Hamilton, ON

Introduction: Emerging evidence suggests that change in high-sensitivity cardiac troponin concentrations ( ) improves diagnostic performance. Two assays (Abbott ARCHITECT hsTnI, Roche Elecsys hsTnT) have Heath Canada approval. Guidelines recommend measurement 3-6h after presentation for change measurement, but do not specify if absolute $(\mathrm{ng} / \mathrm{L})$ or relative differences (\%) should be used. We compared the diagnostic performance of $\mathrm{ng} / \mathrm{L}$ vs. \% for both hsTn assays between $0-3 \mathrm{~h}, 3-6 \mathrm{~h}$, and $0-6 \mathrm{~h}$ in emergency department (ED) patients presenting early with potential cardiac-ischemic symptoms. Methods: We measured hsTnI and hsTnT levels in serum samples (storage $-80^{\circ} \mathrm{C}$ ) taken at $0,3,6 \mathrm{~h}$ from 85 patients presenting with potential cardiac ischemic symptoms that occurred within $6 \mathrm{~h}$ prior to arrival at the ED. We performed ROC curve analyses to com- pare the areas under the curve (AUC) for $\mathrm{ng} / \mathrm{L}$ vs. $\%$ at all 3 time intervals for both assays (DeLong method) with the Youden index (J) and likelihood ratios (LR) for the that was significant for predicting hospital admission for acute coronary syndrome (ACS=myocardial infarction or refractory ischemic cardiac pain) within $72 \mathrm{~h}$ after presentation. Results: The peak concentration (ng/L) was significantly higher in those admitted for ACS [median (IQR) hsTnI=918(4410466), hsTnT=123(33-588); n=10] vs. those who were not [hsTnI=6(4-12), hsTnT=6(<3-18); $\mathrm{n}=75)(\mathrm{p}<0.01)]$. Comparison between $\mathrm{ng} / \mathrm{L}$ vs. $\%$ at $0-3 \mathrm{~h}, 3-6 \mathrm{~h}$, and $0-6 \mathrm{~h}$, yielded significantly higher AUC for $\mathrm{ng} / \mathrm{L}$ for the hsTnT at all 3 time intervals $(\mathrm{p}<0.05)$, however for hsTnI the $\mathrm{ng} / \mathrm{L}$ was significantly higher only for the 0 $6 \mathrm{~h}$ interval $(\mathrm{p}=0.03)$. At the $0-6 \mathrm{~h}$ time interval the hsTnI $\mathrm{ng} / \mathrm{L}$ outperformed the hsTnT ng/L [AUC $(95 \% \mathrm{CI}) \mathrm{hsTnI}=0.91(0.83-0.96)$ vs. hsTnT $=0.75(0.64-0.83), \mathrm{p}=0.02]$. The optimal absolute change $(\mathrm{J})$ at $0-6 \mathrm{~h}$ time interval for the hsTnI test was $>24 \mathrm{ng} / \mathrm{L}$ with a positive $\mathrm{LR}=26(95 \% \mathrm{CI}: 6-109) /$ negative $\mathrm{LR}=0.3(95 \% \mathrm{CI}: 0.1-0.8)$ and for the hsTnT test was $>14 \mathrm{ng} / \mathrm{L}$ with a positive $\mathrm{LR}=15(95 \% \mathrm{CI}: 4-51) /$ negative $\mathrm{LR}=0.4(95 \% \mathrm{CI}: 0.2-0.9)$. Conclusion: These data suggest that absolute is superior to relative changes when using high-sensitivity cardiac troponin tests for predicting ACS hospital admission for patients presenting early after pain-onset. By $6 \mathrm{~h}$ a change in concentration equivalent to the manufacturers' reported 99th percentiles (hsTnI $=26$, hsTnT=14) was most optimal. These findings require prospective validation.

Keywords: emergency medicine, acute cardiac injury, high-sensitivity cardiac troponin

\section{MP67}

Paramedic recognition and management of anaphylaxis in the prehospital Setting

S.K. Sandhanwalia, Hons. BSc., MD, K. Samoraj, MD, M. Welsford, MD, McMaster University, Hamilton, ON

Introduction: Anaphylaxis is a life-threatening condition that paramedics frequently face in the field. Few published reports have evaluated paramedic management of anaphylaxis in Canada. Most cases of anaphylaxis are out of hospital, and delay in epinephrine administration increases mortality. Immediate paramedic recognition and response is paramount. The primary objective of this study was to determine the proportion of cases that met the definition of anaphylaxis and were administered epinephrine by paramedics. The secondary objective was to determine the proportion of anaphylaxis cases administered epinephrine within 10 minutes of patient contact. Methods: This was a retrospective observational study of patients with anaphylaxis managed by primary or advanced care paramedics in 6 Emergency Medical Service Areas in Ontario, from January 1, 2012 to December 31, 2012. All ambulance call records (ACR) coded as local allergic reaction (code 84) or anaphylaxis (code 85) were reviewed by the authors, to determine if the patients met the definition for anaphylaxis as outlined by International Guidelines. The timing of all medications administered and procedures completed were abstracted into a database. Descriptive data is reported using proportions and medians with confidence intervals (CI). Results: A total of 117 ACRs were reviewed. Of the cases coded as anaphylaxis 55/65 (85\%), were correctly identified. However $27 / 50(54 \%)$ of cases were incorrectly coded as local allergic reaction when they met anaphylaxis criteria. $35 / 82(43 \%)$ of cases meeting anaphylaxis criteria received a treatment other than epinephrine. Epinephrine was administered in 47/82 (57\%) of anaphylaxis cases. 47/55 (86\%) received epinephrine when paramedics correctly recognized anaphylaxis. $36 / 47$ (77\%) of these received epinephrine in 10 minutes or less, with a mean of 7.6 minutes (95\% CI: 6.5-9.0). Conclusion: There appear to be gaps in the recognition of anaphylaxis by paramedics in Ontario. Similarly, gaps exist in 
the life-saving use of epinephrine for anaphylaxis, since it was administered in just over half of cases. However, when epinephrine was administered it was done so in a timely manner for most patients. As part of a quality improvement initiative, this data will be used to evaluate and enhance the training and medical directives utilized by paramedics to improve their recognition and management of patients with anaphylaxis.

Keywords: anaphylaxis, epinephrine, paramedics

\section{MP68}

Alternatives to traditional EMS dispatch or transport to ED: a scoping review of published outcomes

J.L. Jensen, BSc, A. Carter, MD, J. Rose, S. Visintini, MLIS, J. McVey, MD, E. Bourdon, MSc, R. Brown, BSc, A. Travers, MD, MSc Dalhousie University, Halifax, NS

Introduction: EMS programs which provide an alternative to traditional EMS dispatch or transport to the emergency department (ED) are becoming widely implemented. The objective was to catalog all outcomes used to measure such alternative EMS programs. Methods: Systematized bibliographic searches were conducted in PubMed, Embase, CINAHL and the Cochrane Library. The grey literature search included a list of websites developed by the study team. Searches were purposefully broad. Inclusion criteria were: 911 callers/EMS patients, reports on alternatives to traditional ambulance dispatch OR traditional ambulance transport to the ED, and reports an outcome measure. The reports were categorized as either alternative to dispatch or to transport, and outcomes were categorized and described. Results: The bibliographic search retrieved 8773 titles, from which 134 titles were selected for further review. From these, 61 abstracts were retrieved, out of which 27 full text articles were included, with an additional 15 added from reference list hand-searching $(\mathrm{n}=42$ included). In the grey literature search, 31 websites were identified, from which four met criteria and were retrieved. Fourteen reports described alternatives to EMS dispatch, measuring the following outcomes: clinical ( $\mathrm{n}=1$ outcome, 1 article), safety ( $\mathrm{n}=4$ outcomes, 4 articles), time ( $\mathrm{n}=2$ outcomes, 2 articles), service utilization ( $\mathrm{n}=5$ outcomes, 7 articles), patient satisfaction $(\mathrm{n}=$ 1 outcome, 2 articles), cost ( $n=3$ outcomes, 3 articles), accuracy of decision ( $\mathrm{n}=4$ outcomes, 6 articles), and process outcomes ( $\mathrm{n}=1$ outcome, 1 article). Thirty-one reports described alternatives to EMS transport, measuring the following outcomes: clinical $(\mathrm{n}=7$ outcomes, 10 articles), safety ( $\mathrm{n}=5$ outcomes, 13 articles), time ( $\mathrm{n}=7$ outcomes, 7 articles), service utilization ( $\mathrm{n}=8$ outcomes, 13 articles), patient satisfaction ( $\mathrm{n}=2$ outcomes, 11 articles), cost ( $\mathrm{n}=4$ outcomes, 5 articles), accuracy of decision ( $\mathrm{n}=5$ outcomes, 9 articles), process outcomes ( $\mathrm{n}=$ 7 outcomes, 7 articles), other ( $\mathrm{n}=1$ outcome, 2 articles). Conclusion: A large variety of outcomes used to report on alternative EMS programs were cataloged and described. Researchers and program leaders should achieve consensus on uniform measures, to allow bench-marking and comparison across programs

Keywords: emergency medical services, dispatch, alternatives

MP69

Pre-hospital management of patients with history of asthma and COPD in one Canadian urban setting

T. Tamura, T. Chung, A. Kabaroff, MD, K. Liss, BSc, C. Villa-Roel, B.H. Rowe, MD, MSc, University of Toronto, Toronto, ON

Introduction: Asthma and chronic obstructive pulmonary disease [COPD] exacerbations present similarly with dyspnea, cough and wheezing; however, other factors may assist emergency medical services (EMS) staff differentiate diseases and guide management. This study characterized EMS patients $<55$ presenting with acute respiratory distress and compared demographics and initial management based on their asthma or COPD history. Methods: Electronic patient care records (PCRs) from the Edmonton Zone EMS in 2012 were retrospectively reviewed to identify calls made by adults for acute respiratory distress and record treatments. Data were collected by trained staff using the REDCap data platform. Descriptive data are reported using proportions and medians with interquartile range (IQR). Results: From 457 PCRs, 197 (43\%) reported asthma history and 77 $(17 \%)$ reported CODP history. Both groups had mostly female patients (58\% vs. $70 \%)$ with a slightly older population in the COPD group. Self-medication was recorded in $88(45 \%)$ of the asthmatics and $29(38 \%)$ of the COPD patients. Paramedics administered short acting beta-agonists ( $98 \%$ vs. $99 \%)$, short acting anticholinergics $(77 \%$ vs. $87 \%)$, oral corticosteroids $(33 \%$ vs. $36 \%)$ and magnesium sulphate (14\% in both groups) similarly to both groups. More patients with history of COPD required oxygen therapy ( $68 \%$ vs. $75 \%$ ), cardiac monitoring ( $55 \%$ vs. $82 \%$ ), and were transported to the hospital (85\% vs 95\%) after EMS treatment. Conclusion: Asthma and COPD patients receive virtually identical treatments in the EMS environment; however, COPD patients are more severe and require more airway interventions. Improved diagnostic differentiation may improve personalized evidence-based treatments.

Keywords: emergency medical services, COPD

\section{MP70}

The association of adverse events during procedural sedation with airway assessment

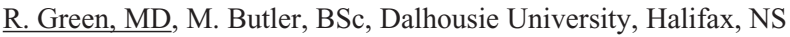

Introduction: Airway management is of primary importance in procedural sedation (PSA) in the Halifax Infirmary (HI) emergency department (ED). Traditionally, an airway assessment assesses difficulty of intubation by paramedics. It is unknown whether this assessment is predictive for adverse events (AEs) during PSA. We report whether features of airway assessment for PSA are associated with the occurrence of AEs. Methods: This is a retrospective analysis of 4330 PSAs from the PSA registry, and comparison of a number of common airway assessments with AEs. Our outcomes are as follows: - Hypotension Hypoxia - Tachycardia - Bradycardia - Tachypnea - Bradypnea Our primary outcome was the occurrence of ANY of the measured AEs. We report the prevalence of the above outcomes of the population. To assess for association between airway assessment and AEs, a logistic regression model controlled for confounding effects such as age and gender. Results: Neck pathology (3.7\%) and snoring (29.9\%) were associated with an increased risk of an AE, while decreased risk was found in male patients. Mouth opening (82.9\%) and being male $(48.8 \%)$ were associated with a reduced incidence of AEs. Hypotension was more likely with snoring, and less likely in males. Tachycardia is less likely with a worse malampatti score, and more likely in obese and male patients. Tachypnea is less likely with mouth opening wider than three fingers, and more likely with neck pathology and snoring. The airway assessment was not predictive for hypoxia. Conclusion: The presence of neck pathology and snoring are associated with a higher incidence of AEs during PSA, while mouth opening and being male was protective. More research into airway assessment may provide insight into the incidence of adverse events during PSA.

Keywords: airway management, procedural sedation

MP71

Referral patterns for pediatric emergencies: an assessment of knowledge needs for the Translating Emergency Knowledge for Kids (TREKK) Network

K. Moncrieff, PhD, MSc, C. Kruck, L. Knisley, Knowledge Broker, D. Goulard, D. Sinclair, MD, T. Klassen, MD, MSc, E. Lang, MD

University of Calgary, Calgary, $\mathrm{AB}$ 
Introduction: Referral and advice seeking practices for pediatric emergencies by non-specialized emergency physicians can provide important insight into unmet education needs. We examined referrals to Alberta Children's Hospital in Calgary and Stollery Children's Hospital in Edmonton from other sites in Alberta, including rural and remote sites, to determine what types of complaints were being referred and the results of the referrals. Our objective was to determine the frequency of different types of presenting complaint and referral dispositions overall, in each geographic zone, and in each age group. Methods: We categorized each referral by type of complaint, age group (0-2, 3-5, 6-12, 13-18, or over 18), geographic zone (Calgary, Edmonton, Central, North, South, or outside of Alberta), and referral disposition (advice provided over the phone, direct admission, referred to emergency department, referred to emergency department with specialist to see, referred to air, referred to outpatient clinic, or other). Results: There were 4066 referrals during the period of March 2012 to March 2013 covering 47 complaints. The most common presenting complaint ( $24 \%$ of total) was fracture, dislocation, or other musculoskeletal injury, and this was consistent across all zones. $44 \%$ of complaints in this category were managed with telephone advice only, $22 \%$ were referred to the emergency department, $13 \%$ were referred to the emergency department to see a specialist, $17 \%$ were referred to outpatient clinics, and 3\% were directly admitted. The next most common presenting complaints were respiratory problems (asthma, infection, and other) at $15 \%$ and abdominal pain at $6 \%$. Overall, $49 \%$ of referrals were referred to the emergency department, $29 \%$ were managed with advice by phone, $8 \%$ were referred to the emergency department to see a specialist, $7 \%$ were direct admissions, and $6 \%$ were referred to outpatient clinics. Conclusion: MSK injuries and respiratory infections are the most common problem for which emergency physicians seek pediatric specialist advice. These topics should be considered as high priority for knowledge mobilization and education.

Keywords: pediatrics

\section{Posters Presentations}

\section{P001}

Imaging in the Diagnosis of Acute Appendicitis in Children: A retrospective chart review

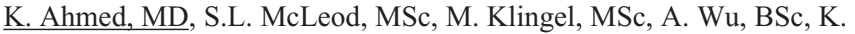
Theakston, MD

University Western Ontario, London, ON

Introduction: Among the diagnostic modalities available for appendicitis, ultrasound (US) and computed tomography (CT) are commonly used. The primary objective was to determine how many pediatric patients had imaging prior to appendectomy over the last 5 years, the type of imaging and results. A secondary objective was to determine the sonographic features of acute appendicitis associated with pathologically confirmed cases. Methods: This was a retrospective chart review of children $(<18$ years $)$ who underwent an appendectomy at a paediatric tertiary care centre during the last 5 years for suspected acute appendicitis. All children who underwent an appendectomy for other conditions were excluded. US results were categorized into 4 mutually exclusive groups: positive for appendicitis, indeterminate suggestive (I-S), indeterminate non-suggestive (I-NS) and normal. The diagnosis of appendicitis was determined by pathological examination. Results: 663 patients had an appendectomy. Of those, 400 were reviewed and 373 met the inclusion criteria. $75(20.1 \%)$ patients did not have any imaging prior to appendectomy. Of the 298 (79.9\%) patients who had imaging, 289 $(97.0 \%)$ had US, $7(2.3 \%)$ had US and CT and $2(0.7 \%)$ had CT alone. $225 / 296$ (76.0\%) US results were classified as positive, 207 (92\%) had appendicitis. 38 (12.8\%) US results were classified as I-S, $30(79.0 \%)$ had appendicitis. $32(10.8 \%)$ US results were classified as I-NS, 22 $(68.8 \%)$ had appendicitis. $1(0.4 \%)$ US report was normal and did not have appendicitis. Of the 296 patients who had an US prior to surgery, $35(11.8 \%)$ were found to have a normal appendix. Of these, $17 \mathrm{had}$ a positive US report $(\mathrm{n}=225)$, resulting in a $7.5 \%$ false-positive rate. 17 patients had indeterminate US findings $(\mathrm{n}=70)$, resulting in a $24.3 \%$ false-positive rate. The mean (SD) appendiceal diameter was larger in the patients who had a positive US and appendicitis, compared to patients who had a positive US but a negative pathology $(10.4 \mathrm{~mm}$ vs. $7.4 \mathrm{~mm}$; 3.0; 95\% CI: 1.7, 4.3). Conclusion: Of the 373 patients who had an appendectomy for suspected appendicitis, close to $80 \%$ had imaging prior to surgery. The use of CT remains limited. While a positive US is a reliable diagnostic test for appendicitis with a low false-positive rate, indeterminate US findings are less predictive. The overall negative surgery rate for pediatric appendicitis is $11.5 \%$.

Keywords: appendicitis, paediatrics, imaging

\section{$\mathrm{P} 002$}

Acute immobilization of Colles fractures in the ED - splinting vs. circumferential cast

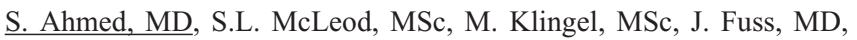
University Western Ontario, London, ON

Introduction: Although distal radius fractures ("Colles" fractures) are commonly treated in the emergency department (ED), physician opinions and practices differ with regards to acute immobilization strategies. Evidence is sparse and guidelines remain vague regarding best practice for immobilization. The objective of this study was to determine current ED practices for acute immobilization (splinting versus circumferential casting) of Colles fractures and to investigate unplanned return visits to the ED for cast complications and/or worsening of initial injury. Methods: This was a retrospective medical record review of adult patients arriving to one of two EDs at a tertiary care centre (annual census $125,000)$ with distal radius fractures that required reduction during initial visit over a one year period (April 2011-March 2012). Open fractures, fractures requiring surgery or ED orthopedic consultation, patients with neurovascular compromise and those with multiple nonulna/radius fractures were excluded. Patient demographics, initial immobilization technique, and information from follow-up and return visits within 14 days were recorded. Worsening of initial injury was defined as further displacement, angulation and/or impaction, or the need for surgical intervention. Results: 244 charts were reviewed, 117 were included in the analysis. Mean (SD) age was 60.4 (20.0) years. $106(90.6 \%)$ patients were circumferentially casted and $11(9.4 \%)$ were splinted. $100 \%$ of the splinted patients and $86(81 \%)$ of the casted patients had angulation, displacement or impaction at initial injury. Worsening of initial injury was found in $49(46.2 \%)$ casted patients and $6(54.5 \%)$ splinted patients at outpatient follow-up. $23(19.7 \%)$ patients had an unplanned return ED visit within 14 days relating to immobilization complications, all of which were circumferentially casted patients. Reasons for the return ED visits included pain, swelling and/or tightness and $13(56.5 \%)$ patients underwent bi-valving of their casts. Conclusion: Considering the complications associated with circumferential casting, ED physicians should consider splinting methods in acute Colles fractures. Future studies should assess the efficacy of splinting versus circumferential casting for this indication.

Keywords: radius fracture, immobilzation

P003

Bridging the pain gap between emergency department discharge and community care: a pilot evaluation of the Calgary 'Opioids For Home' program 
R.A. Allen, BEng, J.K. Tay, BSc, E.J. Saude, MD, PhD, T. Junghans, BA(Hons), BCC, E. Lang, MD, University of Alberta, Edmonton, AB

Introduction: Some Emergency Departments (EDs) dispense opioids directly to patients at discharge. Opioids For Home (OFH) programs provide short-term pain relief to bridge the gap between discharge and community care. In 2012, 11,124 OFH packs were dispensed in the Calgary Zone. The purpose of this multicentre pilot study was to evaluate the effectiveness of the OFH program based on patients' and physicians' experiences. Methods: We recruited patients who presented to the ED with acute pain and who received OFH consisting of 6 or 12 tablets of oral opioids; mainly oxycodone/acetaminophen. Patients were surveyed to assess perceived adequacy of pain management and overall program satisfaction with a 26 -item, pre-piloted instrument at discharge, day two, and day six post-discharge. Corresponding ED physicians completed a 6-item piloted survey to assess their perceptions of patient experiences with OFH. Results: Of 41 enrolled, 27 patients completed all three surveys. Of these, 21 (78\%) believed their injury required opioids for pain management; $10(37 \%)$ reported OFH as inadequate for pain management; and $25(93 \%)$ reported overall program satisfaction. 32 physicians completed 40 surveys. $36(90 \%)$ expected adequate pain management using OFH; 14 (35\%) expected that patients would require medical follow-up to manage pain; and 28 $(70 \%)$ expected follow-up physicians would provide additional analgesia. Results refer to participants who agreed or strongly agreed to the above statements. Conclusion: OFH appears to be beneficial and is appreciated by patients; however, there is a lack of agreement between physicians' expectations and patients' reported pain management. These results warrant further evaluation into OFH effectiveness to guide future program direction.

Keywords: pain management, analgesia, discharge instructions

P004

Diagnosis and management of children with anaphylaxis: a national survey of emergency physicians

W. Alqurashi, MD, I.G. Stiell, MD, MSc, G. Neto, MD, G. Wells, PhD Children's Hospital of Eastern Ontario, Ottawa, ON

Introduction: To assess the knowledge and attitudes of Emergency Physicians (EP) across Canada with respect to the diagnosis and management of children with anaphylactic reactions, and to identify factors associated with correct epinephrine administration. Methods: A crosssectional survey using simple random sampling. We used the membership database of the Canadian Association of Emergency Physicians (CAEP) as our sampling frame. Survey questions were based on two clinical scenarios involving an infant and an adolescent having an anaphylactic reaction after ingesting peanuts. Physicians were also queried on issues related to the choice of epinephrine concentration and route of administration in anaphylaxis, confidence level demonstrating to patients the correct use of epinephrine auto-injector (EAI), and utilization of written Anaphylaxis Action Plans (AAP) upon discharge from the Emergency Department (ED). The survey was administered using modified Dillman's technique. Primary outcome measures are correct diagnosis and treatment of the anaphylactic reactions. Results: Of the 615 EP surveyed, $340(55 \%)$ responded. The majority of respondents were male 214(63\%), practiced in academic settings with primarily an adult population $155(45 \%)$ or community hospitals $149(44 \%)$. Overall, $211(62 \%)$ of the physicians correctly agreed that both clinical scenarios were consistent with anaphylaxis, and 206(61\%) chose to administer epinephrine. Regarding knowledge about epinephrine administration, $280(82 \%)$ and $287(84 \%)$ identified correct epinephrine concentration and IM route of administration respectively. Only 148 (43\%) felt confident demonstrating the correct use of EAI to patients and families and only $155(45 \%)$ provided written AAP to patients upon discharge from the ED. In comparing pediatric EP and pediatricians to adult EP and general family practitioners, pediatric physicians were more likely to agree with the diagnosis of anaphylaxis for both scenarios ( $91 \%$ vs $64 \%, \mathrm{p}=0.02$ ), with treating both reactions with epinephrine ( $96 \%$ vs $64 \%, \mathrm{p}=0.002$ ), and be confident demonstrating to patients the correct use of EAI ( $82 \%$ vs $44 \%, p=0.01$ ). Conclusion: Although the majority of EP seem to have some knowledge of pediatric anaphylaxis, a substantial proportion have knowledge gaps that may negatively impact the quality of care provided to this vulnerable population. These gaps may be mitigated through further knowledge dissemination efforts.

Keywords: anaphylaxis, epinephrine, pediatric

P005

Can a simplified two view echocardiogram screen for severe aortic stenosis?

H. Alzahrani, MMBS, M.Y. Woo, MD, C. Johnson, MD, P. Pageau, $\mathrm{MD}, \mathrm{S}$. Millington, MD, V. Thiruganasambandamoorthy, MD, MSc, University of Ottawa, Department of Emergency Medicine, Ottawa, ON

Introduction: Aortic stenosis (AS) is a common valve problem that causes significant morbidity and mortality. Echocardiography has become the standard non-invasive means for evaluating the severity of AS. The goal of this study is to determine whether an emergency physician (EP) can screen for severe AS by reviewing only two B-mode echocardiographic views [parasternal long axis (PSLA) and parasternal short axis (PSSA)]. Methods: Health records and echo images were reviewed of a convenience sample of 60 patients with no AS, mild AS or severe AS between Jan 2011 and Dec 2012. All echocardiograms were performed by an expert echocardiographer and reviewed by a cardiologist. An EP blinded to the cardiologist's echocardiogram reports reviewed only PSLA and PSSA views after the cases were randomly sorted. A second EP independently reviewed $25 \%$ of randomly selected patients for inter-rater reliability. Severe AS was defined as no cusp movement seen by the EP reviewers. Collected data included patient demographics, data for each echo view (quality, the number of cusps visualized, presence and degree of calcification), and EP interpretation. The EP interpretation was compared to final cardiology reports. Analyses included descriptive statistics, test characteristics for severe AS, and kappa for agreement. Results: The mean age was 75.3 years (range 1890 ) with $36.7 \%$ female. The cardiologist's diagnosis was: $33.3 \%$ severe AS, $31.7 \%$ mild AS, and $35.0 \%$ no AS. The PSSA view was poorer in quality compared with the PSLA ( $\mathrm{p}=0.02$ ), but the PSSA view was better than PSLA to visualize all three cusps $(83.3 \%$ vs. $0 \%, \mathrm{p}=0.001)$. There was no difference in the presence of calcification between the mild and severe AS groups ( $\mathrm{p}=0.16$ ). The sensitivity and specificity for EP diagnosis of severe AS was 75\% (95\%CI 56.7-85.4\%) and $92.5 \%$ (95\%CI 83.3-97.7\%). The kappa for severe AS was 0.69 (95\%CI $0.41-$ 0.85 ) and there was no significant difference between observers in the quality of the view, presence and degree of aortic calcification and the number of cusps visible. There was poor correlation between the two observers in terms of which view was best to determine severe AS with a kappa of $0.10(0-0.50)$. Conclusion: Both the PSLA and PSSA views can be used by EPs to reliably interpret severe AS. High sensitivity which would be more desirable for screening, could possibly be achieved by defining severe AS as single cusp movement restriction. Future studies are required to address this issue.

Keywords: aortic stenosis, echocardiography

P006

Management of renal colic in patients presenting to regional emergency departments with and without a CT scanner N. Argintaru, BSc, N. Rafo, BSc, K. Milne, MD, D. Grushka, MD, M. Bhimani, MSc, MD 
University of Western Ontario - Schulich School of Medicine and Dentistry, London, ON

Introduction: Renal colic is a common Emergency Department (ED) presentation. The use of non-contrast Computer Tomography (CT) in diagnosing renal colic has increased and is the gold standard despite issues with costs, accessibility and potential radiation exposure. Ultrasonography (US) is a viable imaging modality but often limited to daytime use. This study evaluated how the presence of on-site CT scanners in community ED's affects the management renal colic. Methods: A retrospective chart review was conducted at five regional hospitals: three with a CT scanner, and two without. 200 adult ED renal colic patients were selected per site in reverse chronological order. Data extracted included demographics, past medical history, imaging (ED and outpatient), analgesia and follow-up. Results: Of 994 patient visits reviewed, $400(40.2 \%)$ were from sites without a CT and $594(59.8 \%)$ were from sites with a CT. $43.3 \%$ patients seen in an ED with a CT received a CT scan, versus $12.9 \%$ patients in sites without a CT $(\mathrm{p}<0.01)$. Accounting for outpatient CT referrals, $49.0 \%$ patients seen in an ED with a CT on site received a CT, as compared to $24.5 \%$. Patients who were seen in an ED with a CT were more likely to have an US ( $<<0.01)$, more likely to receive analgesia and more likely to be referred to outpatient urology. There was no difference in admission rates $(p=0.32)$. Conclusion: Overall, patients attending community hospitals without a CT scanner on site are less likely to receive a CT scan and also less likely to be imaged by any modality. An on-site CT scanner alters patient management in community hospitals by increasing the proportion of patients receiving analgesia, imaging and outpatient referral. The presence of CT scanners in community hospitals needs ongoing investigation to identify whether alterations in management of acute renal colic are clinically significant.

Keywords: imaging - computed tomography, renal colic, rural

\section{P007}

The impact of cmputed tomography (CT)-defined calculus-induced renal obstruction on the acute management and disposition of the renal colic patient

P. Massaro, MD, BScE, MASc, R. Pawsey, P.R. Atkinson, B. Archer, MD, T. Whelan, MD, Dalhousie University, St. John, NB

Introduction: The impact of CT-defined calculus-induced renal obstruction on patient presentation and its relevance to patient management is unclear. A retrospective cohort study was completed to determine whether the CT finding of obstruction predicted the outcome of non-surgical management and subsequent need for operative intervention. Methods: We reviewed consecutive charts of patients diagnosed with CT-confirmed, symptomatic, unilateral ureteric calculi in our Emergency Department (ED) over a two year period. Stones were categorized as obstructing or non-obstructing using standard CT features. The primary outcome was the 30-day ED re-presentation rate for renal colic after CT-diagnosis. The secondary outcome was operative intervention. Chi square tests were used for categorical variables, t-tests and Mann Whitney U-tests for continuous variables, and logistic regression identified associations with ED visits and operative intervention. Results: In total, 202 patients met inclusion criteria: 133 (65.8\%) had CT-defined obstruction, and 69 (34.2\%) did not. Patients with obstruction had significantly larger median stone size $(\mathrm{p}=0.001)$, and higher median creatinine $(\mathrm{p}=0.0369)$ than those without obstruction. Obstruction was not predictive of 30-day re-presentation after diagnosis $(\mathrm{OR}=0.67, \mathrm{CI} 95 \% 0.34-1.31, \mathrm{p}=0.246)$ or of operative intervention (OR 1.20, CI95\% 0.67-2.18, $\mathrm{p}=0.538$ ). On multivariate analysis, only stone size, number of renal colic ED visits before CT-diagnosis, and proximal stone location predicted surgical intervention. Conclusion: Although frequently reported on $\mathrm{CT}$, we demonstrate that the presence of unilateral renal obstruction does not predict the failure of medical management or the need for operative intervention. In the absence of absolute indicators for operative intervention, the CT-finding of obstruction should not alter the acute management plan of the renal colic patient.

Keywords: renal colic, clinical prediction, emergency medicine

\section{P008}

A large in-situ disaster simulation as a means for interdisciplinary and interprofessional whole hospital learning

I. Bank, MDCM, E. Khalil, MD, M. Ruddy, L. Varpio, PhD, M. Young, PhD, McGill University, Centre for Medical Education, Montréal, QC

Innovation concept: There has been increasing focus on learning in interprofessional and interdisciplinary environments. Many reported educational programs include members of different health professions, and many levels of learners. However, few have explicitly attempted to include the breadth of team members in a tertiary care centre. The complexities of multidisciplinary teams may be accentuated by stressful situations, such as mass casualty events. We report initial findings from a large multidisciplinary in-situ disaster simulation that occurred in a tertiary care pediatric hospital. Methods: An unannounced, largescale, city-wide, in-situ disaster simulation occurred in 2012. All hospital personnel were encouraged to participate, and were invited to complete a retrospective pre-post survey. The simulation focused on medical management, situational awareness, paradigm shift and crisis resource management (team training) in a mass casualty disaster. Curriculum, tool, or material: Participants included but not limited to: nurses, physicians, residents, respiratory technicians, social services, clerical staff, and others. 93 participants completed the survey, and reported that the simulation was valuable to their learning 5.7/6 $(6=$ strongly agree) and practice 5.7/6. Not all survey items were relevant to all participants, therefore medically (e.g. procedural skill related) and non-medically focused items (e.g. disaster plan related) were analyzed separately. Post simulation ratings were significantly higher for both medically focused items $(\mathrm{n}=53$; mean retrospective pre $=3.6 / 6(\mathrm{SD}=.2)$, post $=4.3(.2) ; \mathrm{F}(1,642)=44.1, \mathrm{p}<.0001)$, and non-medically focused items $(\mathrm{n}=83$; mean retrospective pre $=4.1 / 6(\mathrm{SD}=.2)$, post $=5.0(.2)$; $\mathrm{F}(1,228)=29.7, \mathrm{p}<.0001)$, and consistent across participant groups. Conclusion: Participants felt this simulation improved their ability to respond to a disaster involving children and that it was valuable to their learning. This experience supports the benefit of large-scale in- situ simulations as an opportunity for whole-team learning.

Keywords: innovations in EM education, disaster simulation, interprofessional and interdisciplinary

P009

Innovations in EM education - slit lamp use

D.F. Blake, MD, G.D. Blake, OD, James Cook University, Townsville City, Australia

Innovation concept: The slit lamp is a powerful tool for examining the eye and performing procedures. Most Emergency Physicians are daunted by all the knobs and different lenses and do not use the slit lamp to its full potential. Methods: A program was designed to teach slit lamp use focusing on corneal foreign body removal. The program was successful and now includes advanced skills such as assessment of the anterior chamber and angle measurement. Curriculum, tool, or material: The initial education program focused on using the slit lamp and corneal foreign body removal. A Power Point presentation was developed and a small accompanying booklet. A Styrofoam head with jelly eyes was used for practice. The program has evolved and now uses a Topcon slit lamp with a digital camera. This allows for ease of view- 
ing while demonstrating the use of the slit lamp and the ability to guide the learners as they perform the skills. An introductory PowerPoint presentation focuses on knob and lensology, eye vital signs, basic use of the slit lamp, corneal foreign body removal and fluorescein staining. An advanced session includes assessment of the anterior chamber, angle measurement, Seidel's sign and use of the red free filter. A MacBook was designed as a supplementary tool for easy review of the slit lamp use after the initial teaching programs. Conclusion: This "hands" on approach to teaching the slit lamp has been a success in our department with the confidence of our consultants, interns, residents and registrars substantially improving. The comment "now I get it" is common during the teaching sessions. Written descriptions and on-line videos are available but the true learning occurs only with the doing.

Keywords: innovations in EM education, slit lamp, corneal foreign body

P010

Cellulitis management within the emergency department at Regina General Hospital

K.N. Bonkowski, R. Nesbitt, MD, E. Karreman, PhD, University of Saskatchewan, Saskatoon, SK

Introduction: There is considerable variation in management of cellulitis with IV antibiotics in the Regina General Hospital (RGH) emergency department (ED). This study aims to identify when initial followup is occurring for patients placed on IV treatment for cellulitis and whether an initial follow-up request at 24 hours versus 48 hours or more results in variation of total time on IV antibiotics or in antibiotic or dosage change. Ideally, patients should not have follow-up at 24 hours, as worsening erythema may occur after antibiotics are started and may be mistaken for a poor response to antibiotics. Methods: A retrospective chart review of 57 male and 32 female patients (mean age: $48.7 \pm$ 17.8 years old) presenting to RGH ED with cellulitis and receiving IV antibiotics from June 2011 to March 2013 was performed. Exclusion criteria were pregnancy and being under the age of 16 . The main measured variables were length of time to initial follow-up request in hours, total length of time on IV antibiotics, and antibiotic or dosage change after initial follow-up. Results: Of the patients for which the necessary data could be collected from the charts, 31 had an initial follow-up request of 24 hours and 36 had an initial follow-up request of 48 or more hours. A chi-square analysis revealed that when patients had an initial follow-up request of 24 hours, they stayed on antibiotics significantly longer after initial follow-up than patients who were followed-up after 48 hours or longer ( $65 \%$ vs $36 \%, \mathrm{p}=.02)$. Of patients who had an initial follow-up at 24 hours, 8 patients $(26 \%)$ had an antibiotic or dosage change. Of those followed-up at 48 hours or more, 4 patients $(11 \%)$ had an antibiotic or dosage change $(p=.11)$. Conclusion: There is considerable inconsistency in follow-up recommendations in the ED for IV cellulitis management. The length of time to initial follow-up impacts the duration of antibiotic treatment. When initial follow-up is only 24 hours, patients are more likely to stay on IV antibiotics longer after their initial follow-up as compared to patients who are seen after 48 or more hours. Therefore, it is more efficient to delay follow-up until at least 48 hours after IV initiation because most patients will require IV antibiotics for more than 24 hours. This project has the potential to standardize treatment of cellulitis in order to decrease visits to the ED, increase patient satisfaction, and improve outcomes.

Keywords: cellulitis, IV therapy, follow-up

P011

Bag versus catheter for urine collection in infants at an academic tertiary care centre

M. Brine, MD, S.L. McLeod, MSc, M. Klingel, MSc, A. Shah, MD, G. Joubert, MD, University of Western Ontario, London, ON
Introduction: Previous pediatric literature has examined the effectiveness of bag versus catheter sample collection and has found bag urine collection to be more sensitive for detection of urinary tract infection (UTI) compared to catheter samples, however consistently less specific. The objective of this study was to determine clinical practices for urine sample acquisition in the pediatric emergency department (ED) of an academic tertiary care centre. Methods: This was a retrospective medical record review of a random sample of $121 \mathrm{ED}$ visits for patients aged $\leq 12$ months presenting to the pediatric ED of an academic tertiary care centre with a chief complaint of fever, vomiting, or unwell over a one-year period. Visits were excluded if triaged CTAS 1 or if patient had known renal disease or history of UTI. Charts were reviewed to determine if urinalysis was obtained, by what method, if catheterization was preformed after initial bag specimen and if method of collection affected ED length of stay. Results: 103 patient encounters met inclusion criteria. Urinalysis was performed in $66(64 \%)$ patients. Catheterization was the initial method of collection in 56 (84\%) patients (19 [90.5\%] ages 0-3 months, 19 [90\%] ages 3-6 months, and 19 [75\%] ages 6-12 months). Of 10 (15\%) urine samples initially collected by bag, repeat samples by catheterization were obtained in $6(60 \%)$. Of 56 samples sent for culture, $13(23 \%)$ grew organisms (3 [23\%] initially collected by bag). Median (IQR) ED length of stay was $2.7(1.9,5.1)$ hours in the catheter group and 2.2 $(1.1,2.5)$ hours in the bag group. Conclusion: Catheterization for urine sample collection was the most common method utilized for infants $\leq$ 12 months at this institution. Most samples collected by bag required a subsequent catheterization. However, sample collection by catheterization did not shorten ED length of stay.

Keywords: paediatrics, urinary tract infection

P012

An undergraduate point-of-care ultrasound interest group C. Byrne, BSc, D. Mainprize, BSc, M. Clemente, BSc, R. McLarty, BSc, N. Packer, BSc, D. Thompson, MD, R. Arntfield, MD, University of Western Ontario, London, ON

Innovation concept: Despite growing evidence and enthusiasm supporting the value of point-of-care ultrasound (PoCUS) in undergraduate medical education, training opportunities remain limited. The ultrasound interest group (USIG) has previously been shown to be a feasible model for promoting ultrasound education and leadership among medical students. This abstract describes the development of a novel PoCUS interest group within a medical student club system. Methods: In partnership with local physician expertise, a PoCUS interest group was established within the medical student club system and promoted through multiple channels. A curriculum including both didactic learning and scanning opportunities was proposed. The primary goal of the group was to introduce medical students to the fundamental principles and clinical applications of PoCUS. Curriculum, tool, or material: The interest group conducted eight sessions during the academic year, with attendance governed by availability of equipment and expert instructors. Participants were directed towards previously developed e-learning resources and session objectives in advance of relevant sessions. The sessions included six interactive ultrasound workshops 90 to 120 minutes in duration (abdominal aorta; hepatobiliary and renal; cardiac; lung and pleura; obstetrical and gynecologic; procedural guidance) and two large group discussions (introduction to PoCUS; PoCUS in medical education), one of which was conducted and archived via online broadcast. Group membership reached 106 pre-clerkship students ( $31.0 \%$ of student body) in its inaugural year. In the current year, membership has grown to 160 pre-clerkship students (46.8\% of student body). Conclusion: The large number of interest group members and sustained participation through workshops and didactic discussions supports the feasibility of student-faculty partner- 
ships in providing PoCUS training opportunities for medical students. Our one-year experience demonstrates that undergraduate medical students are engaged in extracurricular PoCUS education.

Keywords: innovations in EM education, ultrasound, medical students

\section{P013}

The utility of routinely measuring serum electrolytes as part of the investigation of patients with cardiac-type chest pain

S.G. Campbell, MB BCh, S. Field, MB BCH, K. Magee, MD, I. Cajee, MB BCH, M. Butler, BSc, C.L. Campbell, MB BS, Dalhousie University, Halifax, NS

Introduction: Many laboratory tests are ordered in ED patients as a matter of 'routine'. Many of these are of questionable value to improving patient outcome, yet add significant cost to the system. One such group of tests is of 'serum electrolytes'(SE). We examined the subset of patients with chest pain referred to cardiology to see whether the routine use of SE testing contributed to patient care. Methods: The medical records of 984 ED patients who had been referred to cardiology for the investigation of chest pain were reviewed. Clinical details and SE results were reviewed independently by four experienced ED faculty (average 25,5 yrs experience, range 18-37). Reviewers indicated if they thought the result was relevant to ED care or not. A result was considered 'relevant' if any of the reviewers considered it so. Results: 41 patients $(4.1 \%)$ had SE results considered relevant by any one of the reviewers. OR of relevant SE was 6.0 in patients with a history of previous MI. The OR of relevant SE increased lower blood pressure (OR 3.1 for SBP $<100$ vs. $>100 \mathrm{mmHg}$ ). Further logistic regression analysis will be performed in an effort to identify patient variables associated with relevant SE results. Conclusion: Routine SE testing adds little to patient care for patients with chest pain of suspected cardiac origin. Further analysis may identify patients most likely to benefit from SE. Keywords: electrolytes, chest pain

Keywords: electrolytes, routine investigation, chest pain

\section{P015}

Offload zones in the emergency department to mitigate emergency medical services (EMS) offload delay: a process map and hazard analysis

A. Carter, MD, J. Gould, BSc, P. Vanberkel, PhD PEng, J.L. Jensen, BSc, J. Cook, MD, S. Carrigan, MHA, M. Wheatley, A. Travers, MD, MSc, EHS, Halifax, NS

Introduction: Offload delay is a prolongation of the interval between ambulance arrival in emergency department (ED) and transfer of patient care. This reduces the availability of ambulances for emergency response in the community. Offload Zones $(\mathrm{OZ})$, which can receive multiple ambulance patients waiting for an ED bed and release paramedics from the ED, have been implemented as a possible mitigation. The objectives of this study were to process map the functioning of the $\mathrm{OZ}$ and conduct a hazard analysis to identify steps that could compromise patient safety or process efficiency. Methods: A Health Care Failure Mode and Effect Analysis was conducted. All major processes and steps were identified by observation and expert opinion. Hazard analysis was conducted by focus group. Failure modes (FM) were identified for each step. For each FM a probability to occur (1-4) and severity of impact on patient safety and process efficiency (1-4) was determined and a hazard score (probability X severity: $1-16$ ) was calculated. Any hazard score $\geq 8$ was considered 'high risk'. Root causes were identified for all 'high risk' FM. A hazard score was calculated for those causes. Mitigations were sought for 'high risk' causes with no control measure. Results: The OZ consists of 6 major processes: (1) Patient transported by ambulance, (2) Arrival in ED, (3) Transfer of patient Care, (4) Patient assessment in OZ, (5) Patient care in $\mathrm{OZ}$ and (6) Patient transfer out of OZ to ED. There were 110 steps within these major processes categorized as decisions $(n=13,11.8 \%)$, skills $(n=29$, $26.4 \%)$ and tasks ( $=68,61.8 \%) .78$ FM were identified, of which 28 $(35.9 \%)$ were 'high risk': patient safety $(\mathrm{n}=7 / 28,25.0 \%)$, process efficiency $(n=10 / 28,35.7 \%)$, and both $(n=11 / 28,39.3 \%)$. High scoring FM were lack of equipment (safety) and failure to move patient to ED bed (process). 57 causes for 'high risk' FM were identified of which 37 were 'high risk' with no control measure. 17 mitigations were suggested for those 'high risk' causes with no control measure. Conclusion: This process map and hazard analysis has prospectively identified a number of potential failures of the OZ. The results from this study will inform current policy and practice, and future work to understand the use of the $\mathrm{OZ}$ to reduce offload delay.

Keywords: emergency medical services, emergency overcrowding

P016

A novel approach to the assessment of alcohol withdrawal in the emergency department

S.M. Carver, BSc, N. Norouzi, BEng., S. Bromberg, BASc, S.H. Gray, MD, M. Kahan, MD, P. Aarabi, PhD, B. Borgundvaag, PhD, MD, Mount Sinai Hospital, Toronto, ON

Introduction: Alcohol withdrawal syndrome is potentially life-threatening. It is commonly encountered and poorly managed in the Emergency Department (ED). A symptom-guided approach to the treatment of alcohol withdrawal has the potential to improve care of these patients in the ED by reducing the total dose of benzodiazepine administered, reducing complication rates such as seizures while in the ED and after discharge, and potentially reducing the length of stay for these patients. Symptom-guided tools, such as the CIWA-Ar, are not routinely used because they are time-consuming, subjective, and amenable to manipulation by benzodiazepine-seeking patients. The most universally accepted clinical measure of alcohol withdrawal severity is tremor, the assessment of which is subjective and variable. We developed an iOS CIWA-Ar mobile application ('app') which standardizes tremor assessment using the phone's accelerometer. A secondary question was whether it is possible to discriminate a real alcohol withdrawal tremor from an intentional attempt to deceive the assessor. Methods: Data were collected from 10 patients in alcohol withdrawal and 12 nurses mimicking an alcohol withdrawal tremor. Assessments were videotaped and compared against "gold-standard", subjective assessments of three expert clinicians according to the CIWA-Ar scale. We used time-frequency analysis to characterize the tremors, specifically to differentiate real from factitious tremors, and to calibrate tremor intensity. Results: Real alcohol withdrawal tremors had a mean frequency of $8.1 \mathrm{~Hz}$ and a standard deviation of $1.2 \mathrm{~Hz}$. By rejecting tremors with an average frequency below $7 \mathrm{~Hz}$, real tremors could be detected with $16 \%$ false positives and $13 \%$ false negatives. Additionally, there was a general linear relationship between the CIWA-Ar tremor score and the energy of the signal such that the higher the CIWA-Ar tremor rating, the higher the energy in the $7-16 \mathrm{~Hz}$ range. Conclusion: It is possible to both quantify tremor severity and differentiate most real and fake alcohol withdrawal tremors. Ongoing work will further characterize the tremor of alcohol withdrawal and refine the calibration of the app.

Keywords: alcohol withdrawal, tremor, emergency department

P018

The McMaster Modular Assessment Program (McMAP) improves quality of in-training evaluation reports via aggregated workbased assessments and guided narrative global assessment T.M. Chan, MD, J. Sherbino, BSc, MD, MEd, McMaster University, Hamilton, ON 
Introduction: The McMAP system contains a wide variety of workplace based assessment (WBA) tools that are assembled into CanMEDS-based units, while longitudinally assessing global functioning. At the end of block, the assessments are compiled into a score report for supervising faculty members, who then complete a guided descriptive, narrative overview of the resident's performance (the McMAP intraining evaluation record, ITER). Methods: 41 McMAP ITERs written by 25 faculty members (out of a possible 64 McMAP ITERs) were available. We rated the quality of our McMAP ITERs to a historical cohort of random ITERs prior to our pilot launch. A random cohort of 25 McMAP ITERs and a matched historical cohort of 25 ITERs from PGY1 \& 2 residents from the pre-McMAP period were randomly selected. A program administrator redacted all ITERs to anonymize the documents. All 50 ITERs (both pre- and post-McMAP) were scored by two investigators (TC, JS) using the Completed Clinical Evaluation Record Rating (CCERR). Results: The level of agreement between our two raters on the CCERR scale was alpha $=0.916(\mathrm{df}=49, \mathrm{p}<0.001)$. There was a significant increase $(\mathrm{p}<0.001)$ in the median CCERR scores after the McMAP was introduced, resulting in a 15.7 point jump (out of the 45 point CCERR scale). The median CCERR score of our McMAP ITERs was 27.5 (IQR 20.5-23.5), whereas our historical cohort was 13.8 (IQR 11.3-15.8). Conclusion: Our McMAP ITER system generated significantly improved quality assessments, as judged by the CCERR scale. Summarized data and scaffolded questions can greatly improve ITER quality.

Keywords: residency education, assessment, competency-based medical education

\section{P019}

Improving emergency department care for Acute Exacerbations of COPD

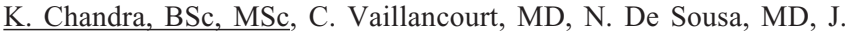
Fraser, BN, J. Scoville, P.R. Atkinson, MD, Dalhousie University, St. John, NB

Introduction: Chronic obstructive pulmonary disease (COPD) is a progressive respiratory disorder. It includes periods of acute exacerbations (AECOPD) where symptoms like breathlessness deteriorate. Current guidelines recommend treatments to relieve symptoms of AECOPD and decrease treatment failure and future exacerbations. We hypothesized that implementation of local guidelines will increase emergency department (ED) physician, nursing and respiratory therapists' (RT) awareness, knowledge and use of COPD guidelines and be related to the improved outcomes in AECOPD patients. Methods: This study was conducted at a tertiary hospital emergency department. Local COPD guidelines were developed by the quality improvement group. Copies of the guidelines were circulated and were posted in the department. A series of educational sessions were provided for physicians, nurses and RTs, and sign off was required. ED staff awareness, knowledge and use of local COPD guidelines were assessed with a survey before guideline implementation, several months after and at a tenmonth follow-up. Results: For emergency physicians, the survey response rate was 78,79 and $58 \%$ at pre-implementation, post-implementation and at 10-month follow up, respectively. Before local guideline implementation, three physicians out of 21 (14.3\%; 95\% CI 4.1$35.5 \%)$ were aware of local COPD guidelines. After guideline implementation, 20 of $22(90.9 \% ; 71.0-98.7 \%)$ were aware. At 10month follow-up, 15 of 15 physicians $(100 \%$; 76.1-100\%) were aware of local guidelines. For physician guideline use, zero physicians $(0.0 \%$; $0.00-18.2 \%$ ) used local guidelines before implementation. After guideline implementation, 18 physicians $(81.8 \%$; 60.9 to $93.3 \%)$ used guidelines. This was maintained at 10 -month follow-up with all 15 physician respondents $(100 \%$; $76.1-100 \%)$ using local COPD guidelines. Similar trends were seen among nurses and RTs though survey response rates were much lower. Conclusion: Our introduction of local COPD guidelines was successful at increasing awareness, knowledge and use of best practice guidelines among physicians, nurses and RTs in the ED. At ten-month follow-up, we maintained increased awareness, knowledge and use among ED staff. Future studies will focus on highlighting whether increased awareness and use of guidelines result in a decrease in treatment failure rates and exacerbation frequency in AECOPD patients presenting to our ED.

Keywords: guidelines, COPD, quality improvement

\section{P020}

Initial presentations and outcomes of necrotizing infections: a retrospective chart review

J. Chen, MD, M. Klingel, MSc, S.L. McLeod, MSc, V. Ng, MD, University of Western Ontario, London, ON

Introduction: Timely diagnosis, antibiotic therapy, and aggressive surgical debridement of affected tissue are crucial to the successful treatment of necrotizing fasciitis. However, necrotizing fasciitis is often initially misdiagnosed in the emergency department (ED) as a more benign skin or soft-tissue infection. The objective of this study was to determine the clinical features at presentation of patients diagnosed with necrotizing fasciitis, and the in-hospital outcomes associated with this disease. Methods: This was a retrospective medical record review of adult patients with a hospital discharge diagnosis of necrotizing fasciitis at one of two academic tertiary care EDs (annual census 125,000) over a five-year period (April 2008 to March 2013). Both ED and in-patient encounters were included. Patients transferred directly from another facility for specialty treatment were excluded. Charts were reviewed for patient demographics, co-morbidities, clinical features at presentation, microbiology, treatment and final outcome. Results: 73 patients were included. Mean (SD) age was 54.6 (17.2) years, $56.2 \%$ were male and the median (IQR) hospital length of stay was $11.2(6.0,31.2)$ days. Common co-morbidities at presentation included immunocompromise (49.3\%), diabetes mellitus (41.1\%) and obesity (31.7\%). $57.5 \%$ of patients had initial presentations of swelling and/or erythema, and $16.4 \%$ of patients had additional symptoms of bullae, petechiae, or bruising. 33 (45.2\%) patients received antibiotics. $62(84.9 \%)$ patients had wound cultures; of which $83.9 \%$ were positive. $59(80.8 \%)$ underwent surgery, with a median (IQR) time from initial presentation to surgery of 12.5 hours $(7.1,57.0)$. In-hospital mortality amongst those who had surgical intervention was $18.6 \%$, compared to in-hospital mortality of $35.7 \%$ for those who did not receive surgical intervention. Conclusion: Emergency physicians are often the first to evaluate patients with necrotizing fasciitis and as such must be aware of the presentation and management of the disease. This study suggests that diabetes mellitus, immunocompromise, and obesity are common co-morbidities of necrotizing fasciitis, and that survival may be higher amongst patients who receive surgical treatment. Patients presenting to the ED meeting this clinical picture warrant a high degree of suspicion to avert potentially disastrous consequences. Keywords: necrotizing fasciitis, infection, emergency medicine

P021

Describing current practice patterns for the management of discharged emergency department patients with hypertension: a multicentre survey

D.D. Cho, MD, P.C. Austin, PhD, C.L. Atzema, MD MSc, University of Toronto, Division of Emergency Medicine, Toronto, ON

Introduction: A large number of patients come to the emergency department (ED) for asymptomatic hypertension; these numbers will likely increase in the future. Given limited evidence to guide the management of these patients, the practice of one's peers provides an alter- 
native standard. This study sought to describe current practice patterns for the ED management of asymptomatic hypertension. Methods: In this prospective study, a standardized, piloted survey was distributed to emergency physicians during academic rounds at 3 community and 4 tertiary EDs. The primary outcome measure was the proportion of respondents who had a blood pressure (BP) threshold at which they would offer a new antihypertensive prescription or increase the dose of an existing prescription. Secondary outcomes included predictors of initiating or increasing an antihypertensive given clinical vignettes of a 65 year-old male with 2 levels of hypertension (160/100 vs. 200/110 mm $\mathrm{Hg}$ ) and increasing number of comorbidities, as well as the recommended number of days to follow-up with a primary care provider. Results: All 81 surveys were completed (response rate 100\%). Among respondents, mean number of years in practice was 11.5 (SD 10.0) and a similar proportion held certification through the College of Family Physicians vs. the Royal College. Half (51.9\%; 95\% CI 40.5-63.1) indicated that they had a systolic BP threshold for initiating an antihypertensive, and 55.6\% (95\% CI 44.1-66.6) had a diastolic threshold: mean systolic BP was $199 \mathrm{~mm} \mathrm{Hg}$ (SD 19) while mean diastolic BP was 111 mm Hg (SD 8). Similarly, half (55.6\%; 95\% CI 44.1-66.5) indicated that they had a systolic BP threshold for increasing the dose of a preexisting antihypertensive: mean systolic and diastolic BP was 193 (SD 22) and $110 \mathrm{~mm} \mathrm{Hg}$ (SD 9), respectively. A higher BP (OR 13.3; 95\% CI 7.3-24.2) and more comorbidities (OR 3.0; 95\% CI 2.1-4.4) were associated with offering an antihypertensive prescription. Respondents recommended follow-up care within a median 7.0 and 3.0 days for the patient with lower and higher BP levels, respectively. Conclusion: Half of surveyed emergency physicians reported having a BP threshold to start or increase an antihypertensive; higher BP levels and more comorbidities modified their decision. The other half of respondents reported having no threshold. In the absence of evidence, the practice of one's peers forms the next best guideline: whether the variation in practice impacts long-term morbidity or mortality is unknown.

Keywords: hypertension, emergency physicians, practice patterns

\section{P022}

Pre-hospital management of patients with history of asthma and talc lung in one Canadian urban setting

T. Chung, T. Tamura, A. Kabaroff, MD, K. Liss, BSc, C. Villa-Roel, B.H. Rowe, MD, MSc, University of Toronto, Toronto, ON

Introduction: TALC lung is a complication of chronic drug abuse that presents with respiratory distress. The early differentiation between asthma and TALC lung presentations may have an important impact on their outcomes. This study characterized patients seen at the prehospital setting with acute respiratory distress and compared initial management of patients with TALC lung. Methods: Electronic patient care records (PCRs) from the Edmonton Zone emergency medical services (EMS) in 2012 were retrospectively reviewed to identify calls made by adults ( $<55$ years) for acute respiratory distress. Data were collected by trained staff using the REDCap data platform. Descriptive (proportions and medians, interquartile ranges [IQR]) and bivariable analyses were performed. Results: From 457 PCRs, 191 (42\%) reported asthma and $79(17 \%)$ reported TALC lung history. Female sex $(58 \%$ vs. $77 \%)$ and an older population (36 vs. 46 years) were more common in the TALC lung group. Paramedics administered short acting beta-agonists (98\% vs. $96 \%)$, short acting anticholinergics $(77 \%$ vs. $78 \%$ ), oral corticosteroids ( $33 \%$ vs. $30 \%$ ) and magnesium sulphate ( $14 \%$ vs. $10 \%$ ) similarly to both groups. More patients with TALC lung required oxygen therapy ( $67 \%$ vs. $80 \%)$, cardiac monitoring $(56 \%$ vs. $73 \%$ ), and were transported to the hospital ( $84 \%$ vs $94 \%$ ) after EMS treatment. Conclusion: TALC lung exacerbations are a unique subset of patients with respiratory distress in EMS and their treatment is poorly investigated. Patients with TALC lung appear to be older and more often female than patients with asthma; however, their treatments are similar in the pre-hospital setting.

Keywords: emergency medical services, respiratory distress

P023

Pre-hospital management of acute respiratory distress in one Canadian urban setting

T. Chung, T. Tamura, A. Kabaroff, MD, K. Liss, BSc, C. Villa-Roel, B.H. Rowe, MD, MSc, University of Toronto, Toronto, ON

Introduction: Acute respiratory distress in the emergency medical services (EMS) environment is a complex presentation involving a variety of potential cardio-respiratory conditions. This study documented the diagnostic groups and interventions applied to acute respiratory distress in an urban Canadian pre-hospital setting. Methods: Electronic patient care records (PCRs) from the Edmonton Zone EMS in 2012 were retrospectively reviewed to identify calls for acute respiratory distress and record treatments. Data were collected by trained staff using the REDCap data platform. Descriptive data are reported using proportions and medians with interquartile range (IQR). Results: From 457 PCRs, the most common diagnosis was asthma (50\%), previous infections and chronic obstructive pulmonary disease (both 17\%); "other" diagnoses represented $8 \%$ of the study population. Most patients were female (61\%) and the median age was 44 years (IQR: 31 , 49). Home was the most common pick-up location and the median time from onset to EMS arrival was 9 minutes (IQR: 7, 12). Self-medication was recorded in $173(38 \%)$ events; of these $85(49 \%)$ received short acting beta agonists (SABA). Paramedics administered SABA (97\%), short acting anticholinergics $(78 \%)$, oral corticosteroids $(28 \%)$ and magnesium sulphate $(11 \%) ; 54$ patients $(11 \%)$ received other treatments (most common-epinephrine [22/54]). The median time to first respiratory treatment was 16 minutes (IQR: 12,24$)$. Most patients (91\%) were transported to the hospital with a median time of 41 minutes (IQR: 32, 53) since onset. Conclusion: Overall, paramedics in this region demonstrated high and prompt use of respiratory medications at the scene.

Keywords: emergency medical services, respiratory distress

\section{P024}

Geographic variation in sedation choice for electrical cardioversion in Canadian academic emergency departments

D. Clinkard, MSc, I.G. Stiell, MD, MSc, C.M. Clement, R.J. Brison, MD, B.H. Rowe, MD, MSc, P. Dorian, MDCM, MSc, B. Borgundvaag, $\mathrm{PhD}$ MD, D. Redfearn, MD, D. Eagles, MD, T. Langhan, MD, E. Lang, MD, K. Magee, MD, R. Stenstrom, MD, PhD, S. Rose, MD, G. Wyse, MD, PhD, J.J. Perry, MD, MSc, D. Birnie, MD, G. Wells, $\mathrm{PhD}$, A. McRae, MD, Queen's University, Kingston, ON

Introduction: Introduction: Electrical cardioversion (EC) is a short, potentially painful procedure commonly performed in the emergency department (ED). There are currently no evidence-based guidelines for medication choice for procedural sedation for EC. We sought to quantify sedation medication choices for EC at eight Canadian EDs. Methods: Methods: A secondary analysis of patients undergoing EC in two temporally separated cohorts of adults presenting with symptomatic atrial fibrillation or flutter to Canadian Academic EDs . The first cohort consisted of 562 patients undergoing cardioversion at 6 hospitals (FMC, KGH, MSH, OHCC, OHGC, UAH) from Jan 1st, 2008 to Dec 31 st, 2008. The second cohort consisted of 420 patients at 8 hospitals (FMC, KGH, MSH, OHCC, UAH, JGH, QEII and SPH) from June 28th, 2010, to April 19th, 2012. Variation in medication choices, between sites and over time were quantified using descriptive statistics 
with $95 \%$ confidence intervals. Results: Results: Of the 999 patients who underwent EC, 982 had sedation procedure documented. Mean age was 59.8 (range 18-97), 67.9\% were male, average HR 121 (50 to 237), and SBP of 130 (174 to 234). The combination of Propofol and Fentanyl was most frequently used $(57.5 \%$ (95\% C.I $54.4-60.6 \%)$ ), and has increased by $11 \%$ between the two time periods under study. Propofol alone was the next most frequently used $(33.3 \%$ (95\% CI $30.4-36.3 \%)$ ), and has decreased by $4.1 \%$ over that interval. There was substantial interhospital variability in sedation medication choices. Propofol and fentanyl use ranged from $11.1 \%$ to $63.0 \%$ in 2008 and $33.3 \%$ to $80.0 \%$ in 2010 to 2012 Conclusion: Conclusion: Sedation medication choices for EC vary substantially between EDs. Drug choice has also exhibited substantial change over the past 5 years, with practitioners coming to prefer the combination of propofol and fentanyl compared to propofol alone.

Keywords: RAFF, cardioversion, sedation

\section{P025}

Changing pain management practices in a pediatric ED: teamwork works!

E. D. Trottier, MD, N. Ferlatte, N. Gaucher, MD, MSc, M. Martineau, A. Canuel, M. Sanchez, MD, C. Hogue, MSc, M. Certain, S. LeMay, $\mathrm{PhD}$, J. Gravel, MD, MSc, Urgence Pédiatrique, CHU Ste Justine, Université de Montréal, Montréal, QC

Introduction: Pain control in pediatric emergency department (PEDs) is often suboptimal, but quality improvement (QI) initiatives to change healthcare providers' practices are often fraught with difficulties. Objective: To develop a multidisciplinary team approach to the management of pain in a PED, in order to improve procedural pain control. Methods: In spring 2013, an interdisciplinary group of ED staff (nurses, physicians, administrative) from a tertiary care pediatric university hospital was created to explore pharmacological and non-pharmacological opportunities to improve pain relief in ED patients. Frequent, brief meetings - in collaboration with hospital pharmacists, pain management researchers, and the hospital's pain team - allowed for the identification of barriers to pain management, areas needing improvement and easy, low-cost solutions that would be acceptable with minimal staff resistance. To assist these practice changes, one nursing team was identified as test group, facilitating feedback about chosen methods and enhancing both the pain team's and the staff's capacity to adapt to barriers to implementation. During the trial period, structured written feedback (quantitative and qualitative) was provided for every intervention to guide choices. Training regarding new approaches was then provided to all PED physicians and nurses. Venipuncture was chosen as the first QI initiative. Results: The list below presents achieved practice changes to reduce procedural pain during venipuncture (6 months after pain team's creation). The topical anesthetic was chosen based on literature review, but also on written comments from nursing staff regarding pain control efficacy, loss of vascular anatomy and ease of use. List of successfully implemented changes in a PED Local anesthetic for IV insertion -Parental lap/sitting position for procedure -Improved low-cost distraction methods (hide/seek games on walls and ceiling, age-appropriate distraction boxes) -Implementation of sucrose protocol for infants, using table sugar Conclusion: Using an interdisciplinary approach to pain management methods allowed for the development of creative, realistic, low-cost and achievable goals in a short time span. Also, implementing the project progressively allowed for constant, immediate feedback and adjustments. Such working models could be used to create various clinical guidelines and to facilitate actual practice changes.

Keywords: pain management, pediatric emergency, quality improvement
P026

Handover in the emergency department: qualitative analysis of staff emergency physician perceptions of handover

C. Dakin, MD, E. Bidlake, PhD, A. Haligua, MD, J. Frank, MD, MA(Ed), L.A. Calder, MD, MSc, University of Ottawa, Department of Emergency Medicine, Ottawa, ON

Introduction: There have been many attempts to create Emergency Department shift change handover tools, but there has been poor uptake of these tools. This study was designed to explore Emergency Physician (EP) perceptions of handover, as well as their desire for and vision of a written handover tool. Methods: Single centre qualitative study in 2 parts. Subjects were purposely selected from a volunteer pool of EPs affiliated with the Department of Emergency Medicine at The Ottawa Hospital. Part 1: individual semi-structured interviews exploring the study objectives with 5 EPs. Part 2: 8 participant focus group with 2 goals - to triangulate the interview results, and to obtain feedback on a written handover tool draft based on observational ED data from a previous study and refined by the interview data. Transcripts were analyzed for thematic content by 2 independent researchers. Results: EPs were generally interested in trialing a tool. Themes clustered around effective elements of handover, effective elements of a tool, barriers to handover, and barriers to a tool. The tool needed to be concise, but still include pertinent information by headings - most importantly the action plan. It also needed to include all active patients in the department. Handover was most effective when it included collaboration on diagnostic or management uncertainties. Major barriers to the tool were the time pressures, the patient variability in requirements, and legibility. Major barriers to handover also included 'pushback' from on-coming EPs, poor communication especially regarding non-medical aspects or diagnostic/management uncertainty, and time pressures. When shown a draft, most focus group participants desired a tool with more open space and room for customization. Conclusion: There are consistent themes relating to desirable elements and barriers to both handover and a tool during discussion with EPs. Most EPs were interested in trialling a written tool. Keywords: handover, patient safety

P027

Corticosteroids and antihistamines in the treatment of anaphylaxis: a systematic review

G. Dolansky, MD, S. Calder-Sprackman, MD, A. Plint, MD, R. Zemek, MD, Children's Hospital of Eastern Ontario, Ottawa, ON

Introduction: The wide variability in prescribing patterns and adherence to guidelines for the acute management of anaphylaxis may be partially justified by the paucity of randomized controlled trials examining the efficacy of pharmacological treatment. The objective of this study was determine if the use of epinephrine, corticosteroids or antihistamines affect rates of biphasic reactions in patients presenting to the emergency department (ED) with anaphylaxis. Methods: We searched Medline (1946-September 2013) and EMBASE (1974-September 2013) using a peer-reviewed search string to identify original, English-language studies describing pharmacological intervention for patients of all ages presenting to the ED with anaphylaxis. Exclusion criteria included single case reports, review articles, or treatment not reported. The primary outcome was the association of pharmacological treatment with biphasic reaction. Secondary outcome was the adherence to recommended guidelines for the use of pharmacotherapy in anaphylaxis. Results: Of 1424 citations, 1153 remained after removal of duplicates. After dual review, 35 observational studies remained. Twenty-seven studies were included in qualitative analysis, and seven studies were included in meta-analysis (pooled $n=1471$ ). Qualitative 
analysis revealed that current anaphylaxis guidelines are not being followed, regardless of population age or location. Corticosteroids and antihistamines are consistently prescribed more often than epinephrine. Meta-analysis revealed no significant association between of any of the medications studied and biphasic reactions. Conclusion: Minimal evidence supports the use of adjunctive therapy to prevent biphasic reaction for anaphylaxis. There is poor application of existing guidelines for the use of epinephrine in anaphylaxis, highlighting a role for further knowledge translation. Prospective, controlled clinical trials are needed to determine the role for adjunctive medications (corticosteroids, antihistamines) in anaphylaxis.

Keywords: anaphylaxis, corticosteroids, antihistamines

P028

Complications increase with greater than one endotracheal intubation attempt: experience in a Canadian adult tertiary-care teaching centre

L.V. Duggan, MD, K.S. Minhas, BSc, D.E. Griesdale, MD, T.L. Miller, BSc, J. Zurba, BSc, B. Deady, MD, R. Noseworthy, MD, L.V. Carter, BA, S.C. Reynolds, MD, Departments of Anesthesiology, Emergency Medicine, and Intensive Care Medicine, Royal Columbian Hospital, New Westminster, BC

Introduction: A prospective review was undertaken to better understand out-of-operating room endotracheal intubation (ETI) procedures performed throughout a 402-bed Canadian adult tertiary-care teaching center. Methods: The circumstances, methods and complications of ETI were collected over an 11-month period (July 2012-May 2013). Respiratory therapists documented information throughout ETI using data-recording cards. To estimate data-capture rate, data cards were compared to hospital records of all admitted, ventilated patients during this time. Results: Data regarding 271 patients undergoing ETI was obtained, accounting for $70 \%$ of patients admitted to hospital who required mechanical ventilation. However, this may not accurately reflect our data-capture rate; not all admitted and ventilated patients underwent ETI at our center, and some patients captured on our datacards either died or were extubated in the emergency department prior to admission. 182 patients required one attempt, 89 required more than one attempt. 122 (45\%) were performed by residents: staff physicians directly supervised all but four. Locations of ETI were intensive care units $(44 \%)$, emergency department (40\%) and other locations (16\%). Urgency was equally divided amongst emergent, urgent and semi-elective situations. Conclusion: Greater than one attempt at ETI was associated with a 4-fold increase in severe, and a 5-fold increase in total complications. Although previous publications found that greater than two attempts were associated with increased complications, recent papers found a similar association with greater than one attempt, consistent with our findings. This new information has implications for both teaching and decision-making of ETI.

Keywords: endotracheal intubation, complications, implications for teaching

\section{P029}

\section{Emergency department induced delirium in seniors}

M. Emond, MD, MSc, D. Grenier, MD, J.S. Lee, MD, N. Le Sage, MD, MSc, Université Laval, Québec, QC

Introduction: In 2011, the first members of the "baby-boomer" generation turned 65 y.o. By 2031, the proportion of the population greater than 65 will nearly double, with the largest increases occurring among those 85 years of age and older.[1] Care of older patients in the Emergency Department (ED) is particularly challenging. In 1999, Inouye et al. demonstrated that an ED stay of 12 hours or more was one of the strongest independent predictor of subsequent delirium in older patients. This is of increasing concern, as ED wait times is quite significant. To date no data exist on the incidence of ED-induced delirium following the recent « senior-friendly approach era ». Objectives : The primary objective of this study was to measure the Incidence and impacts of ED-induced delirium among older ED patients who have prolonged ED stays (> 12 hours). Methods: An historical cohort study was realized at a Canadian university-affiliated in Emergency Department. Eligible patients were identified using the Hospital or Emergency Department Information System patient tracking software. Inclusion Criteria were patient aged $\geq 65$ years, admitted to any hospital ward and non-delirious at ED admission. All patient were exposed to a minimal 12 hours of ED stay. Patient with delirium before arrival to ED were excluded. Primary Outcome: Incidence of ED-induced delirium was assessed with the modified Confusion Assessment Method (CAM) Secondary outcomes: the impact of the delirium incidence was measured using total length of stay (LOS) from ED admission to hospital discharge. Research assistants assessed patient records with a standardized data collection tools . Data analyses : Interobserver agreement were realized. Simple proportions were tested using the Chi-square statistic. LOS will be log-transform and linear regression assessed differences between the delirium positive and delirium negative. Adjustments were made for age and co-morbidity. Results: Two hundred patients records from 2009-2011 were randomly assessed and reviewed, 109 (55.5\%) were female, median age was 78.9 y.o. (SD : 7.3). Thirty-six (18\%) patients experienced an EDinduced delirium episode. Half of the episodes started while in ED and within $24 \mathrm{~h}$ after the exposure. Comorbidities profile was similar between delirium positive and delirium negative patient. Only 12 (33\%) patients with ED-induced delirium episode had a fracture or trauma-related diagnosis in ED. Mean LOS after adjustments was 20.5 days in the delirium positive group vs 11.9 days in the negative delirium patient $(\mathrm{p}<0.03)$. Conclusion: Our historical study assessing EDinduced delirium shows that 1 senior out of 5 became delirious after a $12 \mathrm{~h}$ stay in the ED. A delirious episode increase LOS by more than a week. Intervention for screening and preventing delirium could reduce LOS and overcrowding in the ED.

Keywords: delirium, seniors

\section{P030}

Emergency department admission rates of community and long term residential care elderly patients: a comparative study J. Eppler, MD, E. Ryan, MD, R.B. Abu-Laban, MD, MHSc, Department of Emergency Medicine, University of British Columbia, Vancouver, BC

Introduction: Frail elderly patients frequently present to the emergency department (ED) and require hospital admission. The belief that patients from long-term residential care (LTRC) facilities have higher admission rates than community dwelling elders has led to proposals to divert such patients from the ED to reduce access block. However the potential impact of proposals of this nature are poorly understood. Our objective was to comparatively evaluate the admission rates of two groups of elderly patients: those living in the community and those in LTRC. Because LTRC patients have nursing support, we hypothesized that the admission rate for this group would be lower than community dwelling elders. Methods: A medical record review of patients age 65 or over presenting to the ED of Kelowna General Hospital (KGH) in October 2012 was carried out using explicit criteria. Information captured included patient demographics, LTRC status, time to physician assessment, length of stay (LOS), disposition and mortality. Descriptive statistics were generated and a logistic regression model was fit to assess the association of the following a priori defined covariates on hospital admission: LTRC status, time to physician, gender, age, time of day, and weekday/weekend status. Research ethics board approval 
was obtained. Results: 5056 patients were seen in the KGH ED during the study period, of whom $1510(29.9 \%)$ met our inclusion criteria. The mean age of the study population was 78.6 years, $55.1 \%$ were women, and 147 (9.7\%) were from LTRC. The mean ED LOS was 6.1 hours for LTRC patients and 5.1 hours for non-LTRC patients $(\mathrm{p}=0.009)$. The 30 -day mortality was $12.2 \%$ and $4.9 \%$ for LTRC and non-LTRC patients respectively $(\mathrm{p}=0.001)$. The admission rates for LTRC and non-LTRC patients were significantly different $(44.2 \%$ vs. $32.7 \%$ respectively, difference $11.5 \%, 95 \%$ CI $3.1 \%-19.9 \%, \mathrm{p}=0.005$ ). However the multivariate model indicated only older age, shorter time to physician assessment and male gender were independently associated with hospital admission. Conclusion: ED patients from LTRC have a higher hospital admission rate than community dwelling elders. However this univariate finding does not persist on multivariable modeling. Further research is required to determine the most important factors and confounders associated with the hospital admission of frail elderly patients from the ED.

Keywords: elderly, hospital admission, residence type

\section{P031}

Smart card enabled thin clients in the emergency department environment: satisfied physicians and improved information system access

M. Erskine, MSc, MDCM, A. Roudsari, PhD, O. Shabestari, PhD, University of Calgary, Calgary, AB

Introduction: Calgary has Canada's largest integrated Department of Emergency Medicine, seeing over 250,000 patients annually at four sites. In an effort to enhance physician productivity, thin client terminals equipped with smart card readers were implemented in the spring of 2012. This project was designed to measure physician acceptance of the technology and assess its usability. Methods: An online survey employing standardized instruments was sent to 148 physicians in the Calgary zone. Results: The survey response rate was $70 \%$. Emergency physicians believed that implementation of the smart card enabled thin client system made accessing the emergency department information system (EDIS) easier. While only seven respondents (8\%) found the EDIS "easy" or "very easy" to access prior to use of the thin clients, this number improved to fifty-five people $(61 \%)$ after implementation. Users found the system to be both useful and easy to use. While emergency physicians were satisfied with the overall usability of the thin client terminals, qualitative data revealed some challenges related to the smart card interface that can be minimized with appropriate planning. Conclusion: By eliminating the need for multiple logins at multiple locations, the implementation of smart card equipped thin clients renders users' virtual desktops truly portable and persistent - important characteristics in the fast-paced, mobile environment of a hospital emergency department. This change makes accessing the existing EDIS easier, thereby improving emergency physician's perceived efficiency and productivity without the need for costly upgrades to the entire clinical information system.

Keywords: Medical Informatics, Emergency Department

\section{$\mathrm{P} 032$}

A retrospective cohort study examining treatments and outcomes for frostbite in two tertiary-care emergency departments

J.C. Fabian, MD, J.J. Perry, MD, MSc, M. Taljaard, PhD, M. Tran, S. Agaybi, BSc, University of Ottawa, Ottawa, ON

Introduction: Frostbite treatment guidelines are based on observational data from the 1960s and rational approaches based on physiology. Their effectiveness in preventing surgical intervention has yet to be examined. Our objectives were to characterized frostbite injuries in
Eastern Ontario, their associated risk factors, and assess the efficacy of current treatment guidelines in preventing operative outcomes. Methods: A historical cohort study examined patients treated for frostbite over 10 years at the two emergency departments of The Ottawa Hospital. Patient demographics and risk factors were recorded on standardized data extraction forms. Frostbite severity was categorized into superficial (1st and 2nd degree) or deep (3rd and 4th degree). Treatments provided were recorded, including adherence to guidelines (twelve), and surgical interventions. Results: Of the 265 frostbite patients identified, mean age was 37.5 years old, $74.0 \%$ were male, $93.7 \%$ of injuries occurred between November to March and $21.9 \%$ were admitted to hospital. Deep frostbite accounted for $17.7 \%$ of patients, of which $14.9 \%$ had an amputation, and $10.6 \%$ had debridement. Superficial frostbite accounted for $82.3 \%$ of patients, of which $4.1 \%$ had an amputation, and $2.3 \%$ had debridement. Risk factors included alcohol abuse (14.7\%), smoking (10.6\%), illicit drug use $(6.4 \%)$, psychiatric illness of any kind $(20.8 \%)$, schizophrenia $(9.1 \%)$, mood disorders $(6.0 \%)$, drug induced psychosis $(1.9 \%)$, suicide attempts $(1.1 \%)$, motor vehicle collisions $(1.5 \%)$, skidoo use $(0.8 \%)$, winter sports $(8.3 \%)$ and inadequate footwear $(32.1 \%)$. Of the twelve treatment guidelines, adherence of each ranged from $0.8 \%$ to $32.8 \%$ per guideline. Prevalence of amputation or debridement was $7.7 \%$ among patients not adherent to guidelines and $42.9 \%$ in patients adherent to guidelines. Conclusion: This study found that alcohol abuse, smoking, psychiatric illnesses, winter sports and poor footwear were prevalent frostbite risk factors. Frostbite treatment guidelines were not regularly followed. Amputation and debridement rates did not appear to improve when patients followed treatment guidelines.

Keywords: frostbite, treatment

P033

Development of evidence-based tiered response criteria for first responders in Ontario

M. Feldman, MD PhD, L. Turner, PhD, R. Burgess, BSc, Sunnybrook Centre for Prehospital Medicine, Toronto, ON

Introduction: Tiered response is the notification of allied emergency services (police or fire services) to provide basic life support at emergency medical calls. The clinical information obtained from the 9-1-1 lay caller is limited, but still must be used to make decisions about tiered response. The risks and costs to the community must be balanced with the potential benefits to the patient. Potential survival benefits have been clearly demonstrated in cardiac arrest, but are plausible in other emergent clinical situations. Allied emergency services are also required for a variety of non-medical reasons, such as scene safety, investigation, rescue, entrapments, fires, and hazardous materials incidents. Risks include resource limitations and risk of emergency vehicle collisions or bystander collisions (the so-called wake effect). Current tiered response criteria in Ontario are based on expert opinion from emergency medical services (EMS) physicians and dispatch experts. The objective of this study is to identify specific dispatch categories (i.e: call type and priority) using the Ontario Dispatch Priority Card Index version II likely to be associated with the need for a potentially life-saving intervention by first responders. Methods: We used a previously-published Delphi process to select 21 potentially lifesaving paramedic procedures which are indicative of the need for intervention by first responders. For example, if an emergency cricothyrotomy was performed, a first responder could have or ought to have performed a noninvasive maneuver to attempt to maintain an airway and/or ventilate the patient. We also included cases with paramedic Canadian Triage Acuity and Scale (CTAS) ratings of 1 (highest acuity) as indicative of the potential need for the assistance of first responders. We obtained a large administrative data set of ambulance dispatch records for calls dis- 
patched in the Region of Peel and the County of Simcoe between July 1, 2009, and December 31, 2009, which were linked by call number to the corresponding ambulance call reports. Records missing call type, dispatch priority, or CTAS were excluded. We also excluded call categories with less than ten calls in the data set. We searched the records for the 21 paramedic procedures which were indicative of the need for first responder intervention and produced a rank order list of dispatch categories which were associated with these procedures. Results: Our search yielded a data set of 38985 dispatch records linked to ambulance call reports. The dispatch categories most likely to be associated with the need for first responder intervention were, in descending order of frequency of need for intervention, cardiac arrest (with $43 \%$ requiring first responder intervention), allergic reaction, decreased level of consciousness/unconscious, seizure, breathing problem, primary assessment problem, and overdose/poisoning (with $2.7 \%$ needing first responder intervention). Conclusion: Using clinical findings of need for intervention by paramedics represents a novel method to determine need for tiered response. Use of evidence-based clinical criteria for recommending the need for tiered response allows the rational deployment of first responders to calls in which they are most likely to be needed for purely medical intervention, in addition to those calls in which they are needed for scene safety or rescue.

Keywords: emergency medical services, dispatch, tiered response

\section{$\mathrm{P} 034$}

Choice of smoking cessation counselling via phone, text, or email in emergency department patients

W. Fingrut, L. Stewart, MPH, K. Cheung, MD, MPH, Vancouver General Hospital, Vancouver, BC

Introduction: Tobacco smoke is the leading cause of preventable deaths in Canada. In British Columbia, QuitNow Services is a government-funded smoking cessation service that offers smoking cessation counselling via phone, text, or email. At our tertiary care academic Emergency Department (ED), approximately $60 \%$ of smokers accept referral to our provincial smoking cessation service. However, it is unclear whether certain patient factors affect patient choice of phone, text, or email. In this study, we seek to determine whether age, gender, or motivation to quit affect a patient's choice of service modality. These results will help refine our smoking cessation counselling services. Methods: All adults $\geq 18$ years of age who had used tobacco within the last 30 days prior to their ED visit and who accepted referral to QuitNow Services from November 2011 to February 2013 as part of a randomized controlled trial (ClinicalTrials.gov, NCT01454375) indicated their preference of receiving smoking cessation counselling via phone, text, or email. We performed chi-squared tests of independence to determine if patient gender or motivation to quit were associated with service modality selection. A one-way ANOVA was used to compare the mean age of patients in each group. Results: During the study period, 368 patients accepted a referral to QuitNow Services and selected one service modality. The average age of these patients was 41.7 years and $67 \%$ were male. According to the Prochaska stages of change, $78 \%$ of all patients were in the contemplation or preparation stages. In our sample, $44 \%$ chose phone, $17 \%$ chose text, and $40 \%$ chose email services. There was a significant association between age and choice of service modality. The average age for patients preferring text services (mean $=33.6$ years) was significantly lower than both the email (mean $=41.3$ years) and phone (mean $=45.1$ years) groups at the $\mathrm{p}<0.001$ level. Gender and stage of change were not associated with choice of service modality. Conclusion: Over $80 \%$ of ED smokers who accepted a referral to QuitNow Services chose the phone or email modality. The lesser chosen text referral modality was more popular with younger patients. Gender and stage of change did not affect patient choice of service modality in this study. Further research is needed to refine smoking cessation strategies; specifically, counselling services that rely on texting should be aware that they may attract a younger population.

Keywords: smoking cessation, health promotion, primary prevention

\section{P035}

Existing models of prehospital care in low and middle income countries: a descriptive systematic review of current literature L. Francis, MD, C. Vaillancourt, MD, MSc, A. Pozgay, MD, Department of Emergency Medicine, University of Ottawa, Ottawa, ON

Introduction: Organized prehospital care and safe transport can mitigate the morbidity and mortality associated with life and limb threatening conditions. Despite the rising prevalence of such conditions in low and middle income countries (LMICs), very little is known about their prehospital organization and practices. We sought to describe the current organizational status of prehospital care in LMICs to aid in the planning and development of future international prehospital care initiatives. Methods: We systematic searched MEDLINE, EMBASE, PubMed, CINAHI, and reviewed all referenced articles and reviews published from 2003-2013. Two trained investigators independently assessed papers for inclusion, quality, and data extraction using a piloted form and kappa values were calculated. We included papers describing prehospital care practices in LMICs with no restriction by language or method. Prehospital care systems were classified as: 1) transport only (TO); 2) basic first responder (FR); 3) Anglo-American (AA); or 4) Franco-German (FG) for the basis of comparison. We defined LMICs according to the classification provided by the World Bank organization in 2013. Results: Our search strategy identified 3,477 papers and 213 were selected for full review. All articles selected by at least one reviewer were reviewed in full. 161 papers (105 original research, 47 descriptive, and 9 editorials) met inclusion criteria. They described 184 prehospital care systems among 48 various LMICs. 115 papers $(62.5 \%)$ referenced upper-middle income countries (U-MIC), 48 (26.1\%) lower-middle income countries (LMIC), and 21 (11.4\%) low income countries (LIC). They had the following prehospital care organization: for U-MIC 7 (6.1\%) TO, 30 (26.1\%) FR, 36 (31.3\%) AA, 29 (25.2\%) mixed AA/FG, 22 (19.1\%) FG; for L-MIC 14 (29.2\%) TO, 15 (31.3\%) FR, 19 (39.6\%) AA, 4 (8.3\%) mixed AA/FG, 5 (10.4\%) FG; and for LIC 6 (28.6\%) TO, 13 (61.9\%) FR, 7 (33.3\%) AA, 2 (9.5\%) mixed AA/FG, and 0 FG. Conclusion: The Anglo-American system has been adopted by more than $30 \%$ of all LMICs. It is the preferred system among U-MICs and LMICs, with the exception of LICs where a basic first responder system is most common. The Franco-German system has been adopted by some LMICs, mostly among UMICs where a transport only system is also the least common. Understanding the organizational level of prehospital care in LMICs could facilitate the planning of future international collaborative efforts.

Keywords: pre-hospital care, emergency medical services, international

P036

The resident duty hours debate: do Canadian EM trainees have different work patterns and satisfaction?

J.R. Frank, MD, MA(Ed), K. Imrie, MD, L.J. Gorman, MA, A. Ronson, MSc, S. Taber, MHA, Royal College of Physicians and Surgeons

Introduction: Resident duty hours $(\mathrm{RDH})$ are being debated worldwide with respect to issues such as patient safety, resident wellness, and educational effectiveness. In 2013, the Canadian national task force on $\mathrm{RDH}$ conducted a survey of residents and others and reported a snap- 
shot of trainees' work patterns and satisfaction with training. In this subgroup analysis, we describe the patterns reported by Emergency Medicine (EM) residents versus the general population of resident respondents nationally. Methods: We created an RDH questionnaire with the input of the major medical education organizations on the task force. Residents were asked to describe their work patterns including hours worked, weekly activities, moonlighting, procedural training and satisfaction with overall training. The survey was distributed online to all active trainees in Canada $(\mathrm{n}=12672)$ via provincial housestaff organizations. Results: 3610 residents completed the entire survey $(28.5 \%)$, with 114 identifying themselves as EM trainees (3.2\%). More EM trainees, when compared to the national average, reported working less than 40 clinical hours per week $(12.3 \%$ vs $6.3 \%, p=0.035)$, and less than 15 hours consecutively $(76.1 \%$ vs $66.9 \%, \mathrm{p}=0.013)$. A greater percentage of EM residents reported moonlighting 1-20 hours per week, and fewer in EM reported moonlighting 0 or $>20$ hours $(\mathrm{p}=0.006$ ). No difference was observed in overnight call, teaching others, or supervising others. The majority of EM trainees (85.1\%) endorsed 40-69 hours per week as reasonable $(p=0.43)$. A greater proportion of EM trainees were dissatisfied with work schedules $(\mathrm{p}=0.035)$. Satisfaction with compensation, adequacy of clinical hours and procedural training, and overall career choice were similar to peers in other disciplines. Conclusion: While EM trainees appear similar to their peers in many respects, they reported working few hours, moonlight more, and had greater dissatisfaction with work schedules. Leaders in EM education should consider $\mathrm{RDH}$ policies in light of these findings.

Keywords: resident duty hours, EM education, moonlighting

P037

The resident duty hours debate: do Canadian EM trainees prefer different policy options?

J.R. Frank, MD, MA(Ed), K. Imrie, MD, L.J. Gorman, MA, A. Ronson, MSc, S. Taber, MHA, Royal College of Physicians and Surgeons

Introduction: Resident duty hours (RDH) are being debated worldwide with respect to issues such as patient safety, resident wellness, and educational effectiveness. In 2013, the Canadian national task force on RDH consulted residents and other stakeholders with respect RDH policy directions and desired reforms. However, it is not known if Emergency Medicine (EM) trainees differ in their perspectives versus their peers in other disciplines. In this subgroup analysis, we describe the options endorsed by EM residents versus the general population of resident respondents nationally. Methods: We created an $\mathrm{RDH}$ questionnaire with the input of the major medical education organizations on the task force. Residents were asked to provide their perspective on RDH with respect to national standards, fixed duty hour limits, tailoring by discipline, and needed reforms. The survey was distributed online to all active trainees in Canada $(n=12672)$ via provincial housestaff organizations. Results: 3610 residents completed the entire survey $(28.5 \%)$, with 114 identifying themselves as EM trainees (3.2\%). Both EM and other residents supported a national standard for $\mathrm{RDH}(86.5 \%$ vs $81.6 \%, \mathrm{p}=0.19)$ and duty hours that are tailored to each discipline $(68.8 \%$ vs $72.4 \%, \mathrm{p}=0.44)$. However, a greater proportion of EM trainees preferred fixed maximum duty hours over fatiguebased limits $(84.1 \%$ vs $72.3 \%, p=0.015)$. Proportionally fewer EM trainees endorsed reforming RDH via competency based education, decreasing administrative tasks, new models of care provision, or utilization of other healthcare professionals. Conclusion: While EM trainees endorsed similar RDH policy perspectives to their peers in other disciplines, they preferred fixed maximum duty hour limits and fewer new RDH strategies. Leaders in EM education should consider EM RDH policies in light of these findings.

Keywords: resident duty hours, EM education, policy
P038

Investigating the necessity of CT scans in children with headaches: a retrospective review

R. Gandhi, HBA, E.C. Lewis, MD, J. Evans, BSc, E. Sell, MD

The University of Ottawa Faculty of Medicine, Ottawa, ON

Introduction: Headaches are a common problem in the pediatric population. In 2004, The American Academy of Neurology (AAN) developed guidelines on neuroimaging for patients presenting with headache. We set out to determine the frequency of computed tomography (CT) scans ordered by Emergency Physicians, Community Physicians, and Neurologists, for pediatric patients presenting with primary headache. Methods: A retrospective chart review was conducted at the Children's Hospital of Eastern Ontario (CHEO), a tertiary care center in Ontario, Canada. One hundred fifty-one records were randomly selected from 2004-2009 for patients referred to the outpatient neurology clinic at CHEO with 'headache' or 'migraine' as the primary complaint. Ninety-nine patients were ultimately included. Results: Thirty-four percent (34/99) of patients presenting with normal neurologic exams had undergone a CT scan. None of the CT scans $(0 / 34)$ showed significant findings and none changed the headache diagnosis or management. Twenty-four percent (8/34) of CT scans were ordered by CHEO Emergency Physicians, forty-four percent (13/34) by Community Physicians, and thirty-two percent (11/34) by CHEO Neurologists. Conclusion: Physicians may be performing a high frequency of CT scans on children presenting with primary headaches and normal neurological exams, despite well established AAN guidelines regarding neuroimaging. These $\mathrm{CT}$ scans may not be impacting diagnosis and management. The medical-legal climate in Canada may be compelling physicians to over-investigate this population, increasing the risk of adverse events due to radiation exposure. Further studies are needed to examine the risk-benefit implications for performing CT scans on pediatric patients with recurrent headaches and normal neurologic examinations.

Keywords: CT scan, pediatric, headache

\section{P039}

Adenosine utilization and effect on supraventricular tachycardia in a large, urban EMS system

J.M. Goodloe, MD, A.O. Arthur, PharmD, C. Letson, J.W. Witmer, MPH MS, S.H. Thomas, MD, MPH, The University of Oklahoma School of Community Medicine, Tulsa, OK

Introduction: Emergency Medical Services (EMS) protocols commonly enable Paramedics to administer adenosine for supraventricular tachycardia (SVT). There is a paucity of literature on adenosine utilization and effect on SVT in EMS patients. This study's purpose was to focus upon how Paramedics utilize adenosine and the changes that occurred in presumed SVT treated with adenosine. The study EMS system is located in two large, urban municipalities in the southwestern United States. The study EMS system utilizes standing order protocols specifying adenosine 12 milligrams $(\mathrm{mg})$ rapid intravenous push for adult stable SVT, defined by regularly occurring, narrow QRS complexes indicating ventricular response rates exceeding 150 beats per minute (bpm), with systolic blood pressure at least $100 \mathrm{mmHg}$. Repeating adenosine $12 \mathrm{mg}$ is allowed if initial dosing ineffective in converting presumed SVT. Methods: Retrospective chart review of consecutive patient encounters involving administration of adenosine in adults (defined as 18 years of age or greater) with presumed SVT occurring January 1, 2012 to January 1, 2013 in the study EMS system. Results: In the 12 month study period, 252 adult patient encounters involved adenosine administration for presumed SVT. 153/252 (60.7\%) patients were female. Mean patient age was 54.8 years. Mean pre-adenosine 
heart rate was 187 bpm, with ECG review revealing 221/252 (87.7\%) patients to be experiencing SVT. The next most common pre-adenosine ECG rhythm was atrial fibrillation with rapid ventricular response (RVR) in 17/252 (6.8\%) patients. Protocol compliance with adenosine dosing was particularly high, 247/252 (98.0\%) patients receiving initial adenosine dosing of $12 \mathrm{mg}$. A second adenosine dose was administered in 77/252 (30.6\%) patients. Mean post-adenosine heart rate was 138 bpm, with ECG review revealing the following common post-adenosine rhythms: 100/252 (39.7\%) sinus tachycardia; 58/252 (23.0\%) sustained SVT; 49/252 (19.4\%) sinus rhythm; 28/252 (11.1\%) atrial fibrillation with RVR. 241/252 (95.6\%) of study patients were transported by EMS to an emergency department. Conclusion: In a sizeable adult EMS patient cohort receiving adenosine for presumed SVT, nearly $60 \%$ of patients were converted to a sinus rhythm with heart rates less than $150 \mathrm{bpm}$. Paramedic protocol compliance for stable presumed SVT was very high.

Keywords: emergency medical services, suprventricular tachycardia, dysrhythmia management

\section{P040}

Airway management practices in adult non-traumatic cardiac arrest in a large, urban EMS system

J.M. Goodloe, MD, A.O. Arthur, PharmD, P. Holder, MD, W. Murry, BS, J.W. Witmer, MPH MS, L. Higgins, S.H. Thomas, MD, MPH, The University of Oklahoma School of Community Medicine, Tulsa, OK

Introduction: Emergency Medical Services (EMS) care confers distinct impact upon survivability from sudden cardiac arrest. This study's purpose was to focus upon airway management practices by EMTs and Paramedics in a sizeable cohort of adults suffering nontraumatic cardiac arrest, treated in a large, urban EMS system in the southwestern United States. The study EMS system utilizes standing order protocols for airway management that enable EMTs to insert a supraglottic airway if bag valve mask (BVM) ventilation proves procedurally ineffective and that enable Advanced EMTs and Paramedics to either insert a supraglottic airway or endotracheally intubate. There is a limit of three intubation attempts per patient specified by protocol. Methods: Retrospective chart review of consecutive patient encounters involving initiation of cardiopulmonary resuscitation for adults (defined as 18 years of age or greater) with non-traumatic cardiac arrest occurring January 1, 2012 to January 1, 2013 in the study EMS system. Results: In the 12 month study period, 1,042 adult patient encounters involved cardiopulmonary resuscitation initiated by EMS professionals for non-traumatic cardiac arrest. $602 / 1,042(57.8 \%)$ patients were male. Mean patient age was 63.2 years, $79.6 \%$ patients being 50 years of age or older. BVM device utilization was documented in 976/1,042 (93.7\%) resuscitations. The supraglottic airway utilized in the study EMS system was the King LT-D, placed in 227/1,042 (21.8\%) resuscitations, successfully placed on first attempt in 219/227 (96.5\%) uses. Only 7 attempts at supraglottic airway placement proved unsuccessful within 3 attempts. Endotracheal intubation was achieved in 785/1,042 (75.3\%) resuscitations. There were 53/785 (6.8\%) instances of extubation, 37/53 $(69.8 \%)$ due to loss of continuous waveform capnography post-intubation. All patients in the cohort had documented achievement of oxygenation and ventilation. Conclusion: In a sizeable adult cohort with EMS resuscitation for non-traumatic cardiac arrest, airway management is primarily by BVM device preceding endotracheal intubation performed by Advanced EMTs and Paramedics. Nearly one quarter of studied patients had airway management involving supraglottic airway placement.

Keywords: emergency medical services, airway management, cardiac arrest
P041

Endotracheal intubation in adult, non-traumatic cardiopulmonary arrest in a large, urban EMS system

J.M. Goodloe, MD, A.O. Arthur, PharmD, P. Holder, MD, W. Murry, BS, J.W. Witmer, MPH MS, L. Higgins, BS, S.H. Thomas, MD, MPH, The University of Oklahoma School of Community Medicine, Tulsa, OK

Introduction: Controversy exists as to the benefits and risks of endotracheal intubation in cardiopulmonary arrest (CA) patients treated by Emergency Medical Services (EMS) systems. This study's purpose was to analyze the endotracheal intubation practices in a sizeable cohort of patients with non-traumatic CA encountered in a large, urban EMS system in the United States. Methods: Retrospective chart review of consecutive patient encounters involving initiation of cardiopulmonary resuscitation for adults (defined as 18 years of age or greater) with non-traumatic CA with documented attempts of endotracheal intubation from January 1, 2012 - January 1, 2013 in the study EMS system. Results: Endotracheal intubation was achieved in $785 / 1,042$ (75.3\%) non-traumatic CA patient encounters in the study period. Of the 934 patients in which multiple intubation attempts were documented, 168/934 (18.0\%) had two attempts documented, 37/934 $(4.0 \%)$ had three attempts documented, and only 7/934 (0.95) had more than three attempts documented. The maximum number of attempts documented was six. There were 53/785 (6.8\%) endotracheal tube placements that resulted in subsequent extubation, most commonly $37 / 53$ (69.8\%) due to loss of continuous waveform capnography. All cohort patients had documented achievement of oxygenation and ventilation. Conclusion: In a sizeable cohort of adults with nontraumatic CA treated by a large, urban EMS system in the United States, endotracheal intubation attempts were primarily limited to one or two, until either intubation was successful or alternative airway management strategies were pursued.

Keywords: Emergency Medical Services, Airway Management, Cardiac Arrest

\section{P042}

Presentation of acute allergic reactions in a large, urban EMS system

J.M. Goodloe, MD, A.O. Arthur, PharmD, B. Cory, MD, W. McNitt, BS, S.H. Thomas, MD, MPH, The University of Oklahoma School of Community Medicine, Tulsa, OK

Introduction: Emergency Medical Services (EMS) systems are wellpositioned to deliver effective, timely interventions for the spectrum of allergic reaction acuities. Due to the variety of etiologies and symptomatologies of acute allergic reaction, this study's purpose was to define both variables in a cohort of consecutive patient encounters in a large, urban EMS system in the United States. Methods: Retrospective chart review of consecutive encounters from January 1, 2012 until January 1, 2013 in the study EMS system. Patient cohort was selected by chief complaint documentation as "allergic reaction" or "insect sting". Descriptive analysis was performed on history of presenting complaint and physical assessment comments. Results: In the 196 patients, etiology was noted as follows: idiopathic $71(36.2 \%)$; medication unspecified class $30(15.3 \%)$; medication antibiotic class $26(13.3 \%)$; insect sting 16 (8.2\%); food nut class 12 (6.1\%); food unspecified class 10 $(5.1 \%)$. The remaining 31 patients were allocated among 8 other classifications. None represented more than $3.6 \%$ of cohort. Symptomatology was noted as follows: urticaria 96 (49.0\%); dyspnea $72(36.7 \%)$; edema either generalized or localized site 41 (20.9\%); wheezing 35 (17.9\%); edema of airway 29 (14.8\%); erythema 24 (12.2\%); altered mental status $13(6.6 \%)$. Of the remaining symptoms, none represented 
more than $5.0 \%$ of cohort. Conclusion: Acute allergic reactions treated in a large, urban EMS system are most commonly idiopathic in etiology. Symptoms of acute allergy are mutliple, most frequently presenting with uticaria, dyspnea, and/or edema.

Keywords: emergency medical services, acute allergic reactions, signs and symptoms

\section{P043}

Impact of a regional public wait times website on regional ED volumes - a preliminary study

E. Grafstein, MD, M. de Moor, BSc, MA, M. Stewart, R. Stenstrom, MD, PhD, C. Startup, MA, Department of Emergency Medicine, University of British Columbia, Vancouver, BC

Introduction: Little research exists on the manipulation of patient inputs to EDs. Websites that display ED wait times may be a way to lessen the burden on busier EDs. Methods: A public ED wait time website involving all 5 of the Vancouver Coastal Health - Providence Health Care regional urban EDs went live in April 2013. 3 of these EDs, Vancouver General Hospital (VGH), St. Paul's Hospital (SPH), and Mount St. Joseph Hospital (MSJ) are $<4.5 \mathrm{~km}$ apart. The other two EDs are about 11 and $18 \mathrm{~km}$ away across a major bridge. The website (www.edwaittimes.ca) displays both average and 90\%ile time to MD. Website hits are tracked through Google Analytics (Google, Mountain View, CA). A proxy for access block occurs when total patients in the ED reach the 99\%ile value based on fiscal 2012-13 data. We built a tool (Tableau Software, Seattle, WA) that tracks when a site reaches this $99 \%$ ile value. Data was compared by fiscal year for periods 1-9 from 2010-11 onward. ED administrative data is accumulated daily and stored in a central data repository. This data has been previously validated for reliability and accuracy. We reviewed specific ED volumes, ambulance traffic, acuity level, and time to physician. Results: There were 220,508 visits made by 147,557 patients post dashboard go-live. Website hits during this period were 83,156 made by 41,919 people. Regional average volume increase year over year was 8,294 patients/year (4.1\%). Regional volume increase after the website came online was not significantly greater than in other years (8437 patients, $4.0 \%$ ). There was a significant increase in patient volume compared to previous years at the MSJ site of 1920 patients, $12.1 \%$ increase; $(\mathrm{p}<0.01)$. At this site wait times to MD were $38 \mathrm{~min}$ (avg.) and $76 \mathrm{~min}(90 \%$ ile). These are the shortest regional average and $90 \%$ ile times to MD. For VGH these time metrics were 59 and 129 minutes respectively. The two Vancouver EDs (VGH and SPH) in proximity to MSJ saw an increase in patient volume that was less than in the previous two interval years (1200 patients, $2.3 \%$ increase and $1450,2.5 \%$ increase respectively; $p$ $<0.01)$. In spite of increasing volumes, MSJ saw only 1 day with $99 \%$ ile red surge alerts for total patients in the ED. VGH and SPH saw 46 and 39 days with a surge alert for total patient in the ED. Monthly surges trended downward at VGH. Conclusion: There is modest evidence that public websites can deflect patients away from more congested EDs. There is no evidence that this tool increased overall regional ED usage.

Keywords: public website, ED wait times, overcrowding

\section{P044}

Which test leads to the shorter ED length of stay for patients with appendicitis: ultrasound or CT scan?

S.H. Gray, MD, R. Simard, MD, J.S. Lee, MD, MSc

St. Michael's Hospital, Toronto, ON

Introduction: The diagnosis of appendicitis in the Emergency Department (ED) often involves computed tomography (CT) or ultrasound.
However, these modalities differ with respect to accuracy, availability, radiation dose and timing. The goal of this study was to determine if ED length of stay (LOS) was shorter if CT was used as the initial imaging test for adult patients with suspected appendicitis. Methods: A retrospective chart review was performed at two academic urban hospitals from January 2007 to August 2010. A single trained abstractor collected data onto a standardized data abstraction template. All adult ED patients with appendicitis diagnosed on CT or ultrasound were included. Outcome variables were defined a priori including imaging modalities used, ED length of stay, hospital length of stay and survival. Multivariate regression was used to determine predictors of ED LOS. ED physicians were surveyed regarding their current imaging practice and preferences for diagnosing appendicitis. Results: 765 appendicitis patients were included with 479 (63\%) having CT only, 192 (25\%) having ultrasound (US) only, 88 (11\%) having US then CT, and $6(1 \%)$ having CT then ultrasound. CT only or CT first had a mean LOS of $10.2 \mathrm{~h}$ (95\%CI 9.7-10.6) compared to ultrasound only or US first leading to a mean LOS of $10.4 \mathrm{~h}$ (95\%CI 9.8-11). Patient who received only an ultrasound had the shortest ED LOS $(8.9 \mathrm{~h}, 95 \% \mathrm{CI}$ 8.3-9.5), however if patients had an US then a CT the mean LOS was 13h. (95\%CI 12.6-14.8). Multivariate regression confirmed that CT first was associated with a longer LOS when controlling for whether patients received both ultrasound and CT scan. 18 out of 34 physicians surveyed responded (53\%). $72 \%$ of ED physicians surveyed responded that ordering a CT scan first would result in the shorter ED LOS. Conclusion: In contrast to the perception of our ED physicians, using CT as the first imaging test did not lead to shorter ED LOS for patients with appendicitis. Ultrasound, if diagnostic, led to the shortest ED LOS. However, if patients needed a CT after their ultrasound, their ED LOS was prolonged. Further research should identify patients more likely to have an indeterminate ultrasound and the impact of ultrasound availability on ED LOS.

Keywords: appendicitis, length of stay, imaging

P045

A characterization of trauma patients who have undergone procedural sedation

R. Green, MD, M. Butler, BSc, Dalhousie University, Halifax, NS

Introduction: Multisystem patients often require invasive procedures, such as chest tube insertion and fracture management as part of their trauma care. To provide optimal patient comfort, procedural sedation (PSA) is utilized. However, patients with multisystem traumatic injuries may be physiologically challenged as opposed to the usual PSA population. Therefore, the incidence of adverse events (AEs) may be increased in this population. This study characterizes and describes the incidence of AEs in multisystem patients who received PSA under the direction of a paramedic. Methods: This is a retrospective analysis of 101 patients that were trauma team activations at the Halifax Infirmary (HI) from January 2006 to December 2012. Data were collated from the Nova Scotia Trauma Registry and the HI PSA registry. Results: The majority trauma patients that underwent PSA were male (75.3\%), and most patients were relatively healthy (ASA I, 78.2\%). The majority of PSAs were orthopedic manipulations (84.2\%), and almost all patients were admitted to a high intensity in-hospital unit (ICU 9.9\%, IMCU 61.4\%). Adverse events were common (45.4\%), with hypotension $(19.8 \%)$ and tachypnea $(22.8 \%)$ the most common. Two patients died in-hospital, and those who survived, the average LOS was 9.64 days. Conclusion: AEs in PSA are common in the multisystem trauma patient. This population represents a unique subset of the PSA population which requires additional research to optimize patient outcomes.

Keywords: trauma, procedural sedation 
P046

The impact of normal saline versus ringers lactate on patient outcomes

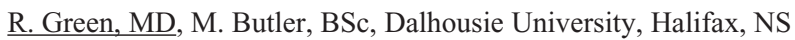

Introduction: Crystalloid solutions are given to the majority of patients who undergo endotracheal intubation (ETI). The goal of this study is to determine if an association exists between the administration of normal saline and/or ringers lactate in patients undergoing ETI and adverse patient outcomes. Methods: A structured chart audit was performed of adult patients intubated for a vascular surgery procedure at the QEII over a 3-year period. Patients were identified and data collated from multiple electronic databases. Data not available electronically was abstracted from the patient chart by a trained co-investigator. Patients were separated into three groups: those who received only normal saline $(\mathrm{n}=158)$, those who received only ringers $(\mathrm{n}=419)$ and those who received both $(n=207)$. Patients who received saline were considered the baseline group. To determine the association of crystalloid solutions with adverse patient outcomes we recorded in-hospital mortality, vasopressor days, inotropes days, hemodialysis days and length of ICU stay, and controlled for confounding factors such as initial patient co-morbidity and urgency of procedure. To evaluate overall patient outcome, we assessed a composite end-point of in-hospital mortality, ventilator days, inotrope days, and length of ICU stay using a multivariate analysis. Results: Overall, 784 patients received only crystalloid solutions. The groups were similar in age $(\mathrm{P}=0.141)$. Overall, the groups were different in gender distribution $(\mathrm{P}=0.0005)$ and overall health ( $\mathrm{ASA} \geq 2 ; \mathrm{P}=0.0027$ ). When controlling for confounding factors, receiving ringers lactate fluid was a significant predictor for decreased ICU admission $(\mathrm{OR}=0.27, \mathrm{P}<0.0001, \mathrm{CI}=\{0.16,0.46\})$, post-op vasopressor requirements $(\mathrm{OR}=0.39, \mathrm{P}=0.014, \mathrm{CI}=\{0.18$, $0.82\})$, post-op ventilator requirements $(\mathrm{OR}=0.24, \mathrm{P}<0.0001$, $\mathrm{CI}=\{0.13,0.41\})$, hemodialysis requirement $(\mathrm{OR}=0.04, \mathrm{P}=0.034$, $\mathrm{CI}=\{0.0006,0.47\})$ and the composite end-point $(\mathrm{OR}=0.30, \mathrm{P}<0.0001$, $\mathrm{CI}=\{0.18,0.49\})$. The saline-ringers combination remained associated with an increase in ICU admission $(\mathrm{OR}=2.05, \mathrm{P}=0.005, \mathrm{CI}=\{1.24$, $3.43\})$ and the composite end-point $(\mathrm{OR}=2.07, \mathrm{P}=0.005, \mathrm{CI}=\{1.25$, $3.44\}$ ). Conclusion: Ringers lactate was given to the majority of patients requiring endotracheal intubation and was associated with a decrease in adverse patient outcomes compared to $0.9 \%$ saline. Further research is required to elucidate the effect of crystalloid solution choice on patient outcomes.

Keywords: crystalloid

P047

The impact of peri-intubation physiologic adverse events on longterm patient outcomes

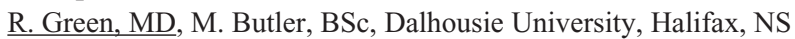

Introduction: Endotracheal intubation (ETI ) is often a necessary procedure in the critically ill patient. Few studies have assessed the incidence of physiologic adverse events (AEs) secondary to ETI. The goal of this study is to evaluate the incidence of physiologic adverse events within a vascular surgery population during ETI. Methods: A structured chart audit was performed of adult patients intubated for a vascular surgery procedure at a quaternary centre over a 3-year period. Patients were identified and data collated from multiple electronic databases. Data not available electronically was abstracted from the patient chart by a trained co-investigator. Adverse events in the periintubation period (within 15 minutes of ETI) included: - Hypoxia $(\mathrm{SaO} 2<80 \%)$ - Hypertension ( $\mathrm{SBP}>180 \mathrm{mmHg}$, or MAP $>110 \mathrm{mmHg}$ ) - Tachypnea ( $>30$ respirations per min) and bradypnea $(<10$ respirations per min) - Tachycardia ( $>120$ beats per minute) and bradycardia $(<50 \mathrm{bpm})$ - The rate of occurrence of post-intubation hemodynamic instability, defined as a decrease in systolic blood pressure (SBP) to $<80 \mathrm{mmHg}$, or a decrease in SBP of $>20 \%$ from baseline, or a decrease in mean arterial pressure to $<50 \mathrm{~mm} \mathrm{Hg}$, or the initiation of any vasopressor medication at any time in the 15 minutes following intubation. We also evaluated if having an increasing number of physiologic adverse events was associated with an increased incidence of poor patient outcomes such as: - In-hospital mortality - Post-op hemodialysis requirements - Post-op ventilator requirement - Post-op vasopressor requirement - Requirement for ICU admission To account for overall patient outcome, we also assessed a composite end-point of any the above events occurring. Results: Overall, 1395 patients required intubation between 2007 and 2009. The mean age of the patients was 67.4 years and $63.7 \%$ of patients were male (888/1395). $67.03 \%$ of patients were generally unhealthy, being assessed as an ASA level of 3 or higher. The most common AE was hypertension (61.6\%), and least common was bradypnea (5.52\%). Having an increase in the number of adverse events was associated with an increase in the probability of poor patient outcome. Each additional AE increased the probability of in-hospital mortality by $42 \%$, and an increase of $72 \%$ for the composite endpoint. Conclusion: We observed a high rate of peri-intubation physiologic adverse events. Incurring an increased number of AEs is associated with an increase in poor patient outcome. Further investigation is warranted in evaluating associations between AEs during endotracheal intubation and patient outcomes.

Keywords: airway management

P048

The incidence and factors associated with the development of postintubation hemodynamic instability

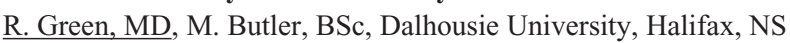

Introduction: Post-intubation hemodynamic instability (PIHI) is a common adverse event that has been associated with poor patient outcomes in emergency department endotracheal intubations. The objectives of this study were to determine factors that influence the development of PIHI. Methods: A structured chart audit was performed of adult patients intubated for a vascular surgery procedure at the QEII over a 3-year period. Patients were identified and data collated from multiple electronic databases. Data not available electronically was abstracted from the patient chart by a trained co-investigator. PIHI was defined as a decrease in systolic blood pressure (SBP) to $<80 \mathrm{mmHg}$, or a decrease in SBP of $>20 \%$ from baseline, or a decrease in mean arterial pressure to $<50 \mathrm{~mm} \mathrm{Hg}$, or the initiation of any vasopressor medication at any time in the 15 minutes following intubation. To determine factors that led to PIHI we assessed the effect of co-morbidity status (e.g. diabetes, hypertension), the urgency of the procedure, the time of day and the amount of fluid and types of medications given to a patient before intubation. Results: Overall, the incidence of PIHI was $60 \%(837 / 1395)$ in the 1395 patients identified from 2007-2009. There was no difference in age, gender, or intravenous fluid administered between the PIHI-group to those who did not develop PIHI. Patients who developed PIHI were more likely to receive fentanyl, 
remifentanil, or sufentanil, have had a recent MI ( $<12$ months), or had a history of COPD, diabetes and anemia $(\mathrm{Hg}<100)$. On multivariate analysis, factors associated with an increase in the incidence of PIHI were fentanyl dose $(\mathrm{OR}=1.01 ; \mathrm{CI}=\{1.01,1.02\} ; \mathrm{P}<0.001)$, ketamine dose $(\mathrm{OR}=1.04 ; \mathrm{CI}=\{1.02,1.07\} ; \mathrm{P}=0.001)$, propofol dose $(\mathrm{OR}=1.01$; $\mathrm{CI}=\{1.00,1.01\} ; \mathrm{P}<0.001)$, remifentanil dose $(\mathrm{OR}=1.01 ; \mathrm{CI}=\{1.00$, $1.02\} ; \mathrm{P}=0.002)$, rocuronium dose $(\mathrm{OR}=1.02 ; \mathrm{CI}=\{1.01,1.03\}$; $\mathrm{P}<0.001)$, sufentanil dose $(\mathrm{OR}=1.13 ; \mathrm{CI}=\{1.09,1.18\} ; \mathrm{P}<0.001)$, receiving a HES solution $(\mathrm{OR}=4.37 ; \mathrm{CI}=\{2.35,8.71\}, \mathrm{P}<0.0001)$, needing an $\mathrm{OR}$ within 24 hours $(\mathrm{OR}=1.91 ; \mathrm{CI}=\{1.21,3.01\} ; \mathrm{P}=0.005)$, anemia $(\mathrm{OR}=1.49 ; \mathrm{CI}=\{1.02,2.18\}, \mathrm{P}=.049)$ and $\mathrm{COPD}(\mathrm{OR}=1.45$, $\mathrm{CI}=\{1.03,2.06\}, \mathrm{P}=0.046)$. Conclusion: $\mathrm{PIHI}$ occurs in $60 \%$ of vascular surgery patients. Factors associated with an increased incidence of PIHI are urgency of procedure, COPD, anemia and increasing doses of fentanyl, ketamine, propofol, rocuronium and sufentanil.

Keywords: post-intubation hemodynamic instability

\section{P049}

CAEP Stroke Practice Committee Position Statement on thrombolysis for acute ischemic stroke

D.R. Harris, MD, MHSc, E. Lang, MD, Department of Emergency Medicine, St. Paul's Hospital, Vancouver, BC

Introduction: The management of acute ischemic stroke in emergency departments, by emergency physicians, is time sensitive and complex. Recognizing the need to provide recommendations for emergency physicians throughout Canada, the position statement for thombolysis for acute ischemic stroke needed to be updated and expanded upon the original position statement from 2001. The objective of the CAEP Stroke Practice Committee was to review the current literature and published guidelines for the management of acute ischemic stroke patients, including thrombolysis, and provide recommendations. Methods: This committee was composed of 19 emergency physicians, from various practice settings, across Canada, who initially met through teleconference in September 2012. The strength of recommendations and the quality of evidence designations reflect a consensus-based process: although not adhering strictly to the GRADE method of consensus, this methodology was followed as a template. An initial literature review was performed; literature was disseminated to committee members. Reviews were performed from September 2012 to May 2013, through online means and teleconferences. An in-person meeting was held in June 2013 at the CAEP Annual Scientific Meeting in Vancouver, to review recommendations and finalize the document. The Committee heavily relied upon the Canadian Best Practices for Stroke Care for the literature review and evidence. Key topics of acute ischemic stroke management identified by the Committee were: Stroke Systems of Care; Emergency Medical Services (EMS) Considerations; Thrombolytic Therapy within 3 Hours of Stroke Symptom Onset; Thrombolytic Therapy within 3 to 4.5 Hours of Stroke Symptom Onset; Optimal Post Thrombolysis Monitoring / Transitions in Care, and; Telestroke. Results: Of the six topic areas, 22 recommendations were agreed upon. In summary: stroke systems of care should be developed to optimize prehospital triage and transport to designated stroke centers; intravenous thrombolysis for acute ischemic stroke patients should be offered to eligible patients who present within three hours of stroke symptom onset; intravenous thrombolysis for eligible patients who present between 3 to 4.5 hours from stroke symptom onset should not routinely be offered intravenous thrombolysis, except in specialized centers; the majority of admitted stroke patients should be managed on inpatient stroke units, and; telestroke should be supported as a means to provide acute stroke expertise where local support is limited. Another key topic area was identified, Rural Stroke Care, which is under review. Conclusion: The CAEP Stroke Practice Committee Position Statement on Thrombolysis in Acute Ischemic Stroke provides 22 recommendations for emergency physicians in managing acute stroke in the ED.

Keywords: stroke, guideline

P050

Evaluation of a brief on-line teaching module training emergency physicians and residents how to interpret hydronephrosis and its gradations using point of care ultrasonography

B. Hassani, MD, S.L. McLeod, MSc, A. Shah, MD, M. Mann, MD, R. Arntfield, MD, University of Western Ontario, London, ON

Introduction: Many Canadian emergency physicians (EPs) are trained in the core indications for point-of-care ultrasound (POC-US). Achieving training in more "advanced" applications, including detection of hydronephrosis, is currently complicated by a shortage of training opportunities. The objective of this study was to evaluate the influence of a brief, online educational tutorial on an EP's ability to accurately diagnose and grade hydronephrosis. Methods: Academic and community EPs and residents were invited to participate in this online study. Following a pre-test of interpreting US images of varying degrees of hydronephrosis, participants viewed a 10-minute online tutorial describing how to diagnose and grade hydronephrosis and recognize possible mimics on POC-US. Acting as their own control, participants then completed a post-test comprised of similar US images. US images were from ED patients with a clinical presentation of renal colic and diagnostic US reports that were categorized into 4 categories: none, mild, moderate, and severe hydronephrosis. Results: The response rate was $74.0 \%$ (74/100). 69 (93.2\%) respondents had completed an introductory POC-US course with $22(29.7 \%)$ having completed an advanced application course. 59 (79.7\%) stated that they were "not at all comfortable" diagnosing and grading hydronephrosis using POCUS. There was a significant increase from the pre-test (38.9\%) to posttest $(73.3 \%)$ in correct identification of hydronephrosis and its gradation ( $34.4 \%$; 95\% CI: $29.8 \%, 38.9 \%$ ). When the grades of hydronephrosis were removed, there was a $15.9 \%(95 \%$ CI: $12.6 \%$, $19.2 \%)$ increase from the pre-test $(58.7 \%)$ to post-test $(72.7 \%)$ in correct identification of hydronephrosis. There was no difference between physicians that had taken a basic versus an "advanced" application US course, institution, level of training or age group. Physicians practicing emergency medicine for less than 5 years did significantly better than physicians practicing $15+$ years $(\mathrm{p}=0.03)$. Conclusion: This study demonstrates that an on-line educational module substantially improves the accuracy of EP interpretation of hydronephrosis as well as its gradations. This brief online tutorial is an effective and accessible tool and could be utilized as one component of an advanced POC-US training curriculum.

Keywords: point-of-care ultrasound, renal colic

P051

Team-based errors in trauma resuscitation: a systematic review H. Heipel, MD, M. McGowan, MHK, C. Hicks, MD, MEd, University of Toronto, Toronto, ON 
Introduction: Management of multi-system trauma is fraught with challenges owing to complex clinical presentations, time dependency for intervention and adhoc assembly of trauma teams. Despite ATLS and dedicated trauma centers, team-based medical error occurs in the majority of trauma resuscitation. This systematic review attempts to determine the nature and types of team-based errors occurring in the trauma bay. Methods: A primary search of Medline, EMBASE, Web of Science, and Cochrane Databases for studies after 1980 (ATLS implementation) was conducted with keywords (human factors, wounds and injuries, medical errors, trauma centers, emergency medicine) and augmented by a bibliographic reference search and Google scholar to increase sensitivity and rigor. Non-English articles, case studies, editorials reviews, and letters were excluded. Two reviewers independently screened titles and abstracts, assessed quality and suitability of the studies by full article review, with level of agreement, determined by kappa, assessed at each level. Disagreement was resolved by consensus, including an independent third reviewer. Selected studies were qualitatively analyzed. Results: Primary search identified 893 citations; 17 by secondary hand search. 27 abstracts were evaluated (kappa 0.65) and 11 articles (kappa 0.6) selected for text review with 2 fulfilling inclusion criteria. The first paper was a video registration incorporating 1256 patients and 387 team performance analysis over 2 years. Error was defined by ATLS deviation. Team organization and communication errors were quantitatively and qualitatively analyzed using a standardized scoring form. Study found a significant reduction of errors with adequate pre-hospital report, effective leadership, efficient leadership, evident leader, and team organization $(p<0.05)$. The second paper included a chart review of 764 patients over 5 years using JCAHO error taxonomy to analyze team errors. These authors found that human errors predominated contributing to preventable or potentially preventable deaths. The paper concluded that the majority of preventable errors were because of the complex trauma team incorporating many specialties and level of experience. However, they did not break down the specific human errors leading to death. Conclusion: Technical and systematic error is well reported in the trauma literature; however, there remains a dearth of understanding in the contribution of human factors as a source of error in trauma resuscitation. Team-based errors in trauma resuscitation are poorly documented in the literature and patient records, yet this does not preclude its existence or importance in enhancing patient care and safety.

Keywords: patient safety, trauma, team error

\section{P052}

Sustained knowledge acquisition among Rwandan physicians participating in a six-month ultrasound training program

P.C. Henwood, MD, E.R. Douglass, BA, J.S. Rempell, MD, MPH, A.F. Murray, MD, A.S. Liteplo, MD, D.C. Mackenzie, MDCM, M.M. Leo, MD, A.J. Dean, MD, S. Vaillancourt, MD, MPH, E. Krebs, MD, D. Dukundane, MD, S. Rulisa, MD, V. Noble, MD, Brigham and Women's Hospital, Boston, ON

Introduction: Point-of-care ultrasound (POCUS) is ideal for diagnostic use in resource-limited environments, but its use is often limited by lack of local expertise. Some training courses have been established to bridge this knowledge gap, but little is known about the efficacy of these courses. This study evaluated the effectiveness and sustainability of a six-month POCUS training program for
Rwandan physicians. Methods: A prospective observational cohort study of Rwandan physicians was conducted over a six-months. Participants completed a ten-day course introducing ultrasound skills, followed by a six-month clinical phase. Trainees used ultrasound independently with remote image review, completed regular observed structured clinical examinations (OSCE) in POCUS, and received ongoing feedback and web-based mentorship. An imagebased assessment (IBA) was administered before and after the tenday course, and again after six months. Hands-on image acquisition skills were assessed via OSCEs administered at four time points. All calculations were by paired t-test. Results: Seventeen Rwandan physicians completed the initial course. All completed the IBA prior to and at the completion of the training phase. Seven trainees were lost to follow up at the six-month mark due to reassignment. Ten trainees completed the six-month IBA. Mean scores on the IBA increased from $36.9 \%$ to $74.3 \%$ after the training phase $(\mathrm{p}=<.001)$ and to $85 \%(\mathrm{p}=0.04)$ at the end of the clinical phase. Ten trainees completed at least three OSCEs. The average score on the initial OSCE was $81.7 \%$. Of ten trainees who completed at least three OSCEs, scores were preserved (mean 82.9\%). Conclusion: Trainees demonstrated significant knowledge improvement after an intensive introductory ultrasound course, which increased through the training program. Mean OSCE scores remained above $80 \%$ throughout the course. Participants in an ultrasound training program with an initial training phase and periodic skill reinforcement can acquire and retain ultrasound knowledge and scanning skills.

Keywords: ultrasound, global health, education

\section{P054}

Impact of a new ED on regional ED and urgent care utilization and wait times (Build it and they will come)

G. Innes, MD, A. McRae, MD, D. Wang, MSc, E. Lang, MD, University of Calgary, Calgary, $\mathrm{AB}$

Introduction: In Jan 2013, Calgary added a fifth hospital and emergency department, the South Health Campus (SHC), to our system of 3 adult EDs, one pediatric ED (ACH) and 2 urgent care centres (UCC) servicing a population of 1.2 million. Our objective is to describe its impact on regional ED utilization and waiting times. Methods: Calgary has common ED processes, information collection and IT systems at all ED and UCC sites. This was a descriptive analysis of administrative data for a 9-month period before and after SHC opening. Data were annualized to provide a simulated one-year summary of ED utilization and operational performance. Results: In 2012, prior to opening SHC, Calgary had 319,474 ED visits, including 250,000 to adult sites, 69,474 to ACH, and 106,682 to UCCs. In 2013, the new ED saw 58,735 visits. This was offset by 6456 fewer adult ED visits $(-2.6 \%)$ and 6593 fewer UCC visits $(-6.2 \%)$, but 2373 more ACH visits $(+3.4 \%)$. The new ED saw 36,968 CTAS 1-3 visits (63\%). This was associated with 27,724 fewer CTAS 1-3 visits at the other adult EDs (a $13.4 \%$ drop), 1441 fewer CTAS $1-3$ visits to UCCs (an 11\% reduction), and 2139 more $\mathrm{ACH}$ visits (a 5.5\% increase). Redistribution of high acuity patients caused the overall CTAS 1-3 proportion at adult sites to fall from $83 \%$ to $74 \%$ (three-site average), but did not change UCC or ACH acuity mix. Volume and acuity changes were most marked at sites closest to the new hospital. Median CTAS 3 wait times fell by $12.1 \%$ (109 to 96 minutes) at adult EDs and by 26\% (115 to 85 minutes) at UCCs, but were unchanged at $\mathrm{ACH}$ (64.4 minutes). ED 
LOS for discharged patients fell from 3.8 to 3.6 hours at adult sites and from 1.9 to 1.4 hours at UCCs, but remained constant at ACH (2.3 hours). Admitted patient LOS fell from 9 hours to 8.4 hours at adult EDs and rose slightly from 6.0 to 6.3 hours at $\mathrm{ACH}$. Conclusion: SHC opening was associated with a $17 \%$ increase in total regional ED visits $(\mathrm{N}=54,652)$ and a $6.2 \%$ drop in UCC visits. The rise in ED volume rise cannot be attributed solely to the new ED, since regional ED volumes had been rising $\sim 10 \%$ annually for the previous three years. Patient redistribution led to a lower acuity mix at existing adult EDs. The addition of a new ED relieved some volume and acuity stress at other sites, but also led to a larger than expected increase in regional ED volumes, suggesting that increased ED capacity facilitates convenience care as well as emergency care.

Keywords: emergency services, utilization, access

\section{P055}

Region-wide variability of operational performance and utilization behaviour for emergency physicians

G. Innes, MD, E. Lang, MD, D. Wang, MSc, A. McRae, MD, University of Calgary, Calgary, $\mathrm{AB}$

Introduction: The ability of an emergency department to manage growing patient volumes depends heavily on two key bottlenecks: emergency physician (EP) capacity and stretcher availability. Assuming appropriate staffing levels, the main determinant of physician capacity is number of patients seen per hour. Stretcher availability depends largely on the number of patients admitted and boarded, as well as physician utilization behaviour (i.e. how efficiently EPs use stretcher time). Our objective was to describe EP operational performance characteristics and variability in a large population-based sample. Methods: All EPs in Calgary belong to an integrated city-wide department that provides emergency care (on a fee-for-service basis) for the region. We electronically capture MD performance parameters for inclusion in individual MD report cards. Parameters include patients seen per-hour, admission rate for CTAS 3 patients, MD throughput time from sign-up to patient discharge, 72-hour bounce-back requiring hospitalization, and imaging utilization. This study is a descriptive analysis of the performance of all $141 \mathrm{ED}$ physicians who treated more than 300 patients in 2012. Results: In 2012, 108 male and 33 female EPs treated 250,000 ED patients (83\% CTAS 1-3) at 3 urban hospitals. Mean EP age was 44.2 years (SD, 9.4) with a range of $29-70$. Sixty physicians $(45.4 \%)$ are FRCP certified, and the remainder CCFP-EM. Excluding end-ofshift handovers, physicians saw a median of 2.02 new patients per hour (IQR, 1.83-2.27), and 90\% of EPs were between 1.57 and 2.71 patientsper-hour. Median admission rate for CTAS 3 patients was $16.3 \%$ (IQR, 14.6-18.3), and $90 \%$ were between $10.3 \%$ and $22.5 \%$. Median processing time (sign-up to discharge) for CTAS 3 patients was 1.75 hours (IQR, 1.45-1.98), with $90 \%$ between 0.98 and 2.57 hours. Median rate of bounce-back requiring hospitalization was $1.06 \%$ (IQR, 0.8-1.25), with $90 \%$ between $0.46 \%$ and $1.7 \%$. The median physician ordered an ultrasound for $4.9 \%$ of patients (IQR, 3.7-6.1), with $90 \%$ between 2.4 and $8.7 \%$. The median physician ordered a CT for $14.1 \%$ of patients (IQR, 11.5-16.3), with $90 \%$ between 7.5 and $21.2 \%$. Conclusion: EP productivity and utilization behaviour varies substantially enough to impact departmental capacity and operations. Future studies should address determinants of variability and strategies to reduce variability where this can be safely accomplished.

Keywords: emergency services, efficiency

\section{P056}

Can a focused education program increase staff familiarity with an emergency department disaster plan?

C. Jarvis, MSc MD, QEII Health Sciences Centre, Halifax, NS

Introduction: The effectiveness of Emergency Department (ED) disaster plans depends on a number of factors, including staff awareness of the existence of the plan as well as how to access and use it. Previous informal inquiries suggested that many of the ED staff lacked this basic awareness. The objective of this study was to measure the impact of education on perceived disaster preparedness and level of awareness of the ED disaster plan. Methods: A series of educational events were offered over a 6-month period, including a live exercise, small group teaching, and informational posters throughout the ED. An online survey was developed to assess the perceived preparedness of both the respondent and the Emergency Department (ED) as a whole to respond to both mass casualty (MCI) and chemical, biological, radiation, nuclear and explosion (CBRNE) incidents. A Likert scale was used for subjective questions, with 1 being least prepared and 5 most prepared. Means were calculated and compared using the Wilcoxon test. Awareness of the disaster plan was also explored. All ED staff were emailed invitations to participate in the survey prior to the educational events, and again after the conclusion of the educational events. Results: Fifty-five of 232 staff ( $23 \%$ ) responded to the pre-education survey, and $25.5 \%(53 / 208)$ to the post-survey. Mean perceived preparedness of respondents for MCI disasters increased from $2.98(+/-1.10)$ before education to $3.29(+/-0.92$, NS) after, and for CBRNE disasters, 2.33 $(+/-1.09)$ versus $2.92(+/-1.06, p=0.006)$. Awareness of disaster plan existence increased from $80.3 \%(\mathrm{n}=51$ respondents $)$ to $94.1 \%$ $(\mathrm{n}=51)$.Knowledge of how to access the plan increased from $77.5 \%$ $(n=40)$ to $95.8 \%(n=45)$. Before education, $47.5 \%(19 / 40)$ of respondents felt the disaster plan was useful compared to $75.0 \%$ (36/48) after. Finally, basic knowledge increased from $26.5 \%$ (13/49) before education to $55.6 \%$ (25/45). Conclusion: Focused educational efforts improved all scores in an online survey regarding perceived disaster preparedness and awareness of the disaster plan among ED staff. These improved scores support efforts around disaster plan education.

Keywords: disaster, teaching, preparedness

\section{P057}

Assessing attitudes toward inner-city populations: what tools are available?

M. Jepson, BSc, A. Kirkham, BA, MD, K.E. Smith, BSc, MD, G. Salvalaggio, MSc, MD, K. Dong, MD, MSc, University of Alberta, Edmonton, $\mathrm{AB}$

Innovation concept: The 'inner city' includes many diverse patient groups whose medical care can be complicated by provider biases in the emergency department. Medical learners often feel uncomfortable working with these patients, and attitudes toward inner city populations actually worsen over the course of medical education. In order to improve how we teach learners to care for vulnerable patient groups, we must have tools to evaluate learner competency. One component of evaluation includes the use of a validated attitudinal assessment tool. Research and educational initiatives have traditionally focused on specific subsets of the inner city population (e.g. patients who use intravenous drugs, are homeless, have HIV or are involved in the survival sex trade), but these often do not occur in isolation. When evaluating 
learner competency to work with this population, an attitudinal assessment tool should be able to evaluate all subsets of the inner city population. Methods: A librarian-facilitated literature search was completed to identify articles that assessed healthcare professional attitudes toward different subsets of the inner city population including those individuals with the following characteristics: homeless or marginally housed, street involved, survival sex trade involved, use of intravenous drugs, drug or alcohol misuse, HIV/AIDS, and prison populations. Results were screened, and identified attitudinal assessment tools used were retrieved, summarized and categorized. Curriculum, tool, or material: Our search found 597 articles, 173 remained after initial screen and 90 articles using attitudinal assessments were identified. Tools focused on homeless (5), substance using (45), mental health (6), prison (7), HIV/AIDS (10), and other/multiple (17) populations. No tools were identified that examined attitudes towards inner city populations as a whole. Conclusion: Our review found that a validated and generalizable attitudinal assessment tool that examines health care learner biases toward inner-city groups as a whole is currently not available. Existing tools reflect a fragmented approach to evaluating learner attitudes towards inner city patients. A generalizable and practical tool is needed for use by medical educators working to improve the quality of care provided to these marginalized patients.

Keywords: innovations in EM education, attitudes, inner City

\section{P058}

Incorporating the patient experience with an early acute pain management quality imitative: development and preliminary findings of an in situ survey

J. Jolley, BSc, M. McGowan, MHK, K. Nixon, MSc, K. Gaunt, MSc, L. Barratt, MSc, D. MacKinnon, MD, E. Nagatu, G. Martin, MSc, G. Bandiera, MD, MEd, J. Spence, MD MSc, St. Michael's Hospital, Toronto, ON

Introduction: Oligoanalgesia is a common source of patient dissatisfaction in the ED. While the NRC Picker is validated to measure ED patient satisfaction including pain management, its exclusion of disadvantaged groups (mental health, homeless, low literacy and non-English speaking patients) limits its interpretation within an inner city population. We sought to develop an in situ survey to measure the patient experience in parallel with a quality improvement protocol for early acute pain. Methods: An inter-professional team developed a survey instrument in multiple stages: i) content was derived by thematic analysis of a multidisciplinary focus group; (ii) survey design encompassed a blend of Likert scale and open-ended questions; (iii) survey was piloted among ED patients to refine usability, comprehension and content with enhancements made prior to full deployment. Across convenience sampling of weekdays and ED shifts, all patients triaged to a rapid assessment zone (RAZ) in an inner city ED with pain were eligible and provided with the 10-question survey. Results: Over 4-weeks, 108 surveys were completed with the majority $(69 \%)$ reporting severe pain at triage $(82 \%)$. There was perceived minimal delay (48\%) until staff (MD 36\%, RN 35\%, both 11\%) discussed pain relief, most often through medication (83\%). Patients provided mixed responses regarding the adequacy of therapy and relief (yes $46 \%$; no $32 \%$ ); however, most felt ED staff were doing everything they could ( $74 \%$ definitely) with $42 \%$ satisfied (29\% very) with pain relief in the ED. Conclusion: Quality patient care includes early, effective pain management; incorporating the patient voice is an important quality dimension to augment time-based metrics and provide ongoing feedback to front-line staff.
Keywords: patient satisfaction, quality improvement, pain management

P059

The use of electronic health records for emergency department quality improvement evaluations in Calgary

S. Jones, MD, MHSc, J. Bastian, BSc, MD, D. Wang, MSc, A. McRae, $\mathrm{MD}$, E. Lang, MD, University of Calgary, Calgary, $\mathrm{AB}$

Innovation concept: Recently Calgary area emergency departments digitized results from each patient encounter into a database of electronic health records. Utilizing electronic health records enables us to quickly complete administrative data enquiries, which gives us a platform to easily access, organize and interpret comprehensive retrospective data. In doing so, we have established a practical tool to conduct quality improvement evaluations directly related to the practice of emergency medicine in Calgary. Methods: After identifying an area of interest, a study protocol is developed and data recovery from electronic health records can commence. Our studies fall under quality improvement, which enables us to complete the studies without ethics approval. Once the data has been collected, retrospective conclusions about our study question can be made and knowledge translation to our Calgary emergency department staff can begin. Curriculum, tool, or material: Over the last year we have compiled results looking at gender specific differences in anaphylaxis, inter-observer variability in CTAS scoring, emergency department presentations of hereditary angioedema, and delayed diagnosis of intracranial hemorrhage after head trauma. The key to our data is the ability to quickly establish trends in current Calgary emergency department practice, which can be immediately used to educate our emergency department staff and improve patient care. Knowledge translation has been completed through educational rounds, direct physician feedback, research days and presentations at Canadian and International conferences. Our findings have led to changes to order sets, improved evidence based disease management and the development of quality improvement working groups. Conclusion: The increasing adoption of medical information technology in health care has helped prevent and track medical errors and adverse events and provide feedback for quality improvement. Much of the information gathered from this technology is stored as electronic health records, which can be easily accessed for research purposes. The utilization of electronic health record data in healthcare research has been proven to improve research efficiency and cost effectiveness, while maintaining data accuracy and quality. Our research suggests that utilizing electronic health records in emergency medicine research is an effective strategy to immediately assess current emergency department practice trends with a goal of improving patient care through staff education.

Keywords: innovations in EM education, electronic health records, quality improvement

\section{P060}

Presentation and outcomes of patients certified in the emergency department under the Ontario Mental Health Act

H. Kang, MD, M. Klingel, MSc, M. Edmonds, MD, MSc, S.L. McLeod, MSc, L. Price, MD, University of Western Ontario, London, $\mathrm{ON}$

Introduction: Acute mental health issues are a common emergency department (ED) problem, and ED physicians must decide which patients require detention for urgent psychiatric assessment. Under the 
Ontario Mental Health Act, a physician may certify a patient on a 'Form 1' if they believe the patient is at serious risk of harming themselves or others. A Form 1 authorizes apprehension and detention of a patient in a psychiatric facility for up to 72 hours in order to allow for a compulsory psychiatric assessment. The objective of this study was to describe the demographics and in-hospital outcomes of patients who had a Form 1 completed by an ED physician and to determine the number of patients who returned to the ED within 30 days of their initial psychiatric assessment and were recertified. Methods: This was a retrospective medical record review of adult patients ( $>17$ years) who had a Form 1 completed in one of two EDs at an academic tertiary care centre (annual census 125,000) over a one-year period (Jan 1, 2012 Dec 31, 2012). A random sample of charts were reviewed for patients who had a second Form 1 completed within 30 days of their initial Form 1. Details on the presentation and disposition of the primary and return ED visits were extracted by trained research personnel. Results: During the study period, 1487 Form 1s were completed by ED physicians for 1148 different patients. Of the 1487 completed Forms, a second Form 1 was completed within 30 days of the initial Form 1 in 140 (9.4\%) patients during the study period. The number of certifications per patient ranged from 1 to 16 . Mean (SD) patient age was 36.8 (15.1) years and $53.9 \%$ were male. $1323(89.0 \%)$ patient encounters had a consult while in the ED, $91.6 \%$ of these were to psychiatry. 927 (62.3\%) patient encounters led to hospital admission. 695 (46.7\%) of the visits had a mood disorder (such as anxiety, depression and adjustment disorders) as the ED discharge diagnosis. Of the 140 charts with a second certificate within 30 days, 47 were randomly selected for further review. Of those, 37 (78.7\%) presented with suicidal ideation, 22 (46.8\%) with attempted self-harm, and $16(34 \%)$ patients had a suicide attempt. Conclusion: This study suggests that a significant number of ED certified patients require recertification within 30 days after their initial psychiatric assessment. This suggests that additional outpatient and urgent psychiatric follow-up may be required for previously formed patients.

Keywords: psychiatry, mental health, emergency medicine

\section{P061}

Establishing a standardized process of gathering evidence-based information to support clinical knowledge development

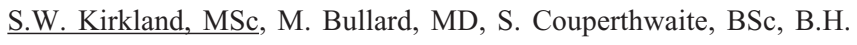
Rowe, MD, MSc, University of Alberta, Edmonton, AB

Introduction: The Emergency Strategic Clinical Network in Alberta, Canada, was created to improve quality emergency care across the province. Developing a standardized process for gathering evidencebased clinical knowledge is important to identify high quality, up-todate information. The objective of this study was to trial five methods of gathering evidence-based answers to support clinical knowledge development. Methods: Physicians were surveyed to identify clinical questions important for the management of alcohol withdrawal and COPD exacerbations in the emergency department. The questions used a Population, Intervention, Control, Outcome, and Design (PICO-D) format. Five sources of information were searched including: guidelines, systematic reviews, Dynamed ${ }^{\mathrm{TM}}$, Up-to-Date ${ }^{\circledR}$, and a rapid review of the literature. The results were then presented to multidisciplinary working group members who, using a seven-point Likert scale, rated their comfort level with the information and their preferred search method. Results of the survey were presented in median and IQR. Results: The comfort levels of the information received by all pre-appraised approaches were rated similarly highly. Systematic reviews were more likely to be selected first to answer the alcohol (5 IQR: 4, 5) and COPD (5 IQR: 4, 7) PICO-Ds over guidelines. For the alcohol PICO-D's, Dynamed was preferred over Up-to-Date (3.5 IQR: 3, 4), while Dynamed and Up-to-Date were equally preferred for the COPD PICO-D's (4 IQR: 3, 5). Conclusion: Systematic reviews were the preferred method for seeking high quality and current clinical information and should become the initial source for identifying evidence-based clinical knowledge to inform clinical decision making. Keywords: clinical decision making, clinical practice guideline, systematic review

\section{P062}

\section{Using clinical grade cadavers for high-fidelity simulation}

G.J. Kovacs, MD, M. Murray, R. Sandeski, B. Vair, MD, J.R. Ross, MD, Dalhousie University, Halifax, NS

Innovation concept: The procedural skills learning adage of 'see one, do one, teach one' is no longer acceptable to learners, educators and the public. Simulation has come a long way as an educational adjunct to improve both procedural skills learning and clinical decision-making. Methods: Access to mannequins and other higher fidelity patient simulators is increasing. These simulators are often costly, can be limited in function, and may not be suitable for learning many procedures. Historically, donated human cadavers have been used for learning purposes, but the fixation process used to preserve these specimens results in rigid unrealistic tissue. Access to non-preserved cadaveric specimens can be challenging for various reasons. Curriculum, tool, or material: Since 2007, the Dalhousie Division of Anatomy in collaboration with the Departments of Emergency Medicine and General Surgery have been using "clinical grade cadavers" (CGC). These CGCs are preserved using a newly adapted embalming technique that retains a natural compliance and texture that is very similar to that of living human tissues. This embalming process preserves the bodies' clinical conditions for up to a month after the donor's death. The result is a truly realistic model that can replace or augment the use of other simulators. At our institution CGCs are now being used in a hospital setting to provide a multidisciplinary simulation environment for learners. Conclusion: Access to CGCs can go a long way to alleviate a "learning opportunity-patient safety conflict" that plagues procedure learning. This conflict arises when high level skill competence is needed to achieve the desired patient outcome but simulator and/or live patient opportunities are either not available or present a patient safety threat. CGCs provide a cost effective, safe option to this challenge.

Keywords: innovations in EM education, clinical cadavers, simulation

\section{P063}

Development and implementation of a novel administration block in a Royal College emergency medicine training program E.S. Kwok, MD, S. Choi, MD, K. Sampsel, MD, J.R. Frank, MD, University of Ottawa, Ottawa, ON

Innovation concept: Traditional Emergency Medicine (EM) training programs underemphasize many nonclinical aspects of the CanMEDS roles and medical practice, reflected by numerous studies reporting many graduating physicians begin their careers with poor understanding of the healthcare system and inadequate preparation for the operational aspects of healthcare delivery. We sought to develop and imple- 
ment a novel structured Administration block in a Royal College EM training program. Methods: We identified essential administrative competencies via needs observed by local experts, in published international studies, and a Canadian needs assessment of EM program directors. Competencies were included if there was consensus that they could not be acquired on existing rotations. Local experts in each of the major topics were recruited as instructors. Multiple instructional methods were deployed as a 2-week experience, including: pre-reading materials, lectures, interactive discussions, and small group applied projects. Validated tools such as the Kraybill Conflict Style Inventory were utilized where available. Evaluations and feedback were elicited for each topic and at the end of the block. Curriculum, tool, or material: The 2-week block was structured into 3 major Parts. Part 1: "Understanding the Canadian Healthcare System" 1.1 Overview of the Canadian Healthcare System 1.2 Health Economics 1.3 International Comparisons 1.4 Politics and Health Advocacy Part 2: "Understanding the Hospital Environment" 2.1 Power, Politics, \& Interdepartmental relationships 2.2 Self-employed Entities working in a Hospital Environment 2.3 Leadership: Theories \& Practice Part 3: "Understanding the Business of Emergency Medicine" 3.1 Departmental Level 3.1.1 ED Crowding, Wait times \& Patient Flow 3.1.2 Process measurement and management 3.1.3 Staffing, scheduling, and running the ED team 3.1.4 Business for Dummies - How to Complete a Healthcare Project 3.1.5 Patient Complaints 3.1.6 Conflict Resolution 3.2 Individual Level 3.2.1 Negotiating an employment contract 3.2.2 Medico-Legal Aspects 3.2.3 Optimizing Patient Flow on Shift 3.2.4 Billing Initial feedback from residents with respect to the proposed curriculum were all positive. Comments consistently reaffirmed the value of explicit training in administrative competencies deemed lacking in previously existing rotations. Conclusion: We developed, implemented, and evaluated a novel Administration \& Business of EM block in a Royal College training program. To our knowledge this is the first Canadian initiative to have an explicit curriculum based on published literature and subject expertise. We believe this template can be easily adopted by any Royal College EM training program and will effectively address core competencies that are traditionally difficult to teach via regular clinical rotations.

Keywords: innovations in EM education, CanMEDS, administration

\section{P064}

Why university athletes choose not to reveal their concussion symptoms during a practice or game

C. Lamfookon, MD, J. Delaney, MDCM, G.A. Bloom, PhD, A. Al Kashmiri, MD, J.A. Correa, PhD, Department of Emergency Medicine, McGill University, Montréal, QC

Introduction: Objective: To determine why athletes decide not to seek medical attention during a game or practice when they believe they have suffered a concussion. Design: Retrospective survey. Setting: University Sport Medicine Clinic. Participants: 469 Male and female athletes from several varsity team sports at two Canadian universities. Methods: Athletes were surveyed about the previous 12 months to identify specific reasons why those athletes who believed they had suffered a concussion during a game or practice decided not to seek attention at that time, how often these reasons occurred and how important these reasons were in the decision process. Results: Ninety two of 469 athletes $(19.6 \%)$ believed they had suffered a concussion within the previous 12 months while playing their respective sport and 72 of these
92 athletes $(78.3 \%)$ did not seek medical attention during the game or practice at least once during that time. Sports in which athletes were more likely to not reveal their concussion symptoms were football and ice hockey. The reason "Did not feel the concussion was serious/severe and felt you could still continue to play with little danger to yourself", was listed most commonly (55/92) as a cause for not seeking medical attention for a presumed concussion. Conclusion: A significant percentage of university athletes who believed they had suffered a concussion chose not to seek medical attention at the time of injury. Improved education of players, parents and coaches about the dangers of continuing to play with concussion symptoms may help improve reporting.

Keywords: concussion, symptoms, reveal

\section{P065}

Epidemiology and outcomes of head injury patients in an urban Ethiopian emergency department

M. Landes, MD, MSc, R. Venugopal, MD, MPH, S. Berman, MPH, A. Azazh, MD, University Health Network, Toronto, ON

Introduction: Head injury is a leading cause of mortality in Africa where many countries are in the early stages of developing pre-hospital, emergency and critical care services. The objective of this study was to characterize the epidemiology and outcomes of head injury at a large urban academic hospital with a newly active emergency medicine program in Ethiopia. Methods: We conducted a prospective cohort of all head injured (including both isolated head injury and multisystem trauma with head injury) patients over 4 months presenting to the Emergency Department of Tikur Anbessa Specialized Hospital, Addis Ababa, Ethiopia. Using a standardized data collection form, demographic and clinical information was extracted from the patient's chart, radiology reports and/or operative reports. Patients were followed until they reached one of the following endpoints: discharge, referral to another hospital, death, or 7 days in the hospital. Consent for study participation was obtained from the patient or substitute decision maker. Results: Overall, 204 patients with head injury were enrolled of which $53(26.0 \%)$ had multisystem trauma. The majority of head injuries occurred in those 16 to 30 years old $(51.0 \%)$ and among men (86.8\%). Over half of all head injuries were a result of road traffic accidents (RTAs): $24.5 \%$ in pedestrians struck by a vehicle and $25.7 \%$ as a driver or passenger. Other mechanisms included: assault with blunt injury $(38.2 \%)$ or penetrating injury $(2.5 \%)$ and falls $(17.7 \%)$. Head injury severity (as per the Glasgow Coma Scale (GCS)) on presentation was as follows: 108 patients had mild injury (GCS 13-15; 53.0\%), 39 with moderate (GCS 9-12; 19.1\%), and 51 with severe (GCS 3-8; 25.0\%). Overall, $149(73.0 \%)$ patients were discharged from hospital, 34 $(16.7 \%)$ were referred to another hospital, and 21 patients died (10.3\%). The majority (62.6\%) of deaths occurred within 48 hours of admission to the emergency department. In multivariable analysis, death was significantly associated with the following characteristics on presentation to the emergency department: age over 60 years (aOR 68.8, 95\%CI 2.02329.0, $\mathrm{p}=0.02$ ), GCS less than 9 (aOR 14.8, 95\%CI2.2-99.5, $\mathrm{p}=0.01$ ), fixed bilateral pupils (aOR 39.1, 95\%CI4.2-362.8, $\mathrm{p}<0.01$ ) and hypoxia (oxygen saturation $<90 \%$; aOR $14.2 \%, 95 \% \mathrm{CI} 2.6-123.9$, $\mathrm{p}=0.01$ ). Conclusion: Head injury after trauma represents a significant risk of morbidity and mortality in this setting in Ethiopia, for which the ongoing development of emergency and surgical services may improve outcomes. Appropriate identification and services for patients at risk of mortality may guide this continued development. 
Keywords: head injury, trauma, outcomes

P066

Emergency department outpatient management of deep vein thrombosis and pulmonary embolism: management and outcomes S. Law, BSc, D. Ghag, Pharm D, R. Stenstrom, MD, PhD, E. Grafstein, MD, D.R. Harris, MD, MHSc, Department of Pharmacy, St. Paul's Hospital, Vancouver, BC

Introduction: Many patients with venous thromboembolism (VTE) can be managed in the emergency department as outpatients without admission to hospital. However, current standard treatment is low-molecular weight heparins as bridging therapy until a therapeutic INR is obtained on oral anticoagulants. Our objective was to determine the impact on ED resources (days of therapy, recurrence, readmissions) for managing VTE patients in the outpatient setting. Methods: This was a retrospective medical records review study. Patients were identified from the St. Paul's Hospital and Mt. St. Joseph's Hospital Emergency Department Discharge Database. St. Paul's is a large academic inner city hospital with an ED census of 78,000 visits; Mt. St. Joseph's is an acute care community hospital with a census of 25,000. Patients were identified from a larger VTE cohort. Included patients were those diagnosed with VTE (either PE or DVT) treated exclusively in the ED setting. Excluded patients were those admitted to hospital, untreated, lost to follow up (including single visits), with incomplete data, those with renal dysfunction, with active cancer, or pregnant. Charts were reviewed from the period Jan. 1, 2010 to Dec. 31, 2012. Data was collected on patient demographics, location of VTE, management within the ED, and outcomes. Outcomes were determined from linkage to a Regional Database (recurrence, readmissions, major bleeding) and Vital Statistics, with a follow-up period of 7 months. Providence Health Care REB granted ethical approval. Results: 400 radiologically confirmed cases of VTE were identified during the study period; of those, 201 were included in the study. Average age was 55; 63\% were male. Of the cohort, 183 (91\%) were diagnosed with DVT; 18 with PE (9.0\%). On discharge, only $72.1 \%$ (145/201) were appropriately anticoagulated (defined as two consecutive INR levels between 2 and 3). VTE patients returned daily to the emergency department an average of 7 times (IQR 6 - 9) for anticoagulation (range: 3 to 21 visits). Of the cohort, 2 / 201 $(1.0 \%)$ died within 7 months after diagnosis. $18 / 201(9.0 \%)$ were readmitted to hospital during 7 month follow up; $4 / 18(22 \%)$ related to VTE or complications of anticoagulation. 7 / 201 (3.5\%) had a drop of $20 \mathrm{mg} / \mathrm{dL}$ in hemoglobin while on therapy. Conclusion: In this cohort, the management of VTE patients through the emergency department could be improved. Opportunities exist for improving rates of appropriate anticoagulation. The use of new oral anticoagulants within the ED for outpatient VTE therapy may improve rates of appropriate anticoagulation and reduce the burden on ED resources.

Keywords: venous thromboembolism, treatment

\section{P067}

Examining the current health care milieu of the new Ethiopian emergency medicine system: a qualitative analysis of Ethiopian emergency medicine residents

J. Lockwood, MD, A. Azaj, MD, S. Berman, MPH, S. Vaillancourt, MD, MPH, J. Maskalyk, MD, M. Landes, MD, MSc, Division of Emergency Medicine, Department of Medicine, University of Toronto, Toronto, ON
Introduction: Despite a significant burden of acute illness, infrastructure for emergency medical care remains in the early stages of development in Ethiopia. The Toronto-Addis Ababa Academic Collaboration in Emergency Medicine (TAAAC-EM) is a collaboration between Addis Ababa University and the University of Toronto to address the design and implementation of the first Ethiopian Emergency Medicine (EM) resident training program. The Collaboration seeks to build national emergency care capacity, but similar programs elsewhere have had unintended consequences on human resources migrations. The objective of this study was to improve understanding of the health care and human resources issues that new graduates will be encountering as Ethiopia's first emergency medicine physicians. Furthermore, we wished to explore emergency medicine residents' perspectives on factors affecting retention post graduation. Methods: A series of semistructured qualitative interviews were conducted with six Ethiopian EM residents in October 2012 in Addis Ababa. Two residents were interviewed from each academic year of the three-year program. The interviews, after verbatim transcription, were imported into Weft QDA software for coding and analysis. Themes and sub-themes were identified and thematic analysis of the data was conducted with the retrieved coded data. Finally, a narrative account of the findings was developed. Results: A number of push and pull factors were identified pertaining to graduate retention including: financial, workplace (including concerns about supplies and resources), career expectations and current unmet training needs. All residents expressed a desire to remain in Ethiopia, and the interviewees identified potential solutions to mitigate forces encouraging emigration, including financial incentives, improvements to workplace conditions and continuing education. Cohesive forces were identified including social familiarity and return to service agreements that encourage graduates to pursue careers within Ethiopia. Conclusion: A number of 'push' and 'pull' forces were identified which may affect retention of the first cohorts of emergency medicine graduates in Ethiopia. Solutions regarding retention of emergency medical professionals must be specific for the Ethiopian context. The attitudes and opinion of other important stakeholders, including politicians and health care administrators, must be ascertained for a more complete understanding of the human resources context for this developing emergency medicine system.

Keywords: administration, postgraduate education, international

\section{P068}

Just in Time ATLS: training for trauma during disasters

T. Loubani, MD, A. Obaid, MD, N. Parry, MD, M. Al-Attar, MD, S.L. McLeod, MSc, M. Klingel, MSc, Al Shifa Hospital, Gaza, Palestinian territories

Innovation concept: There is a paucity of research on the rapid teaching and deployment of trauma care in disaster scenarios for otherwise experienced physicians. However, such educational programs are critical to rapidly scaling up healthcare provider resources in times of crisis. The proposed work expands on such a program that was developed to optimize the care of mass casualties sustained during air strikes and shelling in Gaza in November 2012. Early in the disaster, it was observed that many physicians had adequate technical skills, but lacked an organized, effective approach to trauma patients. The objective of this study is to further evaluate this previously piloted program among physicians using a training approach we term "Just-in-time 
ATLS" (JIT-ATLS). Methods: Study design. This is a single-blinded randomized trial. Participants. Participants will be recruited among physicians registered for a regular ATLS course at Al-Shifa Hospital, Gaza and London Health Sciences Centre, Canada. Participants will be randomized to either the intervention or control arms. Intervention. Study participants will be given a JIT-ATLS training course. The first iteration of JIT ATLS was developed in collaboration with senior trauma physicians in Al Shifa Hospital, Gaza and London Health Sciences Centre, Canada. The JIT curriculum is a 4-hour mixed theoretical and practical session that focuses on team dynamics, communication, primary and secondary surveys, and immediate interventions. Specific technical skills (e.g., chest tubes) are assumed. Control. Control participants will participate in a normal ATLS course. Evaluation. Testing will be done pre- and post-training. Evaluation consists of several realistic mock trauma codes, which will be video-recorded for assessment by trained evaluators blinded to the study arm of the participants. Outcomes will include a list of critical and non-critical items, as well as time to completion. Curriculum, tool, or material: Study not yet complete. Conclusion: Study not yet complete.

Keywords: innovations in EM education, trauma, international medicine

P069

Is exposure to major trauma during medical training adequate for emergency physicians in New Brunswick?

D. Lukanovic, J. French, BSc BM Dip IMC RCS Ed, S. Benjamin, BN, J. Fraser, BN, P.R. Atkinson, MD, Dalhousie Medicine, St. John, NB

Introduction: The management of a major trauma is challenging and is the subject of ongoing scrutiny at provincial and national levels. Learning curves theory suggests that proficiency improves as competency use increases. Without frequent use, competency can be lost as "skill fade" occurs. This may be reflected in data suggesting that increasing departmental exposure to major trauma improves major trauma outcomes. Using data from New Brunswick (NB) Emergency Rooms and Trauma New Brunswick (TNB), the objective of this project was to determine whether medical clerks and emergency medicine residents are receiving enough trauma exposure to become proficient in the management of major trauma patients. Methods: A data extract from TNB was used to determine the count of major trauma cases (defined as patients with an Injury Severity Score of 15) presenting to emergency departments at the Saint John Regional Hospital (SJRH) and The Moncton Hospital (TMH) in 2011-2012. The total number of emergency room visits at all emergency departments in NB was also obtained. The proportion of Major Trauma attendances for SJRH and TMH was calculated. This proportion was then used to estimate the count of cases of major trauma presenting to all other emergency departments in NB that do not collect injury scored data. The average exposure to major trauma cases for residents and medical students was then estimated using typical schedules for both groups. Results: Emergency medicine trainees will see approximately 11 cases of major trauma over their entire medical school and residency training in NB. A physician working a typical schedule of four eight-hour shifts per week will see 12 cases per year of major trauma. Given the methodological limitations of this study, these are likely to be overestimates. Conclusion: The current literature in medical education suggests that only seeing 11 cases of major trauma over the course of medical training may not be sufficient for an emergency physician to become proficient in major trauma management. This exposure also suggests that maintaining competency may be challenging. Existing training could be augmented with simulation to help learners become skilled at trauma management. Departmental systems should be designed assuming that users may provide care to these critically ill patients relatively infrequently, which would support the use of checklist, refresher tasks and maximising learning through thorough case review.

Keywords: trauma, medical education, emergency medicine

P070

Development of a national simulation curriculum: what do residents think?

A.H. McConnell, BSc, MKin, MD, C. Filipowska, MD, PhD, S.L. McLeod, MSc, J.R. Frank, MD, K. Woolfrey, MD, University of Western Ontario, London, ON

Introduction: As residents progress through their training, they acquire skills in triage, assessment, intervention, resuscitation and management of a broad range of presentations in the emergency department. Assessment of resident ability is becoming competency based; however, there is sparse literature to inform this educational model with regard to simulation. While many emergency medicine (EM) programs have integrated simulation training into their competency-based models, there is a void regarding a structured simulation curriculum for EM trainees. The objective of this study was to understand resident perspective on the content of an EM post-graduate simulation curriculum. Methods: EM residents at a Canadian university were invited to participate in an online survey to assess each objective outlined in the Royal College of Physicians and Surgeons of Canada Objectives of Training (OOT) for individual suitability of each objective for inclusion in the curriculum. Participants assessed each OOT and determined whether it would be best taught by simulation. Consensus was achieved with $70 \%$ agreement. Results: $87 \%$ of eligible residents responded, with an equal distribution of senior and junior participants. Consensus was achieved in $221(50 \%)$ of the 442 OOTs. Of those, $89(40.3 \%)$ OOTs achieved agreement to be included and 132 (59.7\%) OOTs achieved agreement to be excluded from the curriculum. The content areas agreed upon for inclusion were almost exclusive to the competency of Medical Expert (87/89; 98\%), which represented 73\% (87/119) of the Medical Expert objectives. These focused on medical and surgical resuscitation, with emphasis on procedural skills, toxicology, and environmental emergencies. In contrast, none of the OOTs from Communicator, Health Advocate, Professional, Scholar and Manager categories were included. Conclusion: Residents at an academic teaching centre identified several areas that would be best taught by simulation to compliment their didactic and experiential learning. Program directors should consider topics and skills that may be optimally delivered via simulation training when designing future curriculum.

Keywords: simulation, medical education, residents

\section{P071}

Lumbar puncture practices by emergency physicians: a multicenter study

N. Brown, MD, M. Ng, MD, E. Lang, MD, S. Pytka, MD, A. Pat, BSc, Y.S. Liu, BSc, A. McRae, MD, University of Calgary, Calgary, AB

Introduction: Post-dural-puncture headache (PDPH) is a known complication of lumbar puncture (LP). Needle gauge and design are modifiable risk factors for PDPH. Many standard LP kits include a 22G 
Quincke needle. Incidence of PDPH with this size and style of needle is as high as $36 \%$. Use of atraumatic needles, such as the Whitacre, reduces this incidence to $0.6-4 \%$. The objective of this study was to survey the knowledge and practice pattern of ED physicians as to their choice of needle for LPs. Methods: We sent a ten-question survey to the four adult EDs in the Calgary region (population 1.2 million, combined annual ED census 230,000). Questions addressed the type of needle used; risk of PDPH with different needle type and gauges; reason for needle choice; PDPH treatment options; and knowledge of availability of different needle types or introducers to aid with insertion of non-cutting needles. Data were analysed using descriptive statistics. Results: 101 of 178 physicians $(56.7 \%)$ responded. $75 \%$ of respondents were attending physicians, and $25 \%$ were residents. $46 \%$ used the $22 \mathrm{G}$ Quincke, 36\% used a 25G Whitacre (non-cutting), 10\% used either and $8 \%$ used another needle. Reasons cited for choice of the Quincke included convenience (the needle included with the kit), easier skin puncture, and faster CSF flow. Respondents estimated PDPH risk to be $12 \%$ with the $22 \mathrm{G}$ Quincke and $5 \%$ with the $25 \mathrm{G}$ Whitacre. Respondents estimated that $7.2 \%$ of patients receiving an LP in the ED may go on to receive a blood patch. $80 \%$ of respondents wanted a $22 \mathrm{G}$ Whitacre needle included in the LP kit. Over-the-counter analgesics and caffeine were the first line of treatment for PDPH for 35\% of respondents whereas blood patch was the first line for $17 \%$. Conclusion: Emergency physicians most commonly used the $22 \mathrm{G}$ Quincke needle included in the LP kit. Respondents underestimated the incidence of PDPH with 22G Quincke needles, which may represent an important knowledge gap. Including a non-cutting spinal needle in LP kits may be a simple intervention that would reduce a preventable complication of LPs.

Keywords: lumbar puncture, post-dural puncture headache, headache

P072

Mortality and outcomes for frequent users of emergency departments

J. Moe, BASc, MA, MD, S.W. Kirkland, MSc, L. Assoignon, BScN, S. Campbell, MLS, B.H. Rowe, MD, MSc, University of Alberta, Department of Emergency Medicine, Edmonton, AB

Introduction: Frequent Emergency Department (ED) users are an important patient group; they represent a disproportionate resource burden and a potentially vulnerable group. Multiple studies have attempted to characterize frequent users and examine interventions designed to identify and address their healthcare needs. This evidence has been aggregated in three systematic reviews to date, none of which specifically addressed health outcomes. This review was designed to examine definitions of frequent ED users and important health outcomes (e.g., mortality, admissions, health service use, etc.). Methods: A comprehensive search of eight electronic databases and grey literature search combining terms for "frequent use" and "emergency department" was developed with a medical librarian. Using a two-step process for potential relevance (title, index terms) and inclusion (full article), three independent reviewers assessed studies. Disagreements were resolved by a fourth author. Data extraction and quality assessment will be performed by two independent reviewers. Subgroup analyses are planned based on: various definitions of frequent use, receipt of standard of care vs. clinical/other interventions, similar parameters for mortality, comparable outcome measures, and duration of follow-up. Results: The initial search strategy yielded 5980 articles; 1970 duplicates were removed, leaving 4010 citations. After the initial pre-screen, 2161 citations remained and were screened by title and abstract, yielding 378 articles. 97 were editorials and were excluded, while 14 articles could not be located. Overall, 267 articles were retrieved for full review. Further results are pending data extraction. Conclusion: While a large number of studies exist relating to frequent ED users, the number of research studies examining mortality appears to be small; other health outcomes are reported more commonly. In addition, definitions of what makes someone a frequent user varied considerably. Standard definitions of frequent users and focus on health outcomes could provide clarity to this field. Once completed, this systematic review will fill an important gap in the current literature on this topic.

Keywords: frequent emergency department users, mortality, outcome assessment

\section{P073}

Distal radial fractures: adequacy of reductions performed in the emergency department

R. Montazeri, MD, P.R. Atkinson, MD, J. Fraser, BN, S. Benjamin, BN, J. Wagg, MD, J. Mayich, MD, J. Swan, MD, H. King, MD, Department of Family Medicine Residency Program, Dalhousie University, Halifax, NS

Introduction: Distal radial fractures remain the most commonly encountered fracture in the Emergency Department (ED). Their initial management by Emergency Physicians (EP) poses considerable resource allocation. Sedation and manipulation in the acute setting frequently requires multiple hospital team members, including radiological reassessment and orthopedic follow-up. A literature search in methods of approach revealed little in consensus or effectiveness of current practices. Methods: We performed a retrospective study to evaluate the adequacy and re-displacement rates of reductions performed by EPs. The need for further interventions at follow-up was also examined. Secondary outcomes of interest were demographic relationships of age, sex, anesthetic used, casting type, time of initial presentation in $\mathrm{ED}$, need for multiple reduction attempts, and choice of imagining (Xray, C-arm or U/S) as predictors of a satisfactory outcome. Results: 73 patients who received procedural sedation and manipulation for a distal radial fracture were included in the study. Radiological images performed at initial assessment, post reduction, and on clinic follow up of included patients were reviewed by a panel of orthopedic physicians and assessed for evidence of displacement. Rates of intervention at follow up were low and were not associated with any single predictive factor. Conclusion: The majority of distal radius reductions performed by emergency physicians are satisfactory and re-manipulation rates at follow up are low.

Keywords: trauma, fractures, distal radius

\section{P074}

Evaluation of advanced life support experiences by clerkship students

M. Peirce, MD, M. Moss, MD, F. Myslik, MD, M. Rossoni, MD, M. McWilliam, MD, C. Cookson, MD, M. Lewell, MD, University of Western Ontario, London, ON

Introduction: In Canada, a recent expert panel outlined the core competencies that should be included in the emergency medicine clerkship experience. One of these recommendations included an understanding of the investigations, treatment, and differential diagnosis of cardiores- 
piratory arrest and cardiac arrhythmias, which are covered in advanced cardiovascular life support (ACLS) training. However, there has been little research examining the frequency of these ACLS events encountered by medical students during their clinical clerkship. The objective of this study was to quantify the number of times third year medical students were exposed to advanced cardiovascular life support events during their 12-month core clinical clerkship experience. Methods: This was a prospective, observational study of third year medical students affiliated with an academic tertiary care centre to document the number of episodes of stable tachycardia, unstable tachycardia, unstable bradycardia and cardiac arrest events they encountered over their 12-month core clinical clerkship. Students were asked to use the definition of each arrhythmia contained in the "Cardiovascular Life Support Provider Manual" by the American Heart Association to standardize the data collection. Results: 100 medical students participated in this study. On average, students witnessed a mean (SD) of 9.0 (10.3) stable tachycardia events, 2.8 (3.3) events of unstable tachycardia, 1.6 (1.8) events of bradycardia, and 1.2 (1.6) events of cardiac arrest during the study period. Conclusion: Medical students are frequently exposed to arrhythmias that are treated according to ACLS guidelines in their clerkship year. Medical schools may consider introducing ACLS training earlier in medical education so that medical students can better understand ACLS principles when witnessing or participating in resuscitations during their clerkship.

Keywords: ACLS, clerkship, education

\section{P075}

The use of a new feedback form in the pediatric emergency department: incorporating CanMEDs and self-reflection

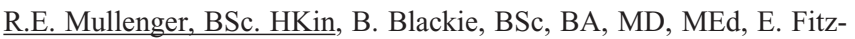
patrick, Faculty of Medicine, Dalhousie University, Halifax, NS

Introduction: Feedback is an essential component in the development of clinical and professional skills in medicine. Self Reflection is another essential part of professional development and both these elements are now emphasized in medicine at all levels of training. The CanMEDs competencies are an integral, core part of Canadian postgraduate medicine programs and therefore an innovative feedback form that combines CanMEDs competencies with feedback and selfreflection has been developed for use in a tertiary care pediatric emergency department. The purpose of this qualitative study is to explore experiences with the form to determine if including the CanMEDs competencies and self-reflection has provided a richer learning with feedback experience for residents and physicians in the pediatric emergency department. Methods: Semi-structured focus groups and interviews were conducted with pediatric emergency medicine physicians and residents rotating through the department from different programs in order to look at attitudes towards feedback and the CanMEDs roles in this context. Grounded theory guided the analysis of the focus group and interview data. Thematic analysis identified overarching themes including value of feedback, lack of understanding of the CanMEDs roles, feedback needs and self-reflection. Results: Overall the feedback form has been well received, acting as a framework to guide selfreflection and enhance end of rotation ITERS. Overarching themes included value of feedback, lack of understanding of the CanMEDs competencies, feedback needs and self-reflection. Pediatric residents differed from Emergency Medicine residents in attitudes towards the form and the richness of self-reflection tracked on the form. Residents' feelings towards CanMEDs stem from a sense of artificial separation of the competencies and quality of feedback being staff dependent. Conclusion: This study enhances the current literature and identified that staff and residents require further training around the CanMEDs roles. An approach to future use of the form has been developed. Adjustments are recommended to best prepare residents to excel in practice and provide excellent patient care and include minor modifications to the structure of the form, suggestions for developing faculty training and the adoption of the feedback form by other undergraduate and postgraduate programs.

Keywords: medical education, CanMEDS, feedback

\section{P076}

Screening for post-traumatic stress disorder after injury in the pediatric emergency department - a systematic review

J. Odenbach, MSc, A. Newton, PhD, R. Gokiert, PhD, C. Falconer, BScN, C.E. Courchesne, BSc, S.J. Curtis, MD, MSc, Women's and Children's Health Research Institute, Edmonton, AB

Introduction: Pediatric injury is highly prevalent and has significant impact both physically and emotionally. The vast majority of pediatric injuries are treated in emergency departments, where treatment of physical injuries is the main focus. In addition to physical trauma, children often experience significant psychological trauma, which often goes unrecognized. The development of posttraumatic stress disorder is common, sometimes even after seemingly mild trauma. The consequences of failing to recognize childhood posttraumatic stress disorder are stark and extend into adulthood. Currently, screening guidelines to identify children at risk for developing for these stress disorders do not exist in the pediatric emergency setting. Methods: The goal of this systematic review is to summarize evidence on psychometric properties, diagnostic accuracy, and clinical utility of screening tools that identify or predict posttraumatic stress disorder in children and adolescents with acute injury. Results: 10,415 articles were screened by title/abstract by two independent reviewers and 228 potentially relevant articles were retrieved in full text. Interrater reliability (kappa) score of 0.833 was calculated for article screening. Screening tool characteristics; including type of instrument, number of items and administration time as well as quantitative diagnostic data (specificity, sensitivity, positive and negative likelihood/odds ratios, as appropriate) were compiled for 89 screening tools. Of these tools, we identified 1 immediate use risk assessment tool and 88 symptom assessment tools including 3 clinician-administered screening tools, 56 self-report screening tools, 7 parent-report screening tools and 22 (semi-) structured diagnostic interviews. Conclusion: Numerous posttraumatic stress disorder screening tools exist and span a wide variety of clinical pediatric settings. Although most tools are overly complex and lengthy to be suitable for use in the emergency department, a small number of simple/abbreviated tools exist and further research is warranted to determine their efficacy in the acute pediatric injury setting.

Keywords: systematic review, screening tools, post-traumatic stress disorder

\section{P077}

Factors associated with having a family physician among visitors of emergency departments in a Canadian urban setting

M.B. Ospina, PhD, R. Chetram, S.W. Kirkland, MSc, B. Voaklander, A. Davidson, T. Nikel, B. Holroyd, C. Villa-Roel, S. Couperthwaite, BSc, G. Cummings, MD, D. Voaklander, PhD, B.H. Rowe, MD, MSc, University of Alberta, Edmonton, AB 
Introduction: The emergency department is sometimes used as an alternative to primary care when patients cannot see their family physician (FP) or do not have one. Limited access to primary care services may be a contributing factor in ED overcrowding. This study evaluated the frequency and factors associated with having a FP among ED users in an urban setting. Methods: A standardized questionnaire was administered to 1,408 randomly selected patients aged $>17$ years, with non life-threatening conditions presenting at three EDs in Edmonton (Canada). Backward logistic regression explored associations among sociodemographic factors and having a FP. Results: Of 1,408 patients who completed the survey, $74.4 \%$ had a FP. Among those with a FP, 95.1\% had a FP visit over the last 6 months. Reasons for not having a FP were related to difficulties with finding a FP $(40.5 \%)$, no interest in finding one $(31.6 \%)$, and recent relocation in the city $(21.8 \%)$. Factors associated with not having a FP were age $(<25$ years odds ratio $[\mathrm{OR}]=0.1 ; 95 \%$ confidence interval $[\mathrm{CI}]$ : $0.07,0.2$ ), marital status (single; $\mathrm{OR}=0.5 ; 95 \% \mathrm{CI}$ : $0.3,0.7$ ), race (non-Caucasian; $\mathrm{OR}=0.6 ; 95 \% \mathrm{CI}: 0.4,0.8$ ) and being a smoker $(\mathrm{OR}=0.7 ; 95 \% \mathrm{CI}: 0.5,0.9)$. Conclusion: Not having a FP is associated with important socioeconomic factors that are likely to trigger further inequities at the primary care level and contribute to the use of the ED as first and repeat point of contact within the health care system.

Keywords: emergency department overcrowding, access to care, emergency services

\section{P078}

Awareness and use of health link prior to an emergency department visit in a Canadian urban setting

T. Nikel, M.B. Ospina, PhD, S.W. Kirkland, MSc, R. Chetram, G. Cummings, MD, B. Voaklander, A. Davidson, B. Holroyd, E. Cross, C. Villa-Roel, S. Couperthwaite, BSc, D. Voaklander, PhD, B.H. Rowe, MD, MSc, University of Alberta, Edmonton, AB

Introduction: Health Link Alberta is a $24 / 7$ toll-free number that provides Albertans health advice on the most appropriate care, including ED visits. This study assessed predictors of awareness and use of Health Link prior to ED visits in Edmonton, Alberta. Methods: A standardized questionnaire was administered to randomly selected patients aged $>17$ years, with non life-threatening conditions presenting at three urban EDs. Backward logistic regression explored associations among sociodemographic factors and awareness and use of Health Link prior to an ED visit. Results: Of 1,408 respondents, $64.3 \%$ were aware of Health Link, but only $8.1 \%$ reported they called prior to their ED visit. Health advice influenced the decision to visit the ED in $78 \%$ of callers. Females (odds ratio $[\mathrm{OR}]=2.7 ; 95 \%$ confidence interval $[\mathrm{CI}]: 2.1,3.4)$, middle-aged individuals $(26-40$ years $\mathrm{OR}=2.3 ; 95 \% \mathrm{CI}: 1.6,3.3 ; 41$ - 55 years $\mathrm{OR}=1.7 ; 95 \% \mathrm{CI}: 1.2,2.6)$, and those with a family physician $(\mathrm{OR}=$ 2.2; 95\% CI: 1.6, 3.0) were more likely to be aware of Health Link. Health Link awareness was lower among non-Caucasians $(\mathrm{OR}=0.5$; 95\% CI: 0.3, 0.6). Predictors of Health Link use were being female $(\mathrm{OR}=2.6 ; 95 \% \mathrm{CI}: 1.6,4.2)$ and not fully employed $(\mathrm{OR}=1.6 ; 95 \%$ CI: 1.02, 2.5). Conclusion: Awareness of Health Link is high; however, use prior to an ED visit is low. More research is needed to understand how sociodemographic factors influence health literacy and decisions to access ED services.

Keywords: knowledge translation, access to care, emergency department

\section{P079}

Outcomes of emergency department visits of aboriginal peoples diagnosed with chronic obstructive pulmonary disease in Alberta M.B. Ospina, PhD, D. Voaklander, PhD, A. Senthilselvan, PhD, M.K. Stickland, PhD, B.H. Rowe, MD, MSc, M. King, PhD, University of Alberta, Edmonton, AB

Introduction: Emergency department (ED) encounters for exacerbations of chronic illnesses may contribute to health inequalities among Aboriginal peoples. This study compared ED length of stay (LOS) and adverse outcomes in Aboriginal peoples with COPD relative to their Aboriginal counterparts in Alberta. Methods: Retrospective administrative health services data from Alberta were used to identify ED visits by Aboriginal peoples and non-Aboriginals diagnosed with COPD between 2002 and 2009. Median LOS (interquartile range [IQR]) and proportions of visits that resulted in premature departure from the ED (leaving against medical advice [LAMA] or without being seen [LWBS]) were described. Results: Overall, 35,951 ED visits (76.8\% in Aboriginal peoples and $23.2 \%$ in non-Aboriginals) occurred during the follow-up. The median ED LOS of Aboriginal peoples was significantly shorter than in the non-Aboriginal group (1 hour; IQR: 0,4 vs 2 hours; IQR: 1,$5 ; \mathrm{p}<0.0001)$. More Aboriginal patients were discharged compared to non-Aboriginals $(77.1 \%$ vs. $71.3 \%)$, whereas more ED visits by non-Aboriginal patients culminated in hospital admission ( $26 \%$ vs. $17.2 \%$ ). Compared to the non-Aboriginal group, more visits in the Aboriginal group culminated in LAMA (3.9\% vs. $2.2 \%$ ) or LWBS $(0.8 \%$ vs. $0.2 \%)$. Conclusion: Shorter ED LOSs in Aboriginal peoples with COPD and more ED discharges suggests a milder disease; however, the high proportion of encounters resulting in premature departure from the ED suggests considerable unmet needs. Further studies are required to explore the outcomes of ED visits and other characteristics of Aboriginal who are familiar faces in Canadian EDs. Keywords: length of stay, aboriginal peoples, LWBS

\section{P080}

Leadership in crisis situations: merging the interdisciplinary silos H. Paquin, MDCM, I. Bank, MDCM, L. Nguyen, MDCM, MSc, R. Fisher, MDCM, M. Young, PhD, P. Nugus, PhD, McGill University Department of Pediatrics, Montréal, QC

Introduction: Crisis situations in medicine, while relatively infrequent, pose a significant threat to patient safety. When these rare crisis situations occur, patient outcome is critically dependent on a coordinated interprofessional and interdisciplinary health care team for effective and timely intervention. Traditionally, a single medical specialist assumes the role of team leader. However, certain clinical situations require the simultaneous involvement of multiple specialists, who are individually trained to be team leaders. As a result, the potential rise in communication errors and delays in effective and safe patient care can lead to medical errors and poor patient outcomes. The aim of this study is to determine how different specialties involved in crisis resource management approach the role of leader, in order to better define leadership, and improve interdisciplinary (from various specialties) leadership communication and education. Methods: Our qualitative study features purposively sampled, semi-structured interviews with 27 clinicians, from three different specialties involved in crisis resource management in three different centers across the country; Otolaryngology, Anesthesia and Pediatric Emergency Medicine. Interview questions were based on a literature review and peer-reviewed by an expert 
group. Each interview was recorded and is currently being transcribed for thematic analysis. Results: Transcripts are still being analyzed for emerging themes. We predict patterns of similarity and differences across various dimensions of the sampled clinicians. We expect to identify physicians' beliefs in what constitutes decisive leadership. We anticipate finding that appropriate leadership transitions during crisis intervention is perceived to be more a function of the scenario than the personality of individual clinicians and their relationships in a particular hospital. We envisage that interdisciplinary communication and identification of roles will be aided by practicing using simulation as a modality. Conclusion: The preliminary findings suggest that physicians have differing approaches to leadership in crisis situations and that leadership may need to be dynamic, fluid and transferred among physicians on a case by case basis. This study highlights the need for a potentially shift in the way crisis team training is taught. A curriculum including interdisciplinary leadership skills and transference of leadership in crisis situations should be considered.

Keywords: leadership, crisis resource management, interdisciplinary team training

\section{P081}

Effects of paramedic education and novel oral analgesia directive on prehospital pain assessment, documentation and management H. Park, MD, A. Nayar, MD, M. Longeway, M. Welsford, BSc, MD, McMaster University, Hamilton, ON

Introduction: An effective prehospital analgesia practice includes pain evaluation, documentation and management. In Ontario, Advanced Care Paramedics can administer opioids for pain, while Primary Care Paramedics (PCPs, similar to EMT-Ds) cannot. Recently, a novel oral analgesia protocol was implemented among PCPs in Haldimand County, a rural area of Ontario with a population of 45,000 . This protocol allowed administration of acetaminophen and ibuprofen in patients with isolated extremity injuries. This study evaluated paramedic assessment and management of pain before and after an online education module on prehospital pain and implementation of the oral analgesia protocol. Methods: Retrospective review of ambulance call reports with trauma codes for extremity injuries between January 1, 2011 to June 30, 2011 (before) and between January 1, 2013 to September 30, 2013 (after) was performed. The online education module was completed in the summer of 2012 and the oral analgesia directive was implemented in September 2012. Assessment of pain as reflected by pain scale documentation was abstracted. In the after group, the rate of administration of analgesia in eligible patients as well as post analgesia pain scale documentation was also abstracted. Prespecified subgroup analysis was performed to ascertain possible differences in analgesia administration based on age, gender, initial vital signs and transport time of patient. Results: Sixty-seven pre-education and 134 post education ACRs were retrieved that met the inclusion criteria. There was a significant improvement in pain scale documentation before and after the educational intervention (19\% vs $47 \%$, $\mathrm{p}<0.001)$. Documentation rate was significantly correlated with oral analgesia administration $(\mathrm{p}=0.001)$. There were no statistically significant relationships between a patient's age, gender, initial vital signs or transport time and pain scale documentation or administration of oral analgesia. Oral analgesia was correctly administered to $28 \%$ (27/97) of eligible patients. Of those that received analgesia, pain scale re-assessment was documented in $26 \%(8 / 31)$ of patients. Conclusion: Selfdirected online education module on prehospital pain and a novel anal- gesia protocol appear to be an effective method of improving pain assessment documentation among PCPs. No demographic bias was observed for assessment and management of pain. Ongoing paramedic knowledge translation is required to improve use of the directive.

Keywords: emergency medical services, analgesia, education

\section{P082}

Injuries related to falls in the elderly in British Columbia: Kelowna General Hospital's experience

B.A. Parker, BSc, A.P. Leung, E. Ertel, J. Eppler, MD, M. Ertel, MD, The Kelowna General Hospital, Kelowna, BC

Introduction: Falls are common in the elderly with approximately one in three individuals over age 65 experiencing at least one fall annually. Such falls often result in injury and may lead to hospitalization or even death. Falls also place a significant burden on Canada's health care system accounting for more than one third of all injury related hospitalizations. The Kelowna General Hospital (KGH) implemented an injury surveillance program in order to identify injury patterns and common causes of falls in the elderly. Methods: In 2012, KGH joined the 16 other hospitals in the Public Health Agency of Canada's Canadian Hospitals Injury Reporting \& Prevention Program (CHIRPP). At KGH, between March 2012-June 2013, trained volunteer undergraduate research assistants identified, obtained consent from, and interviewed patients over the age of 65 seen in the ED's Minor Treatment (MT) area for a fall-related injury between the hours of 10:00-22:00, seven days a week. A standard CHIRPP questionnaire was used to obtain patient demographics as well as information relating to the cause and location of the injury. Other patient information including CTAS level, medical history, medications, was also collected. The prevalence of various risk factors and injury types were calculated. Results: During the study period it was found that over $60 \%$ of all study patients were triaged as a CTAS 2 or 3. This is significant as the MT area at KGH is staffed primarily by LPNs, with a limited scope of practice compared to an RN. Fewer than $2 \%$ of study patients were CTAS Level 5 . The admission rate for these study patients in the MT area was $27 \%$ (the overall admission rate from the ED is approximately $22 \%$ ). Also, in the Okanagan the population of males to females is close to 50:50. However, in our study population $73 \%$ of patients were female. Nearly $50 \%$ of all study patients presented with a fracture, and $14 \%$ had 2 or more injuries upon presentation. Fractures were more common in females vs. males $(56 \%$ vs. $28 \% \mathrm{p}<.001)$. Wrist fractures were most common, occurring in $15 \%$ of women and only $2 \%$ of men $(\mathrm{p}=.002)$. A documented prior history of a fall was found in $24 \%$ of all study patients. Of patients who were admitted, 33\% had a prior fall history. Conclusion: Patients over 65 with falls are often triaged to MT despite CTAS scores of 2 or 3, possibly reflecting the impact of ED overcrowding. Only $1 / 3$ of study patients in the MT area were CTAS level 4 or 5 . There was a high rate of admission for these study patients $(27 \%)$. Age and female gender were identified as risk factors for falls and $50 \%$ of the elderly fall patients seen in our ED MT presented with fractures. Many seniors presenting to the ED with fall-related injuries have a history of prior falls suggesting "at risk" individuals could be identified and enrolled into a fall-prevention program for seniors.

Keywords: falls, elderly, injury

\section{P083}

Mass gathering medicine elective: emergency medicine education in the field 
J.E. Paul, BSc, MD, A. Lund, MD, A.S. Guy, R.K. Earle, BSc, S. Jamani Lund, BSN, MN, S.A. Turris, BHSc, MSN, PhD, Faculty of Medicine, University of British Columbia, Vancouver, BC

Innovation concept: High patient volumes are common at mass participation sporting events, concerts and music festivals. As highlighted at the 2013 Boston Marathon bombing, unanticipated events can result in a mass casualty incident, requiring on-site emergency medicine (EM) and disaster response. The literature contains sparse reference to learner participation in mass gathering medicine (MGM) or disaster medicine (DM). Limited resources and unpredictable conditions at mass gatherings help prepare medical learners for challenges faced in emergency departments, mass casualty incidents, and disasters. Methods: A longitudinal and shift-based elective for EM residents and medical students has run at UBC since 2008. Residents complete event shifts under attending physician supervision, with each event shift 'equivalent' to an EM shift. Set in exciting, dynamic and sometimes austere inter-professional environments, learners are exposed to management of unique, acute presentations in the prehospital setting, and involved in operational and logistical issues in MGM and DM. Bidirectional evaluation addresses learning objectives consistent with the Royal College of Physicians and Surgeons of Canada CanMeds Physician Competency Framework. Curriculum, tool, or material: From 2008 to 2013, 61 residents and 126 medical students have participated in 77 MGM events. Based on five years of post-event participant survey data, the responses have been overwhelmingly positive and learner participation has enriched medical teams at numerous mass gatherings. Conclusion: The MGM elective provides a platform for physician training, serving as an introduction to MGM, community service, leadership, hands-on field experience, inter-professional team collaboration, emergency management and disaster planning. Given the important skill-sets attainable and the lack of formal MGM and DM training options for residents, expansion of elective and possible fellowship opportunities benefit both learners and the community. In light of the inter-disciplinary nature of MGM, this elective structure could be readily incorporated into other medical and allied health care training programs such as nursing, physiotherapy, paramedicine, and chiropractic schools.

Keywords: innovations in EM education, mass gathering, disaster education

\section{P084}

In person crowdsourcing at an emergency medicine continuing education conference: an innovative teaching method R. Penciner, MD, MSc, University of Toronto, Toronto, ON

Introduction: Crowdsourcing is the practice of obtaining needed services, ideas or content by soliciting contributions from a large group of people and especially from an online community. Crowdsourcing has often been used to solicit opinions and solve problems. The purpose of this study was to describe and evaluate a novel teaching method at a traditional continuing education (CE) event using a "crowdsourcing technique". Methods: Emergency Medicine (EM) Update Europe was a 5 day conference consisting of 15 forty-five minute traditional CE presentations. Sixty-three physicians registered for the conference, with most $(n=57)$ from Canada. Prior to the start of the conference, registrants were contacted by email and invited to submit up to 3 problems, controversies or questions related to EM that they would like to have discussed during the conference. Each day of the conference, a 15 minute period was devoted to an open crowdsourcing discussion facili- tated by the course director (PI) using the list of rank-ordered topics submitted. Participants were asked to complete an anonymous paper survey after the last crowdsourcing activity. The survey consisted of a 15 item questionnaire exploring satisfaction and attitudes towards the activity (Kirkpatrick evaluation levels $1 \& 2$ ). In addition, quantitative observational data was collected on frequency of participation during each topic. Results: Twelve topics were discussed over 5 days. There were 45 registrants present for each topic (range 42-48; SD 1.83) with an average of 9 (SD 2.84) participants contributing to each conversation. Thirty-two unique individuals $(67 \%)$ contributed to at least one of the conversations. Thirty-nine participants out of 48 completed the survey (response rate $81 \%$ ). Most (79\%) believed that they had knowledge and/or expertise to share with their colleagues. During the crowdsourcing conversation, of the 39 respondents, $80 \%$ reported that they had contributed at least once. Most (77\%) enjoyed the activity and $74 \%$ found the crowdsourcing conversation valuable. Most (64\%) reported that they often or very often trusted the opinions of those speaking during the activity. Over half (56\%) the respondents reported that they learned something during the crowdsourcing activity. Conclusion: Deliberate crowdsourcing is perceived as a worthwhile teaching method at an EM CE conference.

Keywords: crowdsourcing, CE, evaluation

\section{P085}

Pain and symptom control of renal colic patients presenting to regional emergency departments with and without a $\mathrm{CT}$ scanner N. Rafo, BSc, N. Argintaru, BSc, K. Milne, MD, M. Bhimani, MSc, MD, D. Grushka, MD, University of Western Ontario, London, ON

Introduction: Renal colic is a common Emergency Department (ED) presentation. The use of non-contrast Computer Tomography (CT) in diagnosing renal colic has increased and is the gold standard despite issues with costs, accessibility and potential radiation exposure. Although CT prevalence is increasing, many rural EDs do not have access to CTs on site. This study evaluated how the presence of onsite CT scanners in community ED's correlates with the pharmacological management renal colic Methods: A retrospective chart review was conducted at five regional hospitals: three with a CT scanner, and two without. 200 adult ED renal colic patients were selected per site in reverse chronological order. Data extracted included demographics, past medical history, imaging (ED and outpatient), analgesia and follow-up. Results: Of 994 patients included in the analysis, 400 (40.2\%) were from sites without a CT and 594 (59.8\%) were from sites with a CT. Although $82.5 \%$ of all patients presenting with renal colic received analgesia, the rate was significantly higher in ED with a CT on site $(85.2 \%$ vs. $78.5 \%, p<0.01)$. Nearly $60 \%$ of patients in sites with a CT received Opioids versus $45.5 \%(\mathrm{p}<0.01)$ in the other two sites. There was no significant difference in NSAID or antiemetic use in sites with or without a CT scanner. Interestingly, although the overall prevalence of alpha blocker use for renal colic management in the ED is $3 \%$, it was used significantly more in sites without a CT scanner ( $\mathrm{p}=0.04)$. Conclusion: Although different imaging rates are expected based on the availability of a CT scanner, there was a significant increase in the rate of analgesia when a CT was available on site. This difference is largely accounted for by increased rate of opioid use. The role of CT scans in the ED should be further investigated to identify whether patient-centered outcomes change in acute presentations of renal colic.

Keywords: renal colic, analgesia, rural 
P086

FOAMSearch: a search engine optimized for emergency medicine physicians

T. Raine, MD, T.M. Chan, MD, B. Thoma, MD, MA, M. Lin, MD, University of British Columbia, Vancouver, BC

Innovation concept: The Free Open Access Meducation (FOAM) movement has resulted in a rapid growth of openly accessible resources for Emergency Medicine and Critical Care (EMCC) practitioners and learners. Major search engines use algorithms that do not make it easy to find these resources because they are outranked by high traffic sites that are often commercial, pay-for-use, or of unknown provenance. FOAMSearch.net is an online portal that hosts a customized search engine which curates educational material for emergency physicians. Methods: The FOAMSearch.net Custom Search Engine examines a curated list of websites to find results that are highly relevant to emergency physicians. A list of trustworthy open access blogs and podcasts was generated by reviewing a comprehensive list compiled at LifeInTheFastlane.com. Websites on this list as well as knowledge translation and decision support tools were included if they had identifiable authors, contained primarily original content, and were recommended by at least one other member of the FOAM community. They were excluded if the content was primarily re-posting of other sites' content. The websites of prominent emergency medicine and critical care journals were also added. Curriculum, tool, or material: FOAMSearch.net provides a Google custom search engine (CSE) limited to sites, tools, and journals known and trusted by the FOAM community. It provides EMCC providers, educators, and researchers a simple way to filter Google searches to find the most relevant content. It is not meant to provide a comprehensive search, but to help medical education resources that are relevant to EMCC providers to be found quickly and precisely Conclusion: FOAMSearch.net represents a disruption of 'traditional' web searches that can help to change the landscape of medical education by narrowing the search field to a regularly updated list of trusted resources.

Keywords: innovations in EM education, search engine, open access

\section{P087}

An exploration of the consequences of 3 rd year clerkship clinical assessments

T. Robertson, BMSc, A. Murnaghan, MD, J. Frank, MD MA(Ed), K. Moreau, PhD, University of Ottawa, Department of Emergency Medicine, Ottawa, ON

Introduction: To determine whether the results from assessments should be supported or refuted, medical education researchers have suggested that the validity framework of the Standards for Educational and Psychological Testing be used. The fifth source of validity evidence is consequential evidence which is concerned with the impact as well as the intended and unintended consequences of assessments. This study describes the fourth year medical students' perspectives on the consequences of third year clerkship assessments, including the daily encounter cards (DECs) that are used during the emergency medicine rotation. Consequences include how assessments impact student learning and clinical practice, how assessments are used to prepare students for future licensing exams and residency applications, and the students' perceptions on the burdens and benefits of assessments. Methods: Purposeful sampling was performed and 13 University of Ottawa fourth year medical students were recruited and interviewed using a semi-structured interview framework. An iterative coding process was used and data was coded into recurrent themes. Results: From the analysis, several themes emerged regarding the impact of assessments on students' learning and clinical practice: a) helps to develop their clinical approach, b) identifies areas of strength and weakness, and c) provides feedback on their progress in comparison to peers. There was a general theme that assessments did not prepare students for future licensing exams, with the exception of the objective structured clinical examinations (OSCEs). Students identified that assessments had direct and indirect impacts on their residency applications. Themes that emerged regarding the benefits and burdens of assessments varied according to the type of assessment but were related to: a) clinical relevance, b) preceptor dependence, and c) variability in assessment execution. Conclusion: This research offers valuable insight into medical students' views on the consequences of assessments. Incorporating these views into the development, choice and implementation of clinical assessments used during clerkship can potentially improve their validity and optimize students' learning experiences during their core emergency medicine rotation.

Keywords: medical education, clinical assessments, clerkship

\section{P088}

Inside the brain of an expert: measuring cognitive load in emergency medicine physicians

N. Roth, BSc, A. Szulewski, MD, D. Howes, MD FRCPC, Queen's University School of Medicine, Kingston, ON

Introduction: Task evoked changes in pupil size have been shown to correlate with cognitive processing demands. The magnitude of this change has been found to be a reliable marker of cognitive load in properly controlled settings. The purpose of this study was to use task-evoked pupillary responses (TEPRs) to compare patterns of cognitive load between novices and more expert physicians answering clinical emergency medicine questions of varying difficulty. Methods: Twenty participants were recruited into two experimental groups based on level of medical training - novice $(\mathrm{n}=10$; junior medical students) and expert ( $\mathrm{n}=10$; PGY-4, PGY-5 and first year attending emergency physicians). Tobiic mobile eye-tracking glasses were used to assess pupil diameter change from baseline as participants answered standardized clinical emergency medicine questions in a controlled setting. Questions were categorized by difficulty a priori. Results: The TEPRs behaved in a manner suggesting good correlation with cognitive load. The TEPR was significantly higher in the novice group compared to the expert group $(p<0.001)$. TEPR was significantly greater for difficult questions posed to experts compared to easy questions $(\mathrm{p}=0.001)$ and for incorrect compared to correct questions $(\mathrm{p}=0.002$ ). Novice TEPR patterns were similar for both easy and difficult questions $(\mathrm{p}=0.815)$. Variability in TEPR was also greater for novices compared to experts $(\mathrm{p}<0.001)$. These results suggest that more experienced physicians are less cognitively taxed by medical knowledge demands. Conclusion: TEPRs show systematic and quantitative differences between novices and experts suggesting strong correlation with cognitive loading. TEPRs may be able to quantitatively differentiate levels of medical expertise, and have the potential to provide the basis for a new, more objective assessment of medical competence.

Keywords: cognitive load, clinical decision making, medical education

\section{P089}

Action plans in patients presenting to emergency departments with asthma exacerbations 
E. Cross, M. Bhutani, MD, S.R. Majumdar, MD, R.J. Rosychuk, PhD, C. McCabe, PhD, E. Lang, MD, R. Leigh, MD, C. Villa-Roel, B.H. Rowe, MD, MSc, University of Alberta, Edmonton, AB

Introduction: Written asthma action plans (AAP) are associated with reduced Emergency Department (ED) visits and hospitalizations due to exacerbations, as well as improved medication adherence. This study describes the knowledge and use of AAP by patients presenting with acute asthma to three urban Canadian EDs. Methods: Secondary analysis of data obtained from two randomized controlled trials of patients discharged from the ED with acute asthma. Data were collected through ED interviews and chart review. Descriptive analyses are reported using proportions and medians with interquartile range (IQR); bivariable comparisons were performed. Results: From 176 participants, the median age was 27 years (IQR: 23,29) and 97 (55\%) were female. Overall, 42 (24\%) patients reported having an AAP; however, only $6(14 \%)$ were written. Five $(3 \%)$ patients reportedly used "home remedies" only. Those with an AAP were more likely to have received asthma education in the past $(21 \%$ vs. $8 \%, p<0.004)$. More patients with an AAP reported having been to an ED in the past for acute asthma than those without an AAP $(95 \%$ vs. $74 \%, \mathrm{p}=0.003)$ and were also more likely to be on inhaled corticosteroids (ICS) or ICS combination agents at presentation $(81 \%$ vs. $55 \%$, p $<0.005)$. Conclusion: Despite good evidence for their use, uptake of written AAPs remains very low in patients presenting to ED with acute asthma. Most AAPs were verbal, and the subsequent actions taken were often unsupported and ineffective. AAP use is an important issue and methods to enhance AAP uptake and adherence are urgently needed.

Keywords: asthma, emergency medicine

P090

Good to the last drop: a case of an entrapped tongue

M. Rubin, MD, Westchester Medical Center, New York Medical College, NY

Introduction: A simple news search reveals multiple accounts of children managing to get their tongues stuck in glass bottles. Often in an attempt to suck out the very last drop, a child will stick their tongue into the bottleneck thereby creating a vacuum inside the bottle, which further sucks their tongue into the bottle. A 7-year-old child was transferred to our pediatric emergency department after having his tongue entrapped in the neck of a standard glass pop bottle for nearly 4 hours. The tongue, visible through the class appeared swollen and ischemic. Methods: The initial concern was airway preservation. If the glass shattered, there was risk for massive bleeding and subsequent airway compromise. Early attempts were made to pass a naso-gastric tube into the bottle and behind the tongue in order to break the vacuum seal. Other considerations included drilling a hole into the bottom of the bottle but not until a definite airway could be secured. Results: Naso-tracheal intubation was performed to protect the airway prior to attempting any aggressive techniques. The patient was brought to the OR where an ear, nose and throat surgeon was able to clamp the tongue and successfully remove the bottle. The patient remained intubated for five days follow the procedure due to extensive edema. Conclusion: Airway should always be the first consideration when addressing a situation such as a tongue entrapped in a bottle. If aggressive measures were attempted prior to securing the airway there could have been massive bleeding and airway compromise. Intubating such a patient is prudent move in order to reduce the likelihood of devastating outcomes.
Keywords: airway, pediatrics, tongue

P091

Mass casualty event simulation; preparing Boston's first responders for disaster scenarios post Boston Marathon Bombings M. Rubin, MD, Beth Israel Deaconess Medical Center, Harvard Medical School, Boston, MA

Introduction: The explosion at the finish line of the Boston Marathon killed three people and critically injured over 20 others. It is believed that the timely response by medically trained personnel with the use of tourniquets is responsible for saving multiple lives. As a large scale training exercise, The Harvard Disaster Medicine Fellowship organized a mass casualty disaster simulation with similar injury patterns to the recent Boston Marathon Bombings. Methods: The simulation was held at military base that was designed to mimic an urban environment. The drill involved multiple local agencies including Fire, Police, National Guard, the Boston Medical Reserve Corp and all the major EMS services from the Boston area as well as physicians and nurses from Beth Israel Deaconess Medical Center. Ninety-three simulated patients were "moulaged" with predetermined injuries and given extensive instruction on how to roll play during the simulation. The scenario began with a lone shooter injuring 16 victims and was then further complicated by a secondary explosion once the initial first responders had arrived on scene. With a total of nearly 100 simulated victims, all agencies had to work together to triage, treat and transport large numbers of critically injured patients. Results: Initial START triage classifications were correlated to each patient's pre-determined injuries. Patients with major bleeding who did not receive appropriate hemorrhage control were instructed to decompensate which resulted in three "immediate" classified patients progressing to "expectant." With only few exceptions, the patients were transported to hospital appropriately by severity classification in a timely fashion. Conclusion: Large-scale multi-agency simulation is an effective means of identifying deficiencies in preparedness and applying lessons learned to better respond to future disaster scenarios. This simulation re-enforced the benefits of early hemorrhage control and the need for real time problem solving during any mass casualty event.

Keywords: disaster medicine, mass casualty, simulation

\section{P092}

Ultrasound, an underutilized available resource in one Tacloban hospital

M. Rubin, MD, Harvard Medical School, Boston, MA

Introduction: Typhoon Haiyan devastated the city of Tacloban, the capital and medical epicenter of the province of Leyte Philippines. With an estimated 26000 injured individuals in the region, hospitals in the Tacloban area attempted to restore functionality as quickly as possible. Many of the Tacloban hospitals began to treat patients despite severely limited medical capabilities. The RTR Hospital in Tacloban opened their ED and began admitting patients despite the lack of laboratory, radiological, intensive care or surgical capabilities. While taking inventory of the hospital's radiology department a functional ultrasound machine was discovered. The ultrasound machine was brought to the ED to be used for the identification of patients with abdominal pathology who would benefit from transfer to a hospital with surgical capabilities. Methods: Local physicians staffing the ED were given a brief introduction and instruction on how to perform a FAST exam. They 
were encouraged to scan all patient for whom they were considering surgical intervention. Results: Local doctors who had no previous experience with ultrasound did not see the benefit of using the ultrasound and preferred to rely only on clinical exam. Conclusion: Ultrasound is a low maintenance, harmless, portable tool that can reveal much information in the hands of a skilled operator. In a disaster setting where there is no access to x-ray, CT scan or MRI, ultrasound may be the only available tool with which to identify occult pathology that alters management. Despite initial resistance, providers should be educated, mentored and encouraged to use ultrasound as a diagnostic tool.

Keywords: ultrasound, disaster medicine, FAST exam

\section{P093}

\section{An outbreak of ciguatera fish poisoning in New York City}

M. Rubin, MD, Beth Israel Deaconess Medical Center, Harvard Medical School, Boston, MA

Introduction: A 52-year-old female was brought to the emergency department (ED) accompanied by her husband and two grown children. She presented with an acute, unexplained onset of neurological symptoms associated with vomiting. Within one hour of evaluating the woman each family member in turn became acutely ill requiring supportive care. Each family member experienced the acute onset of cramps, nausea, weakness, and paradoxical dysesthesias. The patient's husband, age 55, experienced an abrupt onset of extreme bradycardia and hypotension requiring aggressive fluid resuscitation. On history all members had consumed barracuda prior to presenting to the ED. Methods: Each patient's hospital record was reviewed and symptoms and clinical findings compared with those expected in ciguatera poisoning. Results: Given the recent ingestion of barracuda and pathognomonic presentation, a ciguatera outbreak was diagnosed and reported to the Department of Health. All patients improved with supportive therapy. Conclusion: Ciguatera fish poisoning results from the consumption of large, predatory, tropical fish that have bioaccumulated ciguatoxins. The poisoning manifests with gastrointestinal, cardiovascular and neurological symptoms. Ciguatera is an underreported illness. It is often difficult to identify in isolated cases as it is not prevalent in the northeastern USA. Early identification of the poisoning based on history and clinical findings is important from a public health perspective in terms of preventing further outbreaks.

Keywords: outbreak, ciguatera, public health

\section{P094}

Simulation saves a life in a small community emergency department; a model to simulate ultrasound guided pericardiocentesis M. Rubin, MD, Beth Israel Deaconess Medical Center, Harvard Medical School, Boston, MA

Introduction: When was the last time you stuck a needle in someone's heart? Performing a pericardiocentesis under ultrasound guidance has become the standard of care and many physicians lack experience in performing the procedure. In order to become familiar with the procedure in a small community emergency department, we developed a simulation model in order to practice. Methods: With guidance from previous simulation literature a model was created using food grade gelatin, balloons, dyes, and a molding in the shape of a human thorax. One balloon was inserted into a second and filled with $300 \mathrm{cc}$ of water with blue dye and tied. The outside balloon was then filled with an additional $200 \mathrm{cc}$ of red dye and tied closed. The concentric balloons represent a simulated heart with an effusion and were placed anatomically into the thorax molding and set in an opaque gelatin. Results: When ultrasound was applied to the model the resulting images were similar to that of a patient with a cardiac effusion. Physicians were able to use ultrasound guidance to perform a simulated sub-xyphoid pericardiocentesis on the model. The needle could be visualized entering the pericardium where the red fluid could be aspirated; if the inner balloon were pierced the aspirate would be blue indicating a perforation of the myocardium. Conclusion: Practicing on simulated patients helps build confidence and perfect technique. Having to perform a pericardiocentesis in a small community ED is not a common occurrence. Having recently simulated the procedure enabled one provider to effectively perform the procedure in the $\mathrm{ED}$, allowing for successful resuscitation of a patient with cardiovascular collapse secondary to pericardial tamponade.

Keywords: pericardiocentesis, ultrasound, simulation

\section{P095}

Is there a role for ultrasound in the collecting area of a disaster zone?

M. Rubin, MD, Beth Israel Deaconess Medical Center, Harvard Medical School, Boston, MA

Introduction: The explosion at the finish line of the Boston Marathon killed three people and critically injured over 20 others. It is believed that the timely response by medically trained personnel and the proximity to multiple tertiary care hospitals was responsible for saving many lives. As a large scale training exercise, The Harvard Disaster Medicine Fellowship organized a mass casualty disaster simulation with similar injury patterns to the recent Boston Marathon Bombings in an area with only a single tertiary care facility. Methods: The simulation mimicked an explosion in a populated urban environment. The drill involved multiple local agencies including Fire, Police, National Guard, and the major EMS services in the Boston area as well as physicians and nurses from Beth Israel Deaconess Medical Center. Ninety-three simulated patients were "moulaged" with predetermined injuries and given extensive instruction on how to roll-play during the simulation. Multiple simulated patients were pre-selected to have occult abdominal trauma and given extensive instruction to decompensate at different points during the drill. Results: Patients were triaged using the START method and then relocated to three collecting zones by triage designation. The treatment officer assigned patients for transport to one of four available receiving hospitals. One trauma center, one level two hospital and two small community facilities with limited surgical capability. Most of the designated patients were initially triaged appropriately, but had to be re-triaged once they became unstable. Conclusion: Patient's with "immediate" (red) designation were always transported to highest level of care available. Multiple patients who presented as "delayed" (yellow) but had occult injury were transferred to inappropriate facilities. Given that most patients remained in the collecting area for no less than 5 minutes, there would have been ample time to perform a focused assessment with sonography for trauma (FAST) exam. In a real casualty situation, a positive FAST exam can herald an impending decline in stability and prompt re-triage and appropriate hospital assignment. Future study should involve using an ultrasound in the treatment area to further stratify patients and identify occult injury.

Keywords: ultrasound, simulation, disaster medicine 
P096

Capacity planning in the emergency department using computer simulation to evaluate future patient visits D.W. Savage, PhD, D. Woolford, PhD, B. Weaver, MSc, D. Wood, MD, Northern Ontario School of Medicine, Greater Sudbury, ON

Introduction: Capacity planning is a process for evaluating the demand for resources and determining production capacity requirements. In an emergency department (ED), this process might look at the number of required treatment areas, imaging machines or the size of laboratory facilities. In Thunder Bay, Ontario the number of patient visits to the ED has increased from 1.5 to $6 \%$ per year over the past 8 years. Capacity planning can be used to assess existing facilities to determine their ability to handle an increase in demand. We propose to perform a capacity planning exercise for the Thunder Bay ED using computer simulation modelling to assess the effect of expected increases in patient visits. Methods: We first used linear regression and the total number of patient visits for 8 years of data (i.e., all of the existing data for a relatively new ED) to forecast the number of patient visits in 2017 and 2022. Physician staffing levels were predicted for those years according to the hours of coverage model used in Ontario for ED staffing. We then developed a computer model that simulates the major processes patients experience while visiting the ED including: triage, physician initial assessment, laboratory testing, diagnostic imaging, specialist consultation and final disposition. The model focuses on acute patients (i.e., primarily CTAS 1,2 , and 3 ). We then ran the model with the number of patients forecasted in 2017 and 2022 and observed the simulated patient wait times and length of stay given increased number of visits without an increase in the number of stretchers. Finally, we increased the number of stretchers incrementally to determine how much treatment space will be required in the future to meet patient demand. Results: The forecasted patient visits to the ED increased from 2012 by $16.4 \%$ and $32.5 \%$ for the years 2017 and 2022, respectively. The simulation model predicted an average length of stay (LOS) in 2012 of 320 minutes (current ED configuration), 459 minutes in 2017, and 4275 minutes in 2022. An additional 5 stretchers in 2022 will reduce the LOS to 265 minutes. Conclusion: Capacity planning has great potential to assess the ED resources that may be required in the future. The probable increase in patient visits by 2022 will require greater stretcher resources to ensure patient flow. However, the required increase in stretchers will likely be feasible for ED managers.

Keywords: capacity planning, simulation

P097

Information needs and preferences of healthcare providers and healthcare consumers in $\mathbf{3 2}$ general emergency departments in Canada: findings from the Translating Emergency Knowledge for Kids (TREKK) Knowledge Mobilization Initiative

S. Scott, PhD, L.M. Given, PhD, L. Albrecht, MEd, L. Hartling, PhD, D. Johnson, MD MSc, M. Jabbour, MD, T. Klassen, MD, MSc, University of Alberta, Edmonton, $\mathrm{AB}$

Introduction: In Canada, the majority of children requiring emergency care are treated in general emergency departments (EDs). The Translating Emergency Knowledge for Kids (TREKK) initiative is a multi-phase, pan-Canadian project aimed at ensuring the latest research in pediatric emergency medicine is implemented in general EDs. To partner with 32 general EDs to determine knowledge needs and preferences of ED health professionals (HCP) and parents seeking care for their children (consumers). Methods: A mixed methods approach was used. Two electronic surveys were developed to collect asynchronous survey data via an interactive iPad 'app', which was analyzed using SPSS. Next, 7 EDs were purposefully sampled to participate in qualitative focus groups and observations. Results: Data collection occurred from May 2012 to October 2013 and resulted in 1,768 HCP and 1,099 consumer surveys. HCP responses showed that while $82 \%$ currently find information needed to provide care to children in the ED by talking to colleagues, they would prefer to find information via professional development opportunities ( $80 \%$ ) and printed summaries $(63 \%)$. Also indicated were current information needs to care for ill children, including: protocols and accepted treatments for common conditions (68\%), evidence-based clinical pathways and CPGs (66\%), and evidence-based information on new diagnoses and treatments $(61 \%)$. Health professionals also indicated that they wanted more pediatric clinical information on multi-system trauma (49\%), severe head injury $(43 \%)$, and meningitis (39\%). Consumer responses revealed that $39 \%$ had sought health information prior to coming to the ED. Consumers typically found health information by talking to trusted professionals $(69 \%)$ or through internet search engines $(53 \%)$; however, $74 \%$ indicated that they would prefer to learn health information by talking inperson with a healthcare professional. Parents reported additional information is needed about being in the ED with their child, including explanation of the child's illness/condition (47\%), treatment information (44\%), and care instructions (42\%). Conclusion: The findings from this study will be of value to multiple stakeholders and will enable the TREKK Knowledge Mobilization Initiative to offer evidence-based information and interventions to ensure the best quality care for all Canadian children.

Keywords: pediatrics, knowledge needs

\section{P098}

Treatment of pediatric diabetic ketoacidosis in Canada: a review of treatment protocols utilized in Canadian pediatric emergency departments

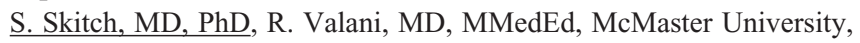
Hamilton, ON

Introduction: Diabetes is the most common pediatric endocrine disorder, and diabetic ketoacidosis is the leading cause of diabetes related morbidity and mortality. There is no national treatment protocol for the emergency management of pediatric diabetic ketoacidosis (pDKA) patients in Canada. Rather there are a variety of local protocols that vary between different provinces, health authorities, and intuitions. To complicate this further, the international guidelines are also variable in certain management aspects. The purpose of this study was to identify the differences in management of pDKA and use this as an impetus to align treatment protocols, prevent conflicting views, and provide guidance for practicing emergency physicians. Methods: We reviewed the treatment protocols for the management of pDKA in the emergency department from 11 pediatric tertiary care centers across Canada. Furthermore, one provincial and 4 international guidelines were also reviewed in order to identify specific management steps. For each treatment protocol, the following recommendations were reviewed: initial fluid bolus, maintenance fluid rate and composition, insulin infusion rate, potassium replacement, monitoring and adjustment for serum glucose, administration of bicarbonate, and treatment for cerebral edema. Results: Responses were obtained from all eleven sites contacted. Treatment guidelines were consistent in 
their recommendations regarding timing and dosage of intravenous insulin, potassium replacement, and monitoring and adjustment for serum glucose. The main areas of variability between treatment protocols regard recommended initial fluid boluses volumes (range: 5$20 \mathrm{cc} / \mathrm{kg}$ ) and rates (over 20 to 120 minutes), maintenance fluid rates (either based on weight or a $48 \mathrm{hr}$ deficit), and bicarbonate administration. The majority of treatment protocols have specific recommendations for the management of cerebral edema. Conclusion: This study is the first national review of treatment protocols for pDKA utilized in Canada. There are many areas of commonality and consensus between the different treatment approaches, with specific differences in fluid bolus management, maintenance, and bicarbonate administration. Further studies and collaboration are necessary to develop national guidelines consistent with best evidence practice to guide the emergency room management of pDKA.

Keywords: pediatric emergency medicine, diabetes, guidelines

\section{P099}

A retrospective review of time-to-analgesia for the direct from scene trauma patients transported to a level-1 trauma centre by a single EMS provider

A. Slomer, MD, M. McGowan, MHK, K. Nixon, MSc, D. MacKinnon, $\mathrm{MD}$, University of Toronto, Toronto, ON

Introduction: Providing timely and adequate analgesia is a challenging aspect of the care of patients with acute traumatic injuries. We sought to describe time from first contact with a care provider and administration of analgesia in direct from scene trauma patients. Methods: A six-month retrospective chart review (January - June 2012) of trauma team activations (TTAs) who were GCS $>12$ and transported direct from scene by a single municipal EMS provider to a level-1 trauma center was conducted. Exclusions were intubation (prehospital or upon arrival), VSA, and without an Ambulance Call Record in the chart. Clinical, demographic, and administrative data was extracted with $10 \%$ independently reviewed for inter-rater reliability. Results: 187 TTAs were identified; 70 direct from scene and 51 met inclusion criteria. Only 2 of 51 patients (4\%) received pain medication prehospital, while $37(73 \%)$ received it upon arrival to the trauma room with similar time-to-analgesia irrespective of type or area of injury: penetrating-extremities [4 $(80 \%), 14 \pm 8 \mathrm{~min}$ ]; penetrating-other [11 (58\%), 14 $\pm 12 \mathrm{~min}]$; blunt-extremities [10 (91\%), $15 \pm 9 \mathrm{~min}]$; blunt-other [12 (75\%) $12 \pm 8 \mathrm{~min}] .59 \%(30 / 51)$ of these patients had IV access established prehospital. Time-to-analgesia from first contact with care provider for the 35 patients who did not receive any prehospital relief was $40 \pm 14 \mathrm{~min}$, while those 2 prehospital was $26 \pm 9 \mathrm{~min}$. Conclusion: This review suggests that the current prehospital analgesia directive may be too restrictive, given that the majority of TTA patients were determined to be appropriate for analgesia upon arrival to the trauma bay. With over half of TTAs arriving with established IV access, opportunities may exist to improve the trauma patient experience by providing more timely pain relief in the prehospital setting.

Keywords: pre-hospital care, trauma, pain management

P100

Corticosteroid use in the treatment of anaphylaxis in the emergency department

V. Smith, MD, M. Klingel, MSc, S.L. McLeod, MSc, E. Loubani, MD, University of Western Ontario, London, ON
Introduction: Emergency department (ED) treatment of an acute anaphylactic episode should consider the possibility of biphasic reactions, which can develop within 72 hours of the initial allergic reaction. Although corticosteroids have been suggested for prevention of biphasic or multiphasic anaphylaxis, there is no clear consensus regarding their use. The objective of this study was to determine the proportion of patients treated with corticosteroids after presenting to the ED with acute anaphylaxis and determine the proportion that had a biphasic reaction within 72 hours of their initial ED visit. Methods: This was a retrospective medical record review of all patients presenting to one of two tertiary care EDs (annual census 125,000) with a discharge diagnosis of anaphylaxis or anaphylactic shock over a one year study period (April 2012-March 2013). Patient demographics, medications administered, corticosteroid use, admissions and in-hospital deaths were recorded by trained research personnel. Results: Of the 140 patient encounters included, 118 (84.3\%) had epinephrine administered either pre-hospital or in-hospital. Mean (SD) age was 22.3 (17.5) years and $76(54.3 \%)$ were male. Median (IQR) time to epinephrine administration was $11 \mathrm{~min}(1,24) .127(90.7 \%)$ patients received corticosteroids in the ED. Of these, $40(31.5 \%)$ were given orally and 87 $(68.5 \%)$ were given intravenously. Median (IQR) time to steroid administration was $36.5 \min (19,57) .85(60.7 \%)$ patients received antihistamine, and $101(72.1 \%)$ received diphenhydramine. 5 (3.6\%) patients were admitted. Median (IQR) ED length of stay was 253.5 $\min (201.5,314)$. Treatment patterns were similar between adult and paediatric patients. There were no in-hospital patient deaths. Biphasic reactions occurred in $6(4.3 \%)$ patients. All patients with biphasic reactions were given corticosteroids during the initial ED presentation. Conclusion: Despite the paucity of evidence supporting their use in the prevention of biphasic anaphylactic reactions, corticosteroids were used by the majority of clinicians in the treatment of anaphylaxis in the ED. Future studies should assess the use of corticosteroids to prevent biphasic reactions in anaphylaxis.

Keywords: anaphylaxis, corticosteroid, biphasic reaction

\section{P101}

Impact of the age of stored blood on trauma patient mortality: a systematic review

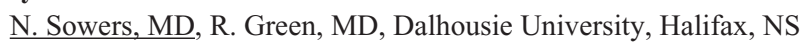

Introduction: The impact of the age of stored packed red blood cells (pRBCs) on mortality in trauma patients requiring transfusion is unknown. The objective of this systematic review is to identify and describe the available literature on the use of "new" versus "old" blood in the trauma patient population. Methods: A systematic search of MEDLINE, EMBASE, Lilac, and the Cochrane Database was conducted. Studies were included in the review if they compared the transfusion of "new" blood versus "older" blood in adults ( $>18$ years) that sustained a traumatic injury, as defined by the investigator. Studies were considered relevant for the purpose of this study if they included patients with traumatic injury who were transfused pRBCs, described the age of blood, and reported mortality as an outcome. All variables were extracted using a standardized data-capture form, and two investigators independently reviewed papers. Results: The systematic review yielded a total of seven studies, including a total 5,586 patients. The seven included studies were heterogeneous in inclusion criteria, number of units of pRBCs transfused, and definition of "new" versus "old" blood, and therefore a meta-analysis was not performed. Four of the studies indicated that older blood was associated with an increase in 
patient mortality with a range of odds ratios from 1.12 to 4.0 . Two studies failed to show statistical significance but concluded no increase in mortality with older blood while one study suggested a potential benefit to older blood with an OR 0.959. Conclusion: The impact of the age of blood on the outcome of trauma patients is inconclusive. Further investigation is warranted to describe this effect, as well as establish an objective standard of what constitutes "old" blood.

Keywords: trauma, transfusion

\section{P102}

Evaluation of a point-of-care ultrasound scan list in the emergency department at Black Lion Hospital, Addis Ababa, Ethiopia M.R. Stachura, MD, M. Landes, MD, MSc, R. Venugopal, MD, MPH, F. Aklilu, PhD, MD, J. Sarrazin, MD, A. Azazh, MD, Vancouver General Hospital, Vancouver, BC

Introduction: Emergency departments in low to middle income countries often have limited diagnostic imaging capabilities. Point-of-care ultrasound is a relatively inexpensive and highly effective technology used commonly by emergency practitioners in most high-income countries. The goal of this study was to assess the impact of point-of-care ultrasound in the evaluation and management of patients presenting to an urban emergency department in Ethiopia. Methods: Patients presenting to the emergency department at Black Lion Hospital in Addis Ababa, Ethiopia between February 2012 and February 2013 were eligible for inclusion into this prospective cohort study. Physicians responsible for the care of emergency department patients were provided a list of 15 point-of-care ultrasound scans and were asked to refer patients when a scan was clinically indicated. Patient or next of kin consent was obtained. Ultrasound scans were performed and interpreted at the bedside in real-time, by a qualified emergency department ultrasonographer. The treating physician completed a data collection form including demographic and clinical details and evaluated the impact of ultrasound findings on patient management. Results: A total of 118 patients were enrolled. The average age was 35 years and $42.5 \%$ of patients were female. Ultrasound scans were performed for one or more of the following indications: assessment of trauma (43.2\%), medical shock/volume status $(24.6 \%)$, dyspnea $(20.3 \%)$, abdominal pain $(9.3 \%)$, procedure guidance $(7.6 \%)$, musculoskeletal $(5.1 \%)$, pregnancy $(3.4 \%)$, deep vein thrombosis $(3.4 \%)$, renal/bladder $(2.5 \%)$, cardiac arrest $(1.7 \%)$, and other $(2.5 \%)$. The most common scans performed in the emergency department were: pericardium $(78 / 118$ patients $=66.1 \%)$, abdominal free fluid (61.9\%), pleural effusion/hemothorax (43.2\%), IVC (36.4\%), pneumothorax (32.2\%), and global cardiac activity (21.2\%). Overall, ultrasound was found to provide clinically useful information in $94.9 \%$ of patients scanned. In $44.9 \%$ of patients, ultrasound findings changed patient management plans by either changing the working diagnosis $(27.1 \%)$, resulting in a new treatment intervention (23.7\%), resulting in a procedure/surgical intervention (14.4\%) leading to consultation with a specialist (13.6\%), or changing a disposition decision (7.6\%). For those scans where it did not change management, ultrasound was still judged to provide clinically useful information such as narrowing the differential diagnosis, excluding a life threatening diagnosis, or providing diagnostic certainty. Conclusion: In this Ethiopian emergency department setting, point-of-care ultrasound had a significant impact on diagnosis and management plans for emergency department patients, particularly those presenting with polytrauma and undifferentiated shock. In the future, these results can be used to develop a locally relevant emergency department ultrasound curriculum.
Keywords: emergency department ultrasound, international, point-ofcare ultrasound

\section{P103}

The effectiveness of a student volunteer-based research assistant program in a pediatric emergency department

P.E. Steadman, MSc, J. Crudden, T. Naranian, J. Oliveria, K. Boutis, MD, MSc, University of Toronto, Toronto, ON

Introduction: Emergency Department (ED) student-based research assistant programs have been shown to be effective in enrolling patients when the students receive university course credit or pay. However, the impact of utilizing student volunteers on research outcomes is relatively unknown. The main objective of this study was to determine how often potentially eligible children were accurately identified by volunteer research assistants for enrollment into prospective research in the ED. We also examined the frequency of successful enrollments and the accuracy of data capture. Methods: This was a prospective cross-sectional study of student volunteer research assistant performance in a tertiary care pediatric ED between March 2011 and July 2013. A student participant's primary role was to screen and facilitate enrollment of ED patients into clinical research. For each volunteer, we recorded demographics, number of screenings, enrollments, and data capture accuracy. Results: Over five six month sessions, 151 student volunteers participated. Of these, $77.3 \%$ were female, $58.8 \%$ were undergraduate students, and $61.1 \%$ were interested in medical school. Student volunteers accurately screened 11,362/13,067 (87.0\%) children, and they accurately identified 4,407/4,984 (88.4\%) potentially eligible children for study enrollment. Of the 3,805 eligible for enrollment exclusively by the student volunteers, 3,228 (84.8\%) families/children consented and completed all study procedures. Furthermore, student volunteers correctly entered 11,660 /12,567 (92.8\%) data points. Conclusion: Utilizing student volunteers to facilitate research enrollment in the ED is effective and allows for the capture of a high percentage of potentially eligible patients into prospective clinical research studies.

Keywords: research, data collection, education

\section{P104}

The professional benefits for volunteer research assistants in a pediatric emergency department

P.E. Steadman, MSc, J. Crudden, T. Naranian, J. Oliveria, K. Boutis, $\mathrm{MD}, \mathrm{MSc}$, University of Toronto, Toronto, ON

Introduction: Some emergency departments (ED) utilize university student volunteers to facilitate enrollment of patients into prospective studies; however, the impact of this experience on participant careers is relatively unknown. We determined the proportion of successful post-graduate school/research job applications supported by our program reference letter. We also examined participant satisfaction. Methods: This was a prospective cohort study of volunteer research assistants in a tertiary care pediatric ED from September 2011 to July 2013. Students volunteered one shift per week for at least six months. They also completed three surveys: (1) Entrance - recorded demographics and goals for entering the ED research assistant program; (2) Exit - included information on satisfaction with program, reasons for leaving the program, and future career goals; (3) Follow up - every six months a survey and individual e-mails were sent to record positions secured since leaving the program. Results: There was a total of 920 university student applicants 
for 127 volunteer positions over the study period. Of these, $77.3 \%$ were female and $58.8 \%$ were undergraduate students. Of those with follow-up information, 89/101 (88.9\%) obtained school/research positions supported by our program reference letter. Further, $80.7 \%$ ranked their satisfaction with the program at least a seven on a 10 point categorical scale, and $82.5 \%$ reported that they "agreed/strongly agreed" that the research assistant program helped with their career goals. Conclusion: A volunteer student program is in high demand for university students interested in health sciences/research and has a potentially beneficial career impact for its participants.

Keywords: Funding, Volunteer, Hospital volunteers

\section{P105}

Utility of a validated prediction model for diagnosing acute heart failure -results of a prospective trial

B. Steinhart, MD, P. Levy, MD, G. Moe, MD, M. McGowan, MHK, H. Vandenberghe, PhD, D. Clark, MD, G. Devlin, MD, C. Mazer, MD, Saint Michael's Hospital, Toronto, ON

Introduction: Diagnosing undifferentiated dyspneic patients presenting to the Emergency Department (ED) can be challenging. In this scenario ordering biomarkers BNP or NT-proBNP as a binary test for diagnosing acute heart failure (AHF) may yield misleading results. Using prior study data we had retrospectively derived and validated a novel diagnostic prediction model for AHF simply utilizing clinician initial impression (percent likelihood of AHF), patient age and absolute NT-proBNP value.The present study prospectively assesses the diagnostic accuracy of the prediction model, as well as its effect on ED length of stay (LOS). Methods: The study is an international multicenter prospective controlled parallel randomized clinical trial of undifferentiated dyspneic patients presenting to the ED. After Emergency Physician (EP) assessment and chest xray (CXR) interpretation, dyspneic patients were considered for enrolment. Exclusion criteria were i) an obvious cause for dyspnea where the clinician would either treat for AHF without further investigation of another cause (pretest probability for $\mathrm{AHF}>80 \%$ ) or treat another cause and not investigate for AHF (pretest probability for AHF $<20 \%$ ), ii) Acute MI or iii) severe renal failure. Remaining patients (pretest probability for AHF 20-80\%) were randomized to either the blinded study arm with usual management or exposed study arm with management guided by the model result. Documentation of the time of EP initial assessment, time of EP patient disposition (either consultation or ED discharge) and at that point the EP final diagnosis were recorded. Using index ED and sixty day follow up records, the final diagnoses were adjudicated by two experienced clinicians blinded to the model result. For each arm, the proportion of agreed diagnoses were compared by a chi-squared test and the treatment effect was determined as an odds ratio with $95 \%$ confidence interval. LOS was measured in minutes and compared using a t-test. We assumed a control accuracy of $70 \%$ for diagnosing AHF; for an accuracy in the model group of $>92 \%$ we calculated a sample size of $75 /$ arm to have $80 \%$ power of detecting a significant difference. Results: With REB approval and informed consent, 150 dypnea patients undifferentiated for AHF were enrolled between November 2011 and October 2013.The mean age was 63 years with 37\% male; approximately half had a prior history of either AHF (49\%) or COPD (43\%). The Emergency Physician found signs of heart failure on $69 \%$ of CXRs, their pretest probability for AHF averaged $48 \%$ and $40 \%$ of their ED disposition diagnoses were AHF. Conclusion: The study will provide robust generalizable data for the utility of a novel clinical pre- diction model for diagnosing acute heart failure in the undifferentiated dyspneic ED patient.

Keywords: clinical decision making, congestive heart failure, cardiac biomarkers

\section{P106}

Emergency department diagnosis of pulmonary embolism: use of D-dimer, CT pulmonary angiography, and VQ scanning

B.J. Strauss, MD, N. Packer, BSc, M. Lipkus, BSc, S.L. McLeod, MSc, M. Klingel, MSc, A. Dukelow, MD, University of Western Ontario, London, ON

Introduction: Pulmonary embolism $(\mathrm{PE})$ is an elusive diagnosis in the emergency department (ED). Clinical decision rules (CDRs) that incorporate the PE Rule-out Criteria (PERC) and Wells' Score assist physicians in deciding the appropriate diagnostic method, but are inconsistently used by ED physicians. The objective of this study was to determine the number of d-dimer, CT pulmonary angiography (CTPA) and ventilation-perfusion (VQ) scans ordered in the ED for suspected $P E$ over a 1 year period and describe the diagnostic test characteristics prior to the implementation of a modified CDR for PE. Methods: This was a retrospective electronic medical record review of all diagnostic tests (d-dimers, CTPAs, and VQs) ordered by emergency physicians for suspected PE over a one-year period at an academic tertiary care centre (annual census 125,000). Results: There were 2722 patients investigated for potential PE. 2440 (89.6\%) patients had a d-dimer, 657 $(26.9 \%)$ were positive ( $\geq 240 \mathrm{~g} / \mathrm{L}), 1780(73.0 \%)$ were negative and 3 were unknown. Of those positive d-dimers, 291 (44.3\%) patients went on to have subsequent imaging. $366(55.7 \%)$ positive d-dimers had no subsequent imaging. Of those positive d-dimers that had subsequent imaging, $142(48.8 \%)$ patients had a CTPA, $25(17.6 \%)$ were positive for PE. 153 (52.6\%) patients had a VQ scan, 25 (16.3\%) were positive for PE. Of those negative d-dimers, $90(5.1 \%)$ patients went on to have imaging. $31(34.4 \%)$ patients had a CTPA, $2(6.5 \%)$ were positive for PE. $60(66.7 \%)$ patients had a VQ scan, $5(8.3 \%)$ were positive for PE. Of the 2722 patients included in the study, 282 (10.4\%) had imaging without a d-dimer. Of those, $168(59.6 \%)$ patients had a CTPA, 38 (22.6\%) were positive for PE. 114 (40.4\%) patients had a VQ scan, 13 $(11.4 \%)$ were positive for PE. There were 527 patients with negative imaging results. Of these, $5(1.0 \%)$ had additional imaging, all negative for PE. There were 28 patients with indeterminate imaging results. Of these, 15 (53.6\%) had additional imaging, all negative for PE. Conclusion: Adoption of a standardized emergency department clinical decision rule for the workup and diagnosis of PE may better risk stratify patients and reduce the number of laboratory and imaging tests ordered. Keywords: pulmonary embolism, D-dimer, imaging

\section{P107}

Use of a severe sepsis management bundle can improve ED care: preliminary findings from a quality initiative

S. Taylor, MD, M. McGowan, MHK, D. MacKinnon, MD, C. Hayes, MD MEd, L. Barratt, MSc, K. Gaunt, MSc, E. Nagatu, S.H. Gray, MD, University of Toronto, Toronto, ON

Introduction: Early recognition and treatment of sepsis reduces mortality. We assessed adherence to a collaborative ED-Critical Care model to identify septic patients early and promote timely antibiotics, fluids and lactate measurements. Methods: An inter-professional team developed a severe sepsis management bundle: i) triage flagging; ii) sepsis lab 
panel facilitated under a nursing medical directive; and iii) pre-printed order set to standardize ordering of antibiotics, fluids and serial lactates. A three-month (September-December 2013) retrospective review of ED patients admitted to ICU and Step-Up with pneumonia, sepsis or urosepsis was conducted after the bundle introduction. Clinical and administrative data was extracted with $10 \%$ independently reviewed for inter-rater reliability. Outcomes were time-to-treatments from triage. Results: 42 patients were identified: 1 had all elements of the bundle; 10 (24\%) had 2 elements (lab panel and order set); and the remainder were managed without the order set. Patients managed with at least 2 elements of the bundle had associated improvements (mean, SD): reduced time-to-first-antibiotics $(2: 24 \pm 2: 06$ vs $2: 46 \pm 2: 37 \mathrm{hr})$, receive at least 3L fluids ( $64 \%$ vs $52 \%$ ), reduced time-to- $3 \mathrm{~L}$ fluids $(3: 29 \pm 1: 32$ vs $4: 42 \pm 3: 12 \mathrm{hr}$ ), more lactates ordered ( $100 \%$ vs $74 \%)$, reduced time-tolactate $(1: 00 \pm 0: 57$ vs 1:17 $\pm 1: 09 \mathrm{hr})$, more serial lactates measured $(18 \%$

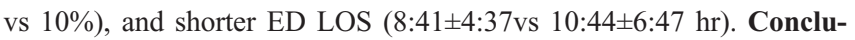
sion: A collaborative integrated sepsis care model in our ED has resulted in a trend to reduced time-to quality indices of sepsis management. Ongoing education and feedback to front line staff may further optimize care with uptake of the ED sepsis bundle.

Keywords: sepsis, quality improvement, resuscitation

P108

Online portfolios: the curriculum vitae 2.0

B. Thoma, MD, MA, T.M. Chan, MD, J.L. Sanders, MD, PhD, N. Joshi, MD, M. Lin, MD, Massachusetts General Hospital, Boston, MA

Innovation concept: As the job market for emergency physicians becomes saturated, Emergency Departments will look to hire candidates that meet their particular needs. The limitations of the traditional curriculum vitae (CV) make it difficult to differentiate between the multifaceted competencies of today's applicants. New online tools have made it feasible for physicians with no formal website development training to create impactful online portfolios. These portfolios may be used to (1) highlight the competencies of emergency medicine (EM) physicians and trainees, (2) demonstrate and model online professionalism, and (3) enhance collaboration and knowledge translation by making it easier to find work online. Methods: In 2013, a Wordpress.org website was created on the domain MedEdLIFE.org using the Avada theme by ThemeFusion. A template was uploaded for five participants: a medical student, an resident, fellow, junior attending, and senior attending in EM. Each was given a domain name (FirstLastName.MedEdLIFE.org) and coached to create portfolio posts. Posts could contain details of projects/publications in the form of text, images and videos along with hyperlinks to additional information and related projects/publications. The impact of publications could be quantified with links to Altmetrics and citation data. The entire site could be customized to individual preferences with optional pages available for personal/professional information, an educational philosophy and a personal blog. Contact information including links to social media profiles was provided to facilitate contact and collaboration. Curriculum, tool, or material: As of January 4, 201338 portfolio entries have been created. All portfolios are now discoverable using public search engines such as Google. The websites have been viewed 542 times despite minimal to no promotional efforts by the authors. Multiple personal inquiries have been made about the functionality and build of the portfolios by individual physicians and representatives of national medical organizations. Conclusion: This innovation demonstrates the ease of building a customized website to create an effective online academic portfolio. Highlighting the competencies of academically inclined emergency physicians could enhance one's competitiveness in the academic job market and lead to further opportunities for collaboration and scholarship while demonstrating a professional online presence.

Keywords: innovations in EM education, curriculum vitae, career

P109

The applicability of a clinical decision rule for chest radiography in patients with acute chest pain

S. Tran, MSc, O. Mall, MD, E. Hanel, MD, D. Lin, MD, McMaster University, Michael G. DeGroote School of Medicine, Hamilton, ON

Introduction: The need for Chest X-rays (CXRs) in all Emergency Department patients with acute chest pain has been questioned. A previous study derived a clinical decision rule showing that CXRs were not indicated for patients who have no history of congestive heart failure, were non-smokers and have normal lung examinations. The objective of this study was to retrospectively validate the rule in another setting. Methods: A retrospective chart review was conducted in two hospitals in Hamilton, Ontario between January and March 2012. Patients were included if they were 21 years or older and had a triage diagnosis of chest pain with one set of troponin results. Patients were excluded if they had evidence of a STEMI, blood pressure $<90 / 60$ or presented with acute cocaine use. We used a previously derived clinical decision rule to determine for all the included patients whether a CXR was indicated by the rule. The results of the rule was compared with the actual radiology report to determine whether the CXR actually changed management. Results: Two hundred and sixty charts were reviewed and 172 patients met the inclusion criteria. The rule found that 107 patients did not need CXRs and all these patients' CXRs did not have findings that required a change in medical management. The clinical decision rule showed $100 \%$ sensitivity (95\% CI 61-100\%) and a negative likelihood ratio of 0 (95\% CI $0-0.62)$. Conclusion: This clinical decision rule may be suitable for future prospective multicentre validation. This clinical decision rule is classified as a level IV with retrospective validation in more than one centre.

Keywords: emergency medicine, clinical decision rule, acute chest pain

\section{P110}

Characterizing how long-term-care patients use emergency department services in Regina, Saskatchewan

S. Trivedi, BSc, B. Little, BSc, E. Karreman, PhD, K. Lyster, BSc, MD, Regina Qu'Appelle Health Region, SK

Introduction: In light of recent local initiatives aimed at improving emergency department (ED) patient flow, we sought to characterize how residents of long term care (LTC) homes aged 65 and older utilize the services of the EDs in Regina, Saskatchewan and if the EDs were able to meet CAEP length of stay (LOS) benchmarks. Methods: A retrospective chart review was performed with a convenience sample of the first 50 patients who presented to the ED of both hospitals in Regina starting January 1, 2012. In order for the patients to be included they were required to be age 65 or older and to reside in one of the nine long term care homes in Regina, SK. We abstracted data from a variety of different clinical, demographic and administrative parameters. Results: The charts of 100 patients were reviewed (54 females; mean age: $82.6 \pm 9.0$ years old). Of the 100 visits, $27 \%$ were made by repeat 
users within the study window. The CTAS distribution for the LTC patients was found to be CTAS 1: 5\%, CTAS 2: 9\%, CTAS 3: $43 \%$, CTAS 4: $33 \%$ and CTAS 5: $10 \%$. The mean ED LOS for patients triaged as CTAS $1-3$ was $6.7 \pm 4.9$ hours, while for CTAS $4-5$ it was $4.4 \pm 2.9$ hours $(\mathrm{p}=.004 ; \mathrm{r}=.29)$. The mean ED LOS for admitted patients was $6.9 \pm 4.8$ hours. We found that $50 \%$ of patients were admitted, with $46 \%$ being discharged and $4 \%$ leaving without being seen. We also noted that $75 \%$ of patients were brought to the ED by EMS. Conclusion: Our findings suggest that the highest volume of acuity for the LTC patients falls within the CTAS 3 or 4 categories. Exactly half of our sample was admitted and a substantial amount of them required EMS transport. With respect to LOS, our results show that the two hospitals in Regina, SK are meeting the CAEP benchmarks for the 90th percentile in the CTAS 1-3, CTAS 4-5 patient groups and the median benchmark for the admitted patient group. It is our intent that the findings of this study will help guide future policy development.

Keywords: long-term care, patient flow, quality improvement

\section{P112}

\section{British Columbia Emergency Health Services' Aeromedical CBRNE/HazMat Response Program}

E.N. Vu, MD, DAvMed, R. Bernklau, H.E. Peet, R.S. Schlamp, R.T. Wand, BC Ambulance Service, BC

Introduction: Worldwide, there are more than 5 million known chemicals, with an additional 10,000-20,000 new chemicals developed each year. An estimated 500,000 shipments of hazardous materials occur daily throughout the US, resulting in thousands of annual exposures. Most commonly released substances are volatile organic compounds, herbicides, acids, and ammonia. Counter-terrorism response programs and industrial HazMat incidents have created the need for aeromedical EMS programs to have a plan in place to recognize these threats and respond safely. Regionalization of specialty resources and trauma centres capable of managing mass casualties, compounded by the potential for severe illness associated with CBRNE/HazMat incidents, underscores the necessity for EMS programs to dually provide early advanced care provider response, as well as a strategic operational plan for safe and expedient transport to definite care. Methods: We performed a review of the available medical and technical literature, training manuals, and published guidelines on CBRNE/HazMat response in the prehospital setting. Data and guidelines were reviewed to identify toxidromes and clinical conditions that met the following criteria, making them amenable to targeted prehospital intervention: 1) toxicant associated with acute life-or-limb threatening illness after exposure; 2) antidote or evidenced-based therapy identified for toxicant; 3 ) administration of antidote is time-sensitive in order to maximize treatment effect. Results: We identified 5 main CBRNE/HazMat scenarios that met the defined criteria above, and established antidote or evidencedbased treatment protocols to mitigate the immediate and sub-acute morbidity associated with these exposures. We then created standard operating procedures, recommended minimum training opportunities, and developed CBRNE/HazMat response kits for our aeromedical program. Our deployment program includes parallel screening and risk mitigation by CBRNE Technical Advisers, and is predicated on critical care paramedic engagement in the cold zone, after patient decontamination by HazMat technicians. Our aeromedical division response includes Level D PPE, standard operating procedures for aircraft stag- ing, patient decontamination criteria to prevent exposure and secondary contamination to non-isolated pilots and aircraft, as well as antidotes or treatment algorithms for the following toxicant exposures: 1) RSDL decontamination lotion for chemical and biological exposures; 2) auto-injectors and reconstitution algorithms for mega-dose and/or mass-casualty antidote administration for chemical warfare agents, nerve agents and organophosphates; 3 ) antidote and treatment algorithm for confirmed or suspected cyanide toxicity; 4) antidote for hydrofluoric acid/fluoride exposures; and 5) symptom management options for pulmonary irritants/asphyxiants. Conclusion: Successful CBRNE/HazMat response includes appropriate recognition, response, and recovery. Proper risk stratification and prompt response with prehospital antidote administration can prevent secondary life loss and minimize patient morbidity and mortality. Our aeromedical program has operationalized parallel processes with our CBRNE Technical Adviser program to promptly, safely, and effectively deliver out-ofhospital critical care and aeromedical evacuation to patients exposed to intentional or accidental industrial or toxicant exposures.

Keywords: CBRNE, HazMat, antidote

\section{P113}

Emergency supply of blood products for aeromedical evacuation British Columbia Emergency Health Service's (BCEHS) pilot program

K. Polson, S. Feenstra, H. Mah, R.T. Wand, K. Chipperfield, MD, E.N. $\mathrm{Vu}, \mathrm{MD}, \mathrm{DAvMed}, \mathrm{BC}$ Ambulance Service, BC

Introduction: Recent paradigm shifts in choice of resuscitation fluids in both trauma and medical patients has resulted in earlier and more aggressive use of blood products in select cases. Significant operational challenges exist when requests are made to move a patient to definitive care with ongoing or anticipated transfusion requirements from rural sites with limited or no blood supply. British Columbia (BC) Critical Care Paramedics (CCP) have been transfusing blood products during aeromedical evacuation (AME) for decades; however, historically they have had to use what is available at the referring site. In some circumstances, transport delays are incurred waiting for blood products to be made available to CCP crews for transport. In many circumstances, no blood products are available at all at the referring site. Methods: Following a consultative process with Trauma Services BC, the BC Provincial Transfusion Medical Advisory Group, and in collaboration with Vancouver Coastal Health Authority's Transfusion Medicine Services (VCHA-TMS), we identified system factors that could be adapted to improve system efficiencies across all stakeholder groups. The goal was to create an integrated system resulting in more timely access to a dedicated store of blood products for CCPs during AME, and improved provincial use of blood components in compliance with Provincial and National transfusion standards. Results: Goal-directed resuscitation with blood products in select trauma and medical patients has gained traction over the past several years. This has translated to an increased demand for mobile critical care services to administer blood products in all phases of the out-of-hospital care continuum. In order to align patient care delivery with current trauma and medical resuscitation paradigms, BCEHS Critical Care Operations has developed a parallel dispatch process with VCHA-TMS to allow CCPs 24-hr access to a dedicated store of blood products within 10 minutes from dispatch, in a Federally approved transport container. CCP training and standard operation procedures were updated to meet Provincial and Federal 
Regulatory standards. Previous serial processes between EMS dispatch, air ambulance operations, and regional blood banks have been replaced with streamlined parallel processes resulting in quicker access to blood products, more rapid response to patient-side, translating to more rapid goal-directed resuscitation and transport to definitive care. Conclusion: Early resuscitation with component or whole blood products is quickly emerging as best practice in select trauma and medical patients. EMS agencies with mobile critical care services need to adapt their dispatch protocols and treatment guidelines in order to align themselves with current practice and recommendations. We describe the protocols, checklists and integrated standard operating procedures of a collaborative process resulting in streamlined availability and dispatch of blood products in order to optimize system response to patient-side, to improve blood product resource utilization across various regions, and to improve patient care delivery models.

Keywords: blood products, transfusion, aeromedical evacuation

\section{P114}

The desire for a core emergency medicine clerkship rotation at Memorial University

Z. Warren, L. Taylor, BSc, T. Renouf, MD, Memorial University, St. John's, NF

Introduction: A majority of medical schools in Canada require completion of a core rotation in emergency medicine (EM) during clerkship. There is currently a need for emergency department physicians across Canada. A recent study has linked mandatory rotations in EM with application to post-graduate programs in EM. Memorial University currently does not mandate completion of such a rotation. The purpose of this study is to determine opinions of medical students regarding implementation of a mandatory core EM rotation during clerkship. Methods: A brief survey was designed using Survey Monkey to distributed to all current medical students at Memorial University. Results: The response rate was $45 \%$ (118/260) with a well-distributed response from both pre-clerkship and clerkship students. $77 \%$ of students feel that they do not get enough exposure to emergency medicine and $83 \%$ feel that there should be a mandatory rotation in the specialty. $23 \%$ of students currently see emergency medicine as a likely career choice. Opinions were split regarding the length of a proposed rotation with $59 \%$ favouring a 4 -week rotation vs. $38 \%$ favouring a shorter, 2-week experience. Participants chose ECG interpretation, airway management, $\mathrm{x}$-ray interpretation, suturing, and venous access as skills they would most like to become proficient in during the rotation ( $93 \%$ vs. $88 \%$ vs. $87 \%$ vs. $74 \%$ vs. $67 \%)$. The top five patient presentations desired were chest pain, trauma, altered mental status, abdominal pain, and shortness of breath ( $84 \%$ vs. $80 \%$ vs. $61 \%$ vs. $61 \%$ vs. $57 \%$ ). $84 \%$ of participants think paramedic ride-outs should be part of a proposed EM rotation, with $32 \%$ believing it should be mandatory. Conclusion: There is significant interest in the implementation a mandatory core emergency medicine rotation at Memorial University.

Keywords: emergency medicine, education, clerkship

\section{P115}

Caregiver satisfaction with children's pain management in the emergency department

L. Weingarten, MD, J. Kircher, MD, A. Newton, Ph.D, K. Dong, MD, MSc, R.J. Rosychuk, PhD, S. Curtis, MD, MSc, S. Ali, MDCM, University of Alberta, Faculty of Medicine and Dentistry, Edmonton, AB
Introduction: Pain is a common reason for seeking care in the emergency department (ED) and family members are generally considered the most appropriate proxy to interpret young children's pain. A better understanding of the family's perspective is key to improving children's ED pain management. This project surveyed children's caregivers to examine their satisfaction with pain management in two EDs. Methods: Using a prospective, cross-sectional design, we recruited a convenience sample of ninety-seven caregivers. Trained research assistants distributed a self-report survey to measure caregiver satisfaction with their child's pain management at one pediatric and one general ED. The survey collected data on parent/child demography, caregiver perspectives on analgesia and overall satisfaction with ED pain management. Data are presented using descriptive statistics. Results: Ninety-seven families participated (general $E D, n=46$; pediatric $E D$, $\mathrm{n}=51)$. Most caregivers were mothers $(71 \%)$ and older than 31 years (81\%). The mean age of children was 9.2 years (SD 5.2) and $52(53 \%)$ were boys. Common presenting issues included musculoskeletal pain $(42 \%)$, headache $(17 \%)$ and abdominal pain $(7 \%)$. The mean caregiver-reported maximum pain score was $75 \mathrm{~mm}$ on a $100 \mathrm{~mm}$ VAS scale $(95 \%$ CI: 72,80$)$. The mean pain score during survey administration was $39 \mathrm{~mm}$ (95\% CI: 33, 44). Eighty caregivers $(90 \%)$ were satisfied with their children's pain management and nine $(10 \%)$ were dissatisfied. A minority of caregivers (43\%) agreed they would like a stronger dose of pain medication if their child were still having pain. Inter-site analyses are currently underway, and will be available for presentation. Conclusion: Caregivers reported that their children have significant pain during an ED visit. Although many children experienced severe pain, most caregivers reported satisfaction with ED pain management. The relationship between caregiver satisfaction and pain management should be explored further, as satisfaction does not necessarily correlate with appropriate analgesia.

Keywords: pain management, pediatrics, satisfaction

\section{P116}

Prevalence of hazardous drinking varies between socioeconomic groups in an urban academic emergency department

I. Whatley, BSc, F. Ilyas, BHSc, M. Klaiman, MD, L. Thurger, MD, MSc, University of Toronto, Toronto, ON

Introduction: Alcohol dependence is estimated to be $2.6 \%$ in the Canadian population. However, the prevalence of hazardous drinking and its distribution across different socioeconomic groups remains unclear. Hazardous drinking is characterized by drinking that leads to harmful consequences to the participant, without that participant meeting all the requirements for alcohol dependence. Establishing the prevalence of hazardous drinking is of interest as alcohol has been implicated in a variety of health conditions and is a major cause of emergency department (ED) visits. Numerous validated screening tools have been developed to detect alcohol problems ranging from at-risk drinking to alcohol dependence. These tools help to identify patients that may benefit from brief interventions in the ED. Unfortunately, alcohol screening in the ED is rarely a priority and opportunities for intervention are lost. This study aims to establish the prevalence of alcohol problems in an urban, academic emergency department and determine differences between socioeconomic groups. Methods: Prevalence of hazardous drinking in the Sunnybrook Health Sciences Centre Emergency Department was determined by screening patients using the Fast Alcohol Screening Tool (FAST) questionnaire. In addition, demographic data was collected including age, sex and socioeco- 
nomic status. Results: The prevalence of hazardous drinking in the ED was $12 \%$. Hazardous drinking was highest in the $\$ 25,000-50,000$ (24\%) income group, and second highest in the $\$ 50,000-100,000$ group (14\%), while the $<\$ 25,000$ group had a lower prevalence (4\%) and the $>\$ 100,000$ group had a prevalence of $0 \%$. Conclusion: The results of this study show a high prevalence of hazardous drinking in the emergency department population. Furthermore, there appears to be a correlation between hazardous drinking and socioeconomic status. Further research is warranted to evaluate the role socioeconomic status may or may not play as a risk factor for hazardous drinking. The results of this study can be used for ongoing knowledge translation projects to improve the quality of care for individuals with hazardous drinking behaviours to ensure they are properly identified and cared for.

Keywords: hazardous drinking, emergency department, socio-economic status

\section{P117}

\section{Flipping the clerkship classroom}

S.H. Yiu, MD, University of Ottawa, Department of Emergency Medicine, Ottawa, ON

Innovation concept: The flipped classroom is used increasingly in education. In this model, learning is self-paced and lessons are interactive and mastery-based. Multiple flipped classroom methods have been suggested in the education literature, but no specific flipped Emergency Medicine clerkship curriculum has been published. Educational Objectives: Flipping our clerkship core tutorials to allow for higherorder classroom discussions. Methods: Topics from the Emergency Medicine Clerkship curriculum selected by the Clerkship Directors of Emergency Medicine act as the blueprint. Each topic is broken down into short videos lasting 5-15 minutes adhering to multimedia principles. All videos were hosted on a public website. Before class, students watch videos with specific learning objectives and are urged to take notes. During class, students are divided into small groups and are presented with clinical cases. As a group, they determine decisions regarding clinical management for each case. The tutors intervene when the students have questions. The videos remain accessible to students throughout their Emergency Medicine rotation and beyond. Curriculum, tool, or material: Class time is used for discussion about clinical decisions rather than core knowledge transfer. Students engage in discussions and identify muddy points quickly. Tutors intervene to clarify only when needed. Students often raise questions not brought up in previous lecture-based tutorials. Students feel they have delved deeper into management rather than reciting facts, and feel 'this is a better way to learn'. Tutors roles have changed from lecturer into facilitator and prepare differently. Students also return to videos as resources for exam studying and clerkship preparation. Conclusion: Future Directions: Our next step is to provide online exercises for students before class as a model of deliberate practice. Research measuring knowledge retention and application is under way. How it enhances EM education: Videos standardize core content students receive. Students study them on their own pace. Valuable face time with the tutor is not used to deliver core materials. It is used to engage students in interactive discussion to apply knowledge into practical, nuanced clinical cases and higher order learning.

Keywords: innovations in EM education, emergency medicine, clerkship

\section{P118}

Are faster doctors better teachers? A comparison of teaching versus productivity in the emergency department

O. Yusuf, MD, M. Gatien, MD, A. Bishnoi, MD, J.R. Frank, MD, University of Ottawa, Department of Emergency Medicine, Ottawa, ON

Introduction: Clinical education is a priority in medicine that occurs in parallel with patient care. Clinical supervisors may feel that the educational objectives of learners conflict with clinical productivity, and that a trade-off exists between the two. Despite the importance of balancing teaching with productivity in the emergency department (ED) setting, there is a paucity of literature on the subject. The aim of this study was to clarify the relationship between teaching and productivity. Methods: We conducted a retrospective database review of two academic emergency departments from July 2010 to May 2013 comparing the productivity and teaching score of staff physicians. Productivity was measured by patients seen per hour in the ambulatory care sections of the department, and teaching was measured by the overall score staff received from residents using a likert scale on our evaluation software (One45). We measured the relationship between productivity and teaching using a Pearson correlation. We also conducted a survey to compare results with resident perception of teaching and productivity. Results: Using 1141 shifts and 4737 evaluations of staff we found a significant positive correlation between productivity and teaching scores (correlation 0.304, p-value 0.036). Our survey demonstrated diverse perceptions among residents regarding the perceived relationship between teaching and productivity. Conclusion: This study counters the notion that to optimize teaching of learners on shift, clinical productivity needs to be sacrificed. It is the first known Canadian study on this topic, and the largest of its kind in the emergency medicine setting. Professional development for physicians must emphasize teaching techniques that address the perceived tension between productivity and education.

Keywords: productivity, teaching, education 